\title{
Electrical characterization of conductive ion tracks in tetrahedral amorphous carbon with copper impurities
}

\author{
Dissertation \\ zur Erlangung des mathematisch-naturwissenschaftlichen Doktorgrades \\ „Doctor rerum naturalium“ \\ der Georg-August-Universität Göttingen \\ im Promotionsprogramm Prophys \\ der Georg-August-University School of Science (GAUSS)
}

vorgelegt von

Hans-Gregor Gehrke

aus Merseburg

Göttingen, 2013 
$\underline{\text { Betreuungsausschuss }}$

Prof. Dr. Hans Hofsäss, II. Physikalisches Institut, Universität Göttingen

Dr. Ulrich Vetter, II. Physikalisches Institut/AG Prof. Hofsäss, Universität Göttingen

Mitglieder der Prüfungskommission

Referent: Prof. Dr. Hans Hofsäss, II. Physikalisches Institut, Universität Göttingen

Korreferent: Prof. Dr. Christian Jooß, Materialphysikalisches Institut, Universität Göttingen

Weitere Mitglieder der Prüfungskommission:

PD Dr. Jörn Grosse-Knetter, II. Physikalisches Institut/AG Prof. Quadt, Universität Göttingen Prof. Dr. Hans-Ulrich Krebs, Materialphysikalisches Institut, Universität Göttingen

Prof. Dr. Markus Münzenberg, I. Physikalisches Institut, Universität Göttingen

Prof. Dr. Michael Seibt, IV. Physikalisches Institut, Universität Göttingen

Tag der mündlichen Prüfung: 


\section{Contents}

1 Introduction 5

1.1 Motivation . . . . . . . . . . . . . . . . . . 5

1.2 Objectives of this thesis $\ldots \ldots \ldots \ldots \ldots$

$\begin{array}{lll}2 & \text { Fundamentals } & 9\end{array}$

2.1 Tetrahedral amorphous carbon. . . . . . . . . . . . . . . . . . . 9 9

2.2 Growth model and amorphous carbon deposition . . . . . . . . . . 11

2.3 Stopping of ions and track formation $\ldots \ldots \ldots \ldots \ldots$

2.3 .1 Stopping of ions . . . . . . . . . . . . . . . 11

2.3 .2 Nuclear stopping power. . . . . . . . . . . . . . . . . . . 12

2.3 .3 Electronic stopping power $\ldots \ldots \ldots \ldots$

$2.3 .4 \quad$ Charge state. . . . . . . . . . . . . . . . . . . . . 14

$2.3 .5 \quad$ Simulation of stopping power and charge states $\ldots \ldots \ldots . \quad 15$

2.3 .6 Track formation . . . . . . . . . . . . . . . . . . . . . 18

2.3 .7 Track formation in ta-C $\ldots \ldots \ldots \ldots \ldots \ldots$

2.3 .8 Conductive tracks in other materials . . . . . . . . . . 23

2.4 Electron transport mechanisms $\ldots \ldots \ldots \ldots \ldots \ldots \ldots$

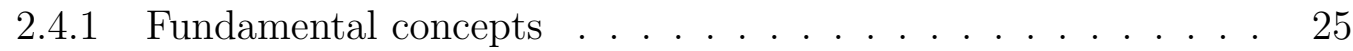

2.4 .2 Transport of electrons $\ldots \ldots \ldots \ldots \ldots$

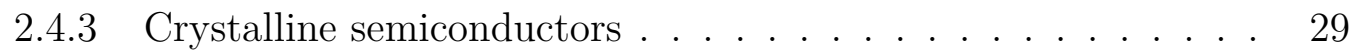

$2.4 .4 \quad$ Asymmetric conduction - Schottky contact. . . . . . . . . . 30

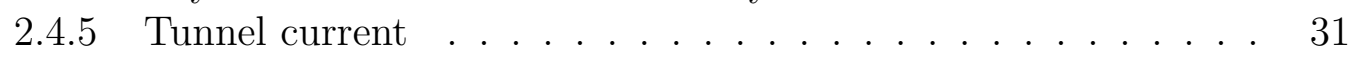

2.5 Conductivity in amorphous materials $\ldots \ldots \ldots \ldots \ldots$

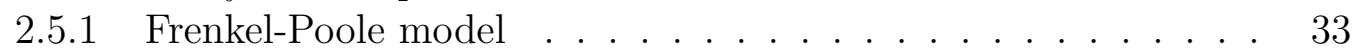

$2.5 .2 \quad$ Variable Range Hopping . . . . . . . . . . . . . . . . . . . . 36

$2.5 .3 \quad$ Conductivity of ta-C $\ldots \ldots \ldots \ldots$

2.5 .4 Impurity conduction in ta-C . . . . . . . . . . . . . . . 42

2.5 .5 Track conductivity in ta-C $\ldots \ldots \ldots \ldots$. . . . . . . 43

3 Experimental methods 45

3.1 Mass selected ion beam deposition $\ldots \ldots \ldots \ldots \ldots$

3.1.1 ta-C fabrication . . . . . . . . . . . . . . . . . . . . . . . 46

3.2 Swift Heavy Ion Irradiation $\ldots \ldots \ldots \ldots$

3.3 Transmission electron microscopy - TEM . . . . . . . . . . . . . . 48

$3.3 .1 \quad$ Sample preparation $\ldots \ldots \ldots \ldots \ldots \ldots \ldots$

3.3 .2 TEM-imaging $\ldots \ldots \ldots \ldots \ldots \ldots$ 
3.4 X-ray photoemission spectroscopy - XPS . . . . . . . . . . . . . . . . 49

3.5 Electrical characterization . . . . . . . . . . . . . . . . . . 51

3.5 .1 Carriers ............................ 54

3.5 .2 Sample back-contacts . . . . . . . . . . . . . . . . . 54

3.5 .3 Wedge bonding . . . . . . . . . . . . . . . . . . . . . 54

3.6 Atomic force microscopy - AFM . . . . . . . . . . . . . 56

4 Structural characterization by XPS 59

5 Macroscopic electrical characterization of ta-C conductivity 63

5.1 Unirradiated virgin ta-C . . . . . . . . . . . . . . 63

5.2 Irradiation of ta-C . . . . . . . . . . . . . . . . . . . 65

5.3 Copper impurities in ta-C . . . . . . . . . . . . . . . . . . 68

5.4 Comparison of $I$ - $V$-characteristics of ta-C: $\mathrm{Cu} \ldots \ldots \ldots$. . . . . . . . 74

5.5 Frenkel-Poole model for virgin ta-C . . . . . . . . . . . . . . . . . 75

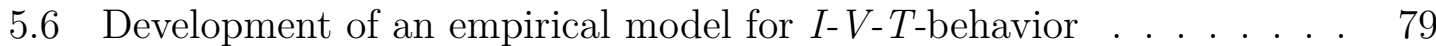

5.6 .1 Discussion of temperature dependence of zero-bias conductivity 81

5.6 .2 Discussion of curvature of the $I$ - $V$-characteristics $\ldots . . . .991$

5.7 Summary of the $I$ - $V$-T-model $\ldots \ldots \ldots \ldots$. . . . . . . . . . . . 95

5.8 Comparison of track and matrix conductivity . . . . . . . . . . . . . 97

5.9 Scalability of ensemble measurements . . . . . . . . . . . . . . . . 100

5.10 Residual Conductivity and stability of films . . . . . . . . . . . . . . 102

5.11 Summary - contact pad characterization . . . . . . . . . . . . . 107

6 Microscopic characterization of ta-C by AFM 109

6.1 Tracks and hillock . . . . . . . . . . . . . . . . . . . . . . . 109

6.2 Effect of copper . . . . . . . . . . . . . . . . . . . . . . . . . . 112

6.3 Effect of charge state selection . . . . . . . . . . . . . . . . . . 120

6.4 Comparison of temperature-dependent AFM and pad conductivities . 125

6.5 Summary AFM measurements . . . . . . . . . . . . . . . . . . 128

\begin{tabular}{lll}
\hline 7 & Characterization of multilayer structures & $\mathbf{1 3 1}$
\end{tabular}

7.1 TEM characterization . . . . . . . . . . . . . . . . . 133

7.2 Macroscopic electrical characterization . . . . . . . . . . . . . 135

7.3 AFM measurements . . . . . . . . . . . . . . . . . . . . . . . . . . . 139

7.4 Summary multilayers . . . . . . . . . . . . . . . . . . . . . . . . . . . . 142

$\begin{array}{lll}8 & \text { Summary } & 143\end{array}$

8.1 Conclusions . . . . . . . . . . . . . . . . . . . . . . . . . . 143

8.2 Outlook . . . . . . . . . . . . . . . . . . . . . . . . 146

\begin{tabular}{ll}
\hline Bibliography & 151
\end{tabular} 


\section{Introduction}

\subsection{Motivation}

Collisions of atomic or sub-atomic particles are a fundamental field of physical and technological research. Most prominent, the frontier of high energy physics is based on collisions of super-fast particles in collider experiments, pursuing, for example, the search of the Higgs boson [1]. In nuclear physics, new heavy elements are found in accelerator experiments as well. Element 112 was discovered by colliding Zn onto $\mathrm{Pb}$ targets at GSI [2]. The heaviest element confirmed up to date has the atomic number $118[3$ and was created analogously. Historically, the discovery of nuclear fission sparkled the research of nuclear reactions induced by collisions. The development of reactors, built to investigate nuclear fission, was the first strong artificial source of high-energy irradiation. Besides nuclear reactions, modifications of irradiated bulk materials were discovered opening a new field of research on radiation damage caused by swift heavy atomic and sub-atomic projectiles. The microscopic nature of this damage was first described by Young et al. in 1958 [4]. They were able to show the material modification, caused by each individual impinging particle, by etching the irradiated surface of LiF crystals. The modification along the trajectory would increase the etching rate and small craters for each impact are formed on the crystal surface. They also proposed the first concept of defect production by the stopping process along the projectile path. One year later, Silk et al. published direct proof of this damage showing individual tracks in mica with the aid of transmission electron microscopy [5]. In addition, natural tracks caused by cosmic irradiation gained interest. The moon rocks, obtained during the Apollo missions [6], and geologic specimens in general are dated and analyzed by using tracks caused by particle irradiation 7,8 .

The development and availability of controlled ion accelerators beams have allowed more systematic analysis of the stopping processes by the creation of different synthetic ion tracks. For example, Trautmann et al. gained detailed information on the track formation in LiF by using well controlled ion beams instead of fission products for the radiation [9]. Since the discovery of first fission tracks 50 years ago, a multitude of research and application around swift heavy ion tracks has developed 10,11. Generally, the irradiation with swift heavy ions will cause material modifications within the track volume, which are hard to obtain otherwise. The high energy deposition within very short time frames results in enormous temperature spikes followed by quenching leading to the freezing of non-equilibrium states. In addition, technical applications take advantage of the nano-sized track dimensions as well as the large 
areas that can be irradiated in parallel. The material transformations provide a vast field of fundamental research to study non-equilibrium physics. A prominent example for technical use is the track formation in polymers. Here, the stopping of swift heavy ions produces trails of damage changing the chemical reaction rate within the track volume, allowing selective etching and pore formation in polymer materials $[12$. The resulting nanometer- to micrometer-sized pores with high aspect ratios are the basis for different applications. A process, called track ion lithography, uses the pores as a mask. Filled pores create high aspect metallic nanowires [13]. These wires can be applied as field emission structure [14]. The pores may also be used as a mask to modify materials underneath by etch processes. Bernhardt et al. developed field emission structures implementing ion track lithography to create their devices [15]. Further applications of ion tracks in micro- and nano-electronics were proposed by Fink et al. and Hoppe et al. 16, 17]. A field of fundamental research and technical applications is the track formation in magnetic- and superconductive materials. In case of superconductors, the formation of straight defect rich cylinders may be ideal for pinning magnetic flux lines 18 . For example, the magnetic behavior of hexaferrites can be modified by swift heavy ion tracks 19. Furthermore, ion irradiation may plastically deform targets (ion hammering) providing information on the mechanical behavior 20]. The modifications at impact points of ions are an interesting tool of surface physics; a review of this field is provided, for example, by Aumayr et al. [21].

Among the multitude of different types of material modifications, induced by ion track formation, a relatively unique kind is found in amorphous carbon (a-C). Here, the energy deposition of swift heavy ions form highly conductive ion tracks by graphitizing the path of the projectile. This phenomena was first reported by Waiblinger et al. over a decade ago. Since then, research has been done in order to understand the formation and improve the conductivity of these embedded filaments [22 28]. In addition, some applications for conductive ion tracks have already been suggested and explored. Schwen et al. investigated the field emission capabilities of embedded conductive ion tracks [29]. Gehrke et al. suggested a more elaborated device [30] combining latent tracks in polymer producing lithographic electrode structures in connection with conductive tracks in amorphous carbon. This technique exploits another advantage of swift heavy ions. The same projectile produces the track in the entire film sandwich aligning the structures to nanometer-precision without any effort.

Without an understanding of the dominant conduction mechanisms and knowledge about factors affecting the transport, an application of conductive ion tracks in devices is problematic. The characterization is achieved by one of two approaches for electrical characterization, either large ensembles of tracks that are analyzed collectively using macroscopic contacts, or individual tracks are measured microscopically using atomic force microscopy (AFM). The identification of conduction mechanisms requires temperature dependent characterization. This method has been applied recently on individual tracks by AFM [24,31]. Temperature dependent pad data has 
been available for some time, but a direct comparison of AFM- and pad-data has been difficult 25, 32, 33. A major problem is the reproducibility of amorphous carbon films exhibiting the same electric behavior. Slight differences in the hybridization ratio cause significant differences in conductivities [26]. Finally, films irradiated by typical fluences exhibit a low resistivity, requiring careful contacting to produce reliable data with little contact resistance. A correct description of the electric behavior is only possible when these factors are controlled.

Another aspect of amorphous carbon has been the search for suitable doping to create semiconductor devices. So far, neither p- nor n-type doping has been successfully achieved [34,35]. According to theoretical descriptions, doping will not be possible in an effective manner due to the structural and chemical properties of a-C (chapter 2.5.4). Nonetheless, the incorporation of impurities in a-C affects the conduction behavior. Reducing and tuning the resistivity of tracks would be useful for device applications. In addition to a high conductivity contrast of tracks and matrix, reproducible and homogeneous tracks are important for applications. Microscopic analysis by AFM has revealed a large distribution of track conductivities under typical irradiation conditions (e.g. ref. [24]). Typical ion irradiation conditions apply stripper foils directly before the target causing an irradiation with differently charged ions according to the equilibrium charge distribution. The direct effect of different charge states is part of this work.

With regard to possible applications, a large conductivity contrast of matrix and track is desired besides highly conductive tracks. This ratio can be manipulated by the irradiation conditions and the properties of the pristine carbon films. Zollontz et al. [26] investigated the effect of the hybridization ratio of the carbon. Nix [25] and Krauser et al. 31, 36] measured the effect of various impurities on track conductivity. Copper has been the most promising additive in terms of improving track conductivity while preserving the matrix resistivity. 


\subsection{Objectives of this thesis}

The main objective of this thesis is the comprehensive analysis of the electric behavior of tetrahedral amorphous carbon, particularly with regard to the effects of swift heavy ion irradiation and copper impurities. Several carbon films with different copper concentration were synthesized and underwent structural analysis by x-ray photon spectroscopy (chapter 4). The import hybridization ratio of the carbon matrix is measured under the the effect of copper. In addition, all samples underwent electrical characterization using macroscopic contact pads at temperatures ranging from $20 \mathrm{~K}$ to $380 \mathrm{~K}$ (chapter 5). In this mode, millions of tracks are measured in parallel by each pad contacted. The resulting currents are normalized to specific conductivities of tracks and matrices in order to be able to make direct comparisons. Furthermore, a model-description in terms of simple conduction mechanisms is presented and fitted to the data (chapter 5.6). The results allow an interpretation of effects caused by copper impurities and tracks on the conductivity. The data is also analyzed to find the best doping concentration to create the highest conductivity enhancement in tracks (chapter 5.8). Microscopic analysis of conductive ion tracks was conducted by AFM (chapter 6). The statistical distributions of tracks in carbon films with different copper content are compared in order to better understand the track formation. The direct effect of the initial charge state on track formation was observed by comparing different charge states of the impinging ions. The normalized average track conductivities obtained by AFM are compared to the results of the macroscopic measurements. This comparison shows the degree of integrity of both experimental approaches showing possible differences and sources of errors. Finally, a device concept to create interrupted ion tracks is presented (chapter 7). The electric transport is measured by AFM and contact pad characterizations. The structure is also investigated using cross section transmission electron microscopy. The comparison of all analytical techniques provides a good understanding of the electric transport as well as ideas for improving the device. 


\section{Fundamentals}

In this section the fundamentals and the theoretical background of the physical phenomena studied in this work are presented. In the last decades, a multitude of research has been performed with regard to amorphous carbon providing a vast amount of publications. A collection of knowledge of amorphous carbon was published by Robertson [37] and Silva [38].

\subsection{Tetrahedral amorphous carbon}

Carbon, the sixth element of the period table, has many unique properties. Most famous are the superlatives of diamond, which is the hardest crystalline structure (largest bulk modulus), has the highest thermal conductivity, a high optical refraction index, and is a wide band gap semiconductor with gap of $5.5 \mathrm{eV}$ [39, 40]. Simultaneously, carbon forms graphite, an anisotropic metal [41]. Furthermore, modern research has shown interesting nano-configurations of carbon such as carbon nanotubes [42], fullerenes [41, 43 and graphene [44]. The key to the very different physical properties of carbon is its electron bond structure.

Carbon forms different bond structures, graphite and diamond are the bulk crystalline allotropes, where graphite is the thermodynamically stable configuration at normal conditions. The hexagonal layered structure results from $\mathrm{sp}^{2}$ hybridized carbon atoms forming strongly-bonded layers. The layers, on the other hand, are only bonded weekly by Van der Waals forces. This results in high anisotropies of physical properties such as electric and thermal conductivity or the speed of sound [45, 46]. Graphite has no band gap and exhibits a metallic conductivity. Electronically, the reason for this behavior is again the $\mathrm{sp}^{2}$ hybridization. Two of the three $\mathrm{p}$ orbital electrons form a strong $\sigma$ bond in the hexagonal planes, while the last $\mathrm{p}$ electron forms a week $\pi$ bond normal to the plane. The diamond structure, on the other hand, is the thermodynamically stable form of carbon at high pressures and temperatures, which is metastable at normal conditions. The lattice structure is an isometric-hexoctahedral cubic structure formed by $\mathrm{sp}^{3}$-hybridized carbon. Here, all electrons of the outer s and p orbitals form strong $\sigma$ bonds to the neighboring atoms, leading to the very stable nature of diamond, its high electrical resistivity and its optical transparency. The nanostructures of carbon are formed of two-dimensional sheets of the $\mathrm{sp}^{2}$ bond structure of carbon exhibiting properties of two-dimensional crystals leading to extraordinary properties such as extreme hardness, high current densities and electron mobility 41,43$]$. 
Finally, amorphous structures containing mixtures of $\mathrm{sp}^{2}$ and $\mathrm{sp}^{3}$ hybridization are possible. The ratio of the two hybridization types of carbon influence the properties of the amorphous carbon (a-C). The electrical conductivity, band gap, density, hardness, and transparency are dependent on this ratio. It is possible to tailor materials with properties to desired specification. As the $\mathrm{sp}^{2}$ bond ratio is reduced the behavior tends towards diamond properties. The conductivity decreases dramatically, density and hardness increase and the band gap widens [37.

Another factor, besides the hybridization ratio is the hydrogen content of the a-C structures. Hydrogen is easily integrated by saturating $\mathrm{sp}^{2}$ bonds forming $\mathrm{sp}^{3}$ bonds. It can be added on purpose, but often, it is unavoidably incorporated because of the gases used for the synthesis process. For many mechanical properties, such as hardness and roughness of films, the H-content is not too important. However, the electrical behavior is significantly influenced by hydrogen even at low concentrations.

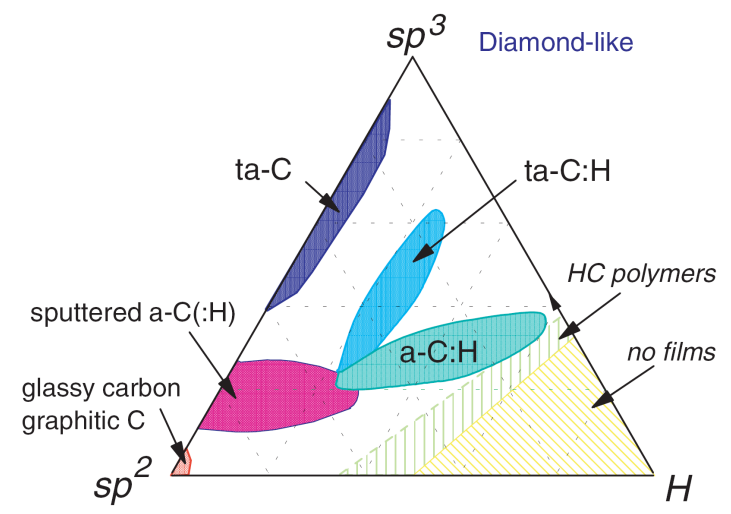

Figure 2.1: Ternary phase diagram illustrating the different types of amorphous carbon in a triangular diagram taken from ref. [37. The interesting ta-C phases of the work are found on the left side towards high $\mathrm{sp}^{3}$ hybridization.

In general, the possible configurations can be shown in a triangular plot, a ternary phase diagram (figure 2.1), where one corner represents pure graphite, the next pure diamond and the last pure hydrogen. Regions in the center of this triangle are called amorphous carbon (a-C) or a-C:H if a significant amount of hydrogen is incorporated. Amorphous carbons with high $\mathrm{sp}^{3}$ hybridization ratios are called tetrahedral amorphous carbon (ta-C). In case of a significant hydrogen content the material is called ta-C:H. In this work ta-C films with low hydrogen and high $\mathrm{sp}^{3}$ content are of interest situated along the left side of the triangle in figure 2.1. A synonym to amorphous carbon in general is diamond-like carbon (DLC). Technical details on obtaining hydrogen-free samples in the scope of this work are discussed in chapter 3.1. Furthermore, the electron transport phenomena in ta-C are discussed in chapter 2.4. An elaborated and extensive overview on many aspects of DLC is provided by Robertson [37] and Silva 38]. 


\subsection{Growth model and amorphous carbon deposition}

The formation of DLC in general and ta-C in particular can only be achieved by depositing the carbon with sufficient energy and allow for quick relaxation (nonequilibrium deposition). A prominent method, also used in this work, is ion deposition, where ions are accelerated onto the target with the desired energy. Other deposition methods include sputtering, pulsed laser deposition (PLD), (magnetron) sputtering, and cathode vacuum arc deposition. An overview and a collection of references discussing advantages of each method is given by Robertson [37]. For ta-C, all methods rely on depositing the carbon atoms or ions with energies in the range of $20 \mathrm{eV}$ to $1000 \mathrm{eV}$. In case of ion beam synthesis, the energy can easily be controlled to high precision independently of other parameters, such as flux and hydrogen content. Other methods as sputtering or PLD lead to larger deposition rates, which are more economical in a manufacturing environment. The technical details of the ion beam deposition used in this work are found in chapter 3.1.

The $\mathrm{sp}^{3}-\mathrm{sp}^{2}$ ratio of ta- $\mathrm{C}$ depends on the deposition energy. While the complete growth mechanism is not entirely understood, it is commonly accepted that the formation of the $\mathrm{sp}^{3}$-rich matrix is formed underneath the surface. This process is called subplantation [47. The carbon ions with $20 \mathrm{eV}$ to $200 \mathrm{eV}$ have a range of $0.5 \mathrm{~nm}$ to $1.5 \mathrm{~nm}$ according to SRIM [48]. An in-depth study of ion range during a-C deposition has been performed by Neumaier et al. [49]. The energy is mainly lost by nuclear stopping (chapter 2.3.2). The energy is transferred to other atoms (displacements and sputtering) and couples via phonons to the lattice. The pressure exerted by this subplantation prefers the denser $\mathrm{sp}^{3}$ configuration of the carbon. Hofsäss describes this idea in the cylindrical-spike model [50]. Here, the dependence of the amorphous carbon hybridization is expressed as function of the displacements per atom within the spike volume. While Robertson criticizes the lack of a physical relaxation force preferring $\mathrm{sp}^{3}$ configuration [37], the model allows the prediction of the experimental results shown, for example, by Ronning [51]. Robertson, Hofsäss and Ronning agree that the highest $\mathrm{sp}^{3}$ ratio is achieved at an ion energy of $100 \mathrm{eV}$. Towards lower energy, the $\mathrm{sp}^{3}$ content drops quickly and towards higher energies it decreases slowly. As noted by Robertson [37], the whole process is temperature dependent, elevated temperatures will also yield lower $\mathrm{sp}^{3}$ content which should be kept in mind for the track formation process. A more in-depth collection of concepts and references on the growth model is presented by Robertson.

\subsection{Stopping of ions and track formation}

\subsubsection{Stopping of ions}

Interactions of ions and solids are a facet rich phenomena. Processes are confined to small volumes in the order of cubic nanometers, energy transfers take place in short 
time spans (femtoseconds) and in case of swift heavy ions, the specific energy density is high, reaching some electronvolt per atom. The transformations of the target, induced by the ion bombardment, are often not in thermodynamically equilibrium and allow the studies of non-equilibrium physics.

In general, any particle impinging an object will lose kinetic energy due to interactions with the target. This specific energy loss, also called stopping power $S(E)$ is defined as the negative derivative of the energy over path length:

$$
S\left(E_{\mathrm{p}}\right)=-\left.\frac{\mathrm{d} E}{\mathrm{~d} x}\right|_{E_{\mathrm{p}}} .
$$

$S$ depends upon the projectile energy $E_{\mathrm{p}}$. In case of ions the units $\mathrm{eVnm}^{-1}$ or $\mathrm{eVcm}^{2}$ / atom are commonly used. If the stopping power is given per unit length, the density of the target must be known, while atoms $/ \mathrm{cm}^{2}$ is density independent unit of the stopping power.

One obvious interaction of moving ions is the collision with atoms of the target, governed by the repulsive coulomb potential of the penetrating electron clouds (see nuclear stopping power chapter 2.3.2). At sufficient speeds of the ion, the projectile is capable of ionizing the matrix along its track (see electronic stopping power chapter 2.3.3). Furthermore, at higher energies, nuclear reactions, Bremsstrahlung and Cherenkov radiation slow the projectile [10,52. Nuclear reaction takes place, if the energy of the projectile is sufficiently high to provide the required activation energy to overcome the repulsive coulomb potential of the positive nuclei. A prominent example is the reaction of ${ }^{15} \mathrm{~N}$ and $\mathrm{H}$. The $\mathrm{N}$ projectile and a $\mathrm{H}$ atom in the target can fuse to form ${ }^{16} \mathrm{O}$. The fusion energy required is approximately $6.385 \mathrm{MeV}$. This reaction is used to profile Hydrogen at low concentration and depth in thin films [53. However, the usage of swift heavy ions in the energy regime of $4 \mathrm{MeV} \mathrm{u}^{-1}$ to $6 \mathrm{MeV} \mathrm{u}^{-1}$ used in combination with carbon and silicon (substrates) targets leads to very little nuclear reactions, since the coulomb barrier is not overcome ${ }^{1}$.

In the scope of this work electronic stopping and to some part nuclear energy loss are relevant and will be examined in more detail.

\subsubsection{Nuclear stopping power}

Nuclear stopping power $S_{\mathrm{n}}(E)$ refers to the elastic collisions of the projectile with atoms of the target. Although the effect is called nuclear, the scatting potentials are not defined by nuclear forces but by the Coulomb repulsion of the ions. This interaction is dominant at the low energy regime $(<5 \mathrm{keV} / \mathrm{u})$. The cross section first increases to reach a maximum and decreases towards higher energies $10,52,54$ as it is shown for some examples in figure 2.2 (dashed lines). Each nuclear collision transfers

\footnotetext{
${ }^{1} \mathrm{~A}$ low rate of sample activation is still observed as expected due to tunneling processes (Gamow factor)
} 
a relatively large amount of energy (percent regime of the projectile energy) to the target atom, removing it from its lattice position and causing a collision cascade until the energy of all involved atoms drop underneath the threshold of the displacement energy. The series of collisions may alter the direction of the ion significantly and create a large straggling of the ions. The nuclear stopping generates the most damage to the lattice, as many atoms are displaced and statistic disorder is the result. In case of ion beam doping this effect is responsible for lattice defects which have to be healed, for example, by annealing [54].

A relatively precise method to calculate the nuclear stopping power was designed by Ziegler, Biersack and Littmark [54] using their universal screening function. This method is evaluated numerically and is implemented in SRIM described in chapter 2.3.5. Nastasi [52] and Ziegler et al. offer also theoretical descriptions of the nuclear stopping in their text books.

\subsubsection{Electronic stopping power}

The electronic stopping power $S_{\mathrm{e}}(E)$ is the result of inelastic collisions of the charged impinging ion and the electrons of the target, exciting both the target and ion electron structure. Each collision transfers a small amount of energy ( $10 \mathrm{eV}$ to $20 \mathrm{eV}$ ), while the rate of interactions is relatively large. The high number of events reduces the statistical fluctuation leading to smooth and almost continuous energy loss compared to nuclear stopping. In turn, the path of the projectile has little straggling and forms straight tracks in the target [52]. The excited electrons relax in different ways, for example, by phonon interaction, emitting a photon (typical x-ray or gamma radiation detected during ion irradiation) or leaving the atom (ionization). In addition, the number of electrons of the projectile may change as discussed in detail in chapter 2.3.4 An approach to describe the stopping power in the regime of the electronic energy loss is accomplished by the Bethe formula [52]

$$
\frac{\mathrm{d} E}{\mathrm{~d} x}=-\frac{4 \pi n z^{2}}{m_{e} c^{2} \beta^{2}} \cdot\left(\frac{e^{2}}{4 \pi \epsilon_{0}}\right)^{2} \cdot\left[\ln \left(\frac{2 m_{e} c^{2} \beta^{2}}{I \cdot\left(1-\beta^{2}\right)}\right)-\beta^{2}\right],
$$

where $\beta=v / c$ is the particle velocity, $c$ the speed of light, $E$ the energy of the particle, $z$ the charge state, $e$ the elementary charge, $n$ the electron density, $m_{\mathrm{e}}$ the electron rest mass and $I$ the average excitation potential. The excitation potential $I$ was estimated by Felix Bloch to be $I=Z \cdot 10 \mathrm{eV}, Z$ being the atomic number of the target material. More realistic results can be obtained by experimentally acquired values for $I(Z)$ as found for example by the International Commission on Radiation Units and Measurements [55].

The electronic stopping power has a maximum value, where the cross section is largest, called the Bragg peak. For carbon targets and heavy ions as $\mathrm{Au}, \mathrm{Pb}$ or $\mathrm{U}$, this energy is in the range of $4 \mathrm{MeVu}^{-1}$ to $5 \mathrm{MeVu}^{-1}$ as seen in figure 2.2. This 
maximum, is the highest stopping power available for ion track formation using these ion specimens.

\subsubsection{Charge state}

The charge of the impinging ion is called charge state and is measured in elementary charge $e$, for example, an $\alpha$ particle has a charge state of $2+$. As soon as the ion interacts with the target, the initial ionization may change. On one hand, each ion attracts electrons by the ionization potential trying to neutralize itself, on the other hand, fast ions may also be stripped of electrons, if the ion speed is in the range of the Bohr electron velocity. Both processes define an equilibrium charge state depending mainly on ion velocity. If an ion enters the target with more electrons than the equilibrium charge, it is stripped of electrons, otherwise it will gain electrons along its path. The distance until equilibrium charge is reached depends on many factors, such as target density and ion velocity.

The first theoretical descriptions of the charge state of swift ions in solids was formulated by Niels Bohr [56]. He proposed this expression for the ionization of an ion [52]:

$$
\frac{Z^{*}}{Z}=\frac{v_{\text {ion }}}{v_{0} Z_{1}^{2 / 3}}
$$

where $Z^{*}$ is the ion equilibrium charge, $Z$ the atomic number, $v_{\text {ion }}$ the ion velocity, and $v_{0}$ the Bohr electron velocity $\left(\frac{c}{137}\right)$. This expression can be improved to an empirical formula fitted to experimental results:

$$
\frac{Z^{*}}{Z}=1-\exp \left[-0.92 \cdot \frac{v_{\text {ion }}}{v_{0} Z_{1}^{2 / 3}}\right] .
$$

In a typical setup, an ion with known energy and charge state passes through thin foils called a stripper. Subsequently, the change in charge is measured ( $\frac{e}{m}$ ratio). It is important to keep in mind that the charge state is governed by statistical processes (random collisions) and is distributed with a certain width around the equilibrium. For this reason, the stopping power of otherwise two identical ions may be different. An example of the charge state distribution is shown in figure 2.3(a) in the following discussion about simulations of the stopping power.

An ion will reach its equilibrium charge after passing a certain distance in the solid. In case of sufficiently path length, the equilibrium charge state distribution can be used to estimate the stopping power. However, if effects close to the surface are analyzed, the initial charge and the change of the ionization along the path have to be taken into account. In this work, films of less than $100 \mathrm{~nm}$ thickness are irradiated and the initial charge state is of importance because the order of the ion range before reaching equilibrium is in the order of $10 \mathrm{~nm}$ to $100 \mathrm{~nm}$ [57,58. Therefore, an ion has to possess the desired charge before hitting the samples. 
In experiments, the ions are provided with a single charge state by the ion accelerator. To change the ionization state of the beam, a stripper is implemented typically realized by diluted gases or solid foils (carbon or aluminum). The stripping is essentially limited to the equilibrium charge state. If extremely high levels of ionizations are desired, very high acceleration energies are required as figure 2.3(b) illustrates.

\subsubsection{Simulation of stopping power and charge states}

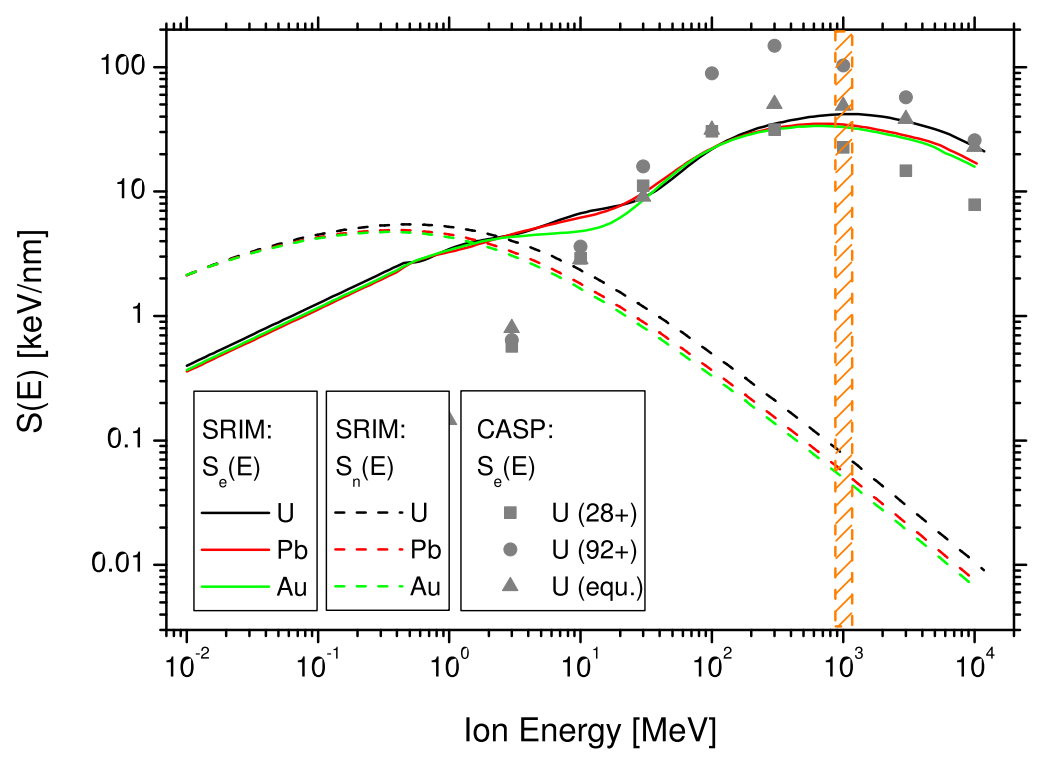

Figure 2.2: Stopping power of different simulations (SRIM and CasP). Showing the nuclear stopping at low energy regime (SRIM) and the electronic energy loss at higher energies. SRIM simulation assume equilibrium charge, whereas different charge states were simulated using CasP. At high energies, both simulations are in agreement, towards lower energies a deviation is observed. However, at the marked energy regime, where experiments were performed, both simulations are in agreement.

To design experiments and analyze results, the ion-solid-interactions can be simulated with computer algorithms. Two programs are demonstrated and used in this work. The first is SRIM [48,54. It is a versatile Monte Carlo simulation, capable of simulating the energy loss at low (nuclear stopping) and high energies (electronic stopping). Besides stopping powers, it simulates the damage cascades, sputtering etc. of the target. This software is a standard tool for predicting implantation profiles, structural damage, ion beam depositions, and straggle of ion beams within the target (proton beam writing). The second program CasP [59,60 is a specialized tool for analyzing the electronic stopping power. It allows the application of different ap- 
proximations granting the freedom of more user controlled parameters compared to SRIM, such as the charge state. On the other hand, no output for the target modifications (sputtering, displacements) and ion path (straggling) is given. Furthermore, only the electronic energy loss is modeled. Unlike SRIM, it is not a Monte Carlo simulation but an analytic calculation. Obviously, both programs have a different focus. SRIM provides essential information for sputter and implantation experiments. Its results are calibrated to experimental results leading to a high overall reliability of the predictions. CasP, on the other hand, is a theoretical approach specialized for the prediction of electronic stopping, which is dominant at higher ion energies around the Bragg peak. Further details are available online, where both programs are available free of charge $2^{2}{ }^{3}$. Unfortunately, the authors do not publish the source code.

Regarding the results of the simulations, first in figure 2.2 the stopping powers of the ions species used in this work are shown. The target material is carbon with a density of $3 \mathrm{~g} \mathrm{~cm}^{-3}$. SRIM offers an output for both $S_{\mathrm{e}}$ and $S_{\mathrm{n}}$. The nuclear stopping power (dashed lines), as expected, is dominant at low energies reaching its maximum at about $400 \mathrm{keV}$ or $2 \mathrm{keV} \mathrm{u}^{-1}$ and decreasing exponentially towards higher energies. The electronic stopping power increases with projectile energy reaching the Bragg peak at about $1 \mathrm{GeV}\left(4.2 \mathrm{MeV} \mathrm{u}^{-1}\right)$. The small orange box marks the energy regime used for the irradiations in this work. The simulation results of CasP are shown for ${ }^{238} \mathrm{U}$ and three different charge states. The triangle symbols calculated for equilibrium charge state $4^{4}$ should be in accordance with SRIM results for $S_{\mathrm{e}}$. This is the case for high energies beyond the Bragg peak. The maximum itself is shifted towards lower energies and is a little higher than the prediction of SRIM. One calibration problem is posed by the density of the material. CasP does not consider an explicit density. The results in units of $\mathrm{eVcm}^{2} /$ atom were transformed to $\mathrm{kV} \mathrm{nm}^{-1}$ by assuming a density of $3 \mathrm{~g} \mathrm{~cm}^{-3}$. SRIM takes the density as an input parameter and returns its results in $\mathrm{keV} \mathrm{nm}^{-1}$. Furthermore, SRIM is calibrated to return overall stopping powers in agreement to experimental data. To low energies, the CasP simulation returns significantly smaller values compared to SRIM. While SRIM returns a plausible overall stopping power, it is assumed, that it overestimates the electronic energy loss in the region between nuclear and electronic stopping dominance, while CasP tends to underestimate the energy loss in this regime. A comparison of both algorithms to experimental results by Singh et al. [61] shows this behavior in first order approximation. Nonetheless, at the energy regime of the experiment, both simulations yield similar results.

All irradiations in this work are done in the $\mathrm{MeV} \mathrm{u}^{-1}$ range, where both simulations agree and predict a stopping power of $30 \mathrm{keV} \mathrm{nm}^{-1}$ to $45 \mathrm{keV} \mathrm{nm}^{-1}$. In addition to

\footnotetext{
${ }^{2}$ http://www.helmholtz-berlin.de/people/gregor-schiwietz/casp_en.html

3 http://www.srim.org/

4 a weighted average of the charge state distribution was used "charge state scan".
} 
the equilibrium charge state, completely stripped $\mathrm{U}^{92+}$ ions and $\mathrm{U}^{28+}$ (as provided by accelerator) were simulated. The highly charged ion has a much higher stopping power especially in the region around the Bragg peak. In addition, the maximum is shifted towards lower energy. At higher energy the difference to the equilibrium simulation vanishes, as the equilibrium charge state approaches full ionization. The $28+$ simulation results in much lower stopping powers around the Bragg peak. The equilibrium charge state is about $60+$ at the Bragg peak. According to the Bethe formula, stopping increases quadratically with charge state as confirmed in first order approximation by the simulation.

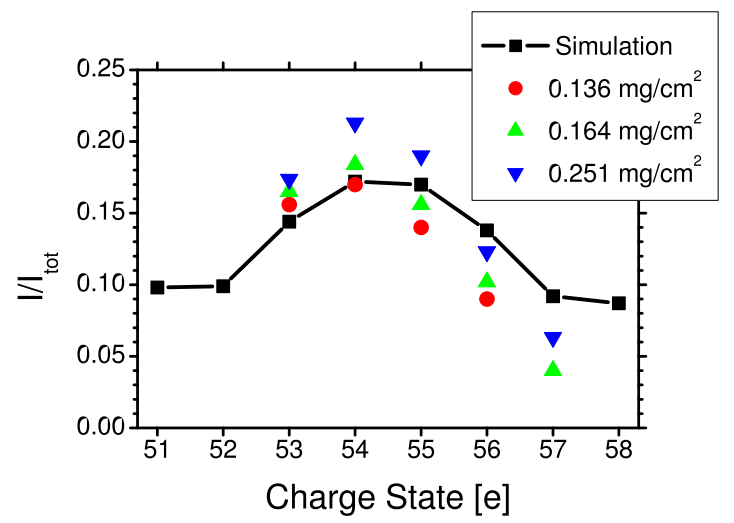

(a) Charge state distribution caused by stripper

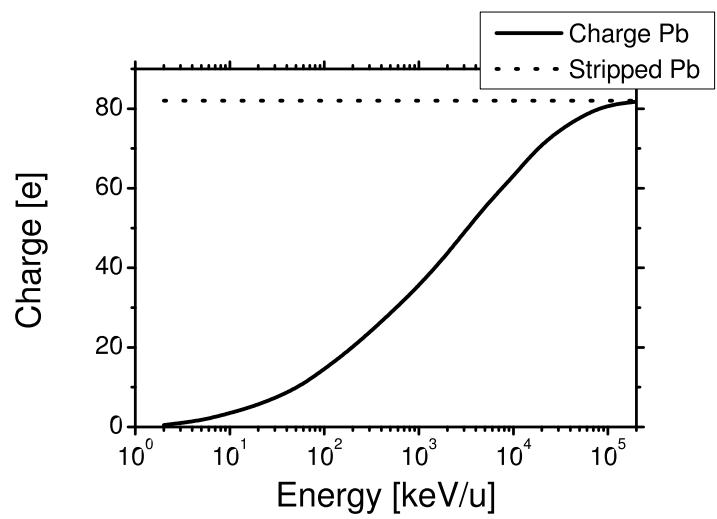

(b) Charge state depending on ion energy

Figure 2.3: (a) shows the charge distribution for $\mathrm{Pb}$ in $\mathrm{C}$ simulated with CasP (fixed charge state) and the experimental data of charge state measured after beam ionization of thin carbon foils (private communication with Omar Kamalou). The data is in good agreement with the simulation. Furthermore, the increase of higher charge state after thicker degraders suggests that equilibrium is not quite reached with the applied foil thickness. (b) shows the equilibrium charge state of $\mathrm{Pb}$ depending on its impinging energy as simulated by CasP.

Figure 2.3 $(a)$ compares the equilibrium charge state calculated by CasP to experiment. $\mathrm{A} \mathrm{Pb}^{23+}$ beam with an energy of $4.57 \mathrm{MeV} / \mathrm{u}$ is passing through thin carbon foils. After the stripping foils, the charge distribution is measured and compared to the simulation with CasP. The data $5^{5}$ shown in figure 2.3(a) reveals that both simulation and experiment yield $54+55+$ as the most likely charge states. Moreover, the width of the distributions is comparable, although the thin stripper foils are not thick enough to ionize to full equilibrium distribution. The deviation from equilibrium is visible in the increasing likelihood of higher charge states with increasing stripper thickness. The thinner foils would produce more less-charged ions, which were not recorded in this experiment. In figure 2.3(b) the weighted average of the simulated

\footnotetext{
${ }^{5}$ Private communication with Omar Kamalou. Experimental details are published by Baron and Ricaud using different ion specimens 62
} 
equilibrium charge is plotted against projectile energy. Again, the target is composed of carbon.

Uran 4.20 $\mathrm{MeV} \mathrm{u}^{-1}$ :
\begin{tabular}{l||l|l|l|l|l|l|l|} 
Charge $[e]$ & $55+$ & $56+$ & $57+$ & $58+$ & $59+$ & $60+$ & $61+$ \\
\hline Weight [\%] & 15.5 & 11.5 & 14.7 & 16.0 & 14.8 & 11.7 & 15.9 \\
$S_{\mathrm{e}}(E)\left[\mathrm{keV} \mathrm{nm}^{-1}\right]$ & 46.8 & 48.0 & 49.2 & 50.4 & 51.6 & 52.9 & 54.1
\end{tabular}

Lead 4.57 $\mathrm{MeV} \mathrm{u}^{-1}$ :

\begin{tabular}{l||r|r|r|r|r|r|r|r|r|} 
Charge [e] & $51+$ & $52+$ & $53+$ & $54+$ & $55+$ & $56+$ & $57+$ & $58+$ & $60+$ \\
\hline Weight [\%] & 9.8 & 9.9 & 14.4 & 17.2 & 17.0 & 13.8 & 9.2 & 8.7 & - \\
$S_{\mathrm{e}}(E)\left[\mathrm{keV} \mathrm{nm}^{-1}\right]$ & 39.2 & 40.3 & 41.3 & 42.5 & 43.6 & 44.8 & 46.0 & 47.2 & 49.6
\end{tabular}

Gold 5.20 $\mathrm{MeV} \mathrm{u}^{-1}$ :

\begin{tabular}{l||r|r|r|r|r|r|r|r|} 
Charge $[e]$ & $50+$ & $51+$ & $52+$ & $53+$ & $54+$ & $55+$ & $56+$ & $57+$ \\
\hline Weight [\%] & 6.7 & 8.0 & 12.8 & 16.6 & 17.7 & 15.5 & 11.1 & 11.5 \\
$S_{\mathrm{e}}(E)\left[\mathrm{keV} \mathrm{nm}^{-1}\right]$ & 35.4 & 36.4 & 37.4 & 38.4 & 39.5 & 40.5 & 41.6 & 42.7
\end{tabular}

Table 2.1: Lists of electronic stopping powers for three different ions in carbon. All values were simulated with CasP, the charge states were chosen from the equilibrium distribution simulated by CasP as well (except $\mathrm{Pb}^{60+}$ ). The energy deposition of the same ion-type varied by $15-20 \%$ in equilibrium.

Finally, table 2.1 shows the charge state distributions and the simulated stopping power in carbon of three ion types. The relatively wide distribution of charge states and the resulting fluctuation of stopping powers become obvious. The simulated charge states are part of the predicted equilibrium distribution. The chosen ion species and energies resemble the beam properties available for this work. The variation in stopping power will be significant for the track formation.

\subsubsection{Track formation}

The energy lost by the projectiles is deposited along the path of the ion. The energy is capable, depending on target material, to trigger transformations, which in turn form tracks. A typical effect can be the amorphization of a crystalline target (amorphous ion track) or the change of chemical bonds in polymers (latent track 6 ).

The first ion tracks noticed were fission tracks in LiF observed by Young [4] and in Mica analyzed by Silk and Barnes [5]. The tracks in LiF become only visible after chemical etching. The disordered structure along the track trajectory has a higher etching rate and a microscopic crater appears for each track as seen in figure 2.4(a). Using a modern swift heavy ion accelerator at the GSI, Trautmann et al. analyzed

\footnotetext{
${ }^{6}$ The tracks are 'invisible', but can be etched selectively.
} 
the damage caused in LiF to more detail [9]. Using multiple ion specimens, resulting in different energy loss and material damage, they could show a threshold $S(E)$. Lower energy loss will not produce etchable tracks. The cross section of the sample shows the length of the ion tracks, which is in the order of $50 \mu \mathrm{m}$ for swift heavy ions in the $\mathrm{GeV}$ range. Finally, the confirmation of nuclear energy loss causing substantial structural damage in LiF, Trautmann et al. examined a cross section of an irradiated crystal. Here, etching damage was only observable at the end of the ion range, where the slowed ions exhibit mainly nuclear energy loss. The influence of the changing stopping power and the different form of resulting ion track is shown by Herre et al. in InP by Xe irradiation [63]. As the ion slows down, $S(E)$ can be transformed to $S(r)$, where $r$ is the ion range. This quantity was simulated by Herre et al. for electronic energy loss (ionization) and nuclear stopping power (displacements). In figure 2.4(b) the results are shown for $250 \mathrm{MeV}$ Xe in InP. The electronic stopping monotonously decreases along the ion path, while the nuclear stopping increases at greater depth reaching its maximum directly before finally stopping.

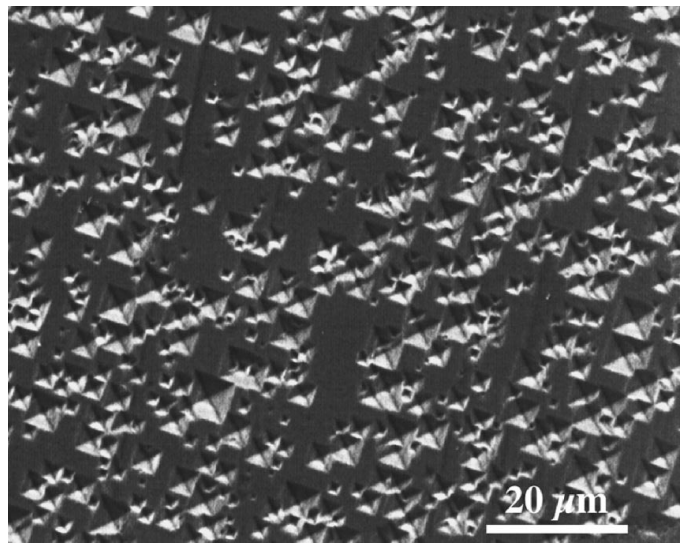

(a) Tracks in LiF (taken from Trautmann et al. [9])

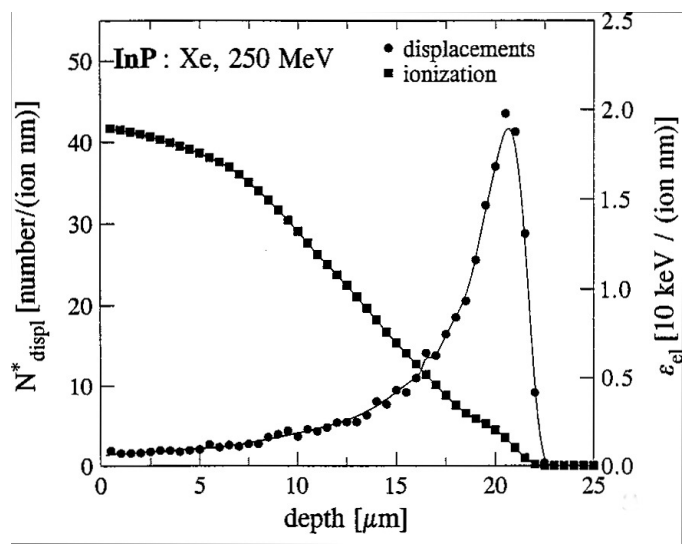

(b) Displacement and Energy loss along ion track (taken from Herre et al. 63])

Figure 2.4: (a) shows the etched latent tracks in LiF. Each crater indicates an individual ion track. The change of electronic and nuclear stopping along the path as simulated in $\mathrm{InP}$ for $\mathrm{Xe}$ ions is shown in (b). The electronic energy loss decreases monotonously while the nuclear stopping reaches a maximum just before completely stopping.

These exemplary ion track experiments demonstrate the most important factors for ion track creation. The type of track depends on target type (material) and energy loss of the ion. As shown in figure 2.2, the ion energy influences significantly the stopping power. Commonly, a threshold energy density must be deposited to form tracks, the ion specimen itself is usually of less importance mostly $S(E)$ is relevant. The tracks formed by nuclear and electronic stopping can be different as seen in the example of InP [63]. 
Theoretically, the diameter of the initial energy deposition, equivalent to the volume of the directly exited electron system, can be estimated by the Bohr adiabatic radius:

$$
r_{\mathrm{ad}}=\frac{v}{\omega_{0}}
$$

where $v$ is the ion velocity and $\omega_{0}$ the plasmon frequency of the target material. For swift heavy ions this radius is in the order of $1 \mathrm{~nm}$. Therefore, the energy density in this initially exited volume reaches very high values of $200 \mathrm{eV}$ to $300 \mathrm{eV}$ per atom. Furthermore, the energy transfer from the projectile to the target takes place in very short time frames in the order of $1 \times 10^{-17} \mathrm{~s}$ as the particles travel with about $10 \% \mathrm{c}$. Within the first $1 \times 10^{-14} \mathrm{~s}$, an electron cascade transports the energy outward. The initial track becomes wider and the energy density decreases. By electron-phononinteraction, the energy is passed to the matrix. The time frame for this process is still short taking place in the order of $1 \times 10^{-12} \mathrm{~s}$. Finally, the system cools rapidly (quenching), freezing the ion track. Depending on heat transfer, this process is in the order of $1 \times 10^{-12} \mathrm{~s}$ to $1 \times 10^{-10} \mathrm{~s}$. Typical tracks are in the order of $5 \mathrm{~nm}$ to $10 \mathrm{~nm}$ wide as shown for ta-C by Schwen [22] or for $\mathrm{SiO}_{2}$ by using SAXS by Kluth et al. 64.

The final track diameter and the track formation process as a whole can be estimated by modeling and simulation. For macroscopic calculations the concepts of Coulomb explosion [65 67] and thermal spike 68 70 are used. While microscopic approaches are based on molecular dynamic simulations [71, 72], and for ta-C by Schwen [22, 23], the thermal spike is described by two coupled equations [73]:

$$
\begin{aligned}
C_{\mathrm{e}} \frac{\partial T_{\mathrm{e}}}{\partial t} & =\nabla\left(K_{\mathrm{e}} \nabla T_{\mathrm{e}}\right)-g\left(T_{\mathrm{e}}-T\right)+B(r, t) \\
\rho C(T) \frac{\partial T}{\partial t} & =\nabla(K \nabla T)+g\left(T_{\mathrm{e}}-T\right),
\end{aligned}
$$

where $C$ is the specific heat, $K$ the thermal conductance, $\rho$ is the specific mass of the lattice, $g$ is the electron phonon coupling constant, and $B(r, t)$ is the energy density supplied by the incident ion to the electronic system by ballistic collisions. The subscript 'e' indicates values of the electrons, the non-subscript variables describe the atomic system. Toulemonde et al. applied the thermal-spike model to $\alpha-\mathrm{SiO}_{2}$ and calculated the temperature in dependence of the time. Furthermore, the track diameter is estimated by assuming track formation within the volume heated above the melting temperature compared to experimental results. The model yields good correlation to experimental results; the diameters are again in the order of $5 \mathrm{~nm}$ to $10 \mathrm{~nm}$, depending on energy loss of the impinging ion [73]. Furthermore, the time scales are in agreement with the theoretical expectations.

Another aspect has to be considered for track formation at lower stopping powers close to the threshold of formation. The ionization processes within the very small volumes and short time spans responsible for the energy loss are finite in number. 
Since each collision is a random process a fluctuation of the material transformation along the ion path is plausible. This concept was applied to Muscovite Mica by Dartyge and Sigmund [74 and to Yttrium iron garnet by Meftah et al. [75]. Both publications describe a transition from discontinuous track fragments to fully developed tracks with increasing energy loss of the irradiation ion. In other words, at the threshold for track formation, the material transition is not complete and more developed tracks will form at higher stopping powers until a complete transformation is achieved and the track properties are almost independent of energy loss 7 . Inhomogeneous tracks can be seen in LiF (figure 2.4(a)), where the etched craters are different in size. Therefore, it is often desired to maximize the energy density to produce uniform pronounced tracks. In addition to the stopping power, which is maximized at the Bragg peak, the ion velocity has an effect. According to equation 2.5 the excitation radius is proportional to the velocity which should be kept as low as possible. Because of this velocity effect, it is often efficient to choose the left side of the Bragg peak for ion track formation [76,77.

The field of application of ion tracks is large. Besides fundamental investigations of non-equilibrium physics, the tracks can be used to engineer nanodevices. Some examples are the creation of porous polymers which in-turn can be used as masks to produce nanowires [78], field emission structures [14, 15]. More applications and effects are discussed by Spohr [10]. An overview of current research taking place at the GSI is summarized in its annual report of 2011 [79].

\subsubsection{Track formation in ta-C}

Swift heavy ion irradiation of ta-C leads to a unique increase in conductivity of the material. Many amorphous insulators increase their conductivity after swift heavy ion irradiation because of a generally increasing defect density [11]. However, in case of ta-C , the current increases by many orders of magnitude. The change of electric properties is confined to the track volume and the high contrast in conductivity leads to embedded nanometer-sized filaments called conductive ion tracks 26, 28, 80. By atomic force microscopy, it is possible to visualize these tracks directly as shown in figure 2.5. Each ion leaves two signs visible by atomic force microscopy (AFM). The ion track is marked by a hillock (few nanometer hill) and a locally increased conductivity. The hillocks are found by traditional AFM analysis of the surface topography (figure 2.5(a)). The current measurement requires a conductive tip and a special AFM capable of measuring the tip current as a bias voltage is applied to the sample (see chapter 3.6). The result is a current-map of the area scanned. The data shown in figure 2.5 is taken from the same area and each hillock corresponds to a spot of high conductivity marking an individual track.

The formation of ta-C films is achieved by ion beam deposition, where the energy

\footnotetext{
${ }^{7}$ The radius will still increase.
} 


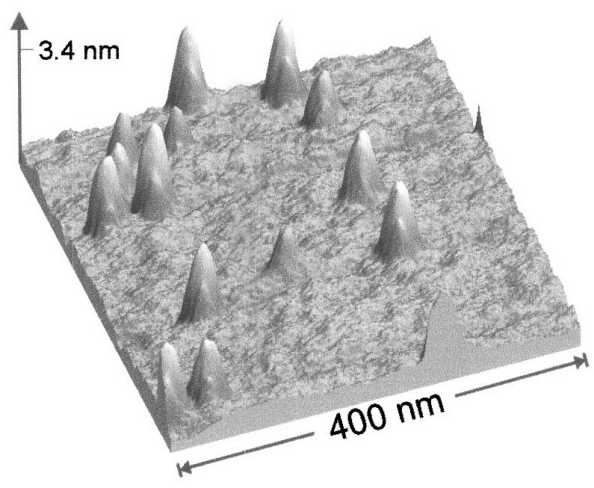

(a) Topography of ion tracks in ta-C

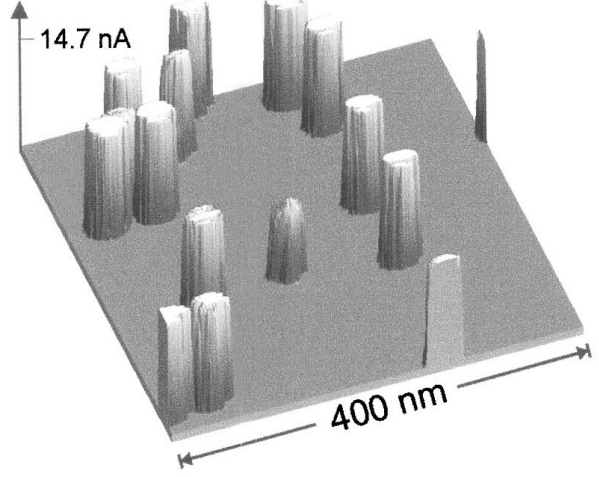

(b) Current map of ion tracks in ta-C

Figure 2.5: AFM scans of ion tracks in ta-C. (a) shows the topography of the flat film with hillocks for every track while (b) displays the current measured of the same location. Each track exhibits an elevated current. (taken from Krauser et al. [28])

has to be within a small margin to yield high $\mathrm{sp}^{3}$ bond ratio. This requirement, already suggests a metastability of the $\mathrm{sp}^{3}$ bond in a-C. In fact, the high $\mathrm{sp}^{3}$ bond ratio will decrease at high temperatures. More precisely, the activation for the $\mathrm{sp}^{3} \rightarrow$ $\mathrm{sp}^{2}$ reaction is about $3.3 \mathrm{eV}$ to $3.5 \mathrm{eV}$ as measured by Raman Spectroscopy (Ferrari et al. [81]) and X-ray Absorption Near Edge Structure (by Grieson et al. [82]). A $\mathrm{sp}^{2}$-rich DLC film has a higher conductivity, as well as a smaller optical gap [37, 83]. The energy loss of swift heavy ions is sufficient to activate the transformation towards $\mathrm{sp}^{2}$ bonds within the confined track volume leaving conducting ion tracks in $\mathrm{sp}^{3}$-rich amorphous carbon matrix upon irradiation.

The track formation is illustrated in figure 2.6. The impinging ion hits the $\mathrm{sp}^{3}$-rich ta-C with about $5 \%$ to $10 \%$ of the speed of light. It deposits the energy within $3 \times 10^{-15}$ s along a typical length of $100 \mathrm{~nm}$ mainly as electronic stopping power. The energy spreads within the the next $1 \times 10^{-14} \mathrm{~s}$ activating the bond transformation creating a more $\mathrm{sp}^{2}$-rich track. The lesser density of the $\mathrm{sp}^{2}$ bond carbon increases the pressure inside the track. During the relaxation the typical hillock is formed as seen by AFM measurements. The complete track formation is finished within $1 \times 10^{-12} \mathrm{~s}$ to $1 \times 10^{-10} \mathrm{~s} 222,23$.

The final track diameter is about $8 \mathrm{~nm}$ as measured by TEM [22, 28]. Assuming an energy loss of $40 \mathrm{keV} \mathrm{nm}^{-1}$ results in an average energy deposition of about $5 \mathrm{eV}$ per atom. Simulations by Schwen et al. [23] suggest temperatures in the order of $2 \mathrm{eV}$ to $2.5 \mathrm{eV}$, corresponding to measurements of Auger-electrons [84]. The time scale for the hillock formation is estimated by these simulations to be in the order of $1 \times 10^{-11} \mathrm{~s}$, being in time frames generally assumed for track formation in amorphous materials. Furthermore, Schwen et al. showed an increase of hillock height with growing energy 
loss in the regime of $20 \mathrm{keV} \mathrm{nm}^{-1}$ to $80 \mathrm{keV} \mathrm{nm}^{-1}$ 22, 23. This correlation has been observed by Waiblinger et al. [80] and Krauser et al. [24]. Furthermore, a threshold stopping power of $10 \mathrm{keV} \mathrm{nm}^{-1}$ to $20 \mathrm{keV} \mathrm{nm}^{-1}$ is required for track formation 23,26 , 80].

The material in the track volumes, created with stopping powers close to the threshold, is not completely transformed. Schwen et al. suggest a linear relationship between energy loss and hillock height at least up to $80 \mathrm{keV} \mathrm{nm}^{-1}$ [23. In this case, the saturation described by Herre et al. for complete track formation lies beyond $80 \mathrm{keV} \mathrm{nm}^{-1}$ and beyond the stopping powers available through swift heavy ions. Therefore, the track formation in ta- $\mathrm{C}$ is sensible to small fluctuations in energy loss under typical irradiation conditions. The charge state distribution received by stripping the beam by aluminum foils produces a fluctuation of stopping power of about $20 \%$ (table 2.1). In addition, the statistical nature of the track formation process will cause a distribution of track properties even in the event of perfectly constant stopping powers. The electronic properties depend stronger than the hillock height on the degree of transformation within the track volume. A significant fluctuation of the conductivity of even neighboring ion tracks is always observed in irradiated ta-C $22,24,25,33,36]$.

This fluctuation of track, conductivity eventually caused by random processes, is a draw back of conductive ion track usage in devices. The only method to remove this fluctuation is the usage of very high stopping powers surpassing the maximum possible with heavy ions. A possibility is the usage of molecule irradiation, such as C-60 yielding uniform tracks [24,25]. However, the path-length until these fullerens break up and spread are in the order of $100 \mathrm{~nm}$ [85]. Furthermore, these C-60 beams are even rarer than swift heavy ion beams.

A different approach is the increase of the initial $\mathrm{sp}^{2}$ content as done by Zollonz et al. 26] and adding impurities to the ta-C . Both approaches increase the track conductivity and the unirradiated matrix. In most cases the matrix conductivity increases more reducing the overall contrast. Nevertheless, higher conductive tracks could be interesting for applications even if embedded in a less resistive matrix. One goal of this thesis is the search for an optimal copper impurity concentration.

\subsubsection{Conductive tracks in other materials}

Many materials, especially amorphous materials, may show an increased conductivity upon irradiation, because the defect density is raised. However, in most cases the change in conductivity is small compared to the change observed in ta-C. Notable are some experiments on polymers regarding carbonization and break-down voltages 86, 87.

Other carbon based materials exhibiting conductive tracks are C-60-films [88]. The origin of the track conductivity is probably similar to DLC, where the deposited energy causes defects changing the bond structure of the carbon. Although, theoret- 


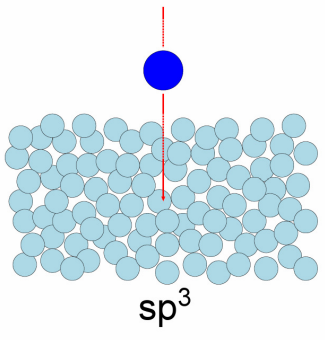

(a) Ion hitting ta-C

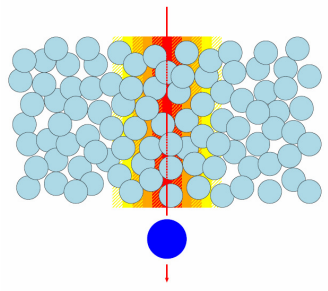

(b) Energy deposition

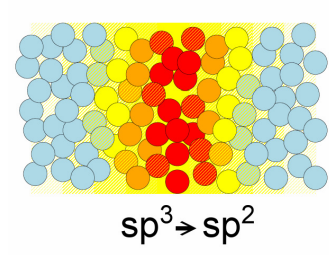

(c) Track formation

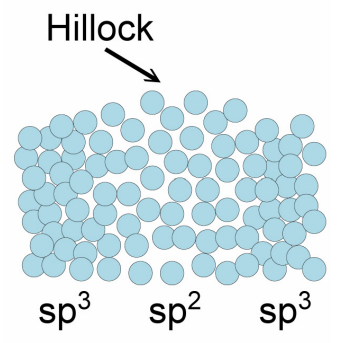

(d) Track relaxation

Figure 2.6: The above figures (adopted from Schwen [22]) illustrate the track formation process. After the ion passes the thin film in about $3 \mathrm{fs}$. The deposited energy first cascades through the electron system of the target $(1-10 \mathrm{fs})$, than couples to the lattice $(0.1-200 \mathrm{ps})$. The energy per atom can be estimated to be $5 \mathrm{eV}$ within the track volume.

ically diamond would be a candidate for conductive ion tracks, the crystalline matrix is too stable to be transformed by the energy density possible with swift heavy ions. In fact, diamond anvil cells are very inert to radiation damage even at extreme pressures and after high fluences [89]. Although diamond can be damaged by irradiation, no track formation by electronic energy loss is observed; the damage is the result of nuclear stopping or cascades of the swift heavy ions and not the result of one individual ion.

Another material, where a conductivity change induced by swift heavy ions is observed, is $\mathrm{VO}_{2}$ analyzed by Ehrhardt [90] and Hofsäss et al. 91]. $\mathrm{VO}_{2}$ exhibits a metal-semiconductor transition around $60^{\circ} \mathrm{C}$. The low temperature semiconductor phase shows improved conductivity upon swift heavy ion irradiation. Above the transition temperature, the irradiation effect on conductivity vanishes. So far, no individual tracks with typical diameters of some nanometer could be observed. The $\mathrm{VO}_{2}$ films observed were micro-crystalline. Hofsäss et al. and Ehrhardt suggest an effect of a single ion to a whole grain.

A change in conductivity was also observed after the irradiation of $\mathrm{CuIr}_{2} \mathrm{~S}_{4}$ with $\mathrm{H}$ and $\mathrm{He}$ ions at low temperatures 92 . These tracks are only stable at low temperatures well below room temperatures. The energy density required is much smaller, the transition can be caused by x-ray photons as well. 


\subsection{Electron transport mechanisms}

\subsubsection{Fundamental concepts}

The two fundamental quantities in electrical transport are current $I$ and the potential $V$. The current, measured in $\mathrm{A}$, is equivalent to the flux of charge per time $(1 \mathrm{~A}=$ $\left.1 \mathrm{C} \mathrm{s}^{-1}\right)$. The potential describes the energy per charge $\left(1 \mathrm{~V}=1 \mathrm{~J} \mathrm{C}^{-1}\right)$. The product of current and potential is equivalent to the energy transported by the current. A third fundamental quantity is provided by the quotient of current and potential:

$$
R=\frac{V}{I} \text { and } G=\frac{I}{V}
$$

Here $R$ is the resistance in $\Omega$ and $G$ is the conductance in $\mathrm{S}$ (Siemens). $R$ and $G$ are the reciprocal of each other and provide the same information. In general, the resistance is a function of potential (or current). If, however, $R(V)=$ const., $I$ is proportional to $V$, the behavior is called ohmic-behavior in reference to Ohm's law 93 :

$$
U=R I
$$

The resistance does not only depend on material properties but also on sample and contact geometry. To obtain quantities independent of geometry, specific resistivity $\rho$ and specific conductivity $\sigma$ are defined:

$$
\rho=\frac{V}{I} \frac{A}{d} \text { and } \sigma=\frac{I}{V} \frac{d}{A},
$$

where $A$ is the cross section area of the conductor and $d$ the length (or thickness). Again, both quantities are reciprocal to each other. In case of an ohmic material, $\rho$ and $\sigma$ are constant, generally they depend on $V$. In the same fashion, $I$ and $V$ are normalized as well:

$$
J=\frac{I}{A} \text { and } F=\frac{V}{d}
$$

where $J$ is the current density and $F$ is the electric field $(V / d)$. Note, $F$ is used for the electric field instead of standard $E$ to distinguish electric field and energy. Ohms law can be written in terms of specific quantities:

$$
J=\sigma F=\frac{1}{\rho} F
$$

\subsubsection{Transport of electrons}

The discovery of the electron by Thomson [94] in 1897 marked the beginning of the modern understanding of charge transport. An early model describing the current transport behavior in metals is the Drude model [95] from 1900. This model assumes weakly bound valence electrons in metals, which form a "sea" of electrons called a 
free electron gas. These electrons are accelerated by the external electric field $F$. The resistivity is caused by collisions of the drifting electrons with phonons and lattice defects. The material specific properties for this theory are the electron density $n$ and the mean time between collisions $\tau$. The current density is equivalent to

$$
J=v_{\mathrm{D}} n q=\mu n F=\frac{q^{2} \tau n}{m_{\mathrm{e}}} F,
$$

where $m_{\mathrm{e}}$ is the electron mass, $q$ the electron charge $\left(-1.602 \times 10^{-19} \mathrm{C}\right)$ and $v_{\mathrm{D}}=$ $-\frac{\tau q F}{m_{\mathrm{e}}}$ is the drift speed. The drift speed is the electron velocity caused by the electric field excluding the thermal velocity. In an engineering context and semiconductor physics $\mu=\frac{v_{\mathrm{D}}}{F}$, the electron mobility is used instead of drift speeds. Increasing its value is often related to lowering defect densities and effective conduction in semiconductor devices. The Drude model obeys Ohm's law as the current is proportional to $F$. Further details and the historic impact of this model are described, for example, by Kittel [96] and Ashcroft [97] in their solid state physics text books.

In the decades following the Drude model, the fundamentals of quantum mechanics were discovered and formulated. The behavior of electrons in solids is majorly affected by quantum mechanical principals. While the Drude model is sufficient to describe the electron transport in metals, it is inadequate to understand the transport in semiconductors and amorphous insulators. Here, the concept of electrons drifting in quasi free electron gases is not applicable.

In order to understand the transport mechanism in non-metalic materials, the concept of band structure is essential. The band structure can be seen as a result of the Pauli-Exclusion Principal stating that no two fermions (e.g. electrons) can have the same wave-function (state) [96, 97]. Each state is characterized by an energy. The electrons prefer states with lower energies filling up all states up to a specific energy called Fermi energy $\left(E_{\mathrm{F}}\right)$. In bulk solids the large number of states form quasi continuous bands with respect to energy. The amount of states in a small energy range $\mathrm{d} E$ is called density of states (DOS) $D(E)$. At certain energy ranges, $D(E)$ is zero (forbidden zones) which are called band gaps. The electric properties of a solid are substantially governed by the band structure close to $E_{\mathrm{F}}$ and the band gap. When the Fermi energy lies within a band it is called a conduction band exhibiting metallic conduction (covered by the Drude model). If, however, the Fermi energy is between two bands in a band gap, the material is classified as a semiconductor or insulator. At $0 \mathrm{~K}$, no electron migration is possible because an electron trying to change its position cannot find a free state at the same energy level, since all states are occupied. The resistivity is infinite.

At finite temperatures, the thermal activation energy $k_{\mathrm{B}} T$ excites some electrons into more energetic states. In classical physics, thermal distributions are described by the Maxwell-Boltzmann statistics. In case of fermions (electrons), this approach leads to incorrect results. Instead, the Fermi-Dirac statistics must be applied to 
a) metallic:

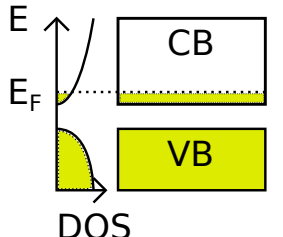

b) intrinsic:

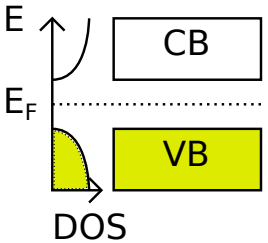

C) n-doped:

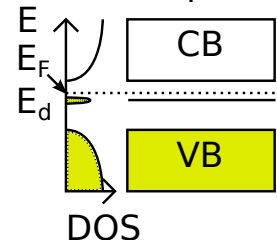

d) p-doped:

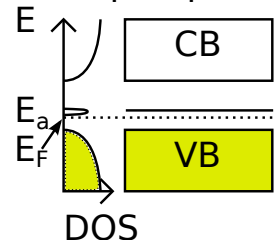

e) amorphous:

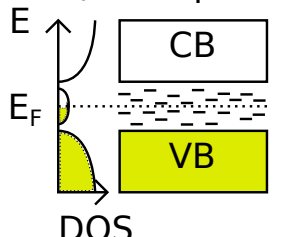

Figure 2.7: Simplified schematics of band structures for different classes of conductors. For each system the valence band (VB) and conduction band (CB) are shown as well as an exemplary plot of the density of states (DOS). As dotted line, the Fermi energy $E_{\mathrm{F}}$ is plotted. In metals (a) the Fermi energy is within the conduction band and free electrons for current transport are available at any temperature. In case of an intrinsic (b) semiconductor, $E_{\mathrm{F}}$ is in the middle of the band gap. Electrons must be exited over the whole band gap to reach the conduction band. Next an n-doped (c) semiconductor is shown. Additional donor states are created within the band gap below the Fermi energy. The excitation required is much reduced in comparison to the intrinsic case. Analogously, the p-doped (d) semiconductor has acceptor states close to the valence band. The excitation energy required to lift electrons into these acceptor states is again much reduced compared to band gap excitations. In case of an amorphous semiconductor (e), new states are created within the band gap which are strongly localized. Instead of drifting, electrons migrate by hopping.

calculate the occupation probability at energy $\tilde{E}$ :

$$
f(\tilde{E})=\frac{1}{\exp \left(\frac{\tilde{E}-E_{\mathrm{F}}}{k_{\mathrm{B}} T}\right)+1} .
$$

At $T=0$, this distribution has a sharp step at $E_{\mathrm{F}}$, states below this energy are occupied, all others are empty. As the temperature raises, the transition becomes smoother, where $f\left(E_{\mathrm{F}}\right)=0.5$ and states below and above the Fermi energy are occupied with a finite probability. The transition width grows with $k_{\mathrm{B}} T$.

The number of occupied states at a given temperature and energy is the product of the Fermi-Dirac statistic and the density of states:

$$
N(\tilde{E}, T)=f(\tilde{E}, T) \cdot D(\tilde{E}) .
$$

The total number of occupied states $N$ is equivalent to the integral over all energies:

$$
N=\int_{0}^{\infty} N(\tilde{E}, T) \mathrm{d} \tilde{E}=\int_{0}^{\infty} f(\tilde{E}, T) \cdot D(\tilde{E}) \mathrm{d} \tilde{E}
$$

In case of amorphous semiconductors, the density of states close to the Fermi energy is an important quantity it is called $N\left(E_{\mathrm{F}}\right)$ (see chapter 2.5.2).

In semiconductor, where $E_{\mathrm{F}}$ is within the band gap, the smearing of the FermiDirac distribution predicts a finite probability of occupied states within the conduction band. Similarly to Drudes concept, the thermally activated electrons can move freely in this partially filled band transporting a current. In addition, each 
activated electron leaves a void in the valence band, which enables the electrons here to migrate as well. In semiconductor physics, this process is referred to as hole conduction. If the activation energy is large enough $\left(E_{\mathrm{A}}=E_{\mathrm{C}}-E_{\mathrm{F}} \gg k_{\mathrm{B}} T\right)$, the Fermi-Dirac statistic is simplified to $f\left(E_{\mathrm{C}}\right) \approx \exp \left(\frac{E_{\mathrm{F}}-E_{\mathrm{C}}}{k_{\mathrm{B}} T}\right)$. The exponential decay of $f(E)$ allows estimating the amount of electrons in the conduction band to be proportional to $f\left(E_{\mathrm{C}}\right)$. In turn, the conductivity of the system is proportional to the number of electrons in the conduction band:

$$
\sigma \propto \exp \left(-\frac{E_{\mathrm{A}}}{k_{\mathrm{B}} T}\right)
$$

Combining equation 2.17 with the Drude model equation 2.13 leads to

$$
J=\mu n_{0} \exp \left(-\frac{E_{\mathrm{A}}}{k_{\mathrm{B}} T}\right) F,
$$

where $n_{0}$ is the DOS at the band edge. In case of an intrinsic semiconductor, where the Fermi-energy is in the middle of the band gap, the activation energy can be written as $E_{\mathrm{A}}=\frac{1}{2} E_{\mathrm{C}}-E_{\mathrm{V}}$ independently of the Fermi energy. This intrinsic approximation is often valid for undoped semiconductor and insulator materials.

The theoretical concepts described in this section are covered by many text books on solid state physics including Ashcroft [97], Ibach-Lüth [98] and Kittel [96]. In the following sections the conduction mechanisms, which are relevant to the analysis of this work are described, focusing on conduction in disordered solids as ta-C.

\section{Metallic conductivity}

As mentioned above, metallic conduction can be described by the Drude model. Although Drude uses a solely classical approach, without regards to band structures and quantum states, his model is surprisingly successful. The reason lies mainly in the weakly bond valence electrons forming the conduction band as an almost free electron gas. The dispersion of these electrons is well approximated by classical dispersion. In semiconductors the dispersion has to be corrected by the concept of effective mass [97, 98]. The collisions limiting the electron mobility are governed by phonon and impurities (defects in the lattice). The latter is almost temperature independent, whereas the phonon interaction vanishes at $0 \mathrm{~K}$ and increase exponentially to higher temperatures as described by the Debye model [99] and Einstein model [100] of the specific heat in solids. (These theories are covered by text books as well [96 98].) The mobility of the electrons in metal can be described by the sum of both collision cross sections:

$$
\frac{1}{\mu(T)}=\frac{1}{\mu_{\mathrm{p}}(T)}+\frac{1}{\mu_{\mathrm{d}}},
$$

where $\mu_{\mathrm{p}}$ is the phonon- and $\mu_{\mathrm{d}}$ the defect-contribution . At low temperatures, the phonons vanishes and $\mu_{\mathrm{p}}$ approaches infinity. The total mobility $\mu$ is at a maximum 
at low temperatures. Towards higher temperatures, the interaction with phonons increases and the overall mobility decreases. Therefore, looking at equation 2.13, the resistivity of a metal decreases towards low temperatures reaching a minimum (residual resistivity), which depends on the quality of the crystal. An example of this behavior is presented for $\mathrm{Na}$ by Ibach and Lüth 98 portraying the characteristic $T^{5}$ dependence at very low temperatures and the effect of defect density. At higher temperatures the dependence on temperature is weaker and can be approximated by linear empirical formulas as often done in technical descriptions of metal conductivities [98].

The temperature dependence of the conductivity of metals (metallic conduction) can be expressed as a function of two contributions:

$$
\frac{1}{\sigma(T)}=\frac{1}{\sigma_{\mathrm{p}}(T)}+\frac{1}{\sigma_{\mathrm{d}}}
$$

The $I$ - $V$-behavior of metals is ohmic because the number of charge carriers is mutually independent of the field. Experimentally, metallic conduction at moderate and high temperatures can be identified by ohmic characteristics and a small negative temperature dependence. In the scope of this work, metallic conduction is expected in contact wires and evaporated contact pads.

\subsubsection{Crystalline semiconductors}

In crystalline (undoped) semiconductors the temperature dependence of the electron transport is ultimately governed by equation 2.18. The electron mobility is similarly influenced as in metals resulting in higher mobilities at lower temperatures. However, unlike metals, the number of free electrons depends on thermal activation. The exponential nature of this activation usually outweighs the change in mobility. Therefore, contrary to metals, conductivity increases with raising temperature. Nonetheless, improving electron mobility in crystalline semiconductors is an important task of semiconductor engineering [98]. The activation energy in undoped intrinsic semiconductors is $E_{\mathrm{A}}=\frac{1}{2} E_{\mathrm{g}}=E_{\mathrm{C}}-E_{\mathrm{V}}$ as the Fermi energy is in the middle of the band gap.

Besides reducing the mobility, impurities have an additional influence on conductivity in semiconductors. Each impurity modifies the band structure introducing new states inside the band gap. If a newly introduced site is occupied (below $E_{\mathrm{F}}$ ) it is called donor, else it is an acceptor as illustrated in figure 2.7. In silicon, boron and phosphorus are effective acceptors (p-type) and donors (n-type). At finite temperatures, the required activation energies for exciting electrons from donor levels to the conduction band or from valence band to acceptor levels are significantly reduced compared to the intrinsic case. The extrinsic donor or acceptor conduction is described again by equation 2.18, where $n_{0}$ is the number of dopant sites and $E_{\mathrm{A}}$ is the distance from doping level to the band edge. At high temperatures, all doping sites 
are exited and the thermal activation is limited by the number of available states. In this exhaustion- or saturation-regime the conductivity is independent of temperatures. At sufficiently high temperature, the intrinsic activation becomes dominant. In this work only highly doped silicon is used at moderate temperatures, exhaustion is not expected to be relevant in the scope of this work. Further details on theoretical description of doped crystalline semiconductors can be found in text books [96 98].

\subsubsection{Asymmetric conduction - Schottky contact}

The doping of semiconductors changes the position of the Fermi energy because the introduction of new states change the maximum energy required to occupy all electrons. Common devices depend on the combination of differently doped semiconductors, as for example a $p$ - $n$ junction. A n-doped (donor) and a p-doped (acceptor) semiconductor are joined. The Fermi level is usually different in both materials. A migration of charge carriers close to the junction driven by the potential difference of the Fermi energy is the result. This redistribution locally changes the occupancy of the states forming two oppositely charged regions. The positivity charged area is depleted of electrons essentially causing the high resistance of p-n-diodes in reverse polarity 98 . A similar effect can be observed in certain metal-semiconductor junctions. The high electron mobility in metals conduction bands prevent the formation of locally confined charged volumes. However, these volumes are still found within the attached semiconductor. If a depletion region is formed a measurable rectifying property of the junction is observed. This phenomena is referred to as Schottky contact or Schottky diode [98].

The conductivity through p-n junctions and Schottky contact can be modeled by the Shockley equation [101]:

$$
I=I_{\mathrm{s}}\left[\exp \left(\frac{V q}{\tilde{n} k_{\mathrm{B}} T}\right)-1\right]
$$

where $I_{\mathrm{s}}$ is the saturation current and $\tilde{n}$ is the ideality factor. The ideality factor is 1 for an ideal p-n diode without any recombination and $1<\tilde{n}<2$ for junctions with recombination.

In case of Schottky contact (Schottky diode), the current in reverse bias is described by Shottky emission 102]:

$$
J \propto A^{*} T^{2} \exp \left[\frac{-q}{k T}\left(\phi_{\mathrm{sh}}-\sqrt{\frac{q F}{4 \pi \epsilon_{0} \epsilon}}\right)\right],
$$

where $A^{*}$ is the effective Richardson constant and $\phi_{\mathrm{sh}}$ is the barrier height for the charge carriers at the interface.

Schottky diodes can be the desired or undesired result of metal contact on-top of semiconductor surfaces. An in-depth description and discussion of such diode behav- 
ior in case of metal contacts on-top of ta-C films deposited on various semiconductors is given by Brötzmann 103$]$.

\subsubsection{Tunnel current}

In addition to the band structure, quantum mechanics predicts for small particles the possibility to tunnel through potential barriers. This phenomena is essentially the result of the decay of the wave-function $\Psi(t, x)$ inside a finite barrier. If the thickness of the barrier is sufficiently small, the particle probability at the other side is proportional to $\Psi(t, x)^{2}$. Obeying the boundary conditions for the wave-function, a transmission rate $\tilde{t}=\frac{n_{\mathrm{t}}}{n_{\mathrm{i}}}$, the quotient of transmitted and impinging particles, can be expressed depending on barrier-height $\phi$ and particle energy $E$ :

$$
\tilde{t}^{2}=\left[1+\frac{\phi^{2}}{4 E(\phi-E)} \sinh ^{2}\left(\frac{a \sqrt{2 m(\phi-E)}}{\hbar}\right)\right]^{-1},
$$

where $m$ is the particle mass and $a$ is the width of the box-shaped barrier. For small $\tilde{t}$ this expression can be approximated by:

$$
\tilde{t} \approx \frac{16 E(\phi-E)}{\phi^{2}} \exp \left(-2 a \frac{\sqrt{2 m(\phi-E)}}{\hbar}\right) .
$$

The prefactor is only dependent on the particle energy and barrier height, not on the barrier width. Furthermore, it smoothly scales with about $\frac{16 E}{\phi}$. In experimental studies, the prefactor is often omitted. One example is the Wentzel-Kramers-Brillouin (WKB) approximation used to approximate arbitrary shaped barriers with an integral over $\delta$-like box-shaped potentials:

$$
\tilde{t} \approx \exp \left(-2 \int_{\text {pot. }} \frac{\sqrt{2 m(\phi(x)-E)}}{\hbar} \mathrm{d} x\right),
$$

where $x$ is the position within the barrier and the integral extends over the width of the potential. $\phi(x)$ can be an arbitrary integrable positive function greater than $E$ describing the shape of the barrier. These approximations are discussed in quantum mechanics text books, such as Schwabl [104 and Fließbach 105.

One typical example of tunneling is the emission of cold electrons. The current is described by the Fowler-Nordheim formalism based on WKB approximation [106]:

$$
J=\alpha F^{2} \exp \left(-\frac{\beta}{F}\right)
$$

where $\alpha=1.5413 \cdot 10^{-6} \frac{m}{\phi m^{*}}$ and $\beta=6.828 \cdot 10^{7} \sqrt{\frac{\phi^{3} m^{*}}{m}} . m^{*}$ is the effective mass in the dielectric material. 


\subsection{Conductivity in amorphous materials}

Amorphous materials are usually non-metal ${ }^{8}$ and have a Fermi-energy inside a band gap as illustrated in figure 2.7. Hence, the most obvious conduction method would be the thermal activation of electrons into the conduction band according to equation 2.18. However, most band gaps are of the order of several electronvolt making thermal activation from the valence band unlikely at room temperature $\left(k_{\mathrm{B}} T=0.025 \mathrm{eV}\right)$. The irregular amorphous structure results in randomly altered energy levels of individual states compared to an ordered lattice. A significant number of states are shifted into the band gap forming band tails. In addition, the defect-rich nature of the amorphous material causes the formation of states deep inside the band gap. The defect- and tail states are limited in their mobility (localized), because no direct neighbors are necessarily available. Hence, amorphous materials have localized states within their band gaps as illustrated in figure 2.7 and figure 2.10. These localized states are filled up to the Fermi energy. At finite temperatures, some electrons can be thermally exited up to the conduction band from defects close to the Fermi energy, causing a current. In addition, thermal fluctuations allow electrons to migrate to nearby localized states (thermally assisted hopping). Furthermore, electrons can move by tunneling to neighboring states at the same energy level.

The development of modern conduction models for amorphous materials has begun in the 1940s. One early and effective model by Frenkel and Poole will be discussed later, this model describes thermal activation of trapped states into the conduction band and models the field dependency as well. Besides assuming a band-structure, this model is a completely classical model. A hopping model, which is based on a combination of thermal activation and tunneling was created in the late 1960s by Mott and is considered a semiclassical mode 9 .

All models and extension created before the 1980s could not use modern computer simulation and had to be solved analytically. The random nature of amorphous materials and the resulting complex band structure and potential landscape of charge carriers have to be approximated by averages and simplified distributions, which leads to sometimes questionable predictions. Modern approaches use Monte-Carlo simulations or molecular dynamic calculations to check the analytical approximations. Here, complicated barrier geometries, the true randomness of the structure, and the full quantum mechanical dependencies can be implemented. However, the results show that the classical and semiclassical models are still suitable to model the current transport in many applications. The microscopic interpretation of the analytical models should be done with care, since not all effects are accounted for correctly. In this work the semiclassical approaches by Mott and Frenkel-Poole are applied to interpret the electrical currents measured in ta-C films.

\footnotetext{
${ }^{8}$ metallic glasses shall be excluded from discussion here.

${ }^{9}$ electrons are treated as classical particles but quantum mechanical tunneling is included
} 


\subsubsection{Frenkel-Poole model}

The model for a conduction mechanism in amorphous materials named after Frenkel and Poole was published in 1938 by Frenkel [107]. The assumption is the presence of occupied localized states below the conduction band. By thermal activation, a trapped electron can be exited into the conduction band, where it can move freely until it is trapped again in a localized state below the conduction band. In parallel, thermal activation from the valence band is possible. However, the activation energy of valence-band electrons is much higher than the energy required for the trapped electrons. If a sufficient amount of localized states is available, the thermal activation from traps will be dominant. The localization potential in the Frenkel-Poole model (FP) is assumed to have $\frac{1}{x}$-shape (coulomb potential). The reduction of the barrier height at finite fields is $\beta_{\mathrm{FP}} F$, where $\beta_{\mathrm{FP}}$ is the Frenkel-Poole factor which can be calculated from electrostatics to be:

$$
\beta_{\mathrm{fp}}=\sqrt{\frac{q}{\pi \epsilon \epsilon_{0}}}
$$

with $\epsilon_{0} \approx 8.854 \times 10^{-12} \mathrm{Fm}^{-1}$ the vacuum permittivity, $\epsilon$ the relative permittivity (dielectric constant) and $q$ the charge (in case of electrons $q=e$ ). Assuming all traps are on the same energy level and using equation 2.18 leads to the thermal- and field-assisted hopping:

$$
J=\mu n_{\text {trap }} \exp \left(-\frac{\phi-\beta_{\mathrm{FP}} \sqrt{F}}{k_{\mathrm{B}} T}\right) F,
$$

where $\phi$ is the energy difference between the conduction band and the trap-level, and $n_{\text {trap }}$ the density of localized states. Combining equation 2.27 and equation 2.28 and combining all pre-exponential variables to one system dependent $\sigma_{\text {fp }}$ leads to the common representation of the Frenkel-Poole equation:

$$
J=\sigma_{\mathrm{fp}} F \cdot \exp \left[\frac{1}{k_{\mathrm{B}} T} \cdot\left(-q \phi_{\mathrm{fp}}+\sqrt{\frac{q^{3} F}{\pi \epsilon \epsilon_{0}}}\right)\right] .
$$

The FP model current transport mechanism is illustrated in figure 2.9(a) by the red dashed line $E_{2}$. The reduction of the potential is visualized by the green arrow marked with $\beta_{\mathrm{fp}} F^{1 / 2}$, where $F$ is the applied field. The electron is exited into the conduction-band and can move freely until it is caught by the next localized trap. The figure shows two alternative paths; these will be discussed while regarding the extension of the FP model. Since the barrier reduction is field-dependent, the amount of activated electrons, and therefore the conductivity will increase with field strength. This correlation leads to bend $I$ - $V$-characteristics with an $\exp \sqrt{F}$ dependence of the current on the field. 


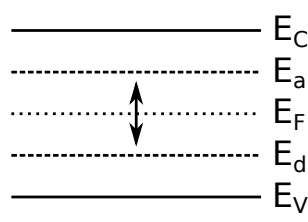

Figure 2.8: Band schematics with two localized state levels acting as donor and acceptor. The arrow indicates the activation for the FP between two levels of localized states as proposed by Simmons 108

One obvious simplification of the FP model is the assumption of an average energy level of the traps, which is most likely incorrect. This problem could be corrected by using multiple FP functions. However, this approach is only practical, if the DOS is known with sufficient precision (which is rarely the case), fitting it usually leads to an overdetermined system. The effect of assuming only one energy level $(\phi)$ in case of several different states results in unphysical parameter values (in particular for $\epsilon$ ) and in a wrong temperature dependence of the parameters, indicating that the model is not correct.

A second effect is referred to as barrier saturation (exhaustion). This effect is observed if the field and thermal excitations are large enough so $\beta_{\mathrm{fp}} \sqrt{F}$ is in the order of $q \phi_{\mathrm{fp}}$, which is often the case for very shallow states and always occurs at sufficiently high fields and temperatures. If this saturation is reached, all available sites contribute to current transport and a higher field will no longer increase their number. Instead of $\sigma(F) \propto \exp \sqrt{F}$ a more ohmic behavior is observed. The decreasing derivative of $I(F)$ in case of saturation reduces the value of the fitted $\beta_{\mathrm{fp}}$ leading to a seemingly higher $\epsilon$.

Furthermore, an inhomogeneity of the amorphous medium can lead to an apparently too high $\epsilon$. If the density of states fluctuates along the path of the current, the local field will not be constant. The FP effect will be most dominant where the field is large. However, at these positions the effective field is underestimated assuming a homogeneous sample leading to an apparently too small $\beta_{\mathrm{fp}}$.

The FP model suggests that most active localized states are close to the Fermi energy since the thermal excitation of these electrons to the conduction band has the highest probability and decreases very fast towards deeper energy levels. In this case, $\phi$ can be interpreted as the distance of the $E_{\mathrm{F}}$ to the mobility edge of the conduction band or valence band.Simmons [108] argues that $\phi$ should not be interpreted in this sense per se. He describes a FP mechanism solely utilizing localized states depicted in figure 2.8. In his opinion, a more or less symmetric distance of donor sites $E_{\mathrm{d}}$ below $E_{\mathrm{F}}$ and acceptor sites $E_{\mathrm{a}}$ above the Fermi energy is very likely. In this case, the measured activation is equivalent to $E_{\mathrm{a}}-E_{\mathrm{F}}=\frac{1}{2}\left(E_{\mathrm{a}}-E_{\mathrm{d}}\right)$. Interpreting this value falsely as the distance of the Fermi energy to the mobility edge leads to two wrong conclusions. First, no true knowledge of the position of the Fermi energy is obtained. Second, the barrier reduced by the field is $2 \phi$ in the context of equation 2.28, because the actual potential depth is $E_{\mathrm{a}}-E_{\mathrm{d}}$. Fitting $\beta_{\mathrm{fp}}$ assuming the experimental activation to be equivalent to $\phi$ results in apparently four times larger value for $\epsilon$. 
To derive the correct function for Simmons interpretation shown in figure 2.8, first from equation 2.14 and equation 2.13 the conductivity at zero bias $\sigma_{0}$ is calculated:

$$
\sigma_{0} \propto \exp \left(\frac{E_{\mathrm{a}}-E_{\mathrm{d}}}{2 k_{\mathrm{B}} T}\right)
$$

assuming $E_{\mathrm{F}}=\frac{1}{2} E_{\mathrm{a}}+E_{\mathrm{d}}$. The barrier is $\phi$ is equivalent to $E_{\mathrm{a}}-E_{\mathrm{d}}$. Equation 2.29 must be rewritten as

$$
J=\sigma_{\mathrm{fp}} F \cdot \exp \left[\frac{1}{k_{\mathrm{B}} T} \cdot\left(-q \phi_{\mathrm{fp}}+\sqrt{\frac{q^{3} F}{4 \pi \epsilon \epsilon_{0}}}\right)\right] .
$$

Simmons points out, that in this formulation of the FP model the field dependence $\beta$ is equivalent to $\beta$ of the Schottky emission. Therefore, it is not possible to distinguish Schottky emission (equation 2.22 and FP by the fitted value of $\epsilon$. The expected difference of a factor four is not necessarily observed.

Finally, the FP model neglects any current transport due to tunneling. However, as shown in figure 2.9 $(a)$ the electron can also tunnel into the conduction band if a sufficiently high field is applied. The WKB-approximation allows the representation of a tunnel probability through an arbitrary barrier by an exponential function. The magnitude of the integral depends on the barrier height and width as seen in equation 2.25. For ease of presentation, the integral is written as $r(x)$. The tunnel probability can now be expressed as

$$
\tilde{t} \propto \exp [-2 r(x)]
$$

The migration of the electrons is possible by a combined process where the electron is first elevated thermally close to the conduction band edge and then tunnels into the band without going over the barrier. The probability of this process is the product of the thermal activation and the tunnel probability. An example of this transport is shown in figure 2.9(a) by the purple level $E_{1}$. The tunneling probability is practically temperature independent 106. The tunneling process becomes increasingly more important at low temperatures. The purple and blue path in figure 2.9(a) are dominant at low temperatures. A more thorough description of tunneling will be given in the next section chapter 2.5.2.

The FP hopping length and resulting conductivity are affected by magnitude and change of the DOS as indicated by theoretical calculations by Bleibaum [109]. As thermally activated FP saturates, the dependency on tunneling (distances and number of hopping sites) becomes more important, similar to the calculations of Grünewald 110,111]. The assumption of a constant level of DOS induces a shorter localization length $\alpha^{-1}$ leading to larger barriers as described later in variable range hopping.

El-Samanoudy et al. analyzed the $I$ - $V$-characteristics of $\mathrm{Ge}_{25} \mathrm{Bi}_{\mathrm{x}} \mathrm{Sb}_{15-\mathrm{x}} \mathrm{S}_{60}$, using different extensions of the FP model $[112]$. They suggested two models, one by 
Hall [113 assuming two activation energies. El-Samanoudy uses this model to explain low temperature deviations of $\epsilon$ obtained with the original FP model. The other by Hill [114] where multiple contributions of thermally activated tunneling are included. Again it is derived, that the conduction channel of pure FP cannot be expected to be observed; additional contributions are required [114]. In other words, FP is most likely accompanied by tunneling.

The determination of $\epsilon$ suffers from these uncertainties and simplifications of the original model. Analysis of the limits and extensions of the FP model as given for by Hill [114], Hall [113], and Jonscher [115] basically suggest the addition of transport channels in parallel to the original FP. An approach to assume a three-dimensional potential topography was given by Hartke [116. The conclusion of the authors supports the effectiveness of the FP model to describe conduction in amorphous thin films. However, for several reasons, the original model is not effective in predicting the correct $\epsilon$ values, essentially for the reasons given above. Applying the correct extension to FP requires an extensive knowledge of DOS and other film properties.

The model is suitable to fit the current behavior of defect-rich amorphous semiconductors at high temperatures where thermal activation dominates. The model accounts numerically for non-ohmic $I-V$-characteristics. Instead of fitting $\epsilon$, the deviation from the expected value may hint an alternative conduction path as proposed by Simmons, Hill or Hall. It is not reliabale to use the FP model to determine the position of $E_{\mathrm{F}}$ with respect to the mobility edge, neither should an $\epsilon$ be extracted from the fits.

\subsubsection{Variable Range Hopping}

The FP model describes thermally activated electron transport where electrons from localized states are lifted into the conduction band. An applied electrical field lowers the barrier for the transport, thus the current is field-dependent. In high-field situations, direct tunneling into the conduction band or a combination of thermal activation and tunneling as shown (figure 2.9(a)) is possible. Now, considering the situation at zero-bias, the FP model converges to simple thermal activation without barrier lowering. Tunneling into the conduction band is impossible. However, tunneling (hopping) to neighboring states with the same energy level is possible as sketched by the blue dotted path in figure 2.9(b). The ease of tunneling increases with higher thermal activation (dashed-doted purple path). At even higher temperatures, the electron may jump over the barrier solely by thermal activation (red-dashed path). As temperature decreases, longer tunneling paths become relevant. This temperature dependent average tunneling range leads to the Variable Range Hopping (VRH) model first proposed by Mott [117]. Its $\sigma \propto \exp T^{0.25}$ dependency is successfully fitted to systems as amorphous $\mathrm{SiO}_{\mathrm{x}}$ [118].

In the following, the mathematical description of the mechanisms sketched in figure 2.9(b) will be reviewed. The energies and locations of states in amorphous solid 


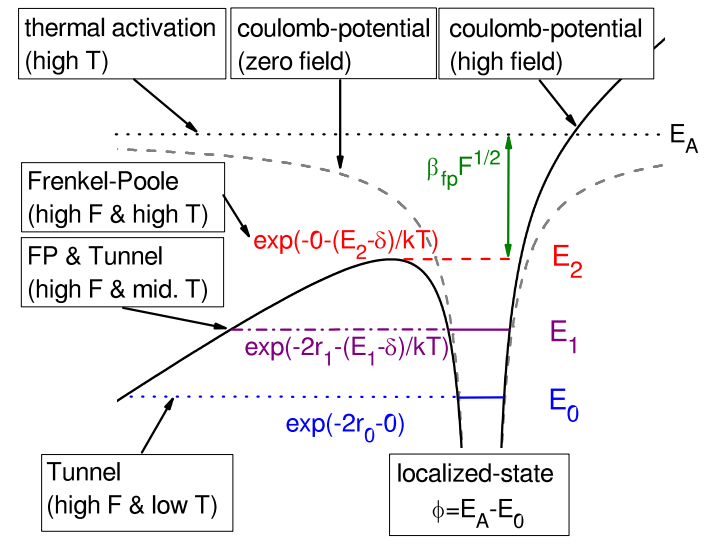

(a) Schematics of FP

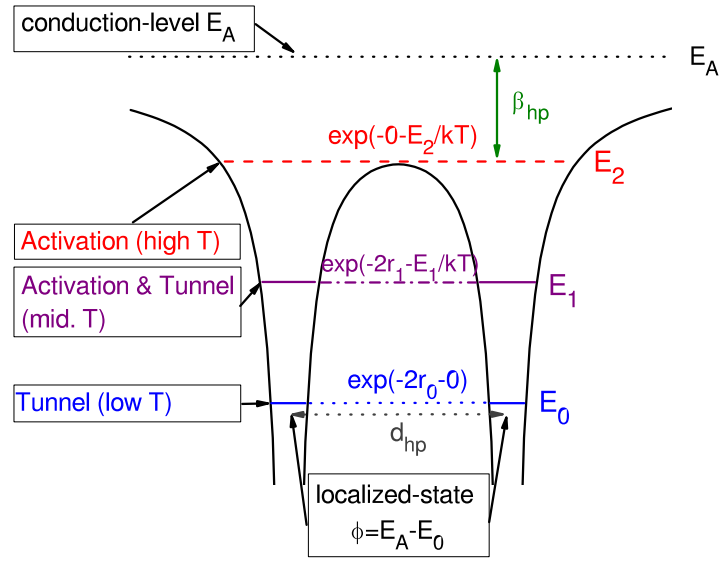

(b) Schematics of hopping

Figure 2.9: Schematics of the transport mechanisms from a Coulomb trapped localized state $E_{0}$ into the conduction band in high field case (a). The thermal activation $\left(E_{2}=E_{\mathrm{A}}-E_{0}\right)$ is reduced by the FP effect by $\delta=\beta_{\mathrm{fp}} \sqrt{F}$, the excitation probability is proportional to exp $\frac{E_{2}-\delta}{k_{\mathrm{B}} T}$. At $E_{1}$ and $E_{0}$ two other path are shown. The thermally excited tunneling through $r_{1}$ and the pure tunneling without any thermal excitation at $r_{0}$. In (b) a low field example of three possible hopes from one to another localized state are shown. Hopping is possible by pure thermal excitation, excitation and tunneling or only tunneling. Again, each path is probability given by the exponential functions. Reducing the distance between two sites lowers the barrier height by $\beta_{\mathrm{hp}}=\frac{q^{2}}{4 \pi \epsilon \epsilon_{0} d_{\mathrm{hp}}}$.

are randomly distributed. This description of the non-periodic nature of the environment is the most critical point of formulating an analytical model. In fact, the simplifications made by Mott in this respect are the most criticized part of the theory. However, other authors applying different mathematical and analytical approaches propose the same temperature dependence. Some notable works were done by Apsley and Hughes [119, 120] and Bourbie [121,122]. Early on, percolation approaches were applied to VRH, as for example, by Pike and Seager [123, 124] as well. The results are similar to the analytical results published by Mott. I will focus on the original approach to VRH including some arguments especially by Apsley and Hughes.

Taking the Fermi-Dirac distribution into account (equation 2.14), the hopping current from site $i$ to $j$ is

$$
I_{\mathrm{ij}}=f_{\mathrm{i}}\left(E_{\mathrm{i}}\right)\left[1-f_{\mathrm{j}}\left(E_{\mathrm{j}}\right)\right] \omega_{\mathrm{ij}}-f_{\mathrm{j}}\left(E_{\mathrm{j}}\right)\left[1-f_{\mathrm{i}}\left(E_{\mathrm{i}}\right)\right] \omega_{\mathrm{ji}},
$$

where $\omega_{\mathrm{ij}}=\nu p_{\mathrm{ij}}$ is the transition rate which is the product of the trial frequency $\nu$ and the hopping probability $p_{\mathrm{ij}}$. The trial frequency is physically related to the phonon frequency [125]. The tunnel rate is estimated with WKB-approximation and is expressed as

$$
\tilde{p}_{\mathrm{ij}}=\exp \left(-2 \alpha\left|R_{\mathrm{ij}}\right|\right)
$$


where $R$ is the distance between the hopping sites and $\alpha^{-1}$ the localization length. In one dimension, $\alpha^{-1}$ corresponds to the attenuation length for a hydrogen-like localized wave-function into the barrier depending on the barrier height. It is in the order of the Bohr-radius. In higher dimensions, the system becomes mathematically more complicated and the parameter $\alpha$ depends upon the three dimensional potential landscape as all possible tunneling paths through space have to be considered. Accounting for the Fermi-Dirac-statistics and the activation needed for "up-hill tunneling" as well as assuming $\left|E_{\mathrm{i}}-E_{\mathrm{j}}\right|>k_{\mathrm{B}} T$ and low electric fields across the involved hopping sites $\left(q F R_{\mathrm{ij}}<k_{\mathrm{B}} T\right)$ leads to the Miller and Abrahams [126] conduction:

$$
I_{\mathrm{ij}} \propto \nu \exp \left(-2 \alpha\left|R_{\mathrm{ij}}\right|+\frac{\left|E_{\mathrm{i}}-E_{\mathrm{F}}\right|+\left|E_{\mathrm{j}}-E_{\mathrm{F}}\right|+\left|E_{\mathrm{i}}-E_{\mathrm{j}}\right|}{2 k_{\mathrm{B}} T}\right) .
$$

In case of high fields, the Fermi energy cannot be assumed constant at both hopping sites and equation 2.35 is extended to:

$$
I_{\mathrm{ij}} \propto \nu \exp \left(-2 \alpha\left|R_{\mathrm{ij}}\right|+\frac{\left|E_{\mathrm{i}}-E_{\mathrm{F}}\right|+\left|E_{\mathrm{j}}-E_{\mathrm{F}}\right|+\left|E_{\mathrm{i}}-E_{\mathrm{j}}\right|-\left|E_{\mathrm{Fi}}-E_{\mathrm{Fj}}\right|}{2 k_{\mathrm{B}} T}\right),
$$

where $E_{\mathrm{Fi}}$ is the Fermi energy at hopping site $i$. Mott simplified equation 2.35further taking out the effect of the Fermi-Dirac statistics assuming all relevant hopping states are within $k_{\mathrm{B}} T$ of the Fermi energy. This assumption is questionable and a point of criticism. However, it has been shown by Apsley and Hughes [119, 120 that this simplification does not significantly affect the behavior of the overall model (Temperature dependence). Mott's approximation leaves a very convenient form for the hopping probability:

$$
p_{\mathrm{ij}} \approx\left\{\begin{array}{ll}
\exp \left(-2 \alpha R_{\mathrm{ij}}\right) & E_{\mathrm{j}} \leq E_{\mathrm{i}} \\
\exp \left(-2 \alpha R_{\mathrm{ij}}-\frac{E_{\mathrm{j}}-E_{\mathrm{i}}}{k_{\mathrm{B}} T}\right) & E_{\mathrm{j}}>E_{\mathrm{i}}
\end{array} .\right.
$$

For a more convenient representation, the spacial and energetic distances of the two hopping sites are combined into one $\mathcal{R}$ as done for example by Apsley and Hughes:

$$
\mathcal{R}_{\mathrm{ij}}=-\ln \left(p_{\mathrm{ij}}\right)
$$

The hopping probability is largest if the four dimensional distance in hoping-space $\mathcal{R}$ is smallest. If the DOS is very high $\left(\alpha d_{0} \ll 1^{10}\right)$, hopping will be most dominant to the nearest neighbor in space. The conductivity of this nearest-neighbor hopping depends on a small thermal activation to reach the destination site (equation 2.18). An example for nearest-neighbor-hopping would be the electron transport in donor levels (high DOS density in small energy band) of a crystalline semiconductors 127,128]. If

\footnotetext{
${ }^{10}$ where $d_{0}$ is the average distance to the nearest neighbors
} 
the density of localized states is smaller $\left(\alpha d_{0} \gtrsim 1\right)$, the tunneling part of the hopping probability (second part of equation 2.37) becomes more important leading to VRH.

The hopping probability between two sites in the four dimensional hopping space depends exponentially on spacial and energetic distance. Therefore, the dominance of few favorable paths is expected. From all neighbor sites $\mathcal{R}_{\mathrm{ij}}$ the minimum is selected and denoted as $\mathcal{R}_{\mathrm{i} \mathrm{nn}}$, the nearest neighbor site. Furthermore, an average distance for all relevant hopping sites is assumed $\overline{\mathcal{R}}$ and the resulting electron transport is proportional to the product of the probability $p$ of each step $k$ up to the final number of hops $m$ through the solid which is assumed to be constant $(\bar{p})$ :

$$
\sigma \propto\left(\prod_{k}^{m} p_{k}\right)^{1 / m} \stackrel{p_{k}=\bar{p}}{=} \bar{p}=\exp (-\overline{\mathcal{R}})
$$

The mathematical approximation of $\overline{\mathcal{R}}$ is critical debated in literature $119,120,125$, 129 131. Motts method is shown to some extend in his book [125]. Alternative approaches are carried out by Apsley and Hughes [119, 120], for example, leading to the same over temperature dependence. Furthermore, the DOS is assumed to be constant over the regime of active hopping sites (independent of energy). This assumption has been debated for example by Hamilton [130 and Pollak 131] as well as Apsley and Hughes in their publications cited above. In any case, the proportionality of equation 2.39 is unchanged. The prefactor itself varies between different mathematical treatments of the problem as it is discussed by the above publications. Mott suggested for optimum hopping range (minimizing the distance within the four dimensional hopping space)

$$
\overline{\mathcal{R}}=2 \alpha \frac{3^{1 / 4}}{\left(2 \pi \alpha N\left(E_{\mathrm{F}}\right) k_{\mathrm{B}} T\right)^{1 / 4}},
$$

which gives the famous temperature dependence:

$$
J=F \sigma_{\mathrm{vrh}} \exp \left(\frac{-B}{T^{1 / 4}}\right) .
$$

The variables are equivalent to:

$$
B=2\left(\frac{3 \alpha^{3}}{2 \pi k_{\mathrm{B}} N\left(E_{\mathrm{F}}\right)}\right)^{1 / 4}=2\left(\frac{3}{2 \pi}\right)^{1 / 4}\left(\frac{\alpha^{3}}{k_{\mathrm{B}} N\left(E_{\mathrm{F}}\right)}\right)^{1 / 4} \approx 1.66\left(\frac{\alpha^{3}}{k_{\mathrm{B}} N\left(E_{\mathrm{F}}\right)}\right)^{1 / 4}
$$

and

$$
\sigma_{\mathrm{vrh}}=\nu e^{2} N\left(E_{\mathrm{F}}\right)\left(\frac{\overline{\mathcal{R}}}{2 \alpha}\right)^{2} .
$$

Mott does not use the four-dimensional notation of $\bar{R}$, he uses $\bar{R}=\left(\frac{\overline{\mathcal{R}}}{2 \alpha}\right)$ instead. According to equation 2.40 the prefactor has a $\frac{1}{T^{2}}$ dependence assuming constant 
phonon frequency and DOS constant at the Fermi-energy. Equation 2.41 is one of the possible representation of the generalized form of equation 2.39 deriving the prefactor according to Mott 125. As described above, other authors using different mathematical approaches or percolation theory present similar results showing the same temperature dependence. A further collection of works is summarized by Mott in his book [125].

The above formula is true only for small fields. In case of larger fields, the correction as already shown in equation 2.36 for the MA conduction has to be carried through the process of minimizing the hopping ranges $\mathcal{R}$. Different approximations lead to different field dependencies, while the overall temperature dependence is preserved. One example of moderate-field correction of Mott's VRH is the model of Pollak and Riess 132. Mott's equation (equation 2.41) is extended to be:

$$
J=F \sigma_{\mathrm{vrh}} \exp \left(\frac{-B}{T^{1 / 4}}+\frac{q F \tilde{\gamma}_{\mathrm{p}} \bar{R}}{k_{\mathrm{B}} T}\right),
$$

where $\tilde{\gamma}_{\mathrm{p}}=0.17$ is a proportional factor. $\bar{R}$ is the low-field average hopping range $\bar{R}=\left(\frac{\overline{\mathcal{R}}}{2 \alpha}\right)$. This approximation is valid for fields and temperatures not exceeding $\frac{q F}{2 \alpha k_{\mathrm{B}} T}<1$.

\subsubsection{Conductivity of ta-C}

The key to the electronic conduction behavior of ta-C is its bond structure. In the $\mathrm{sp}^{3}$-configuration, carbon has four $\mathrm{sp}^{3}$ orbitals forming strong $\sigma$ bonds to the neighboring atoms. In the $\mathrm{sp}^{2}$ configuration, three $\mathrm{sp}^{2}$ orbitals form planar $\sigma$ bonds; the fourth orbital is $\pi$ bond. The electrons in $\sigma$-bonds fill states in the valence band, whereas the empty $\sigma^{*}$ sites are located in the conduction band. The $\pi$ states and $\pi^{*}$ states are distributed analogously. The overall distribution of states with respect to the Fermi energy is illustrated in figure 2.10. Diamond, only bonded by $\sigma$ bonds in $\mathrm{sp}^{3}$-configuration, is an insulator with a wide band gap (large separation of $\sigma$ bands). Graphite, on the other hand, is a (metallic) conductor, where the states, forming the $\pi$ and $\pi^{*}$ bands, touch at the Fermi-energy. The band structure of a-C is formed by both $\sigma$ and $\pi$ electrons. In ta-C, where the $\mathrm{sp}^{3} / \mathrm{sp}^{2}$ ratio is high, the band gap is preserved. Furthermore, the high defect density introduces a large amount of defect states into this band gap as illustrated in figure 2.10. Both bands show exponential tails into the gap, these states are localized, as well as, the defect states. The relative energy positions of the valence band edge $\left(E_{\mathrm{V}}\right)$ and the conduction band $\left(E_{\mathrm{C}}\right)$ are shown with respect to the Fermi energy. The values for DOS as well as the energy scale are rough estimations based on ref. [37].

In a-C, the Fermi energy is estimated to be about mid-gap. Some authors proposed a Fermi-energy closer to the valence-band in case of ta- $\mathrm{C}$ 133, 134. The value published is about $0.3 \mathrm{eV}$ [134 137], which is actually small. In case of a band gap 
in the order of $2 \mathrm{eV}$ a pronounced p-type behavior should be visible, which is not the case 34,35, 138 (see also chapter 2.5.4). Most likely, the measured thermal activation was misinterpreted to resemble the distance of the Fermi energy to the valence band (compare Simmons argument in chapter 2.5.1p). Electrical characterization of MSIBD deposited ta-C shows no sign of either n- or p-type conduction 34, 35, 103.

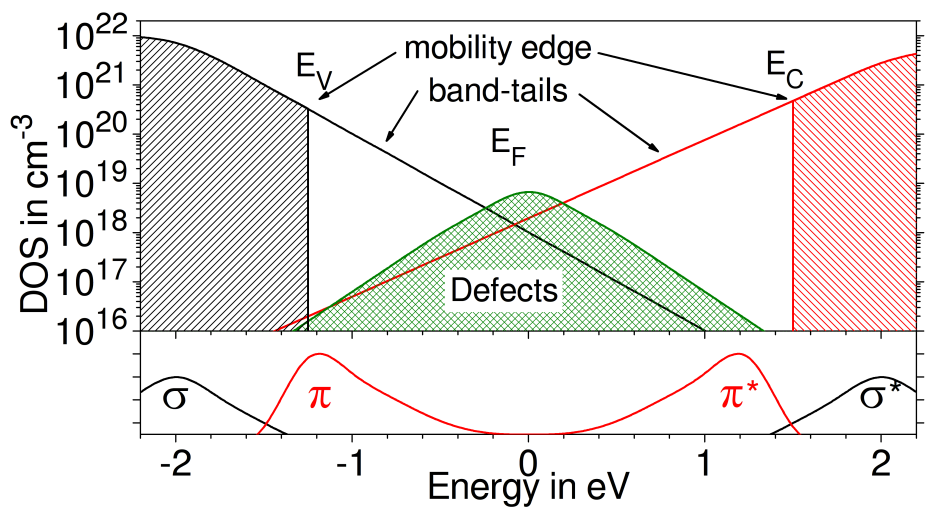

Figure 2.10: Schematic graph of the band structure of ta-C showing the continuous bands, the localized tales of the bands and the defect states. Underneath the density of states the bond types making up the states with respective energy are sketched. All numbers are estimates and should present a qualitative overview as estimated by Robertson 37.

The electron distribution shown in figure 2.10 does not indicate single conduction mechanism, instead multiple possibilities are plausible. Four obvious channels for $\mathrm{a}-\mathrm{C}$ and ta-C conduction are:

- Thermal activation of localized states to the mobility edge (unlikely at moderate temperatures as the band gap is estimated to be in the order of $2 \mathrm{eV}$ or more and a thermal activation of $0.3 \mathrm{eV}$ has been observed.)

- Nearest neighbor hopping in localized states close to the Fermi-energy

- FP conduction at high fields and temperatures - most likely in the manner proposed by Simmons

- Variable range hopping, especially at lower temperatures and higher defect densities (tracks and impurities)

Other authors also suggest the coexistence of multiple conduction mechanisms, especially hopping between localized states [135, 139].

The band structure of a- $\mathrm{C}$ and ta-C depends on the ratio of the sp-hybridization determining the amount of $\sigma$ and $\pi$ bonds. In figure 2.11 140,141], the DOS are shown for different $\mathrm{sp}^{3}$ contents. In all cases $E_{\mathrm{F}}$ is about midway of the band gap and the defect states are distributed more or less symmetrically to the Fermi energy supporting the argument of no natural p-type behavior. The figure shows the decreasing 
number of $\pi$ states as the $\mathrm{sp}^{3}$ ratio increases. Furthermore, the strong localization of states around the band gap is visible (increasing defect density).

\subsubsection{Impurity conduction in ta-C}

A decade ago, substantial research was devoted to find dopants for a-C and ta-C. Probably driven by this quest arose the assumption of the natural p-type behavior of ta-C based on its thermal activation [133, 134]. However, up to today, no successful doping has been found. In crystalline silicon doping is easily achieved with boron and aluminum (acceptors) and nitrogen and phosphorous (donors). The same dopants would be promising for carbon. Doping diamond is much more difficult than silicon, although both have the same structure. Besides the problems raised by incorporating dopants in diamond [142], it is hard to place shallow levels into the wide band gap. Nitrogen, for example, creates deep donor levels $1.7 \mathrm{eV}$ inside the band gap [37]. While solubility of dopants is a major problem in diamond [142], it is easy in aC. The main problem of doping amorphous semiconductors lies in the freedom of atoms to exert their chemically preferred valence structure [143 forming defect-like non-doping sites with lower energies. In a-Si doping is still possible using higher concentrations [144 compared to its crystalline counterpart. Up to now, doping has failed in ta-C. Experiments trying to form p-n junctions composed of doped ta-C have not been successful [35]. Instead, theoretical calculations predict no doping effect 145. One reason is the high DOS in the band gap in pure ta-C . Doping requires the movement of the Fermi level closer to a mobility edge becoming increasingly more difficult with a high density of defect states pining the Fermi level [146]. In a-Si, the effect is observed as well, but can be overcome with high doping concentrations [144] as the defect density [147] is smaller compared to ta-C [148]. In addition, in a-C, N is bonded to structures as aromatic rings with $\mathrm{sp}^{2}$ - instead of $\mathrm{sp}^{3}$-hybridization. In the end, $\mathrm{N}$ mainly increases the defect density and reduces the $\mathrm{sp}^{3} / \mathrm{sp}^{2}$ ratio leading to a higher conductivity [149,150], but no doping [34,35]. Similarly, Boron should act as a p-dopant 151. However, for essentially the same reason, no doping effect has been shown for B [35]. Again, only the conductivity increases [25, 35].

Copper is known to have a low solubility in ta-C $(<0.01$ at.\% [152]) forming clusters embedded in the ta-C matrix. Gerhards et al. [153] visualized these clusters in MSIBD-deposited films with $5 \% \mathrm{Cu}$ content ( $3 \mathrm{~nm}$ diameter). Unlike $\mathrm{N}$ and $\mathrm{B}, \mathrm{Cu}$ is almost inert in ta-C , a doping effect in terms of $\mathrm{n}$ - or p-doping was never expected. Instead, the $\mathrm{Cu}$ clusters increase the defect density and the effective surface of the carbon matrix. Cu surfaces are successfully used to grow epitaxial graphene 154,155 leading to the speculation that $\mathrm{Cu}$ surfaces increase the likelihood of $\mathrm{sp}^{2}$ bond carbon. Experimentally, the shift towards $\mathrm{sp}^{2}$ bonds in a-C films is observed at higher copper concentration (see results of XPS measurements chapter 44). Copper improves the conductivity of the ta-C films by increasing the DOS in the band gap. 


\subsubsection{Track conductivity in ta-C}

The irradiation of the ta-C locally increases the $\mathrm{sp}^{2}$ content as well as the defect density forming conductive ion tracks. In figure 2.11, which was taken from [140], the density of states, measured by participation ratio spectra, is shown for three different $\mathrm{sp}^{3}$ contents visualizing the variation of DOS with hybridization ratio. The number of $\pi$ states, which increase the conductivity, grows significantly with decreasing $\mathrm{sp}^{3}$ hybridization. Schwen et al. 23] estimated the $\mathrm{sp}^{2}$ ratio inside a track to be $60 \%$ as result of MD-simulations compared to $20 \%$ in the matrix surrounding it.

Similar to the increase in conductivity caused by impurities, the basic transport mechanism does not change. Instead, the DOS increases. Both effects can be combined, as experiments show, higher track conductivity in irradiated films containing impurities 24,25]. 


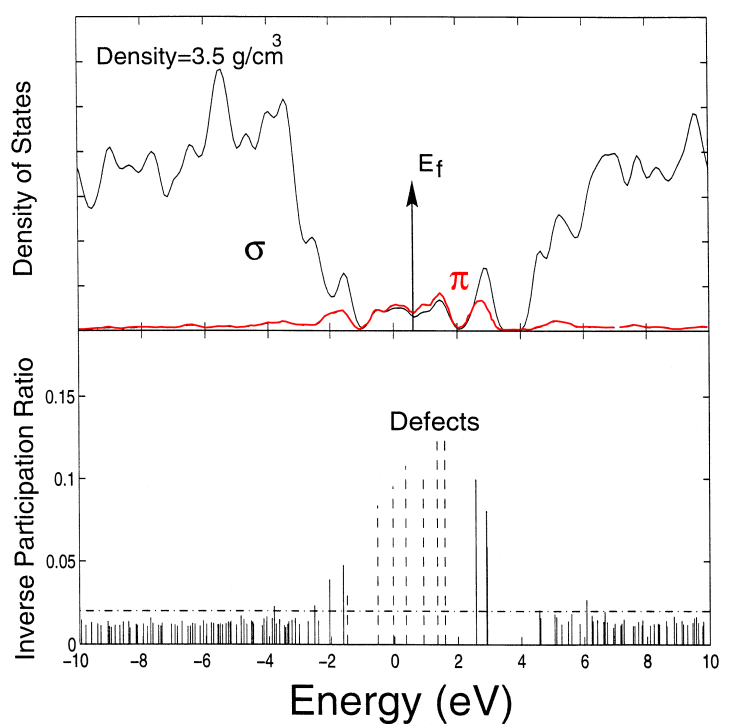

(a) $92 \% \mathrm{sp}^{3} \mathrm{a}-\mathrm{C}$

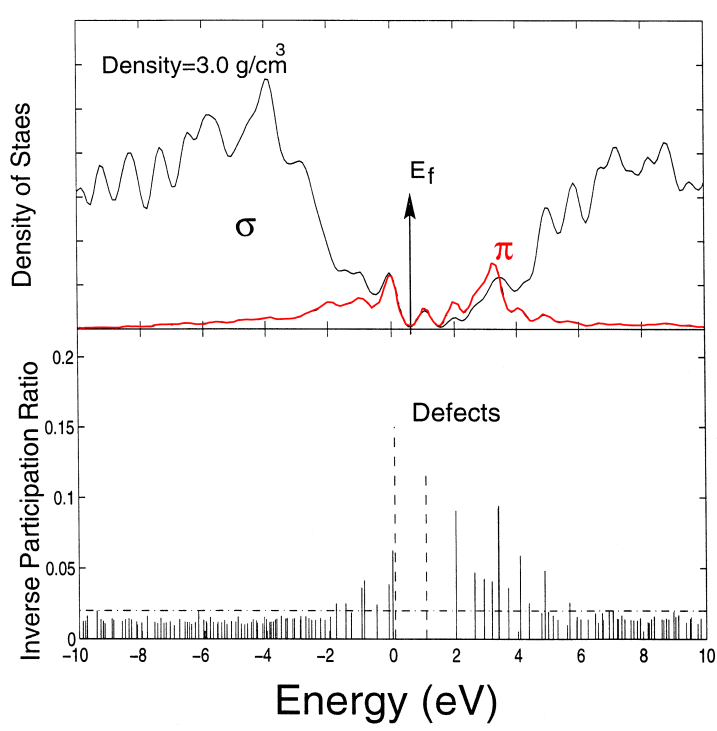

(b) $69 \% \mathrm{sp}^{3} \mathrm{a}-\mathrm{C}$

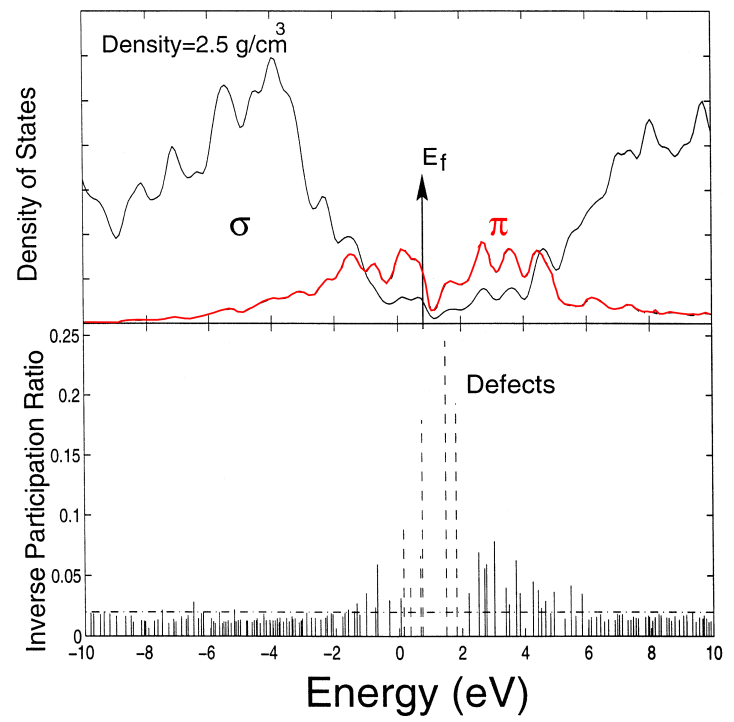

(c) $20 \% \mathrm{sp}^{3} \mathrm{a}-\mathrm{C}$

Figure 2.11: DOS and Inverse participation ratio spectra for different a-C networks with different hybridization ratios 140. The amount of $\pi$ states and defects within the band gap increase with reduced $\mathrm{sp}^{3}$. The reduced band gap and the increased DOS within decrease the resistivity of the $\mathrm{a}-\mathrm{C}$. 


\section{Experimental methods}

\subsection{Mass selected ion beam deposition}

Mass selected ion beam deposition (MSIBD) is a method of thin film coating by directing a filtered low energy ion beam onto a target. The desired ions are provided by the ion source. Depending on ion type, different techniques are implemented to ionize the atoms or molecules. One common type is the penning source, where a diluted gas around a hot filament is ignited to a plasma. This source works well, if the desired specimens are available in a gaseous form or are easily evaporated. Other techniques of ion generation include sputter sources and cathodic arcs. In any case, the ions are accelerated by an extraction potential. Ion optics are used to focus and sweep the ion beam onto the target. In case of mass selection, the beam passes a bending magnet, filtering a field depended $\frac{e}{m_{e}}$ ratio, thus allowing the selection of a specific isotope. Besides providing a highly purified beam of the selected ion specimen, the energy for the deposition can be controlled precisely ${ }^{1}$.

Samples analyzed in this work were synthesized by the MSIBD setup ADONIS (Anlage zur Deposition niederenergetischer Ionen) at the II. Institute of Physics of the University Göttingen. A schematics of the accelerator is shown in figure 3.1, it is routinely used for preparing ta-C BN and other films.

Both ta-C and BN films were deposited using a penning type ion source with multiple gas feeds and an oven to evaporate solids and liquids to be ionized. While a plasma is burning, the typical working pressure of the source is in the order of $1 \times 10^{-4}$ mbar to $1 \times 10^{-5}$ mbar and the typical extraction bias is $30 \mathrm{kV}$. The first electrostatic lens focuses the beam before entering the $90^{\circ}$ bending magnet. The beam direction can be corrected by the aid of the beam shift. The second electrostatic lens and the quadrupole lens are used to correct the beam profile in order to yield a quadratic and homogeneous beam profile. To filter out neutral particles (recombination with residual gas), the beam is bent by a small angle using the electrostatic deflector. Finally, the beam is wobbled by the beam sweep to yield a larger, more homogeneous irradiation spot and decelerated before hitting the target. Using a high extraction potential and decelerating the beam to the desired values has the advantage of higher extraction currents (Richardson's Law), and optical elements need no substantial adjustments upon deposition energy switching. The charge reaching the target is measured by an electronic current integrator. This information is fed to a PC and used to calculate the amount of deposited ions. The software can control

\footnotetext{
${ }^{1} \mathrm{~A}$ penning source uses a hot filament to generate ions with a thermal fluctuation of less than $2 \mathrm{eV}$.
} 


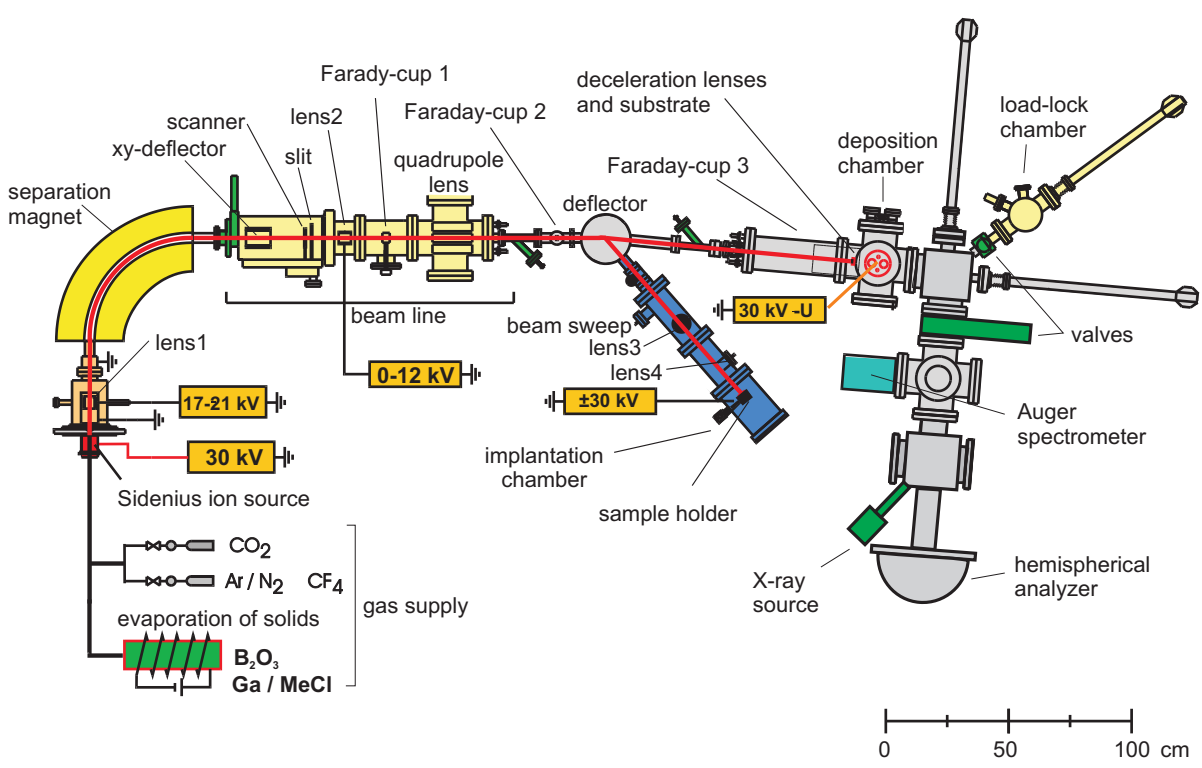

Figure 3.1: Illustration (adoption from 156) of the accelerator ADONIS used for preparing the ta-C films by mass selected ion beam deposition.

the deposition energy and charge as well as the magnetic field of the bending magnet (ion type).

It is possible to deposit two or more different ion types quasi simultaneously by switching the magnet between two "masses" every $10^{15}$ atoms (about one monolayer). Previous works, analyzing samples prepared by ADONIS, for example, by Zutz [157 or Gerhards et al. 153, 156] showed by transmission electron microscopy no correlation of the switching process and the sample composition. Brötzmann 103 performed structural analysis of BN films grown by MSIBD in ADONIS. The structure is not correlated to the rapid switching process either.

\subsection{1 ta-C fabrication}

First, the highly doped n-type 111-silicon substrates $\left(20 \mathrm{kS} \mathrm{m}^{-1}\right)$ were cleaned with acetone and 2-propanol. It is important to remove the final cleaning solvent by blowing it off the sample, if it would dry, non-evaporating dirt remains on the substrate surface. As substrates, highly doped n-type 111-silicon was used. After mounting the sample in the deposition chamber, the silicon surface was sputtered with a $1000 \mathrm{eV}$ ${ }^{40} \mathrm{Ar}^{+}$beam and a fluence of $5.5 \times 10^{16} \mathrm{~cm}^{-2}$. This sputter cleaning removes the native oxide layer of the silicon, microscopic dirt and amorphizes the surface improving the adhesiveness and contact of the ta-C film to the substrate. Immediately after this cleaning procedure, the actual carbon deposition was started. The source was fed with $\mathrm{CO}_{2}$ gas and ${ }^{12} \mathrm{C}^{+}$was deposited with $100 \mathrm{eV}$ onto the Si. Typical deposition rates are in the order of $10 \mathrm{~nm} \mathrm{~h}^{-1}$ at a current density of $15 \mu \mathrm{A} \mathrm{cm}{ }^{-2}$. 
The BN interlayers were prepared with an energy of $100 \mathrm{eV}$ as well. The $\mathrm{N}$ ions were provided by $\mathrm{N}_{2}$ gas, the $\mathrm{B}$ came from evaporating $\mathrm{B}_{2} \mathrm{O}_{3}$ in the oven. The magnet was used to switch the ion beam from $\mathrm{N}$ to $\mathrm{B}$ every $1 \times 10^{15}$ atoms. An in-depth analysis of the structural properties of $\mathrm{BN}$ prepared in this manner was performed by Brötzmann [103].

The $\mathrm{Cu}$ impurities were incorporated into the ta- $\mathrm{C}$ by switching between $\mathrm{C}$ and $\mathrm{Cu}$ atoms in different stoichiometry. The Copper was provided by evaporating $\mathrm{CuCl}_{2}$. Again, all deposition energies were set to $100 \mathrm{eV}$. Gerhards et al. proposed a model for metal incorporation in a-C [158]. No effect of the switching process was seen, instead a self-organization behavior forming multilayers was observed.

\subsection{Swift Heavy Ion Irradiation}

The majority of samples were irradiated at the GSI Helmholtzzentrum für Schwerionenforschung at the UNILAC ${ }^{2}$ using the beam of the UNILAC accelerator. In particular, a ${ }^{238} \mathrm{U}^{28+}$ beam with an energy of $11.6 \mathrm{MeV} \mathrm{u}^{-1}$ was used. The accelerator uses high frequency cavities to reach this energy, the beam is provided in bunches (packages), in case of the UNILAC the frequency is $50 \mathrm{~Hz}$. Each bunch has a length of about $3 \mathrm{~ms}$ to $10 \mathrm{~ms}$ followed by a few milliseconds pause. Typically, not all packages are used for one experiment, sometimes only three bunches per second are available for one particular experiment. The flux of a ${ }^{238} \mathrm{U}^{28+}$-beam is in the order of $1 \times 10^{8} \mathrm{~s}^{-1} \mathrm{~cm}^{-2}$ to $1 \times 10^{9} \mathrm{~s}^{-1} \mathrm{~cm}^{-2}$, a single bunch consists of about $5 \times 10^{7} \mathrm{~cm}^{-2}=$ $0.5 \mathrm{\mu m}^{-2}$. The probability of more than one ion per bunch hitting the same $\mu^{2}$ is small and therefore, it is safe to treat each hit as an individual independent event.

The final fluence was usually chosen between $1 \times 10^{9} \mathrm{~cm}^{-2}$ to $1 \times 10^{10} \mathrm{~cm}^{-2}$. In this range, 10 to 100 tracks are expected per $\mu^{2}$. Assuming a uniform pattern of the impinging coordinates at $1 \times 10^{10} \mathrm{~cm}^{-2}$, the average distance of two track centers is $151 \mathrm{~nm}$. In case of randomly distributed tracks with this fluence, $30.5 \%$ of the tracks have no neighbors within $100 \mathrm{~nm}, 69.4 \%$ have a free radius of at least $50 \mathrm{~nm}$, $93.8 \%$ have no neighbor within $20 \mathrm{~nm}$, and $98.4 \%$ have at least distance of $10 \mathrm{~nm}$ to the nearest track ${ }^{3}$. In other words, at $1 \times 10^{10} \mathrm{~cm}^{-2}, 98.4 \%$ of tracks are separated if the track radius does not exceed $5 \mathrm{~nm}$. Therefore, up to this fluence overlap of swift heavy ion tracks in ta-C is neglectable and tracks can be assumed to be separated structures. At $1 \times 10^{9} \mathrm{~cm}^{-2}, 99.8 \%$ of tracks do not overlap.

The beam energy of $11.6 \mathrm{MeV} \mathrm{u}^{-1}$ at the GSI is about a factor two above the Bragg peak with the largest $\frac{\mathrm{d} E}{\mathrm{~d} x}$ possible. Furthermore, the charge state $(28+$ in case of $\mathrm{U}$ ) is much lower than the equilibrium charge state reducing the energy loss further. Therefore, the ion energy is lowered to about $4 \mathrm{MeV} \mathrm{u}^{-1}$ to $5 \mathrm{MeV} \mathrm{u}^{-1}$ by an aluminum degrader foil. The required thickness of the foil is calculated by energy

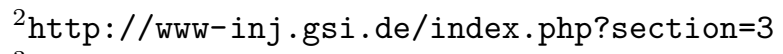

${ }^{3}$ All distances are measured center to center. 
loss simulation (SRIM) of the impinging ions. The degrader slows down the ion and changes the charge state according to the equilibrium distribution. The resulting stopping powers calculated with CasP are shown in table 2.1. The average stopping power of the ion impinging the ta-C is about $40 \mathrm{keV} \mathrm{nm}^{-1}$ according to SRIM while CasP predicts $50 \mathrm{keV} \mathrm{nm}^{-1}$.

The energy loss of $1 \mathrm{GeV}^{238} \mathrm{U}^{28+}$ ions in ta-C is only $22 \mathrm{keV} \mathrm{nm}^{-1}$ being barely above the track formation threshold. For this reason, thin degrader foils $(5 \mu \mathrm{m})$ were used, even if the beam energy was in the range of the Bragg peak, in order to increase the charge state to equilibrium values.

All samples, covered with aluminum degrader foils, were mounted onto $\mathrm{Al}$ sample holders ${ }^{4}$. The irradiation took place at room temperature. The beam energy deposited was about $3 \mathrm{~J} \mathrm{~cm}^{-2}$ for a fluence of $1 \times 10^{10} \mathrm{~cm}^{-2}$. If the resulting heat was absorbed by a silicon waver $(600 \mu \mathrm{m})$, the beam would have heated the sample by $30 \mathrm{~K}$. This raise in temperature had no effect on the samples and could be neglected. For safety reasons, the samples had to be stored until the radioactivity, caused by the activation of the substrates and sample holders, dropped to safe levels.

In addition to the radiations at the GSI, a few samples were irradiated at Ganil at Caen in France ${ }^{5}$. This facility provided a $\mathrm{Pb}$ beam $\left(4.57 \mathrm{MeV} \mathrm{u}^{-1}\right)$ with high charge states of $53+, 56+$ and $60+$. In order to achieve the high ionization states, the accelerated ions passed through a thin carbon foil (compare figure 2.3 $(a)$ prior to the charge separation by a bending magnet. The single charge state beams were aimed at the samples directly without any degrader in order to allow the analysis of the effect of the charge state on track formation. The fluences were in the order of some $1 \times 10^{9} \mathrm{~cm}^{-2}$.

\subsection{Transmission electron microscopy - TEM}

\subsubsection{Sample preparation}

Transmission electron microscopy (TEM) is used to characterize the structural composition of the films deposited by MSIBD. This microscopy method allows the direct analysis of properties such as lattice structure and composition of nanometer-sized volumes. A detailed description of theoretical and practical aspects of TEM is given by Williams and Carter 159 .

All samples analyzed in this work were prepared by Focused ion beam (FIB). For each sample, a wedge, vertical to the surface of the film, was cut out by sputtering with a focused Ga beam using the FIB Fei Nova Nanolab 600. In a first step, each sample was covered with Palladium (e-beam deposited) to protect the surface from sputtering. Two trenches were sputtered leaving a thin wedge. Finally, the

\footnotetext{
${ }^{4} \mathrm{Al}$ is used in swift heavy ion irradiation experiments because it is not as easily radioactively activated by nuclear reactions.

5 http://www.ganil-spiral2.eu/?set_language=en
} 
sample was attached to an Omniprobe, a microscopic movable tip, by gluing again with palladium. The specimen was transferred to a TEM sample holder (grid) and attached again by Pd-gluing. In a last step, the cross section of the wedge is thinned to be transparent to the TEM beam. The preparation is described in more detail by Zutz 157 .

\subsubsection{TEM-imaging}

The samples were analyzed in a $200 \mathrm{keV}$ Philips CM-200-FEG-U/T capable of resolving to the atomic level $(0.11 \mathrm{~nm})$. The micrographs were recorded by a fiber-optic coupled and peltier cooled CCD-camera from Gatan. The wedges prepared by FIB offered a cross section view of the films, allowing for structural analysis (e.g. cluster formation) and the measurements of film thicknesses. To achieve the later to sufficient precision, the samples were oriented using the crystalline structure of the Si-substrates orientated in [111]-direction.

\subsection{X-ray photoemission spectroscopy - XPS}

$X$-ray photoemission spectroscopy (XPS) is a standard characterization method for chemical composition and bond structure. The sample is irradiated with X-ray photons which excite the electrons of the specimen. If the photon electron interaction is close to the surface, the electron can escape into the vacuum according to the photoelectric effect described by Einstein [160]. The kinetic energy of the electrons is measured and the binding energy can be determined.

During the photon electron interaction, the photon is captured and its energy $h \nu$ is transferred to the electron which in turn can leave its orbit, if the energy is larger than the binding energy $E_{\text {bind }}$. The remaining kinetic energy of the electron is

$$
E_{\text {kin }}=h \nu-E_{\text {bind }}-\Phi,
$$

where $\Phi$ is the work function. The binding energy is relative to the Fermi energy and the work function is the barrier height between $E_{\mathrm{F}}$ and the vacuum. In other words, $\Phi$ is the energy required for an electron at the Fermi energy to escape into vacuum. The XPS detector scans the kinetic electron energy and maps the rates. The result is an energy dependent electron count which can be used to determine the chemical composition and stoichiometry of the sample. For each binding energy a peak is measured in the XPS spectra. An example of a spectrum is shown in figure 3.2 showing two peaks, which are the result of the $4 \mathrm{f} 5 / 2$ and $4 \mathrm{f7} / 2$ levels of $\mathrm{Au}$. The energy scale is transformed to show $E_{\text {bind }}$, therefore, the kinetic energy of the electrons increases towards the right while the measured kinetic energy decreases. The intensity scale is normalized and presented in arbitrary units.

In addition to the binding energy, the plasmon energy (frequency) can be extracted of an XPS measurement. If an escaping electron excites a plasmon, it will lose the 


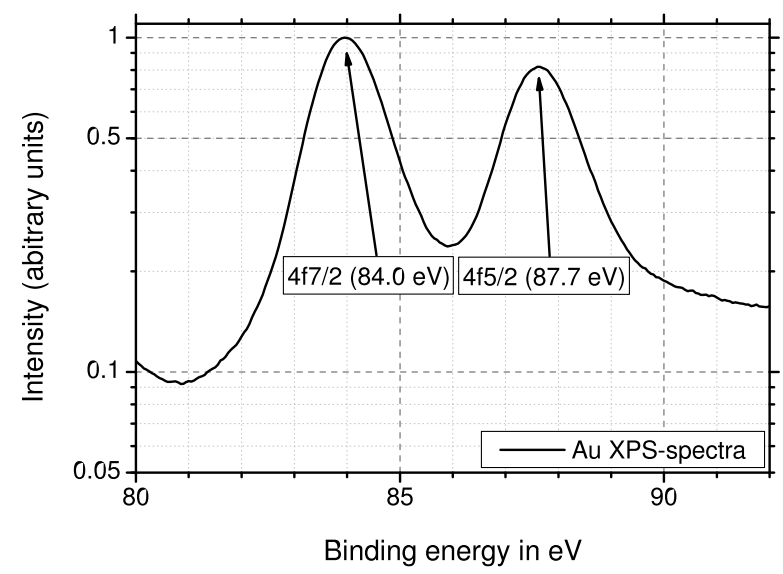

Figure 3.2: XPS spectra of sputter-cleaned Au target used for calibrating the energy. The spectrum shows the $4 \mathrm{f5} / 2$ and $4 \mathrm{f} 7 / 2$ peaks at $84.0 \mathrm{eV}$ and $87.7 \mathrm{eV}$. The data was corrected by a constant to fit these values.

plasmon energy $\left(\hbar \omega_{\text {plas }}\right)$. Theoretically, multiple plasmon excitations are possible, however, the likelihood of detected electrons decreases with increasing plasmon number and the signal is often undetectable for higher orders. For the first plasmon excitation, the kinetic energy of the electron is:

$$
E_{\text {kin }}=h \nu-E_{\text {bind }}-\Phi-\hbar \omega_{\text {plas }}
$$

The plasmon energy depends on the electron density $n$ and the permittivity $\epsilon$ :

$$
\omega_{\text {plas }}=\sqrt{\frac{n e^{2}}{m_{\mathrm{e}} \epsilon}},
$$

where $e$ is the elementary charge and $m_{\mathrm{e}}$ the (effective) electron mass.

Although the X-ray photons may penetrate the sample deeper, the mean free path of the escaping electrons is limiting the analysis depth. For electrons in the order of $1000 \mathrm{eV}$, the mean free path is about $1 \mathrm{~nm}$ to $2 \mathrm{~nm}$ [161]. Therefore, the effective signal depth of XPS spectra can be estimated to be less than $5 \mathrm{~nm}$.

Electrons involved in inelastic collisions before escaping the sample will be detected as random background in the spectra. Typically, towards lower energies of a peak, the background raises because of inelastic collisions, as seen in figure 3.

The precision of the spectra depends on three factors: Statistical count fluctuation, energy resolution of the detector, and absolute energy calibration. The statistical

\footnotetext{
${ }^{6}$ The higher background is towards higher binding energy which corresponds to a reduced kinetic energy of the electron.
} 
fluctuation introduces a high frequency noise to the intensity of the $E_{\text {kin-spectra, }}$ which can be reduced by longer data acquisition. The limited energy resolution of the detector will convolute sharp peaks usually with Gaussian distributions. The energy calibration is achieved by measuring a known binding energy $(\mathrm{Au})$ and calibrating the detector. A false calibration will lead to systematic energy shifts of the spectra. This shift limits the precision of absolute peak position, while it has no effect for relative energy differences such as the plasmon energy.

The data in figure 3.2 shows a spectrum of a calibration measurement. A gold target was sputter cleaned and measured. The peak positions of the $4 \mathrm{f} 5 / 2$ and $4 \mathrm{f} 7 / 2$ electrons were calibrated to literature values of $84.0 \mathrm{eV}$ and $87.7 \mathrm{eV} 1627$. By looking into various databases, e.g. the National Institute for Standards and Technology [163], a fluctuation of the values of a few tenth electronvolt is revealed limiting the precision of the calibration.

ADONIS is equipped with an in-situ XPS chamber $\left(5 \times 10^{-9}\right.$ mbar $)$ to analyze newly deposited films. The Omicron DAR400, an X-ray source with a $\mathrm{Mg}-\mathrm{K}_{\alpha}$ line, was used as well as an Omnicron EA125 hemispherical detector. Further descriptions of this particular setup were written, for example, by Ronning et al. [164].

\subsection{Electrical characterization}

Electrical characterizations performed for this work were conducted with two setups, one is capable to measure in the temperature range of $15 \mathrm{~K}$ to $300 \mathrm{~K}$ (low temperature setup), while the other (high temperature setup) covers the temperature range of $295 \mathrm{~K}$ to $390 \mathrm{~K}$.

\section{Low-temperature setup}

The low-temperature setup was constructed by Nix [32]. It has been used and described in further works by Nix [25, 33], Gehrke [27], and Brötzmann [103]. The temperature is controlled by a closed cycle He-cryostat and a combined temperature controller and heater (Lakeshore 331 Temperature Controller). The cold-heat of the cryostat is located in a vacuum chamber $\left(<1 \times 10^{-6} \mathrm{mbar}\right)$, on which the samples are mounted using a sapphire carrier. The temperature is measured with two semiconductor diodes. The chamber offers six feedthroughs for electrical probing. A Keithley 237 Source Measurement Unit (SMU) is used to perform the electrical measurements. The cold-heat with a sample carrier and the glued contacts is shown in figure $3.3(b)$.

The temperature controller, the SMU, and an additional relay switch are connected to a computer to control and run the experiment. Self-written software, capable of

\footnotetext{
${ }^{7}$ also online available: https://userweb.jlab.org/ gwyn/ebindene.html.
} 
performing measurements at multiple temperatures in a batch-mode, can be configured to run temperature dependent measurements autonomously. The relay switch allows the probing of different contact pads (pads) in a single run.

To conduct a typical experiment, the Software is started and the desired temperatures can be entered in a list. Usually, each temperature is measured twice once during the cooling cycle and again while heating back to room temperature. The consistency of the cooling and heating cycle data indicates stable contacts and clean temperature readings. Usually, more than one contact of the sample should be analyzed; each pad is connected to a different relay of the switch box. At each temperature multiple characteristics are recorded to ASCII-files for all pads depending on user configuration of the software. Each pad can be examined using independent SMU parameters. The software performs either $I-V$ - or $V$ - $I$-characteristics. In either case each measurement consists of three data ranges: zero to max, max to min, and min to zero. All three data arrays make up one sweep resembling an $I-V$ - or a $V$-I-characteristic. Sweeping up and down helps detecting charging effects of the sample during measurements, seen as hysteresis. In this work all sweeps recorded are $I$ - $V$-characteristics.

\section{High temperature setup}

The high temperature setup is relatively crude, but still efficient. The experiments in this temperature regime were performed at ambient atmosphere in a small cylindrical aluminum container connected to a hot plate. This configuration is shown in figure 3.3(c). A lid, not seen in the picture, can be closed to seal the "chamber". On the sides of the cylinder five groves are cut into the aluminum for the pressure contacts and temperature sensors. The temperature is measured using two type-k thermocouples and a Voltcarft K204 controller, which features a serial port. The heater is powered by a variable current source $(3 \mathrm{~A}$ and $30 \mathrm{~V})$ and the $I-V$-probing of the sample is done either with the Keithley 237 or the Keithley 2611. The heated cylinder is housed in a larger aluminum box to provide more stable environmental conditions (no convection).

This experiment is controlled by a self-written python script recording $I$ - $V$-characteristics at specific temperatures. Unlike the low temperature setup, the heating power cannot be remotely controlled and has to be controlled manually. To perform measurements above room temperatures, the script header has to be edited first. The desired temperatures must be entered as well as the SMU parameters ( $\mathrm{min} / \mathrm{max}$ voltages, voltages steps etc.). The script is started and waits for the first temperature to be reached. The heater is set to a small power to ensure a slow temperature gradient of about $1 \mathrm{~K} \mathrm{~min}^{-1}$. As soon as the temperature is reached, the SMU is triggered and performs the sweep saving the data to ASCII files. It is of importance to choose SMU parameters to perform the sweep as fast as possible (i.e. $5 \mathrm{~s}$ ). During this time span, the temperature is almost constant, if the ramp is not too steep. As the 
temperature rises, the heating power has to be increased gradually until the maximum is reached. The script automatically cuts the power using the relay switch after measuring the highest temperature. While cooling, a second set of characteristics is recorded. In case of correct configuration and a good measurement, both heating and cooling runs deliver the same results. In this case it can be assumed that the sample did not suffer from heating damage, the contacts are still intact and the temperature measurement did not suffer from inertia effects of badly placed sensors 8 . Another instrument of ensuring correct temperature measurement is the second temperature sensor, which measures the air temperature in the small sample chamber. During an experiment, the sample temperature should not excite the chamber temperature by more than $5 \mathrm{~K}$ to avoid incorrect measurements.

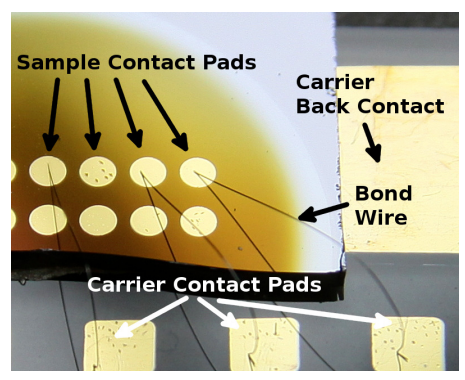

(a) Carrier in low temperature setup.

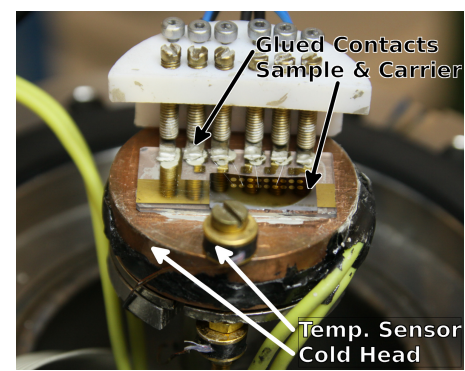

(b) Sample on sapphire carrier.

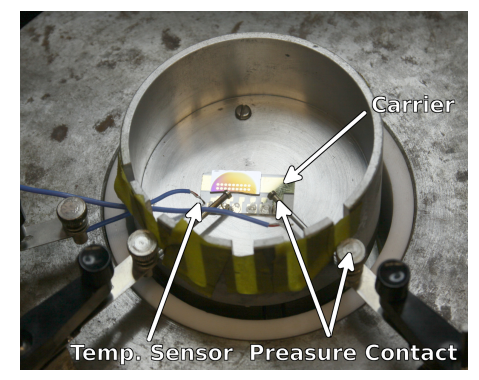

(c) Carrier in high temperature setup.

Figure 3.3: Photographs of the sample mounted on the sapphire carrier (a). The pads for the top contacts are connected with bond wires to the carrier. The sample is fixed to the carrier with conductive silver. This sample arrangement can be mounted in the low temperature setup (b). Here, the holder is glued to the cold heat using conductive silver. The pads of the carrier are also connected with conductive silver. Next to the sample, the temperature is measured using the semiconductor diode. In (c) the high temperature setup is shown. The blue wires are the type-K thermo-sensors, two pressure contacts are shown to connect the carrier to the SMU. The extra groves of the cylinder are closed with tape to reduce convection inside the container.

\section{Contact pads}

To contact the top side of the films, contact pads were evaporated on top of the samples. First, about $10 \mathrm{~nm}$ of chromium was deposited onto the sample by e-beam evaporation. The chromium ensures good adhesion to the ta-C. In a second step, $100 \mathrm{~nm}$ gold was deposited on top of the chromium to seal the pad and avoid degradation of the contacts due to oxidation. The process was done in vacuum in the

${ }^{8}$ If the sensor does not measure the sample temperature, but for example, the temperature of the air directly above the sample, the true sample temperature will not be the same for the sensor reading during cooling and heating. 
range of $1 \times 10^{-6}$ mbar to $1 \times 10^{-5}$ mbar. The sample holder for the evaporation chamber can be equipped with different masks. For most measurements in this work round contact pads with a diameter of $0.6 \mathrm{~mm}$ were used. The contact masks were fabricated by the workshop of the II. Physical Institute from Copper foil using small drills and a precision mill.

\subsubsection{Carriers}

The carriers for the samples during electrical characterization were made of sapphire. On top of the holder different chromium-gold pads were evaporated (same method as used on samples) as seen in figure $3.3(a)$. The large pad on top the carrier was used to fix the sample, the smaller were used for connecting the carrier to the SMU.

\subsubsection{Sample back-contacts}

The samples were glued onto the holder using conductive silver giving a good electrical and thermal contact. The high doping level of the Si-substrates reduces the effect of the Schottky contact to the silver and gold to such a degree, that the reverse-bias resistance is in the order of $10 \Omega$. In case of highly conductive samples (tracks), a four-point setup was used (chapter 3.5.3).

\subsubsection{Wedge bonding}

The wedge bonder, a device capable of creating contacts to micrometer sized pads, was used to connect the evaporated pads of the sample to pads on the sample holder. The bond wires used had a diameter of $30 \mu \mathrm{m}$. A typical bond connecting the ta-C sample showed a resistance of about $1 \Omega$ and was stable up to $300 \mathrm{~mA}$. If a higher current was required during measurements, two bonds were placed in parallel. A third bond was connected to a different pad on the carrier as a sense for a four-point measurement (see chapter 3.5.3).

\section{Local and Remote Setup}

In general, samples are connected with two leads to perform electrical characterization. In this two-point setup, the resulting resistance is the sum of resistances of the sample, contacts, and wires used to connect to the equipment. This setup works well if the contact resistance is small compared to the sample resistance. Otherwise, a four-point setup is required. In this case, for each contact (top and back) two leads are required. One will transport the current (output) while the other is used to probe the voltage (sense). A high-impedance voltmeter was used in the (G $\Omega$-range). The four-point setup is also called remote probing because of the remote voltage probing, whereas the two-point geometry is referred to local probing. 
In case of the ta- $\mathrm{C}$ samples the remote setup was realized by adding a contact onto the free silicon at the edge of the sample where no film was deposited (sense back-contact). Because of the high conductivity of the Si used, the potential within the substrate can be considered constant, the exact position of the sense-contact is irrelevant. Also creating two separate back-contacts, did not show different results. An additional bond was added to the top contact pads. Nonetheless, the resistance of the interface from silicon to ta- $\mathrm{C}$ and from ta- $\mathrm{C}$ to chromium remains in the circuit in this remote setup.

In case of some measurements, the extra sense bond on the top contact was omitte$\mathrm{d}$ At the same time, the separate back contacts were kept to compensate the resistance of the conductive silver. This method, referred to as three-point setup, was used for measuring samples with resistances exceeding $500 \Omega$. The advantage of this setup is the reduced demand for wires and feedthroughs of the vacuum chamber allowing more parallel measurements per run. Assuming a top-contact-resistance of $2 \Omega$ leads to errors less than $0.5 \%$.

\section{Source measurement units}

Two different Source Measurement Units SMU were available. The default unit, Keithley 237, offers a voltage range of $-1100 \mathrm{~V}$ to $1100 \mathrm{~V}$ and a maximal current of $100 \mathrm{~mA}$. The Keithley 2611 has a maximal voltage of $110 \mathrm{~V}$ offering a current of $1 \mathrm{~A}$. In case of the high temperature investigations of the irradiated samples, the highcurrent SMU was used. The current was limited by the bond wires to $600 \mathrm{~mA}$. Both SMU offering local and remote probing are sensitive enough to measure the relevant voltages and currents. The setup-equipment (feedthroughs, wiring, and shielding) is not designed for the pA-range. Measurements of currents lower than $0.01 \mathrm{nA}$ become erroneous, although the Keithley 237 should be able to measure smaller currents.

\section{Characteristics of substrate}

All samples characterized were grown on n-Si with a resistivity of $5 \mathrm{~m} \Omega \mathrm{cm}$ equivalent to $20 \mathrm{kS} \mathrm{m}^{-1}$. The low resistivity and the high degeneration of the semiconductor should provide an almost metallic contact. In figure 3.4 the resistance of a contact pad (1.1 mm diameter) directly evaporated onto the silicon is shown. The substrate was cleaned with acetone to remove organic debris. Shortly before mounting in the evaporation chamber, the silicon was etched with $1 \%$ HF to remove any native oxide. The sample was contacted in remote-setup mode onto the sapphire sample holder. All measured $I$ - $V$-characteristics exhibit ohmic-behavior.

As expected for semiconductors, the resistance of the substrate increases towards low temperatures. Overall, the resistance of the contacts of the four-point setup and the substrate are very small and can be neglected, except for very small resistances of the order of $1 \Omega$. These values are only reached by irradiated samples at the highest 


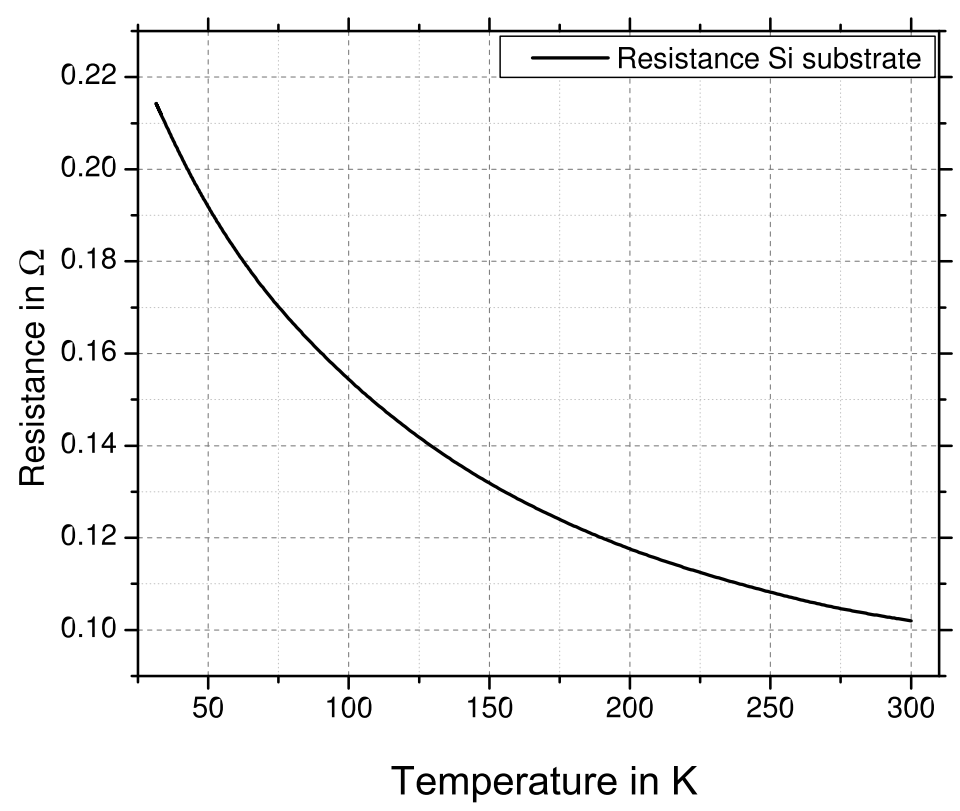

Figure 3.4: Resistance of Silicon substrates used for all samples. A Cr/Au contact pad was directly evaporated onto a the cleaned Si surface. The data was acquired in four-point configuration.

temperatures. Nonetheless, this effect has been neglected, because the substrate resistance makes up less than $10 \%$ of the measured values.

\subsection{Atomic force microscopy - AFM}

The Atomic Force Microscopy (AFM) [165] is a high-resolution scanning probe microscopy method, where a small nanometer-sized tip is moved over the sample surface. The tip height is measured and used to map the surface topography with sub-nanometer precision.

The analysis of this work was performed using the XE-100 by PSIA at the Hochschule Harz - Hochschule für angewandte Wissenschaften $(F H)$ in Wernigerode. The microscope is housed in a sound insulated containment and is carefully decoupled from the building to reduce mechanical vibrations. Depending on tip diameter, the system resolves about $5 \mathrm{~nm}$ laterally and $0.1 \mathrm{~nm}$ vertically. This precision is sufficient to record the hillocks of the ion tracks in ta-C .

In addition to topographical information, the AFM in Wernigerode is equipped with an electric characterization setup using microscopic conductive tips. This feature allows the individual analysis of conductive ion tracks in ta-C . The setup is 
capable of measuring an $I$ - $V$-characteristic of one position (e.g. an ion track). Alternatively, a fixed bias can be applied while scanning, the locally resolved current is recorded creating a current map of a surface. Here, the identification of conductive ion tracks as well as the comparison of their resistances is possible. A recent addition to the AFM setup in Wernigerode was a temperature controlled stage allowing sample heating up to $100^{\circ} \mathrm{C}$.

The technical difficulty is the detection of low currents in the pA regime and below while keeping a fast sampling rate in the order of $200 \mathrm{~Hz}$. This rate is necessary to keep up with typical scan rates of 0.6 lines per second and 256 samples per line. Even while measuring local $I-V$-characteristics, a relatively fast analysis is required because a minimal sample drift would otherwise reduce the spacial resolution. The current is measured using an operational amplifier close to the tip allowing a current resolution of $0.3 \mathrm{pA}$. The maximal current is $100 \mathrm{nA}$. This range is adequate for the characterization of typical ion tracks in ta-C.

All ta-C films were analyzed in contact-mode using Pt/Ir-film coated tips (BSEM75 and MULTI75E) by Budget Sensors ContE tips allowing parallel electric and topographic characterization. The temperature in the AFM chamber is about $305 \mathrm{~K}$ in case of temperature dependent measurements, this temperature was raised up to 373 K. All AFM data processing was done using Gwyddion $9^{9}$ an Open Source (GNU) application for scanning probe microscopy.

${ }^{9}$ http://gwyddion.net/ 



\section{Structural characterization by XPS}

The XPS was performed on the sample for two reasons. First, to check the copper content in the films and check the $\mathrm{sp}^{3}$ ratio of the ta-C in dependence of the copper concentration. The $\mathrm{sp}^{3}$-bond to $\mathrm{sp}^{2}$-bond ratio can be determined by measuring the C-1s peak, which is different for Graphite $(284.4 \mathrm{eV})$ and diamond $(285.2 \mathrm{eV})$ [166]. Typically, in XPS measurements of amorphous carbon films, a wide peak around the two energies is detected. The bond ratio can be estimated by fitting two distributions to the data centered at the $\mathrm{sp}^{2}$ and $\mathrm{sp}^{3}$ energy. This method was applied, for example, by Ronning et al. [51, 164] and Gerhards et al. [153 using samples synthesized with ADONIS. Unfortunately, careful calibration of the energy scale and good statistics are required. The XPS detectors available were at the end of their lifetime while the measurements for this work were performed. The aged detectors showed a higher noise level and lower electron count. For this reason, only the peak position was measured depending on copper concentration offering a first order approximation of the change in hybridization ratio.

The plasmon frequency is also different for $\mathrm{sp}^{2}$ and $\mathrm{sp}^{3}$ hybridized electrons. Amorphous carbon exhibits a plasmon frequency between the values of graphite $(26.0 \mathrm{eV})$ and diamond $(33.8 \mathrm{eV})$, which is according to Berger et al. 167] and Reinke et al. 168 in the order of $30 \mathrm{eV}$. Figure 4.1 shows the XPS spectra of the C-1s peak and the first plasmon oscillation for the ta-C films with different copper concentrations. All spectra show the $\mathrm{C}-1 \mathrm{~s}$ peak close to $285 \mathrm{eV}$. The inlet magnifies the peak and reveals a small fluctuation, which is better visible in figure 4.2 $(a)$. The plasmon-peak is at about $316 \mathrm{eV}$, shown in more detail in figure 4.2(b). All spectra look overall very similar, the only exception is the sharper plasmon peak of the $5 \%$ copper film.

In figure 4.2(a), the peak positions of the C-1s peaks are shown with margins of error. The main source of error is the energy calibration, which causes a systematic displacement. The spectra of $0 \%$ and $0.1 \% \mathrm{Cu}$ were calibrated individually. The higher copper concentrations were measured in a row and were calibrated to a single $\mathrm{Au}$ spectrum. As a result, the trend seen for high copper concentrations has a small error, only the absolute energy position of all peaks have an uncertainty of $0.1 \mathrm{eV}$. Keeping this in mind, the data shows a constant C-1s peak position up to $2 \%$ within the margin of errors. At $5 \% \mathrm{Cu}$, the peak energy drops by about $0.1 \mathrm{eV}$. The C-1s energy of $285 \mathrm{eV}$ to $285.1 \mathrm{eV}$ corresponds to high $\mathrm{sp}^{3}$ hybridization ratio of about $80 \%$ [51, 168. This result is supported by the energy dependence of the plasmon frequency shown in figure 4.2(b), which depends as well on the hybridization ratio 167, 168]. Again, up to $2 \% \mathrm{Cu}$ concentration the plasmon frequency is constant within the margin of error. At $5 \% \mathrm{Cu}$, the energy drops slightly. Assuming a linear 


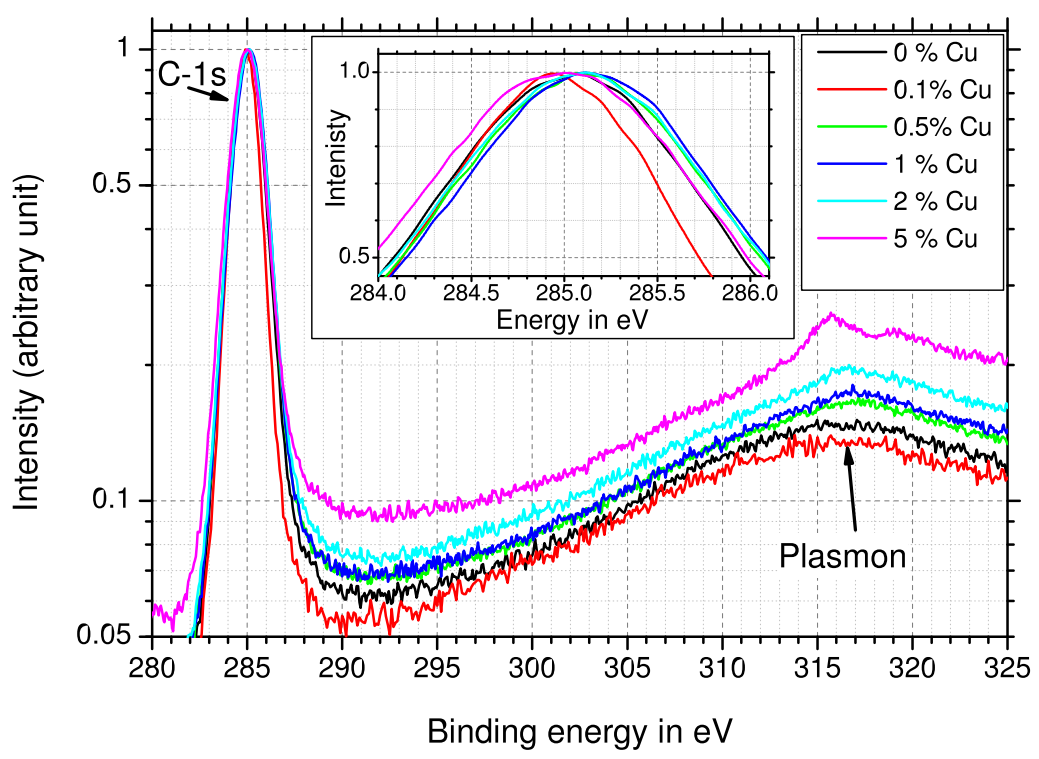

Figure 4.1: XPS spectra of the C-1s peak and the first plasmon oscillation of the ta-C films with different $\mathrm{Cu}$ content. The position of both peaks is almost independent of $\mathrm{Cu}$ concentration, only a slight shift of the plasmon energy at $2 \%$ and $5 \%$ is visible. The fluctuation of the C-1s peak in the insert is mainly caused by the systematic errors of the energy-scale calibration. The peak with is constant, which suggests a constant $\mathrm{sp}^{2}-\mathrm{sp}^{3}$ ratio for all $\mathrm{Cu}$ concentrations. The background increases with copper concentration.

dependence of the plasmon energy on the $\mathrm{sp}^{3}$ content [50] suggests a $\mathrm{sp}^{3}$ content of about $75 \%(31.8 \mathrm{eV})$ for samples with low copper concentrations and $60 \%$ for the sample with $5 \% \mathrm{Cu}$. The expected $\mathrm{sp}^{3}$ hybridization of $100 \mathrm{eV}$ ta-C is assumed to be $80 \%$ to $90 \%$ [37]. The lower values in the present XPS measurements are caused by the extreme surface sensitivity and the increased $\mathrm{sp}^{2}$ hybridization at the surface of the ta-C films.

In figure 4.3 XPS spectra displaying the $\mathrm{Cu}-2 \mathrm{p} 1 / 2$ and $\mathrm{Cu}-2 \mathrm{p} 3 / 2$ peak at $933.1 \mathrm{eV}$ and $953.0 \mathrm{eV}$ are shown for all copper concentrations. The copper-free sample shows no peaks and for all other samples, the peak height increases with copper concentration. The peak area can be used to estimate the copper content at the surface of the films comparing the peak area of the $\mathrm{C}$ - 1 s peak and the $\mathrm{Cu}-2 \mathrm{p} 3 / 2$ peak area for each measurement, using an estimation for the different cross-sections [169]. All spectra were normalized in order to show a direct comparison of the copper peaks. The increasing background is the result of inelastic events creating tails at lower energies.

The quantitative results are shown in the insert of figure 4.3 compared to deposited amount of copper. The values are larger than the expected stoichiometries calculated from the deposited amounts during preparation. However, the XPS provides only information of the very near surface. Copper is known to accumulate at the surface of ta-C during MSIBD. Gerhards et al. 153 calculated similar results from XPS 


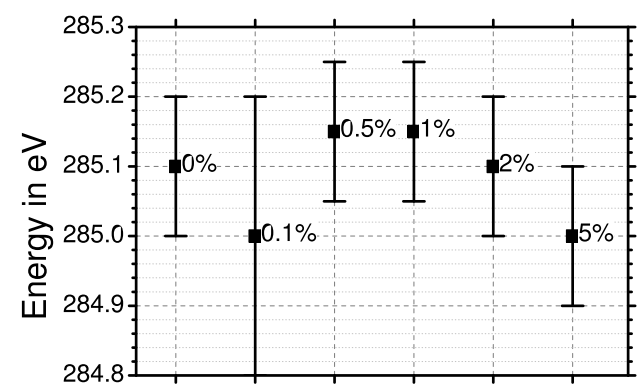

(a) $\mathrm{C}$-1s peak position depending on $\mathrm{Cu}$ concentration.

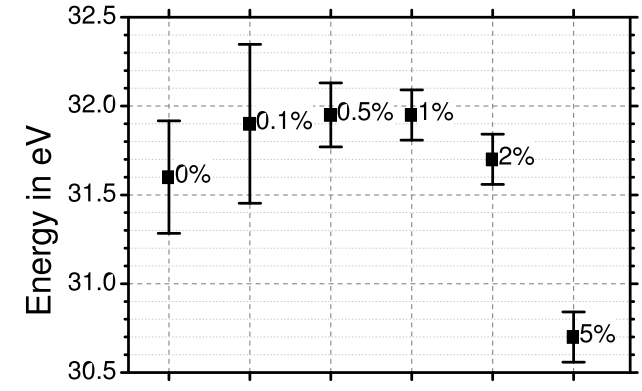

(b) Plasmon energy depending on $\mathrm{Cu}$ concentration

Figure 4.2: XPS spectra showing the $\mathrm{C}-1$ s peak energy depending on $\mathrm{Cu}$ concentration (a), a shift towards lower energies would suggest an increasing sp ${ }^{2}$ content. Only at $5 \% \mathrm{Cu}$, the value seems to drop slightly. In addition, the plasmon energy (b) is also dependent on hybridization ratio. Again, a lower value correlates to a higher $\mathrm{sp}^{2}$ content. The plasmon energy is constant up to $2 \%$ and drops at $5 \% \mathrm{Cu}$.

spectra. In addition, they checked the stoichiometry using Rutherford-Backscattering (RBS). This method is sensitive to the total film volume and not just the surface. Hereby, they found a copper concentration close to the actually deposited amount. Due to sputter loss, the values are slightly smaller $(10 \%)$ than deposited agreeing with the theoretical model of Gerhards et al. [158].

The spectra show two additional peaks (a) and (b) in figure 4.3 which are caused by an additional line of the X-ray source emitting a second energy. Therefore, (a) and (b) are the blind peaks of $\mathrm{Cu}-2 \mathrm{p} 3 / 2$ and $\mathrm{Cu}-2 \mathrm{p} 1 / 2$ respectively. Especially, the peak at (b) has a high shoulder increasing the background in turn decreasing the precision of stoichiometry comparisons of the copper content.

In conclusion, the XPS-data of the $\mathrm{C}-1$ s peak and the plasmon oscillation show a constant $\mathrm{sp}^{3}$ content up to $2 \% \mathrm{Cu}$ of about $80 \%$. At $5 \% \mathrm{Cu}$, the $\mathrm{sp}^{3}$ ratio starts to drop by about $10 \%$. This result is in good agreement with investigations by Gerhards et al. [153] and Ronning [51]. The copper concentration detected by XPS measurements is higher than expected from the deposition rate. The deviation is caused by the tendency of copper to accumulate close to the surface. Gerhards et al. showed by RBS measurements a $\mathrm{Cu}$ concentration slightly less (10\%) than the deposited amount in the range of $0 \%$ to $5 \% \mathrm{Cu}$. Because of her experiments, it is assumed that the deposited amount of copper matches the incorporated amount in first order approximation.

An exception of this assumption occurs for the samples with $0.1 \% \mathrm{Cu}$. These samples were contaminated inadvertently by sputter depositing $\mathrm{Cu}$ during the initial substrate-cleaning process. During the subsequential ta- $\mathrm{C}$ deposition, no $\mathrm{Cu}$ was co- 
deposited as done with the other samples. However, the tendency of $\mathrm{Cu}$ to accumulate at the surface of ta-C during deposition diluted the initial contamination through the entire film. The $0.1 \%$ value was solely derived by XPS measurements and is a first order approximation only. Most likely, the actual average copper concentration is less.

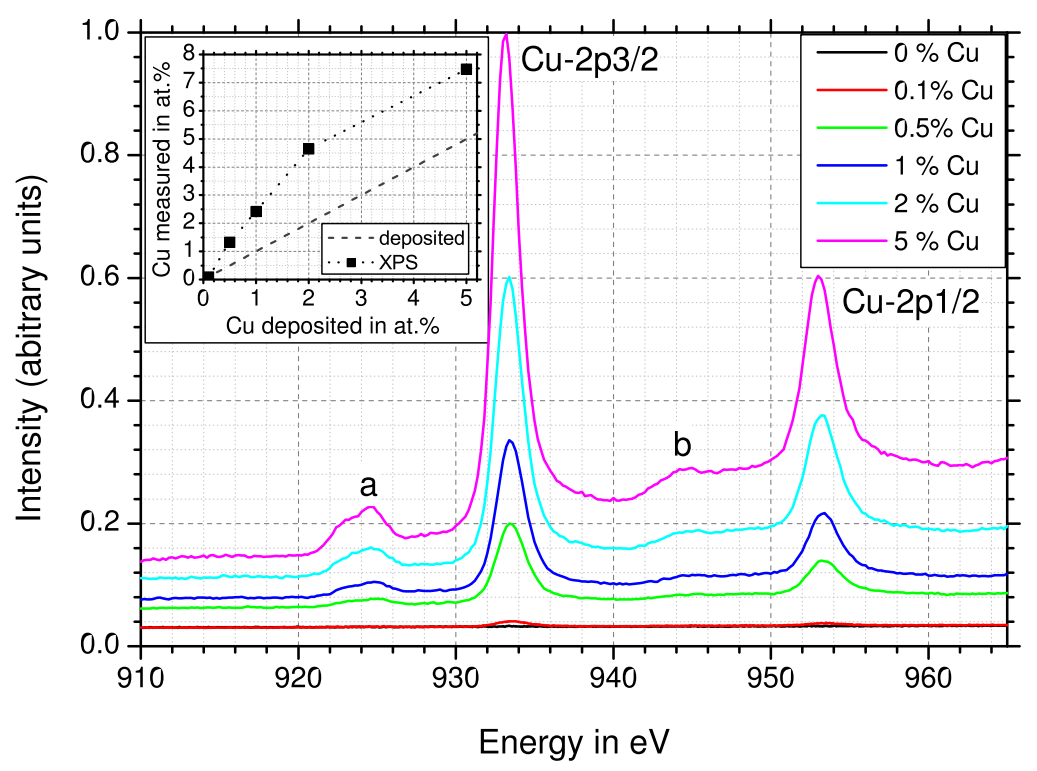

Figure 4.3: XPS spectra of the $\mathrm{Cu}-2 \mathrm{p} 3 / 2$ and $\mathrm{Cu}-2 \mathrm{p} 1 / 2$ peaks of the copper impurities in ta-C. The spectra were normalized using the $\mathrm{C} 1$-s peak of the ta- $\mathrm{C}$ to compare the total $\mathrm{Cu}$ concentration. The insert shows the calculated $\mathrm{Cu}$ concentration near the surface, which is higher than expected by deposition ( $\mathrm{Cu}$ accumulates at the surface). The small peaks marked (a) and (b) are blind peaks of the two $\mathrm{Cu}-2 \mathrm{p}$ peaks, caused by second photon energy of the X-ray source. 


\section{Macroscopic electrical characterization of ta-C conductivity}

The electrical characterization of ta-C, grown by MSIBD, is well established. The first measurements performed with the equipment used in this work were performed by Nix [32, 33]. The sample preparation was improved during the diploma thesis of Gehrke [27] and Nix $\mathrm{PhD}$ thesis [25]. In particular, the adhesion-enhancing chromium film was added to the contact pad, the leads were created by wedge bonding instead of gold wires glued with conductive silver. Finally, the remote probing of the bias-voltage was implemented. All these measures led to smaller impacts of contact resistances and more stable, reproducible results in general. Furthermore, the temperature-dependent setup for high temperatures was much improved to yield stable and accurate temperatures to a precision of $0.3 \mathrm{~K}$. Earlier measurements above room temperature performed with the available equipment could not control the temperature well because of a crude heating controller and temperature probing system.

\subsection{Unirradiated virgin ta-C}

At first, the electrical properties of the pure ta-C are of interest. Figure 5.1 shows the temperature dependence of the current measured at different potentials. The film thickness was about $85 \mathrm{~nm}$ and the contact pad had a diameter of $0.9 \mathrm{~nm}$ $\left(6.37 \times 10^{-3} \mathrm{~cm}^{2}\right)$. The current decreases by five to seven orders of magnitude (depending on the applied field) in the measured temperature regime. Especially at higher voltages, the lines exhibit a kink at $300 \mathrm{~K}$. At this temperature, the two measurements of the low- and high-temperature setup were merged. The measurement was performed in the three-point setup (see chapter 3.5.3) and the contact resistances of both setups were slightly different. The temperature offset was less than $0.3 \mathrm{~K}$. Both measurement cycles were consistent for the cooling and heating run. The small offset is caused by the delicate contacting procedure. The results are in accordance with data of unirradiated ta-C shown by Gehrke 27] and Brötzmann [103. However, compared to old measurements of Nix [32, 33] and Schwen [22], the measured current has increased about a factor $100^{1}$. The temperature dependence is comparable, at $1 \mathrm{~V}$ the current drops by about four orders of magnitude between $300 \mathrm{~K}$ and $100 \mathrm{~K}$ in both the old and current measurements. Therefore, either the additional contact resistance had the same temperature dependence as the ta- $\mathrm{C}$ or, more likely, the contact pads had a much smaller effective contact area due to the missing

\footnotetext{
${ }^{1}$ comparing the currents at $300 \mathrm{~K}$ at $1 \mathrm{~V}$
} 
chromium layer. At low temperatures at $20 \mathrm{~K}$ to $40 \mathrm{~K}$, the curves show a king hinting to a residual conductivity. An interesting property of this residual conductivity is the strong variation between different contacts pads on a single sample. Two main factors are substrates roughness and quality of the film. The residual conductivity increases if rough and contaminated substrates are used. In this work, contact pads with the lowest residual conductivity available were chosen for further examination and presentation. This effect is discussed later in more detail (chapter 5.10).

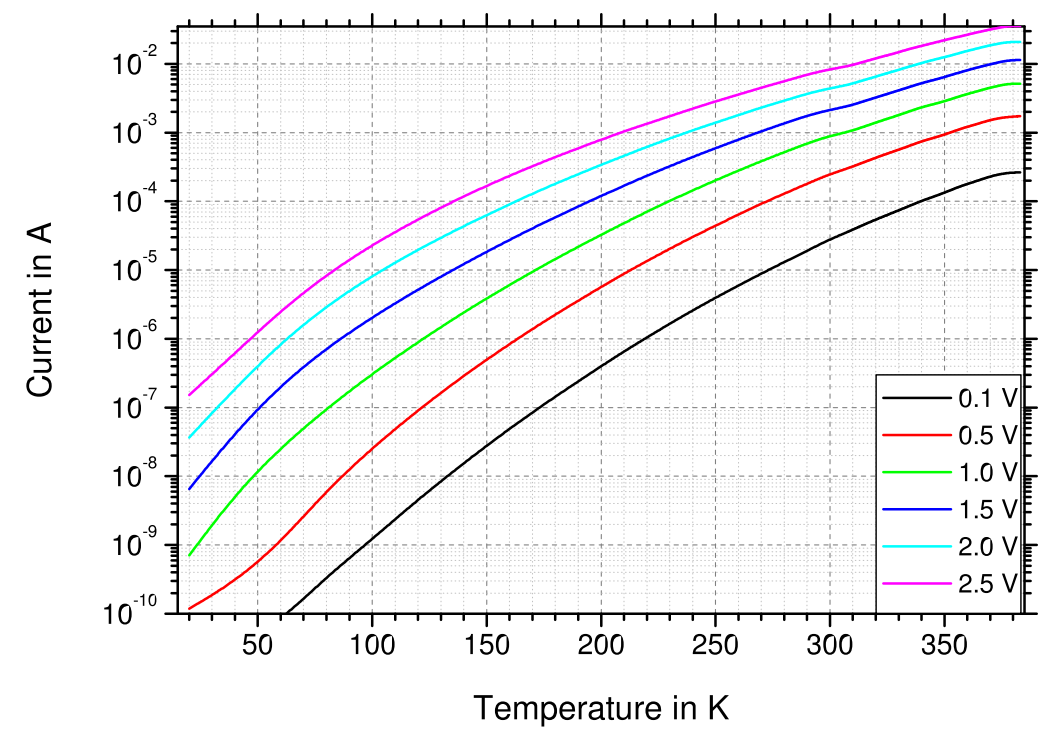

Figure 5.1: $I$ - $T$-plot of the data measured on a contact pad on a virgin ta-C film $\left(6.37 \times 10^{-3} \mathrm{~cm}^{2}\right.$ contact and $85 \mathrm{~nm}$ film thickness). The conductivity decreases by 5-7 orders of magnitude towards low temperatures. The kink at $300 \mathrm{~K}$ is caused by the merging of the low- and high-temperature measurements. The kink at very low temperatures is caused by the residual conductivity (chapter 5.10 .

Some $I$ - $V$-characteristics of the same measurement are shown in the following figure 5.2. All characteristics are s-shaped and almost symmetric, as expected for such a ta-C film from previous publications and experiments. Earlier, in figure 3.4 the low resistivity of a chromium gold contact on the n-silicon was shown. This experiment did not suggest the formation of a Schottky contact between the silicon substrate and the evaporated contact pad. The symmetric characteristics of the ta-C film suggest an ohmic behavior of the contact pads and the silicon-ta-C interface as well. As analyzed by Brötzmann in more detail [103], a well pronounced heterojunction structure is formed by metal, ta-C, and less doped silicon. In addition, the first measurements performed by Nix showed symmetric characteristics for ta-C films on highly doped n-Si suggesting symmetric contacts although a large contact resistance was present. 
In fact, unsymmetrical characteristics measured on ta-C samples prepared on highly doped n-Si suggested a bad sample or inadequate conductivity of the leads. Samples degrade if too large fields are applied, subsequently slightly asymmetric characteristics are observed. This effect is illustrated in chapter 5.10 .

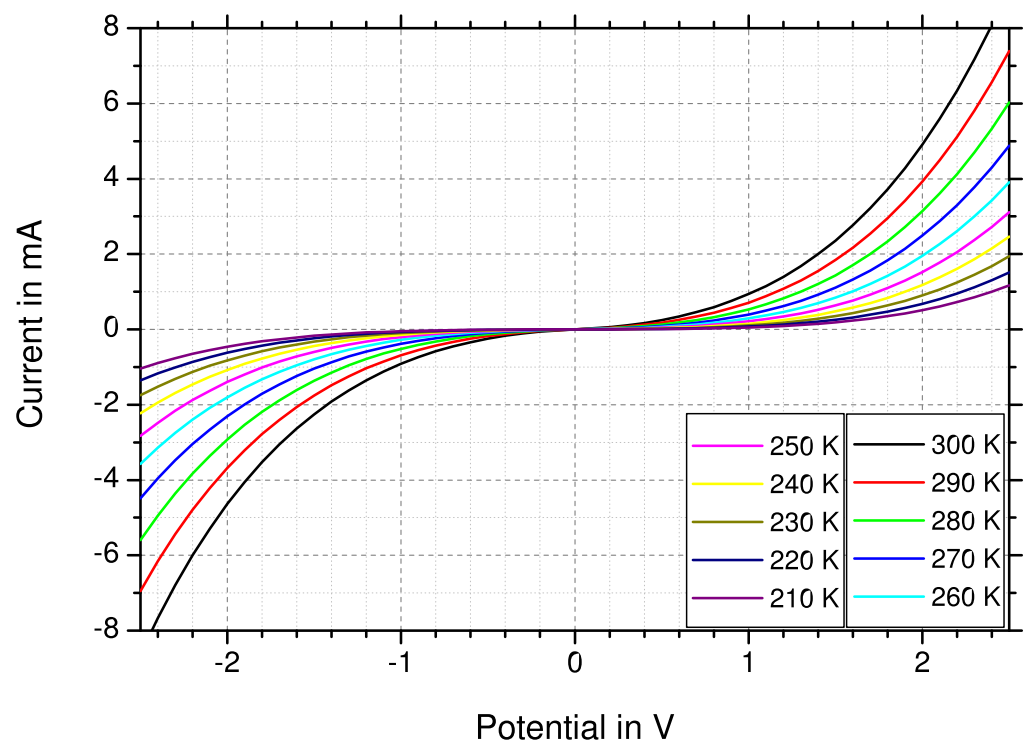

Figure 5.2: $I$ - $V$-characteristics measured on a virgin ta-C film at different temperatures $\left(6.37 \times 10^{-3} \mathrm{~cm}^{2}\right.$ contact and $85 \mathrm{~nm}$ film thickness $)$. All characteristics are similar in shape, mainly the overall conductivity increases with temperature. The symmetry of the characteristics indicates proper contacts with no significant Schottky effect between the chromium/gold-pad and the silicon substrate.

\subsection{Irradiation of ta-C}

Here, the general effect of ta-C irradiation is shown. As theoretically discussed above and shown in numerous publications, the ion bombardment will cause the formation of individual ion tracks [26, 28, 33, 80]. Analysis of individual tracks will be presented later in chapter 6. At this point the properties of track ensembles are presented. The pure ta-C was irradiated with $3 \times 10^{10} \mathrm{~cm}^{-2}$. The ion energy of the Uranium was $1 \mathrm{GeV}$ after being degraded by an aluminum foil. All pads on irradiated samples have a diameter of $0.6 \mathrm{~mm}\left(2.83 \times 10^{-3} \mathrm{~cm}^{2}\right)$. The contact covers about $8 \times 10^{7}$ tracks in parallel. The current measured is the result of a large ensemble of tracks conducting simultaneously. In parallel to the tracks, the unirradiated matrix is contacted as well. The following plot, figure 5.3, shows the sum of both currents at different potentials. In gray the currents of unirradiated ta- $\mathrm{C}$ is displayed (normalized to the contact area 
of $\left.2.83 \times 10^{-3} \mathrm{~cm}^{2}\right)$. Obviously, the conductivity increases by approximately three orders of magnitude by the irradiation process while only about $1 \%$ of the film area is effected by the irradiation. The increase in specific conductivity within the track volume is even larger than the three orders of magnitude shown by the data that leads to the conclusion of well pronounced conductive ion tracks embedded in an insulating matrix. In case of the previously presented unirradiated ta-C, the current increases with increasing temperature by seven orders of magnitude in the examined temperature range. In fact, the overall temperature dependence seems similar to the behavior of unirradiated ta-C.

At temperatures beyond $320 \mathrm{~K}$, the current limit of the bond wires is reached at $600 \mathrm{~mA}$. At this point the maximal field applied had to be reduced to keep within the specification. In order to minimize this limitation, all irradiated samples were contacted with the smallest pads available of $0.6 \mathrm{~mm}$ diameter.

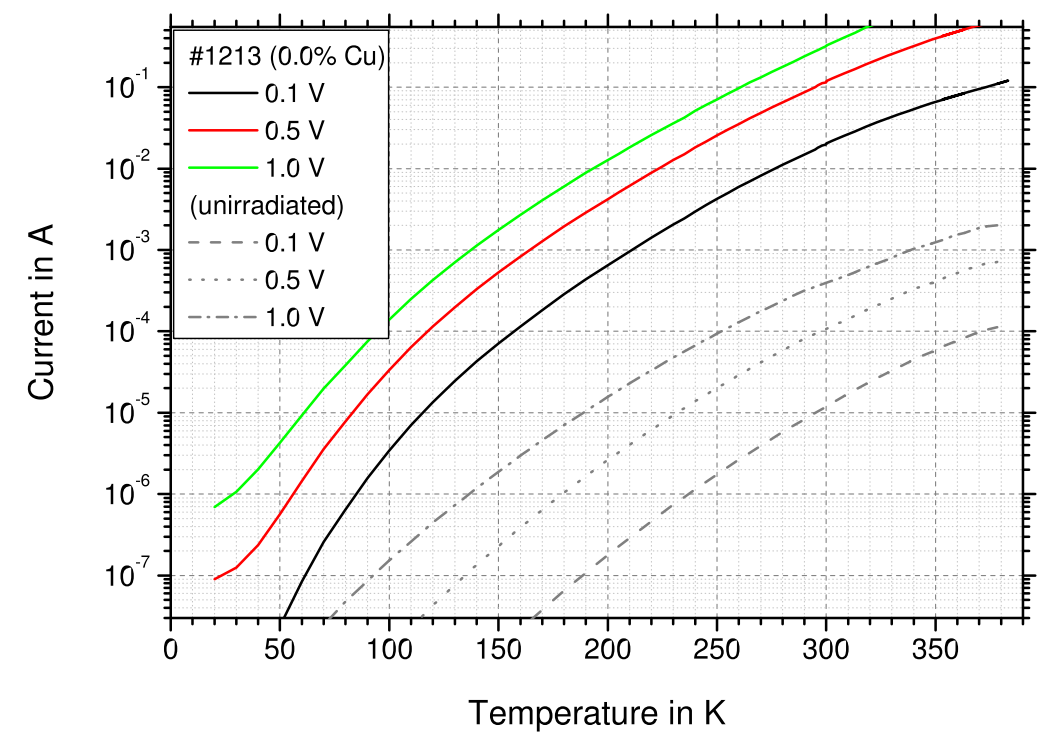

Figure 5.3: $I$-T-characteristics of different bias voltages measured on irradiated pure ta-C $\left(2.83 \times 10^{-3} \mathrm{~cm}^{2}\right.$ contact, $85 \mathrm{~nm}$ film thickness, $1 \mathrm{GeV} \mathrm{U}$ and a fluence of $\left.3 \times 10^{10} \mathrm{~cm}^{-2}\right)$. The contacted track ensembles makes up about $1 \%$ of the contact-area. Nonetheless, the conductivity increases by about three orders of magnitude compared to a similar unirradiated ta-C films. At low temperatures the residual conductivity is visible and at high temperatures the higher voltages reach at $600 \mathrm{~mA}$ the current limit of the setup, the characteristics are clipped.

In figure 5.4 some $I$ - $V$-characteristics are shown of the same irradiated film. The large increase in conductivity compared to unirradiated ta-C is obvious again. The characteristics look more ohmic and less s-shaped than the unirradiated counterparts. However, the maximal electrical field is smaller, a quantitative analysis of the shape is done later in chapter 5.6. Prior to this publication, ion track ensembles in 


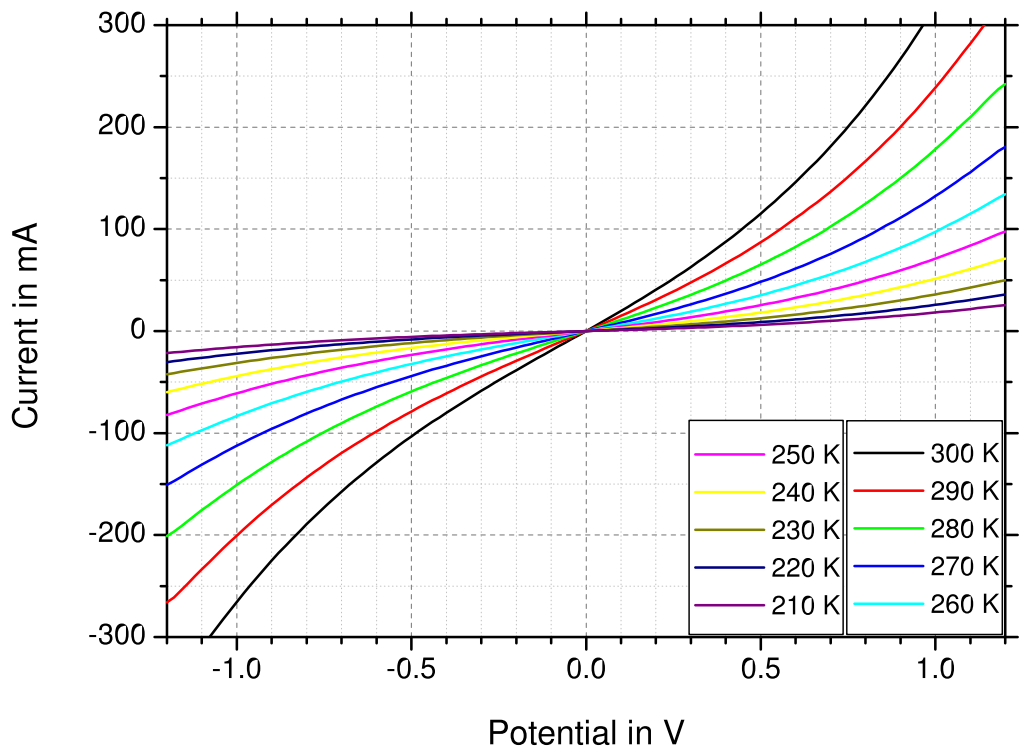

Figure 5.4: $I-V$-characteristics at different temperatures of the irradiated ta-C film shown in figure 5.3 All characteristics are s-shaped and similar to the characteristics of unirradiated ta-C. The curves show slightly asymmetric behavior caused by a degenerated Schottky contact of the metal contact pad and the silicon, which is exposed by the high current. The overall current is increased by a factor of 1000 compared to pristine ta-C .

comparable thin films were only measured to $0.3 \mathrm{~V}$. In this regime the characteristics seemed to be linear. Therefore, it was sometimes falsely suggested that ion track ensembles are ohmic at room temperature. The average resistance at room temperature of the track ensemble is about $5 \Omega$. This regime requires the remote setup to measure the behavior correctly. At these very low sample resistances, a small asymmetry of the characteristics is observed. At positive bias the characteristic is slightly rectifying. The asymmetry is field- and temperature-dependent; at $300 \mathrm{~K}$ it is about $20 \%$ at $1.2 \mathrm{~V}$ and $10 \%$ at $0.5 \mathrm{~V}$. At lower temperatures the values drop to $16 \%$ and $8 \%$ respectively. The most likely explanation of this behavior is a highly degenerated Schottky contact. The temperature dependence is less steep compared to the behavior of the ta- $\mathrm{C}$ tracks causing the effect to be more obvious at higher temperatures. The properties of the Schottky contact are dominated by the metal pad and the semiconducting silicon substrate and not by the ta-C, demonstrated in detail by Brötzmann 103 .

At low temperatures, the curvature of the characteristics increases and the nonohmic behavior is already visible at low fields. In figure 5.5 the regime below $100 \mathrm{~K}$ is displayed. In addition to the curvature, the steep temperature dependence becomes obvious. Between $100 \mathrm{~K}$ and $80 \mathrm{~K}$ the difference is about a factor three. At very low temperatures in the regime of $30 \mathrm{~K}$, the temperature dependence vanishes and the residual conductivity is dominant. 


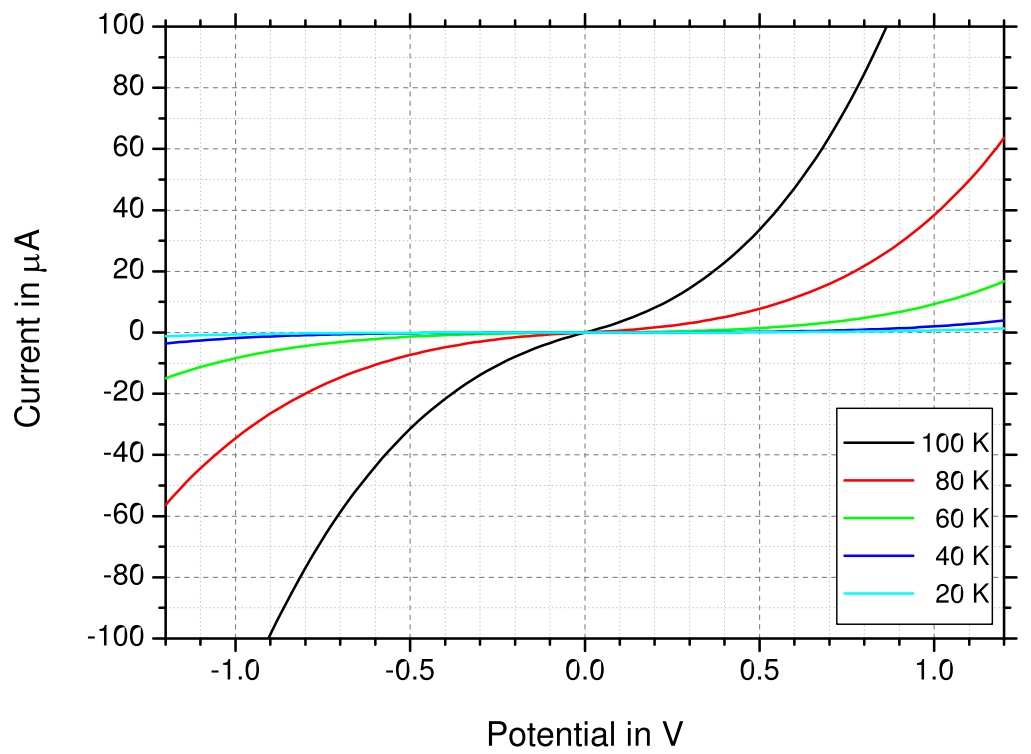

Figure 5.5: $I-V$-characteristics at different temperatures measured on irradiated ta-C (same sample as figure 5.3. The curvature is increased compared to room temperature. Approaching $0 \mathrm{~K}$, the curvature of the temperature independent residual conductivity becomes dominant as later shown in chapter 5.6 .

\subsection{Copper impurities in ta-C}

One major goal of this work is the analysis of the effect of copper impurities on the conductivity of ta-C and ion tacks. The effect of $\mathrm{B}$ and $\mathrm{N}$ on ta-C structure has been studied by Ronning et al. [164] as well as the effect on conductivity [35]. Nix and Krauser et al. examined the effect of $\mathrm{Fe}$ and $\mathrm{Cu}$ on ta-C as well as the effect on ion tracks [25, 36]. All these experiments have shown an increase of conductivity of the ta- $\mathrm{C}$ matrix and tracks. Nix has performed some benchmarking of the impurity effect comparing the increase of matrix and track conductivity. The most promising results were received by copper doped samples $(1 \% \mathrm{Cu})$. The ability of this copper concentration to retain a high contrast ratio of track and matrix conductivity has been noted by Gehrke [27] as well. In the following more extensive data on the effect of copper doping in ta-C will be presented.

So far, only concentrations of $1 \%$ at. were examined. In the scope of this work, the effect of different concentrations were tested ranging from $0.5 \%$ to $5 \%$. These samples were synthesized by depositing $\mathrm{x} \% \mathrm{Cu}$-ions and $100-\mathrm{x} \%$ carbon atoms quasi simultaneously (see chapter 3.1). Unintentionally, some ta-C samples supposed to be copper free show a small copper contamination. By XPS measurements the concentration was estimated to be less than $0.1 \%$. This concentration will be included in the following comparison. Table 5.1 provides an overview of the examined samples and their copper concentrations as deposited. 


\begin{tabular}{|c||c|c|c|c|}
\hline \# sample & $\begin{array}{c}\mathrm{Cu} \\
{[\%]}\end{array}$ & $\begin{array}{c}\text { thickness } \\
{[\mathrm{nm}]}\end{array}$ & $\begin{array}{c}\text { pad diameter } \\
{[\mathrm{cm}]}\end{array}$ & $\begin{array}{c}\text { fluence in } \\
{\left[10^{9}{\left.\text { ions } \mathrm{cm}^{-2}\right]}^{-1}\right.}\end{array}$ \\
\hline 1213 & 0 & 85 & 0.6 & 40 \\
1314 & 0 & 85 & 0.9 & 0 \\
\hline 1260 & 0.1 & 60 & 0.6 & 4 \\
\hline 1281 & 0.5 & 65 & 0.6 & 4 \\
\hline 1282 & 1 & 65 & 0.6 & 4 \\
\hline 1279 & 2 & 65 & 0.6 & 4 \\
\hline 1283 & 4 & 45 & 0.6 & 4 \\
\hline
\end{tabular}

Table 5.1: Tabular overview of ta-C samples used in following examinations. Every sample, except \#1213 and \#1314, was divided into several pieces. One part of each sample was not irradiated and kept as reference. Since both matrix and track data were measured on the same sample, the comparability should be as high as possible. All irradiations were performed with $1 \mathrm{GeV} \mathrm{U}$ at GSI.

In figure 5.6 the temperature-dependent current of sample \#1260 is displayed for three different voltages. In comparison the data of pure ta-C is shown in gray. The difference of the contact pad area was normalized, the difference in sample thickness was neglected. The conductivity increases by about a factor of 20 . The overall temperature dependence is similar. Towards lower temperatures the conductivity approaches the values of the pure ta-C and again at extremely low temperatures around $20 \mathrm{~K}$ a residual conductivity is dominant. At $300 \mathrm{~K}$ a kink is visible where the low- and high-temperature measurement were merged. This is a measurement artifact and not part of the sample behavior. The $I-V$-characteristics shown in figure 5.7 are similar in shape compared to pure ta-C. The overall conductivity is larger and the curves are again almost symmetric.

In the next step, figure 5.8 shows the current measurement on an irradiated ta-C film containing less than $0.1 \% \mathrm{Cu}$. In gray, the measurement of the unirradiated $0.1 \%$ - $\mathrm{Cu}$ film is shown in comparison. The conductivity increases over the complete temperature range. Again, the overall temperature dependence is similar to the measurements shown before on pure ta-C. The residual conductivity is visible below $30 \mathrm{~K}$ also.

The difference of matrix and track conductivity seems much smaller than in the case of pure ta-C (figure 5.3). However, it should be noted that the irradiation fluence is one order of magnitude smaller. Figure 5.9 shows the characteristics of the irradiated samples with $0.1 \% \mathrm{Cu}$. The main difference to pure ta- $\mathrm{C}$ is the smaller curvature exhibited. At room temperature, the characteristics are almost ohmic up to a bias of $1 \mathrm{~V}$. The rectification of the curves is seen in a similar manner as in the case of pure ta-C.

The temperature dependence and the shape of the characteristics up to $2 \% \mathrm{Cu}$ are comparable to the results of \#1260, mainly the conductivity increases with $\mathrm{Cu}$ concentration and irradiation. 


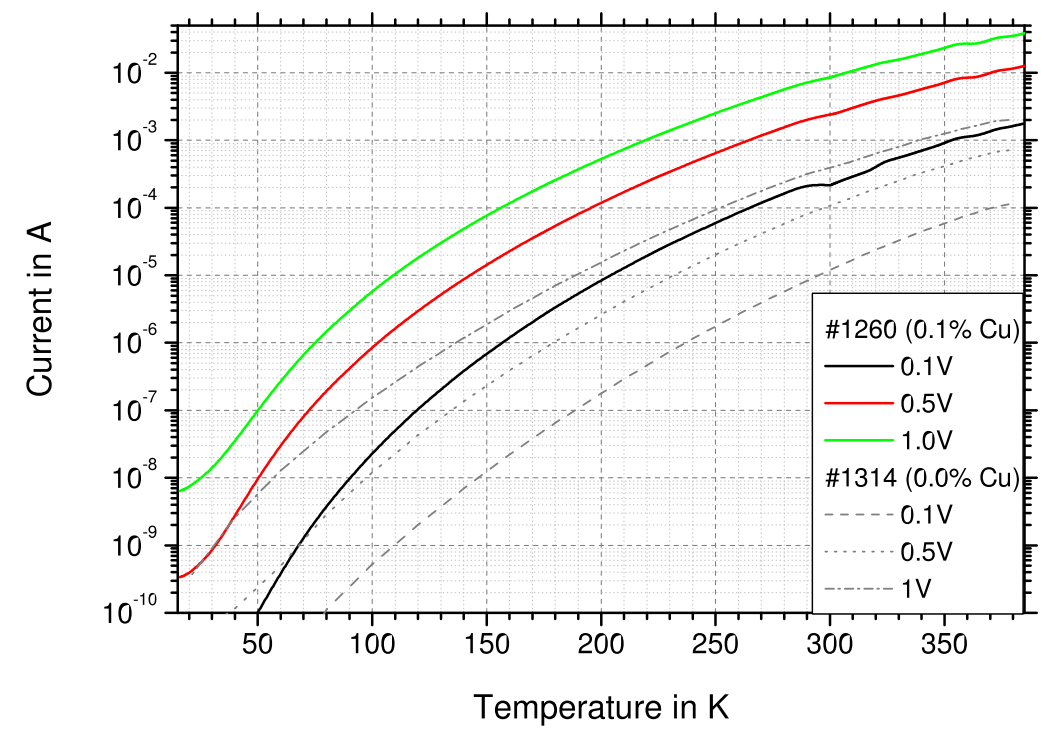

Figure 5.6: $I-T$-characteristics of unirradiated ta-C:Cu with about $0.1 \% \mathrm{Cu}$ at different bias potentials. In gray the respective characteristics of the pure ta-C are shown. The conductivity is increased by about a factor of 20 . At low temperatures, a temperature independent conductivity is suggested as in case of pure ta-C.

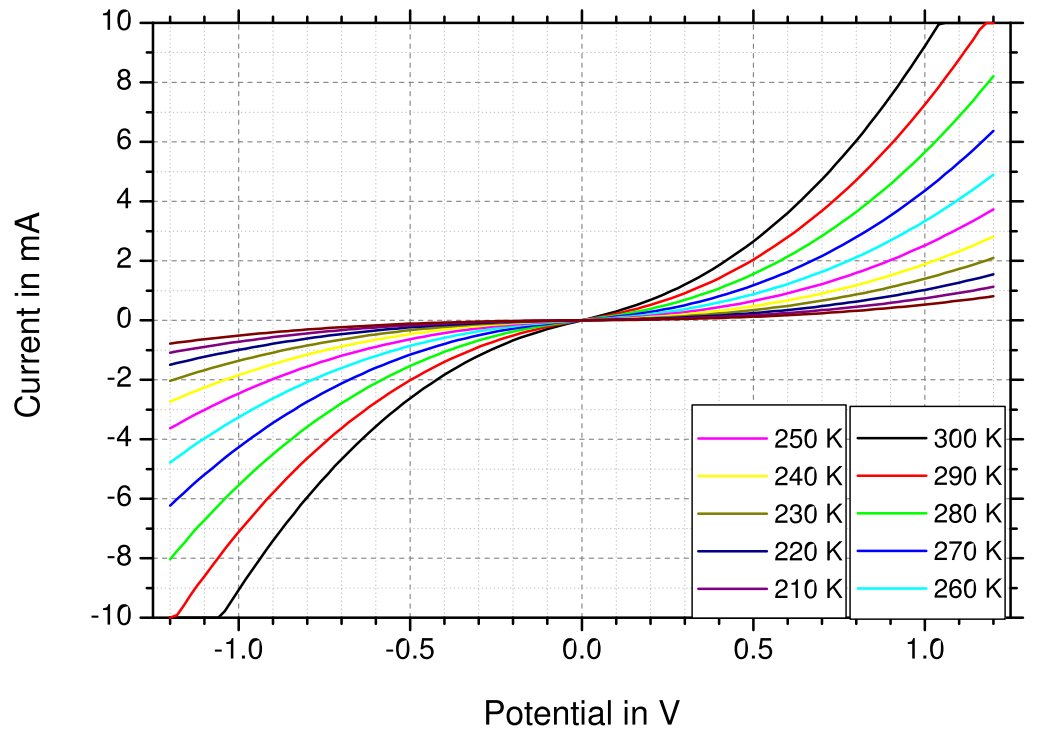

Figure 5.7: $I-V$-characteristics of ta-C:Cu with about $0.1 \% \mathrm{Cu}$ content (\#1260). In case of pure ta-C , the characteristics are symmetric and s-shaped. The overall conductivity is larger as expected for ta-C:Cu films. 


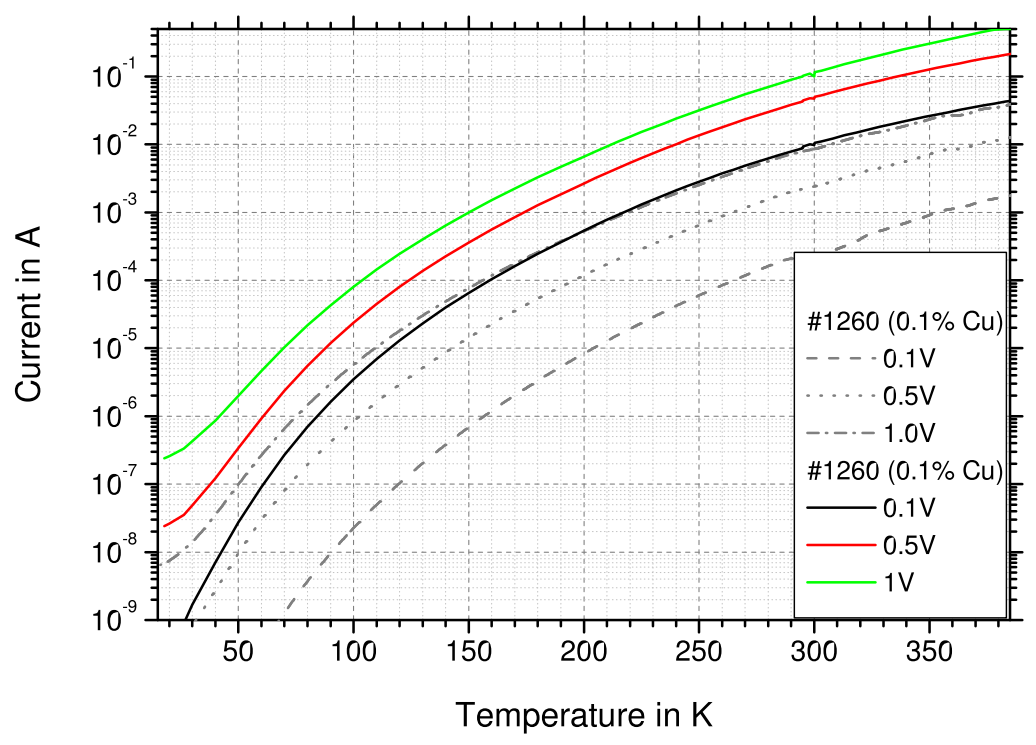

Figure 5.8: $I$ - $T$-characteristics of irradiated ta-C:Cu with about $0.1 \% \mathrm{Cu}$ content (\#1260). In comparison, the data of the unirradiated sample is shown in gray. The increase by irradiation is smaller than in case of pure ta-C. However, the effect is exaggerated by the lower ion fluence used for the doped samples. At low temperatures, the usual residual conductivity is visible.

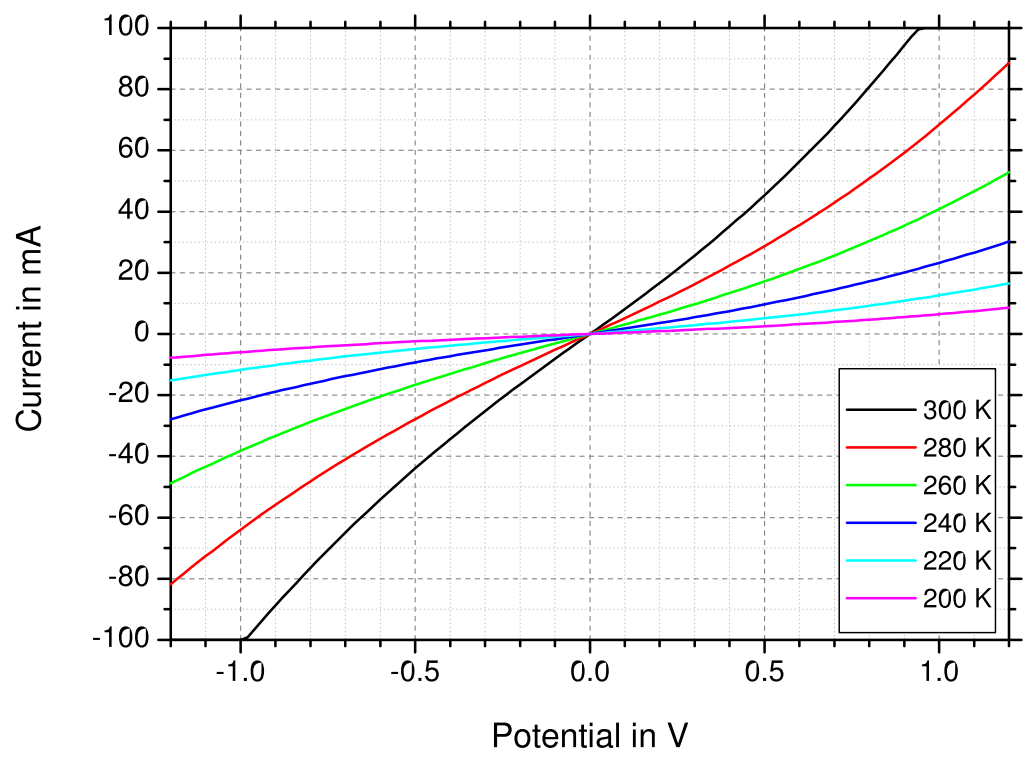

Figure 5.9: $I-V$-characteristics measured of irradiated ta-C:Cu with about $0.1 \% \mathrm{Cu}$ content (\#1260). Besides the increased conductivity compared to pure ta-C and unirradiated doped films, although the curvature is decreased. At room temperature, the behavior is ohmic up to the applied field. 
At $5 \% \mathrm{Cu}$, the highest concentration, the results differ from the results of pure ta- $\mathrm{C}$ and ta-C with smaller amounts of impurities. The $\mathrm{Cu}$ changes the $\mathrm{sp}^{3}$ hybridization of the ta-C as shown by XPS spectroscopy (chapter 4 ) slightly. The bond ratio of the film changes to a more graphitic structure with higher $\mathrm{sp}^{2}$ content. In figure 5.10, the data of the $5 \% \mathrm{Cu}$ sample is shown in comparison to unirradiated pure ta-C . The increase in conductivity, caused by $5 \%$ copper, is quite large, even before irradiation. The changed matrix has a conductivity almost as high as the irradiated pure sample. In turn, the irradiation process does not increase the conductivity as much as seen in samples with smaller $\mathrm{Cu}$ concentration.

The temperature dependence of the $5 \% \mathrm{Cu}$ sample is still similar to pure ta-C suggesting unchanged conduction mechanisms. The current measured decreases by many orders of magnitude towards lower temperatures and below $50 \mathrm{~K}$, the residual conductivity is visible. However, compared to lower $\mathrm{Cu}$ concentration, the shape of the $I-V$-characteristics appears much more ohmic in the examined bias range. Towards smaller temperatures, the curvature increases as suggested in figure 5.11. showing a selection of characteristics of the unirradiated sample. The characteristics are almost symmetric, a slight rectifying behavior is observed as seen in other irradiated ta-C samples. The irradiation increases the conductivity only by a factor of two. The shape of the characteristics does not change significantly by irradiation as seen in figure 5.12. The rectifying property is more obvious at these higher currents.

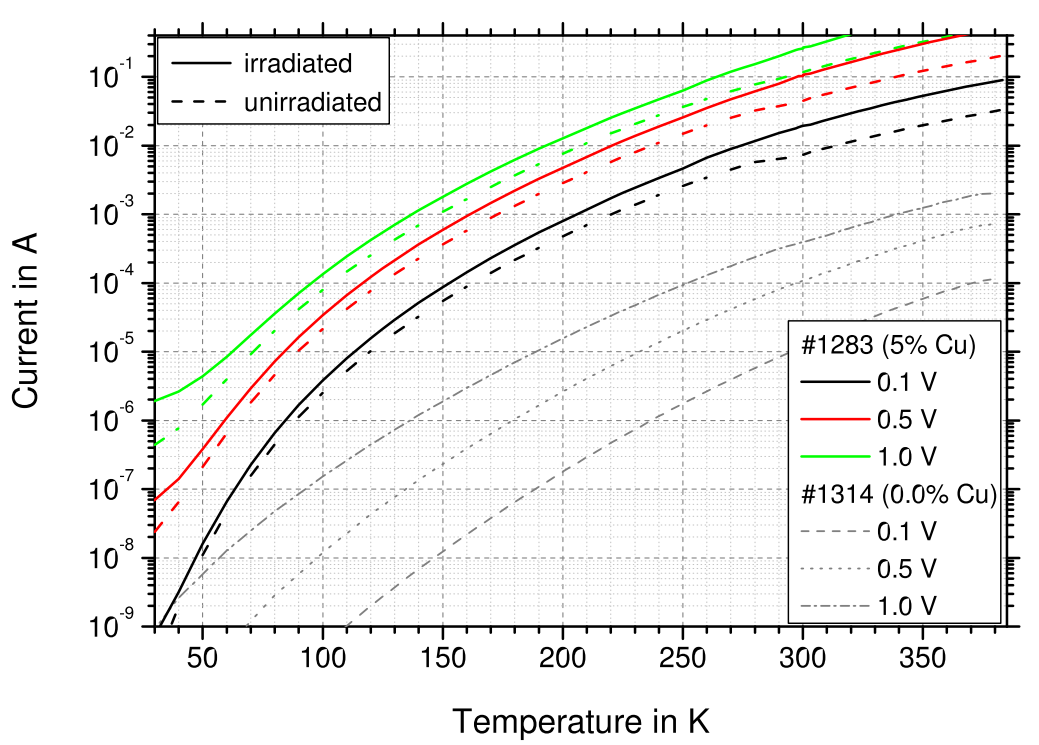

Figure 5.10: $I-T$-characteristics of ta-C:Cu with about $5 \% \mathrm{Cu}$ content. In gray the respective characteristics of the pure ta-C are shown. The doped matrix reaches almost the same current as seen after irradiation. The difference is only about a factor of two. On the other hand, the temperature dependence is still comparable to pure ta-C. 


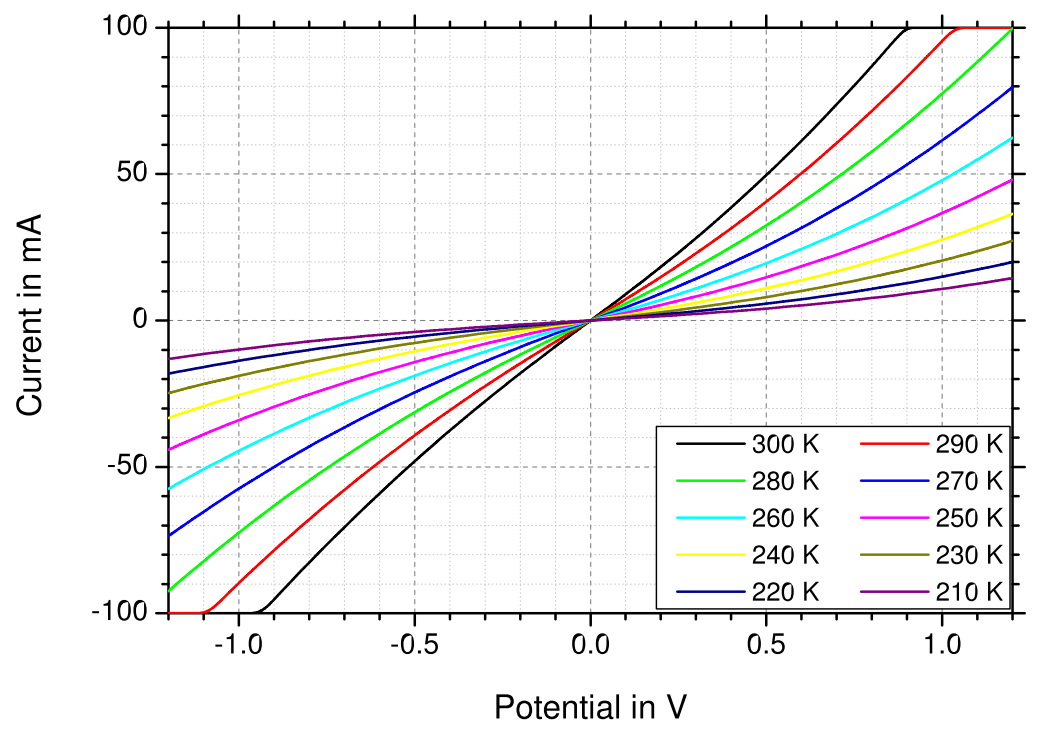

Figure 5.11: $I-V$-characteristics of ta-C:Cu $(5 \% \mathrm{Cu})$. The curves are almost ohmic at room temperature in the bias regime analyzed. Towards lower temperatures, the curvature increases. The measurements exhibit almost symmetrical behavior. Since the current is in the order of irradiated samples shown before, a slight rectifying property is already seen in this unirradiated film.

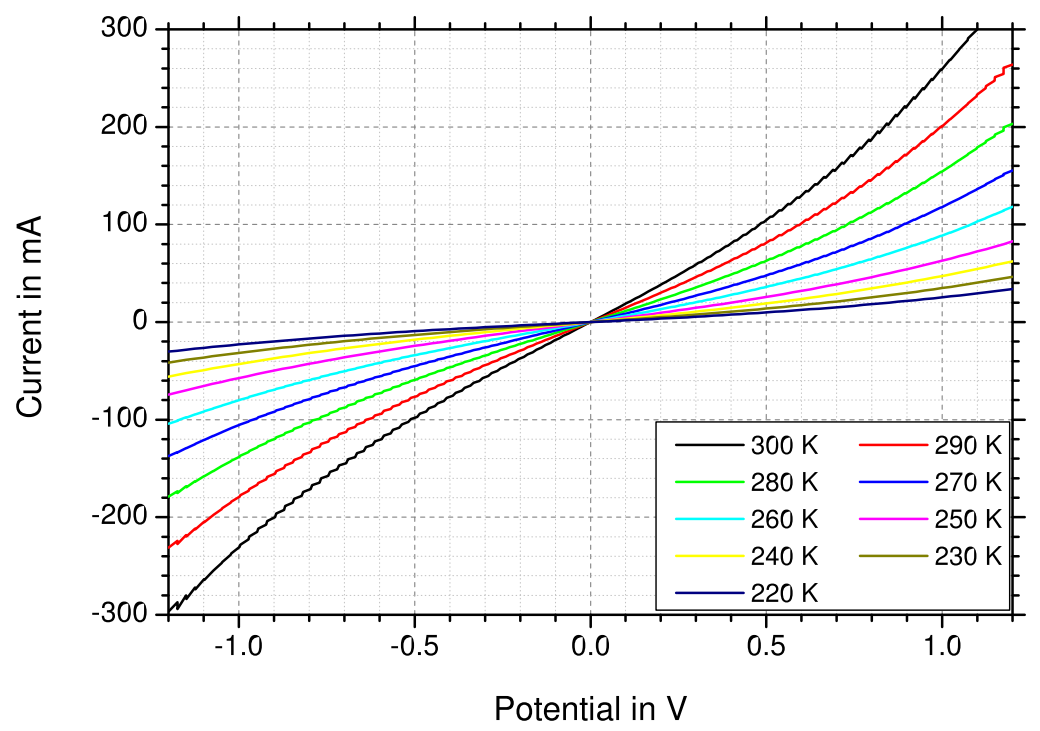

Figure 5.12: $I-V$-characteristics of irradiated ta-C: $\mathrm{Cu}(5 \%)$. The current has increased by a factor of about two by irradiation. At room temperature, the curvature is small but increases toward lower temperatures. As before, if high currents flow, the behavior is slightly asymmetrical because of the degenerated Schottky-contact formed. 


\subsection{Comparison of $I-V$-characteristics of ta- $\mathrm{C}: \mathrm{Cu}$}

To visualize the effect of the copper, the $I-V$-characteristics of all films are compared at different temperatures: 2 ;

Figure 5.13(a) displays the logarithm of the current for positive bias at $373 \mathrm{~K}$. In this comparison, the effect of copper on the matrix can be quantitatively seen. The sample with $5 \% \mathrm{Cu}$ has a current exceeding the pure ta-C film by a factor of 250 at $0.5 \mathrm{~V}$. A copper concentration of $2 \%$ increases the current at the same potential by a factor of 50, at higher voltages even by 75 . The smallest amount of about $0.1 \%$ $\mathrm{Cu}$ increases the current by a factor of 16 .

At $300 \mathrm{~K}$, the overall picture is very similar to measurements at $373 \mathrm{~K}$, only the relative gain in current, caused by $\mathrm{Cu}$, is larger at room temperature. As seen in figure $5.13(\mathrm{~b})$, the difference of pure ta-C and $5 \% \mathrm{Cu}$ has almost doubled to a factor of 456. Towards smaller copper concentrations, the raise in current is about $20 \%$. However, at high fields, the factor for pure ta-C and $2 \% \mathrm{Cu}$ has decreased by $25 \%$ compared to high-temperature characteristics being an indicator for different temperature dependence of the conduction mechanisms at low and high fields.

The data at lower temperatures (200 K in figure 5.13(c) ) continues the trend. The factor for $5 \% \mathrm{Cu}$ to pure ta- $\mathrm{C}$ has increased to 1080 . The ratio has doubled in comparison to room temperature. The ratio of the currents for $0 \%$ and $0.1 \% \mathrm{Cu}$ doped samples has increased to 45 about $90 \%$ more than at room temperature. The difference between $0.1 \%$ and $5 \%$ is more stable increasing only by $25 \%$. At high fields, the ratio is almost constant between $0 \%$ and $2 \%$ showing a different correlation for the conductivity at low and high fields.

Finally, at low temperatures around $100 \mathrm{~K}$ (figure $5.13(\mathrm{~d})$ ), the trend at $0.5 \mathrm{~V}$ still continues. The factor between pure ta- $\mathrm{C}$ and the doped film with $5 \% \mathrm{Cu}$ shows a ratio of 1775 that is an increase of $64 \%$ compared to the ratio at $200 \mathrm{~K}$. Between $0 \%$ and $0.1 \%$ the ratio increased by $58 \%$ in the same temperature range. Between higher copper concentrations, the change in current ratio is stable at low temperatures. The difference at $200 \mathrm{~K}$ and $100 \mathrm{~K}$ is only $4 \%$. At high fields, the current ratio of pure and $2 \% \mathrm{Cu}$-doped ta-C is about 30 to 40 if the $I$ - $V$-characteristic is extrapolated.

This simple comparison of $I$ - $V$-characteristics leads to a few qualitative conclusions. The effect of $\mathrm{Cu}$ doping is most apparent at low fields (more ohmic behavior with increasing copper concentration). Furthermore, the effect of $\mathrm{Cu}$ is more dominant at low temperatures while hardly affecting the temperature dependence. In order to further quantify the effect of copper on the ta-C matrix and tracks and to understand the mechanisms causing the conductivity of the different films, a fitting model is developed in the following sections.

\footnotetext{
${ }^{2}$ The data of the current is comparable because the contact-area on each sample was the same. $1 \mathrm{~mA}$ is equivalent to about $3.53 \times 10^{3} \mathrm{~A} \mathrm{~m}^{-2}$
} 


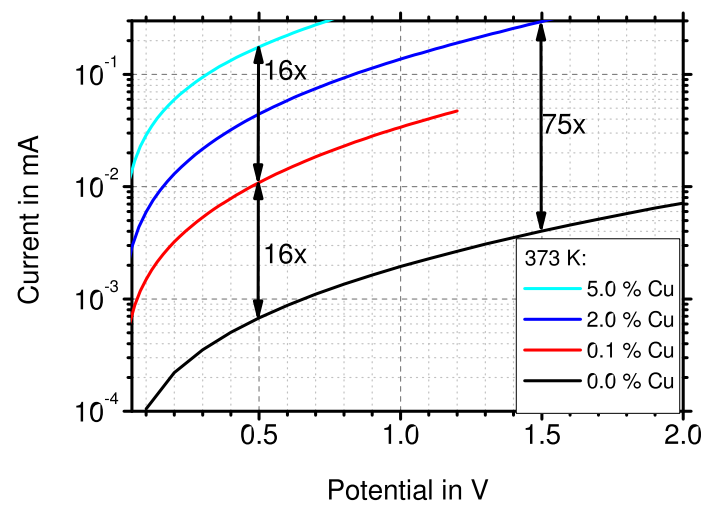

(a) Comparison at $373 \mathrm{~K}$

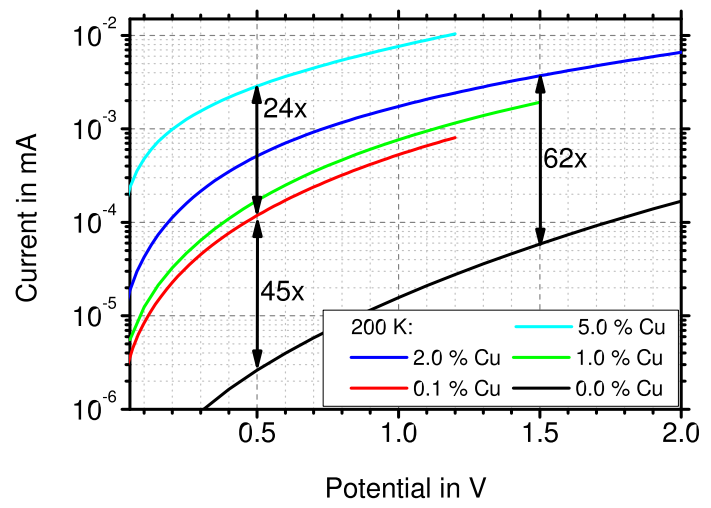

(c) Comparison at $200 \mathrm{~K}$

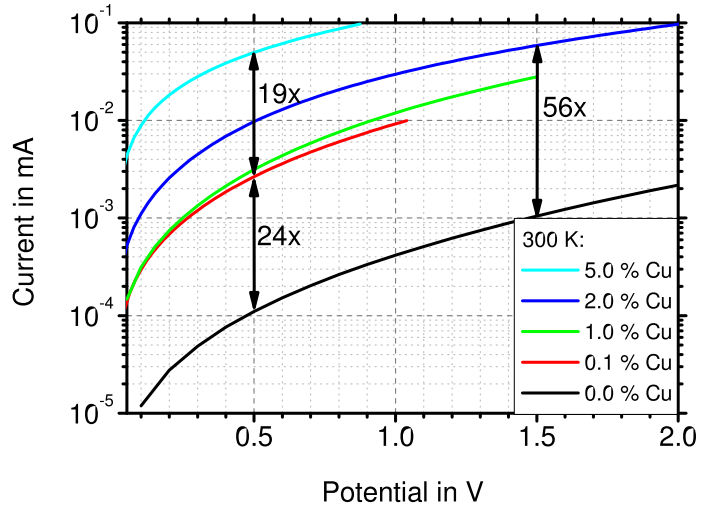

(b) Comparison at $300 \mathrm{~K}$

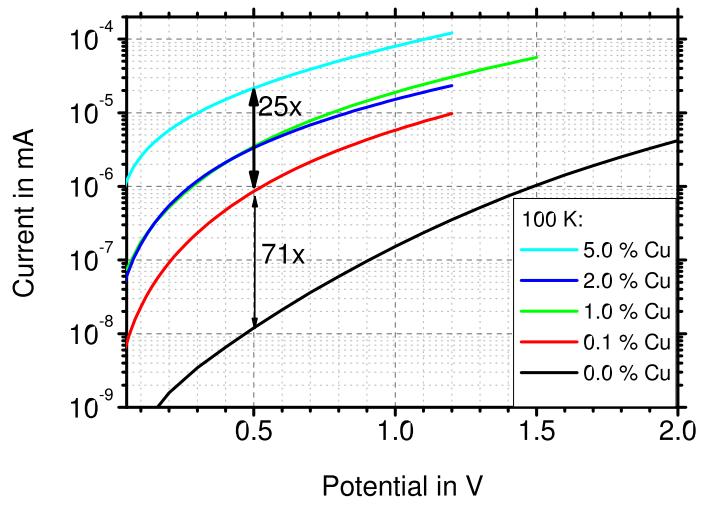

(d) Comparison at $100 \mathrm{~K}$

Figure 5.13: $I$ - $V$-characteristics of unirradiated ta-C and ta-C: $\mathrm{Cu}$ at different temperatures. The difference in conductivity is shown as a factor for some points. At all temperatures, the conductivity increases with the copper content. The ratios vary with temperature and applied field. The effect of copper is largest at low temperatures and low bias voltages.

\subsection{Frenkel-Poole model for virgin ta-C}

Already Nix [32, 33] and Schwen [22] suggested a Frenkel-Poole fit to describe the $I-V$-T-behavior of ta-C close to room temperature. The sole application of the Frenkel-Poole (FP) formula did not yield satisfying predictions for the electrical behavior at different temperatures and low fields. The curvature was overestimated and the conductivity at low temperatures was underestimated. An extension to the FP model was developed by Brötzmann and Gehrke (published by Brötzmann et al. [170]) to describe the behavior of ta-C with the goal to fit the characteristics more precisely at low and high fields and to cover a larger temperature range 
while keeping a simplistic model. Brötzmann used this FP extension successfully for the description of ta-C and $\mathrm{BN}$ in his model for metal-amorphous-semiconductorsemiconductor systems 103,171 .

The difference to the description of Nix and Schwens is a parallel ohmic resistor to the FP mechanism. The current density is equivalent to

$$
J(F, T)=\sigma_{\mathrm{p}}(T) F+\tilde{\sigma}_{\mathrm{fp}}(F, T) F,
$$

where $\sigma_{\mathrm{p}}$ is the temperature-dependent conductivity of the added parallel resistor, $\tilde{\sigma}_{\mathrm{fp}}=\sigma_{\mathrm{fp}} \cdot \exp \left(-\frac{q \phi_{\mathrm{fp}}}{k_{\mathrm{B}} T}\right)$ is the temperature and field-dependent conductivity of the FP equation and $F=\frac{U}{d}$ is the electric field. The physical argument for the addition of the parasitical resistor can be found in the failure of the FP model in case of barrierexhaustion, if more than one level is present, and in case of tunneling (chapter 2.5.1). Here, the ohmic resistor is a first-order approximation for these effects.

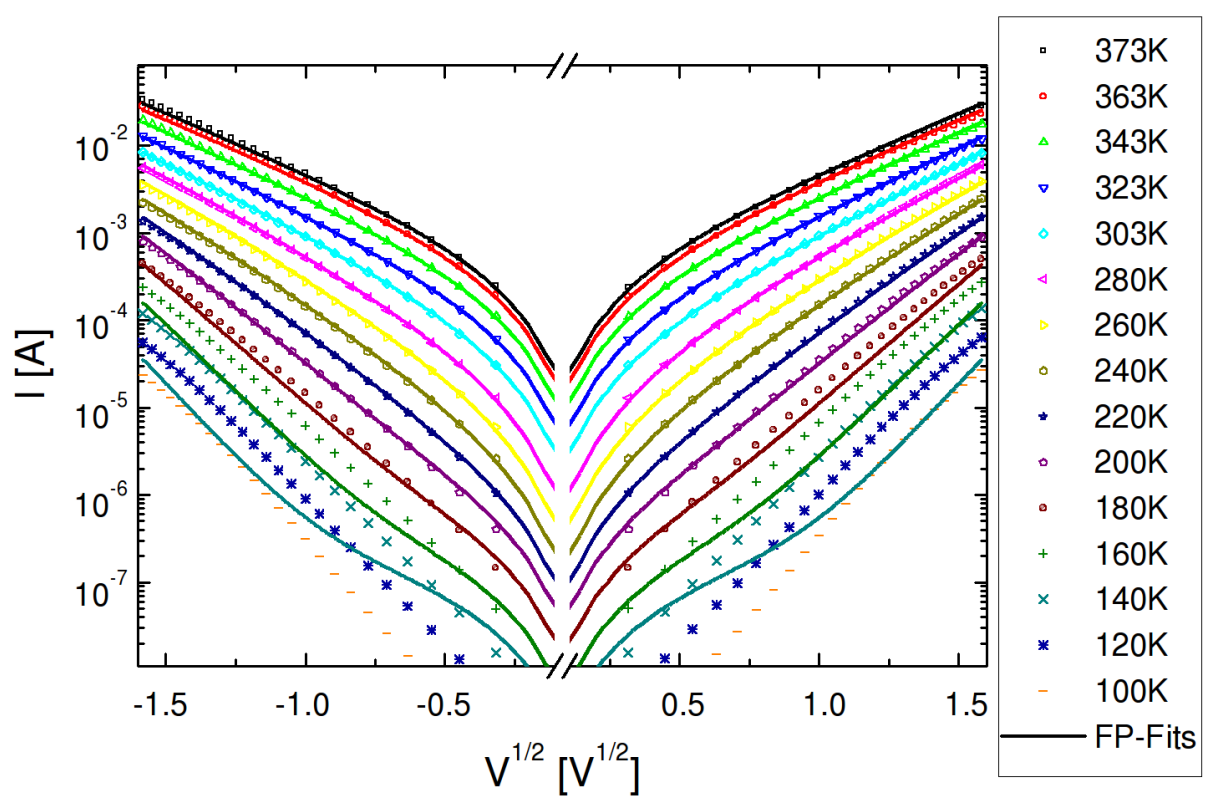

Figure 5.14: $I$ - $V$-characteristics and extended-FP fits for temperature-dependent data measured on virgin ta-C. The extended-FP model is valid for the temperature range of $200 \mathrm{~K}$ to $373 \mathrm{~K}$. At lower temperatures, the fit underestimates the measured current.

As shown in figure 5.14 this extended FP model describes the characteristics of ta-C well between $200 \mathrm{~K}$ to $373 \mathrm{~K}$. To successfully obtain reliable values for all free parameters of the model, the following procedure was used. First, the temperature dependence was fitted to obtain a stable value for $\phi_{\mathrm{fp}}$ as defined in equation 2.29 which was accomplish by examining the logarithm of the FP formula:

$$
\ln (J(F, T))=\ln \left(\sigma_{\mathrm{fp}} F\right)+\left(-\frac{q \phi_{\mathrm{fp}}}{k_{\mathrm{B}} T}\right)+\left(\frac{1}{k_{\mathrm{B}} T} \cdot \sqrt{\frac{q^{3} F}{\pi \epsilon \epsilon_{0}}}\right) .
$$


At high fields, the logarithm of the current is proportional to the square-root of the field $\sqrt{F}$, neglecting the first term. Equation 5.2 is now a linear function of $\sqrt{F}$, that can be extrapolated to $F=0$. The y-intercept of this function is equivalent to:

$$
y(T)=c+\left(-\frac{q \phi_{\mathrm{fp}}}{k_{\mathrm{B}} T}\right)
$$

where $c$ is a temperature independent constant. Equation 5.3 is derived by $\frac{1}{T}$ :

$$
\frac{\mathrm{d} \mathrm{y}(\mathrm{T})}{\mathrm{d} \frac{1}{T}}=-\frac{q \phi_{\mathrm{fp}}}{k_{\mathrm{B}}} .
$$

Numerically, the y-intercept $y(T)$ was calculated of two different empirical characteristics at adjacent temperatures (1 and 2) by extrapolating the high-field slope of $\sqrt{F}$. The barrier height can be calculated by solving equation 5.4 entering the values obtained by experiment:

$$
\phi_{\mathrm{fp}}=-\frac{k_{\mathrm{B}}}{q} \cdot \ln \left(\frac{y_{1}\left(T_{1}\right)}{y_{2}\left(T_{2}\right)}\right) \cdot\left(\frac{T_{1} T_{2}}{T_{2}-T_{1}}\right) .
$$

This method depends on the $\sqrt{F}$ dependence of $\ln (I)$, which requires the examination of sufficiently high fields. In case of the ta-C samples shown here, at least $1.5 \mathrm{~V}$ $\left(2 \times 10^{7} \mathrm{~V} \mathrm{~m}^{-1}\right)$ should be applied. Secondly, the ohmic contribution $\sigma_{\mathrm{p}}$ must be comparatively small at the fields used for extrapolation. Both requirements were fulfilled and a value of $\phi_{\mathrm{fp}}=0.25 \mathrm{~V}$ was calculated for ta- $\mathrm{C}$ which is identical to the results of Brötzmann 103.

In the second step of the fitting procedure the parallel resistance, $\sigma_{\mathrm{p}}$, and $\epsilon$ were fitted with a least square method programmed in python v2.7 [172] using scipy [173]. In figure 5.14 the logarithm of the current is plotted versus $\sqrt{F}$ (Schottky-Plot) in order to visualize the FP behavior at high fields. The results of the fit are compared to the measured data. For potentials exceeding $1 \mathrm{~V}$, the characteristics become linear in this representation of the data, as expected for FP conduction. In the temperature range of $200 \mathrm{~K}$ to $373 \mathrm{~K}$ the fit is very precise and the data is well modeled. Towards lower temperatures, the fit overestimate the data. In addition, the slope varies with temperature at high fields, which is caused by assuming a constant effective barrier height $\phi_{\mathrm{fp}}$ compare to chapter 2.5.1.

The current, described by the FP model, depends exponentially on temperature and field. In other words, if the bias voltage is constant, the film thickness has an exponential impact on conductivity. This behavior is significantly different to interface effects such as Schottky emission, which shows similar field dependence but is less dependent on film thickness. The thickness dependence of the characteristics of ta-C films was measured on different contact pads on a film with a gradient in film thickness. Ten characteristics were recorded at different sample positions using 
pads with a diameter of $0.9 \mathrm{~mm}$ at $297 \mathrm{~K}$. In figure 5.15(a) a selection of different characteristics is displayed. The s-shaped nature of the curves and the overall current are comparable with the data shown in figure 5.2. To relate the data to the film thickness, the $I$ - $V$-characteristics were fitted using a numerically simplified form of the Frenkel Poole formula (equation 2.29):

$$
I=\frac{V}{d_{\mathrm{fit}}} \cdot \exp \left[b\left(\frac{V}{d_{\mathrm{fit}}}\right)^{0.5}\right],
$$

where $b=450 \mathrm{~m}^{1 / 2} \mathrm{~V}^{-1 / 2}, V$ is the potential, and $d$ the fit parameter for film thickness. The advantage of this formula compared to the original FP model is the combination of curvature and thermal dependence into one parameter $b$. In addition, the parasitic ohmic resistor of the extended FP model is included in $b$ as well.

First, both $b$ and $d$ were set as free parameters and each curve was fitted. The curvature $b$ varies for the different characteristics from $350 \mathrm{~m}^{1 / 2} \mathrm{~V}^{-1 / 2}$ to $500 \mathrm{~m}^{1 / 2} \mathrm{~V}^{-1 / 2}$. To receive stable results for $d$, the curvature was fixed to the weighted average value of $450 \mathrm{~m}^{1 / 2} \mathrm{~V}^{-1 / 2}$. The resulting values for $d$ were normalized by a common factor of 521 to correspond to the empirical thickness values. The empirical estimation of the thickness was done by the color of the thin-film interference. This procedure was calibrated by comparing the colors to the colors of samples analyzed by TEM providing exact measurements of film thickness. In addition, the average film thickness is known from the deposited amount of $\mathrm{C}^{+}$ions. The margin of systematic errors was relatively high since both sets of values for $d$ had to be calibrated differently. However, the trend of each set is reliable. The results are shown in figure $5.15(\mathrm{~b})$. The trend of the fitted data corresponds well with the measured values supporting the validly of a thickness-dependent bulk mechanism in these ta-C films instead of interface governed processes such as Schottky-emission.

Besides supporting the validity of the FP model for ta-C, this experiment shows the strong dependence of the conductivity on film thickness. Any calculated values for conductivity, which were extracted from measured currents, strongly depend on well known film thicknesses. An uncertainty of $10 \%$ in film thickness results in an uncertainty of $25 \%$ for conductivities calculated from currents using the FP model. The typical variation in film thickness of samples used in this work can be estimated by $10 \%$. 


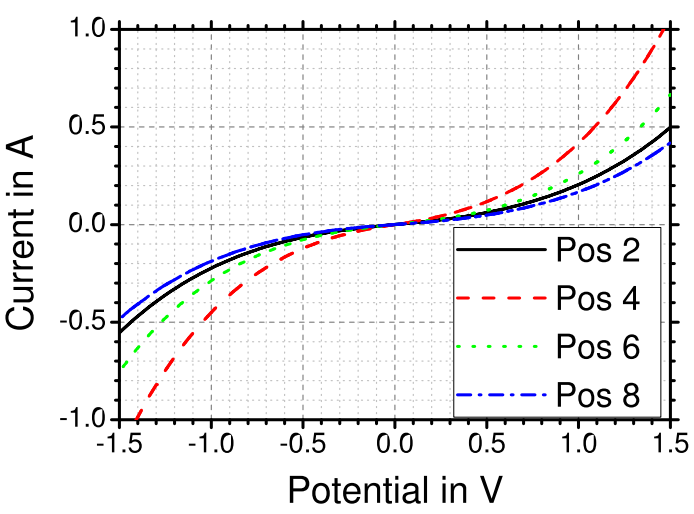

(a) $I$ - $V$-characteristics at different positions

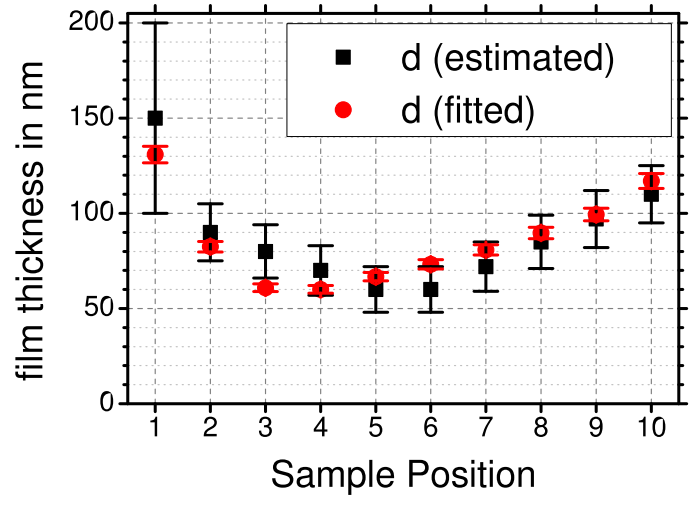

(b) Comparison of fitted and estimated $d$

Figure 5.15: Correlation of ta-C film thickness $d$ and conductivity. (a) shows four selected characteristics measured at different contact pads with different film thickness. All pads with identical dimensions were on the same sample, only the thickness varies due to a gradient in this particular sample. (b) compares the film thickness simulated by FP fit and the estimated film thickness by color resulting from thin film interference. The conductivity of the ta- $\mathrm{C}$ films are thickness-dependent and correlate well with the trend predicted by FP mechanism

\subsection{Development of an empirical model for $I-V$ - $T$-behavior}

The extension of the Frenkel-Poole model works well for pure ta-C down to $200 \mathrm{~K}$. The addition of the parasitic ohmic resistor is even capable to describe the behavior of irradiated ta-C and ta-C:Cu films to some extent. However, the model cannot describe the behavior at low temperatures and is limited to low ohmic contributions. Furthermore, the assumption of fixed curvatures (constant $\epsilon$ and $\beta_{\mathrm{fp}}$ ) cannot be accomplished without assuming non-physical values for $\epsilon$, especially at high copper concentrations or after irradiation. As seen by the above fit in figure 5.14 at least one other conduction mechanism should be dominant at low temperatures, which needs to be added to the model. One candidate is hopping, in particular VRH. The parallel contribution of hopping and FP is common in amorphous thin films. For example, Simmons has reviewed different thin-film-systems exhibiting more than one mechanism 106.

Ultimately, each conduction mechanism has two main dependencies: temperature and field. The field dependency can be removed by observing conductivity close to zero-bias. Here, the FP mechanism is reduced to the simple thermal activation. A major draw-back of the above procedure to fit $\phi_{\mathrm{fp}}$ is the inability to separate field and thermal dependence of the conductivity; both have to be fitted simultaneously (see chapter 5.5).

To overcome this problem, a different approach was taken. The characteristics were fitted with a mathematical function separating thermal and field dependence. 
At the same time, the function was capable to simulate the curvature of the sshaped characteristics as well as ohmic behavior observed in the presence of tracks and high $\mathrm{Cu}$ concentrations. The total parameter-space was reduced to three scalar positive variables, one for the temperature dependence and two for the curvature. The mathematical function fulfilling the requirements is the stretched exponential function:

$$
I=\sigma_{0} \frac{V A}{d} \exp \left[\left(\frac{\beta V}{d}\right)^{\gamma}\right],
$$

where $A$ is the contact area, $d$ the film thickness, $\sigma_{0}, \beta$ and $\gamma$ are fitting parameter. This function is equivalent to the s-shaped FP model assuming $\sigma_{0}=\sigma_{\mathrm{fp}} \cdot \exp \left(-\frac{q \phi_{\mathrm{pp}}}{k_{\mathrm{B}} T}\right)$, $\beta=\frac{q^{3}}{\pi \epsilon \epsilon_{0}}$ and $\gamma=\frac{1}{2}$. Ohmic behavior is modeled by $\sigma_{0}$ as $\beta$ approaches zero. This function is capable to fit linear- and s-shaped characteristics. The temperature dependence of the zero-bias conductivity is represented by $\sigma_{0}$. A distinction in barrier height $\left(\phi_{\mathrm{fp}}\right)$ and proportional factor $\left(\sigma_{\mathrm{fp}}\right)$, as done in the FP model, is not necessary.

The exponent $\gamma$ describes the type of curvature. Physically common models for field dependence of the different conduction models vary. Here, the parameter is restricted to values between $0.5(\mathrm{FP})$ and 1 (moderate-field correction in VRH). The parameter $\beta$ represents the amount of curvature relative to the zero-bias conductivity.

The contact area for the fitting-procedure of the unirradiated ta-C samples $\left(A_{\mathrm{u}}\right)$ is equivalent to the surface-area of the $\mathrm{Cr} / \mathrm{Au} \mathrm{pad}$ evaporated onto the film. The film thickness was taken from deposition data, which had been calibrated by TEM analysis. In case of the irradiated samples, only the track conductivity should be fitted. The contact area is assumed to be equivalent to the area effectively changed by the irradiation (track area). The track diameter is estimated to be $8 \mathrm{~nm}$ as proposed by Schwen [22,23]. The ratio $\tau$ of the sample surface affected by irradiation can be calculated by the following empirical formula derived by Riedel and Spohr [174]:

$$
\tau=1-\exp \left(-r_{\mathrm{t}}^{2} \pi \tilde{J}\right)
$$

where $r_{\mathrm{t}}$ is the track radius and $\tilde{J}$ is the fluence. For a small surface coverage $(<1 \%)$ track overlap can be ignored and $\tau$ can be approximated by $\tau \approx r_{\mathrm{t}}^{2} \pi \tilde{J}$. The area assumed for irradiated samples is therefore $A_{\mathrm{t}} \approx \tau A_{\text {pad }}$. Furthermore, the current measured on irradiated films $\left(I_{\mathrm{i}}\right)$ is corrected by the current flowing through the unirradiated matrix, by subtracting the current measured through the unirradiated film $I_{\mathrm{u}}$. The track current $I_{\mathrm{t}}$ is approximated to be:

$$
I_{\mathrm{t}}=I_{\mathrm{i}}-I_{\mathrm{u}} \cdot \frac{A_{\mathrm{u}}-A_{\mathrm{t}}}{A_{\mathrm{u}}} .
$$

This expression can be simplified to $I_{\mathrm{t}} \approx I_{\mathrm{i}}-I_{\mathrm{u}}$ if $A_{\mathrm{t}} \ll A_{\mathrm{u}}$. All data of the irradiated samples were corrected in this manner by using the data of the corresponding unirradiated films. Since all unirradiated and irradiated films with a particular $\mathrm{Cu}$ 
concentration were fabricated at the same time, the inhomogeneity of copper concentration was expected to be small. However, the sample thickness may vary by about $5 \%$ to $10 \%$ resulting in an uncertainty of $10 \%$ to $25 \%$ in matrix conductivity (see chapter 5.5. This error is systematic to each measurement comparing two particular contact pads (difference irradiated and unirradiated). Fortunately, by the irradiation the total current increase is in excess of a factor of ten with the exception of the $5 \%$ $\mathrm{Cu}$ sample. The maximum error caused by the matrix-current correction is smaller than $2.5 \%$ for samples with less than $5 \% \mathrm{Cu}$. At low temperature, the residual conductivity, fluctuating strongly between different contact pads, may surpass the effect of irradiation making the correction very inaccurate at temperatures below $50 \mathrm{~K}$.

The fitting of all characteristics was performed by a batch script implemented in python v2.7 [172] using scipy.optimize.curve_fit of scipy 173$]^{3}$. For the parameter $\sigma_{0}$, the initial guess was estimated by calculating the linear slope of the data-point with the smallest bias voltage. The starting value for parameter $\beta$ was calculated by subtracting the linear portion $\sigma_{0} \cdot x$ of the current measured at the largest voltage. The $\gamma$ was not linearly independent of $\beta$ becoming an increasing problem as curvature decreases; the parameter was overdetermined and no stable convergence of the fit was guaranteed. To avoid this problem multiple fits were performed with different fixed $\gamma$ values. At the end, the exponent yielding the smallest error square was chosen together with the accordingly fitted $\sigma_{0}$ and $\beta$ values. This method ensures the finding of the true minimum throughout the whole parameter-space of $\gamma$. Since the range of $\gamma$ is small, this brute force approach was most simple to implement and yielded a precision of 0.002 by performing 250 least square fits, taking a reasonable time of about one second on a typical desktop PC.

The negative and positive branches (bias) were fitted separately to allow for asymmetry of the characteristics. The averaged $\gamma$ was used for both branches. The fit yielded mathematically stable results, the statistical variance of the fitting parameters were smaller than $5 \%$.

\subsubsection{Discussion of temperature dependence of zero-bias conductivity}

\section{Overview of zero-bias conductivity}

In the following plot, figure 5.16, the extrapolated zero-bias conductivities $\sigma_{0}(T)$ are shown for all films. The figure offers a multitude of information in a single overview. First, for different copper concentrations the comparison of the temperature-dependent zero-bias conductivity is given for tracks and matrix. The impurity effect is larger on the matrix than on tracks. The matrix-values of $\sigma_{0}(T)$ increase by three to four orders of magnitude from $0 \%$ to $5 \%$ copper content. The increase correlates with copper concentration with the exception of the $0.1 \%$-sample. All track-ensembles have much higher conductivities than the mere current comparison suggests because

\footnotetext{
${ }^{3}$ http://docs.scipy.org/doc/scipy/reference/generated/scipy.optimize.curve_fit.html
} 
the effective contact area of the tracks is very small and the current density is large. From $0 \%$ to $1 \%$ the values of $\sigma_{0}(T)$ of the tracks increase by a factor of eight, at higher impurity concentration, the values decreases. Secondly, the shape of the temperature dependence of $\sigma_{0}(T)$ is similar for all curves. Finally, the Arrhenius presentation of the data reveals a deviation from a thermally-activated process. At about $200 \mathrm{~K}$ the slope decreases, at this point the pure FP model starts to fail (chap$\operatorname{ter} 5.5$.

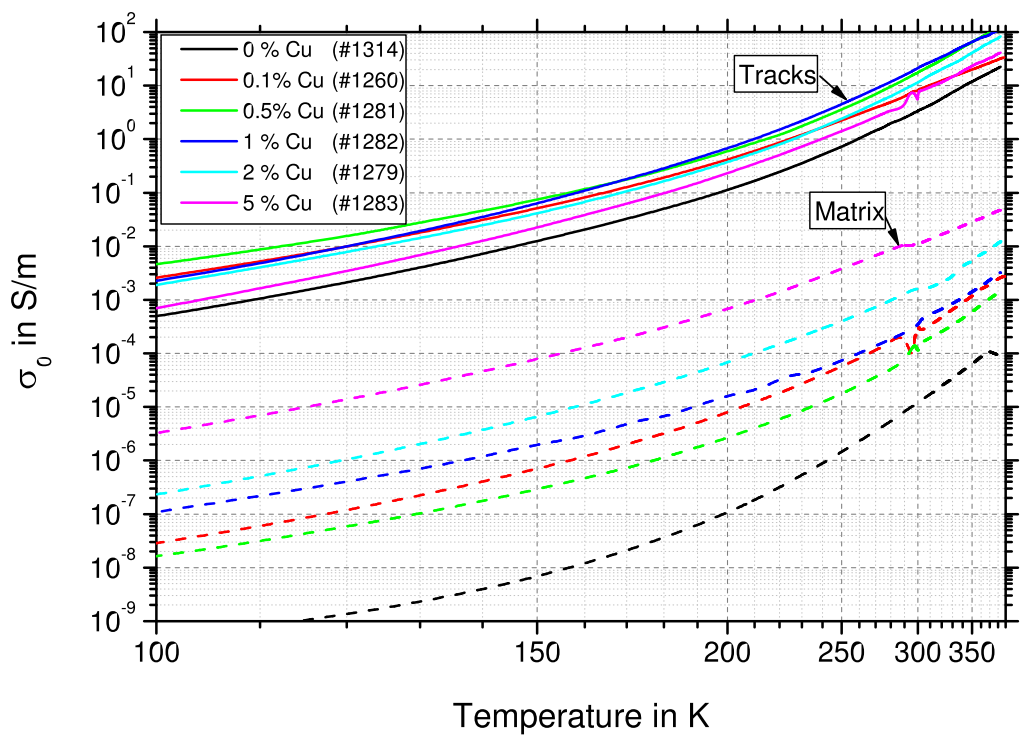

Figure 5.16: The extrapolated zero-bias conductivity $\sigma_{0}(T)$ of irradiated and unirradiated ta$\mathrm{C}: \mathrm{Cu}$ at all available copper concentrations and temperatures. The extrapolation to zero-bias was performed by fitting the field dependence with the stretched exponential model.

\section{Fit model for $\sigma_{0}(T)$}

The deviation from the Arrenius-like behavior towards lower temperatures suggests additional transport mechanisms. In an attempt to describe the transition of the different conduction mechanisms, a sum of three model functions is proposed:

- A thermally activated process $\sigma_{0}^{\mathrm{fp}}$, identified as FP like (chapter 5.5),

- a VRH-term $\sigma_{0}^{\mathrm{vrh}}$ as suggested for tracks by Nix et al. [33] and Gehrke [27],

- and a temperature-independent term $\sigma_{0}^{\mathrm{r}}$ describing residual conductivity.

All three mechanisms in parallel lead to the following formulation of $\sigma_{0}(T)$ :

$$
\sigma_{0}(T)=\sigma_{0}^{\mathrm{fp}}(T)+\sigma_{0}^{\mathrm{vrh}}(T)+\sigma_{\mathrm{r}} .
$$


Applying the theoretical models (equation 2.18 and equation 2.41) leads to the following fit-function:

$$
\sigma_{0}(T)=a_{1} \exp \left(\frac{-q b_{1}}{k_{\mathrm{B}} T}\right)+a_{2} \exp \left(\frac{-b_{2}}{T^{1 / 4}}\right)+c,
$$

where $a_{\mathrm{i}}, b_{\mathrm{i}}$ and $c$ are fit parameter. The parameter $a_{\mathrm{i}}$ represent the contributions of each conduction mechanism while $b_{\mathrm{i}}$ are the temperature dependencies. The residual conductivity is fitted by the constant $c$. The fits were performed using the fitting routine of Origin 7.0 implementing the Levenberg-Marquardt-Algorithm. $b_{1}$ was fitted at high temperatures, where thermally activated processes were dominant and $b_{2}$ was fitted below $200 \mathrm{~K}$ in the regime of VRH. Finally, the $a$ parameters were readjusted fitting the complete range keeping the $b$-parameter constant. With this method, stable and reproducible fit results could be obtained for the temperature dependencies. The proportional factors $a_{\mathrm{i}}$ show a strong correlation to the respective $b_{\mathrm{i}}$ variable. Therefore, a small error in $b_{\mathrm{b}}$ will produce a large error in $a_{\mathrm{i}}$.

An example of the resulting fits is shown in figure 5.17 for ion tracks in pure ta-C. The temperature scale is transformed to $\mathrm{K}^{-1 / 4}$ in order present the VRH temperature dependence as a straight line. The plot nicely shows each mechanism dominating a certain temperature regime, even the residual conductivity is well fitted with this approach. Therefore, the zero-bias conductivity of ta-C ion-tracks can be described by three conduction mechanisms in the range of $20 \mathrm{~K}$ to $400 \mathrm{~K}$.

In the following, all $\sigma_{0}(T)$ values are shown together with their fit. In figure 5.18 the results for the unirradiated ta-C:Cu films are shown. In general, the model describes the temperature dependence of $\sigma_{0}(T)$ well. Although a close look shows some deviations. The conductivity of pure ta- $\mathrm{C}$ is not described precisely around $50 \mathrm{~K}$ and below, showing some limitations of the modeling of the residual conductivity. The data shows some straggling caused by fitting the $I$ - $V$-characteristics in order to extract $\sigma_{0}(T)$ that is most visible around $300 \mathrm{~K}$ where low- and high-temperature setup data have been merged. One data point of the $0.1 \%$ sample, an outlier, was ignored during the fit. As already seen in figure 5.16, the effect of the copper impurities causes an increase in conductivity of up to three orders of magnitude. The increase is largest from pure to slightly doped ta- $\mathrm{C}$ and at lower temperatures. The transition from VRH to FP is apparently shifted to higher temperatures with increasing copper concentration. The residual conductivity is visible at about $20 \mathrm{~K}$ for some samples; for pure ta-C and the $0.5 \%$ doped films it is off scale. The residual conductivity does not correlate with $\mathrm{Cu}$ concentration. Except for $0.1 \%$ $\mathrm{Cu}$, the conductivity correlates with $\mathrm{Cu}$ concentration. The zero-bias conductivity of this film exhibits slightly larger values than the film with $0.5 \% \mathrm{Cu}$ content most likely caused by inhomogeneities of the $0.1 \% \mathrm{Cu}$ sample.

The overall temperature dependence of $\sigma_{0}(T)$ is similar to the dependence of the current shown at the beginning of the chapter. However, at low temperatures, where the curvature of the $I$ - $V$-curves is largest, the values of $\sigma_{0}(T)$ decrease faster than 


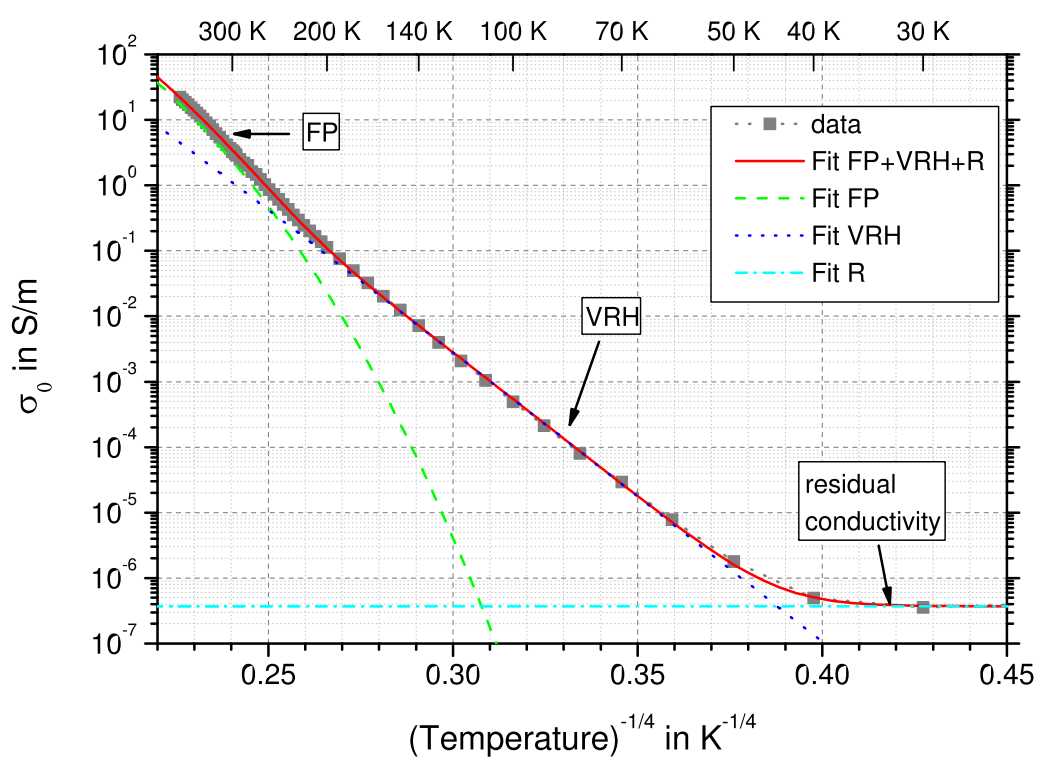

Figure 5.17: $\ln \left[\sigma_{0}(T)\right]$-plot of ion tracks in pure ta-C versus $T^{-1 / 4}$ (VRH behavior becomes linear. In addition, the fit according to equation 5.11 is plotted, including the individual terms representing each a conduction model. The thermal activation (FP) is dominant at high temperatures. Towards lower temperatures VRH takes over and below $40 \mathrm{~K}$ the temperature independent residual conductivity is observed. All mechanisms together describe the data well.

the current values. Therefore, the range of the zero-bias conductivity is even larger reaching more than eight orders of magnitude over the temperature range.

The results for $\sigma_{0}(T)$ of the ion-tracks are shown in figure 5.19. As already seen in the first overview of the zero-bias data, the effect of the copper is much smaller than in case of the matrix. The copper concentration yielding the highest track conductivity (factor eight) is about $0.5 \%$ to $1 \%$. Again, curves of $\sigma_{0}(T)$ look similar to the temperature-dependent current plots. At low temperatures, some deviation of the model and the data is observed, especially in case of $0.5 \% \mathrm{Cu}$. Otherwise, the data is fitted well by the model. 


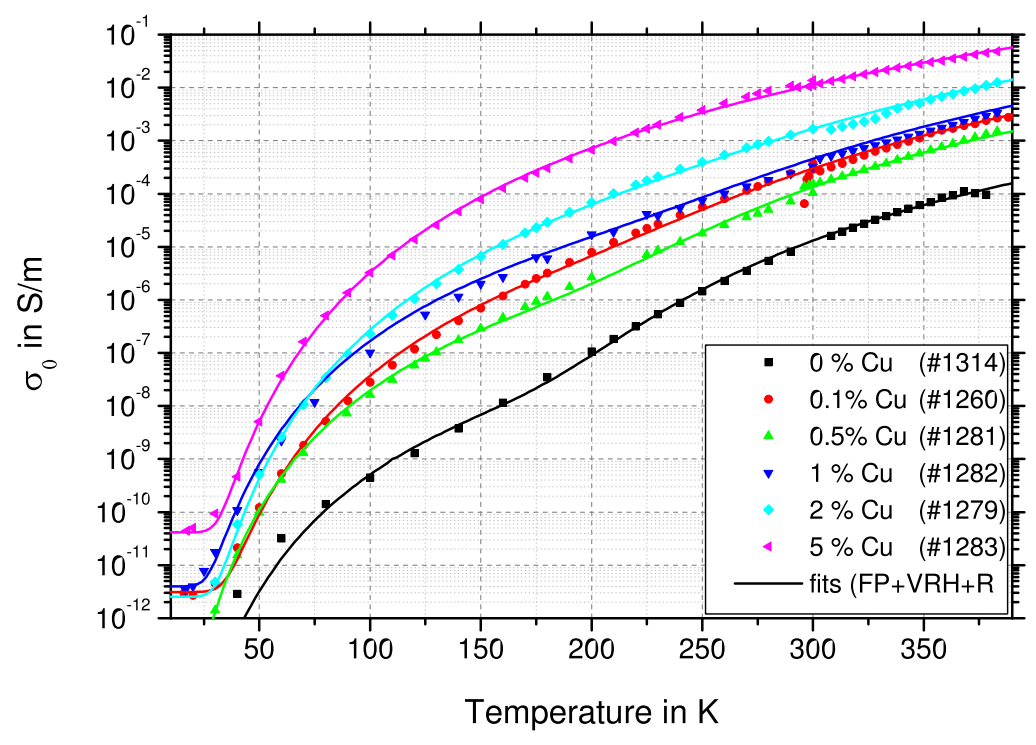

Figure 5.18: The extrapolated zero-bias conductivity $\sigma_{0}(T)$ of all unirradiated samples. The solid lines are fits with the sum of the Frenkel-Poole, variable-range-hopping, and residual-conductivity contributions.

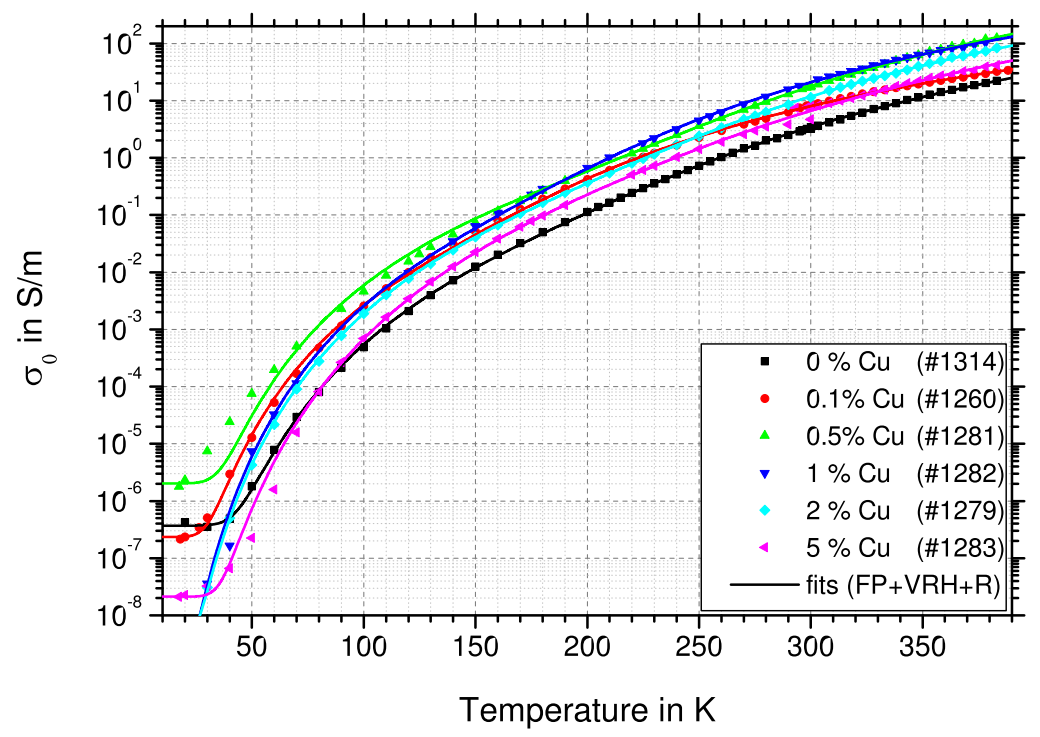

Figure 5.19: The extrapolated zero-bias conductivity $\sigma_{0}(T)$ of all irradiated samples. The solid lines are fits with the sum of the Frenkel-Poole, variable-range-hopping, and residual-conductivity contributions. 


\section{Presentation of fit parameter}

Two tables, table 5.2 and table 5.3. summarize the fit parameters used to simulate $\sigma_{0}(T)$ by equation 5.11. The $a_{\mathrm{i}}$ parameters are proportional factors multiplied with the exponential function. Their values correlate strongly with $b_{\mathrm{i}}$ and should be carefully compared. The $b_{\text {i }}$ parameter, portraying the temperature dependence, yield more information of the physical processes in the film. $b_{1}$ is the activation energy for the thermally activated high temperature conductance process. The $b_{2}$ parameter is equivalent to $B$ as defined in the VRH mechanism (equation 2.44). The transition temperature $T_{\text {trans }}$ is the temperature, where both FP and VRH conduction mechanisms have equal contributions. At higher temperatures, the thermal activation dominates $\left(b_{1}\right)$, below, the VRH model $\left(b_{2}\right)$. The parameters are given with their respective errors estimated by the fit-algorithm.

\begin{tabular}{|c||c|c|c|c|c|c|}
\hline $\begin{array}{c}\mathrm{Cu} \\
{[\%]}\end{array}$ & $\begin{array}{c}a_{1} \\
{\left[\mathrm{~S} \mathrm{~m}^{-1}\right]}\end{array}$ & $\begin{array}{c}b_{1} \\
{[\mathrm{eV}]}\end{array}$ & $\begin{array}{c}a_{2} \\
{\left[\mathrm{~S} \mathrm{~m}^{-1}\right]}\end{array}$ & $\begin{array}{c}b_{2} \\
{\left[\mathrm{~K}^{25}\right]}\end{array}$ & $\begin{array}{c}c \\
{\left[\mathrm{~S} \mathrm{~m}^{-1}\right]}\end{array}$ & $\begin{array}{c}T_{\text {trans }} \\
{[\mathrm{K}]}\end{array}$ \\
\hline 0 & $0.679(122)$ & $0.281(5)$ & $1.44(119) \times 10^{4}$ & $90.7(27)$ & $5.0(5) \times 10^{-14}$ & $192(2)$ \\
0.1 & $11.5(17)$ & $0.280(10)$ & $1.82(11) \times 10^{8}$ & $107(2)$ & $3.1(3) \times 10^{-12}$ & $249(7)$ \\
0.5 & $5.77(18)$ & $0.278(7)$ & $9.98(35) \times 10^{5}$ & $92.5(8)$ & $7.8(8) \times 10^{-14}$ & $220(5)$ \\
1 & $19.2(10)$ & $0.284(17)$ & $1.86(10) \times 10^{7}$ & $95.0(21)$ & $4.0(4) \times 10^{-12}$ & $261(8)$ \\
2 & $40.1(7)$ & $0.284(6)$ & $1.15(21) \times 10^{10}$ & $114(1)$ & $2.5(3) \times 10^{-12}$ & $308(12)$ \\
5 & $5.38(7218)$ & $0.258(42)$ & $1.50(53) \times 10^{11}$ & $114(1)$ & $4.1(4) \times 10^{-11}$ & - \\
\hline
\end{tabular}

Table 5.2: The fit parameters of the zero-bias fit of $\sigma_{0}(T)$ (equation 5.11) for unirradiated ta-C:Cu. In addition, the transition temperature, where $\sigma_{0}^{\mathrm{fp}}=\sigma_{0}^{\mathrm{vrh}}$ is displayed. Below this temperature, the system is dominated by VRH, above by FP. In addition to all parameters, errors were calculated by the fit algorithm.

\begin{tabular}{|c||c|c|c|c|c|c|}
\hline $\begin{array}{c}\mathrm{Cu} \\
{[\%]}\end{array}$ & $\begin{array}{c}a_{1} \\
{\left[\mathrm{kS} \mathrm{m}^{-1}\right]}\end{array}$ & $\begin{array}{c}b_{1} \\
{[\mathrm{eV}]}\end{array}$ & $\begin{array}{c}a_{2} \\
{\left[\mathrm{~S} \mathrm{~m}^{-1}\right]}\end{array}$ & $\begin{array}{c}b_{2} \\
{\left[\mathrm{~K}^{.25}\right]}\end{array}$ & $\begin{array}{c}c \\
{\left[\mathrm{~S} \mathrm{~m}^{-1}\right]}\end{array}$ & $\begin{array}{c}T_{\text {trans }} \\
{[\mathrm{K}]}\end{array}$ \\
\hline 0 & $24.8(26)$ & $0.239(4)$ & $3.20(48) \times 10^{12}$ & $108(1)$ & $3.7(4) \times 10^{-7}$ & $249(17)$ \\
0.1 & $4.63(50)$ & $0.171(4)$ & $1.60(45) \times 10^{11}$ & $93.1(9)$ & $2.3(3) \times 10^{-7}$ & $194(29)$ \\
0.5 & $254(154)$ & $0.255(17)$ & $5.73(387) \times 10^{11}$ & $94.6(23)$ & $2.0(2) \times 10^{-6}$ & $268(12)$ \\
1 & $62.8(98)$ & $0.214(10)$ & $1.28(35) \times 10^{13}$ & $107(1)$ & $1.2(1) \times 10^{-9}$ & $217(19)$ \\
2 & $126(12)$ & $0.250(3)$ & $1.01(8) \times 10^{13}$ & $107(1)$ & $3.3(3) \times 10^{-9}$ & $254(27)$ \\
5 & $66.3(87)$ & $0.260(15)$ & $2.49(117) \times 10^{14}$ & $121(2)$ & $2.1(2) \times 10^{-8}$ & $326(32)$ \\
\hline
\end{tabular}

Table 5.3: The fit parameters of the zero-bias fit of $\sigma_{0}$ (equation 5.11) for irradiated ta-C:Cu. In addition, the transition temperature, where $\sigma_{0}^{\mathrm{fp}}=\sigma_{0}^{\mathrm{vrh}}$ is displayed. Below this temperature, the system is dominated by VRH, above by FP. In addition to all parameters, errors were calculated by the fit algorithm.

First, in table 5.2 the data of the unirradiated ta-C is shown in dependence of the copper concentration. The activation energy $\left(b_{1}\right)$ of the high temperature thermally activated transport is almost not affected by the copper concentration being stable 
at about $0.28 \mathrm{eV}$. This value is in good agreement with findings of other experiments 134 137, 175. The FP fit (chapter 5.5) resulted in a slightly lower value of $0.25 \mathrm{eV}$ caused by the different fitting procedures.

The low temperature fitting variable of the VRH part $\left(b_{2}\right)$ seems to increase from $90 \mathrm{~K}^{0.25}$ to $114 \mathrm{~K}^{0.25}$ being equivalent to a reduction of $\alpha^{-1}$ by $25 \%$ or reduction of $N\left(E_{\mathrm{F}}\right)$ by a factor of about 2.5 according to Mott's model (equation 2.44). Assuming $\alpha^{-1}$ to be of the order of $0.1 \mathrm{~nm}$ to $1 \mathrm{~nm}$ (order of Bohr radius) suggests $N\left(E_{\mathrm{F}}\right)$ to be in the range of $1 \times 10^{18} \mathrm{~cm}^{-3} \mathrm{eV}^{-1}$ to $1 \times 10^{21} \mathrm{~cm}^{-3} \mathrm{eV}^{-1}$ for $B=90 \mathrm{~K}^{1 / 4}$. A value of $B=120 \mathrm{~K}^{1 / 4}$ would result in a DOS of $3 \times 10^{17} \mathrm{~cm}^{-3} \mathrm{eV}^{-1}$ to $3 \times 10^{20} \mathrm{~cm}^{-3} \mathrm{eV}^{-1}$. These values correspond to the results of Maiken and Taborek using a similar approach to the Mott formula to fit the data of a-C [176]. Electron Spin Resonance (ESR) measurements suggest values for $N\left(E_{\mathrm{F}}\right)$ in the order of $7 \times 10^{19} \mathrm{~cm}^{-3} \mathrm{eV}^{-1}$ to $3 \times 10^{20} \mathrm{~cm}^{-3} \mathrm{eV}^{-1}$ [148]. However, the Mott equation is not very good in calculating the DOS because of the extensive simplifications made to the hopping range and DOS in general. The results merely suggest physical reasonable results for $B$.

The transition temperature, dividing the data in VRH and FP dominated regimes, increases with $\mathrm{Cu}$ concentration. For pure ta-C it is $192 \mathrm{~K}$, which would again explain the failure of a solely thermally activated model below $200 \mathrm{~K}$ as shown in chapter 5.5. At $2 \% \mathrm{Cu}$, the transition takes place above room temperature at $308 \mathrm{~K}$ and finally, the $5 \% \mathrm{Cu}$ sample shows no transition temperature at all. The VRH is dominant at the whole temperature range measured. At some point a transition would be expected, however, measurements at higher temperatures would have been required to fit it. As a side effect, the value of $b_{1}$ obtained for $5 \% \mathrm{Cu}$ is less precise than the other values because no pure thermally activated regime has been available for fitting. The slightly lower activation energy of $0.258(42) \mathrm{eV}$ cannot be interpreted as a dropping of activation energy for high copper concentrations. However, at increasing impurity concentrations a smaller activation energy is plausible, assuming increased nearest neighbor hopping and reduced effective barrier-heights by higher DOS.

The second table 5.3 shows the data of the tracks in ta-C. The values for the thermal activation $b_{1}$ are smaller and show more variation. The fluctuation of the data does not allow a conclusion of a dependence on copper concentration. The VRH temperature dependence is comparable to the data of the matrix, the values are in the same range. Again, $b_{2}$ seems to increase slightly meaning either a decrease of $N\left(E_{\mathrm{F}}\right)$ or $\alpha^{-1}$. The transition temperature of tracks in pure ta-C is significantly higher reaching almost room temperature. Nix and Gehrke were able to fit tracks in ta-C with only VRH for temperatures below $300 \mathrm{~K}$ calculating values for $B$ between $82 \mathrm{~K}^{0.25}$ and $93 \mathrm{~K}^{0.25} 27,32,33$. The tracks in ta-C:Cu show only a small statistical relevant difference in their transition temperatures. In fact, the values are affected by large errors and fluctuation. In general, a higher transition temperature means a larger contribution of the VRH.

Considering the data presented in both tables, the most interesting aspect is the quasi independence of the temperature dependencies on copper concentration and 
irradiation. The main effect of irradiation and copper is the increase of the proportional parameters $a_{\mathrm{i}}$ - especially the prefactor of VRH. In tracks and copper-rich samples, VRH is more dominant because more hopping sides are created and tunneling transport becomes more important. In addition, the thermal activation energy is also hardly affected by irradiation and copper impurities. The transport mechanism and the energy levels seem to remain untouched, only the total number of charge carriers increases. In the scope of this model, the effect of copper and irradiation are very similar, in both cases hopping becomes more dominant. The VRH regime extends to higher temperatures increasing the transition temperature.

\section{Deviations of sample with $0.1 \% \mathrm{Cu}$}

The sample with about $0.1 \% \mathrm{Cu}$ shows some non-consistence results for $\sigma_{0}(T)$ compared to the other $\mathrm{Cu}$ concentrations. The matrix conductivity is higher than compared to $0.5 \% \mathrm{Cu}$, otherwise $\mathrm{Cu}$ concentration scales with conductivity. The tracks have a comparatively high conductivity at low temperatures and at high temperatures the values drop to the conductivity of tracks in pure ta-C. The explanation could be either an outlier of the sample per se. It has to be kept in mind, that unlike the other samples, this film has not constantly been doped with copper but rather contaminated by accident during the sputtering process prior to film deposition. Although Copper has the tendency to accumulate at the surface [153, 158], it is most likely distributed though the whole film. The concentration gradient of this film profile is most likely not the same as in other purposely doped samples. Furthermore, the concentration of the copper is not homogeneously spread over the film area as in case of intentionally doped samples. Finally, the sputtered copper was not mass-selected and probably oxidized partially before being incorporated into the ta-C.

\section{Temperature-dependent activation energy}

As a last thought on $\sigma_{0}(T)$, the transition of the different conduction mechanisms should be visualized by plotting the temperature-dependent thermal activation $E_{\mathrm{A}}$. This has been accomplished by deriving $\ln \sigma_{0}(T)$ by $\frac{1}{T}$. If a single thermal activation process is dominant, a constant derivative is expected. Numerically, the data is derived by the following formula:

$$
E_{\mathrm{A}}(T)=\frac{\mathrm{d}}{\mathrm{d} \frac{1}{T}} k_{\mathrm{B}} \ln \left(\sigma_{0}(T)\right) \approx k_{\mathrm{B}} \ln \left(\frac{\sigma_{1}}{\sigma_{2}}\right) \frac{T_{1} T_{2}}{T_{2}-T_{1}},
$$

where $T_{1}$ and $T_{2}$ are temperatures of two consecutive measurements of $\sigma T$ ( $\sigma_{1}$ and $\left.\sigma_{2}\right)$. The fit function equation 5.11 is analytically derived and plotted in comparison to the data. In figure 5.20 the results for the ta- $\mathrm{C}$ matrix are shown for all copper concentrations. The fits show the transitions of the conduction models. At about 
$30 \mathrm{~K}$, the residual conductivity is surpassed by the $\mathrm{VRH}$, where the slope starts rising to about $0.05 \mathrm{eV}$. Up to $150 \mathrm{~K} E_{\mathrm{A}}$ increases linearly until the thermally activated FP mechanism becomes more dominant. At this point, the activation energy increases rapidly approaching the fitted value of $b_{1}$. This transition is quite sharp in case of pure ta-C . As the copper concentration increases, the transition smears out over a larger temperature range. In the extreme case of $5 \% \mathrm{Cu}$, no transition is observed in the measured temperature domain. The transition from linear to constant slope is the regime, where $\sigma_{0}$ cannot be well described by a single mechanism. This transition takes place slightly below room temperature. Therefore, only the combination of both high- and low-temperature measurements allows the correct recognition of the processes around $300 \mathrm{~K}$.

The data points are well described by the fits at low temperature, at room temperatures, the data becomes more noisy. The reason is on one hand the increasing error in $\frac{1}{T}$ and on the other hand the smaller step width in $T$ increasing the error caused by $\ln \frac{\sigma_{1}}{\sigma_{2}}$. Nonetheless, the tendency of the data is reproduced by the fit.

In case of the tracks, displayed in figure 5.21. many similarities to the matrix are visible. Again, at low temperatures the transition takes place from residual to $\mathrm{VRH}$ conduction at $30 \mathrm{~K}$. The linearly-increasing activation energy toward $150 \mathrm{~K}$ has the same slope as in case of the unirradiated films (similar $b_{2}$ values). At high temperatures, the transition to the thermally activated conduction is less pronounced than in case of the matrix. No significant effect of copper is visible (except $0.1 \%$ ) as the effect of copper on track conductivity is smaller. The data fits the simulation well at low temperatures, beyond $300 \mathrm{~K}$ the noise of the data increases because of increasing margins of errors of the numerically calculated derivatives.

The plots of the temperature-dependent activation energies visualize the reason for the variation of literature values of thermal activation energy of amorphouscarbon. Often, only small temperature domains are used to extract the slope. Around room temperature, a temperature regime most conveniently used for measurements, the activation is not constant. Furthermore, the temperature dependence of the activation energy visualizes the change of the importance of tunneling (hopping). At low temperatures, tunneling is more likely and the resulting activation energy is small. Towards higher temperature, the thermally activated transport gains importance leading to a total dominance at high temperatures, where a constant activation energy is seen. 


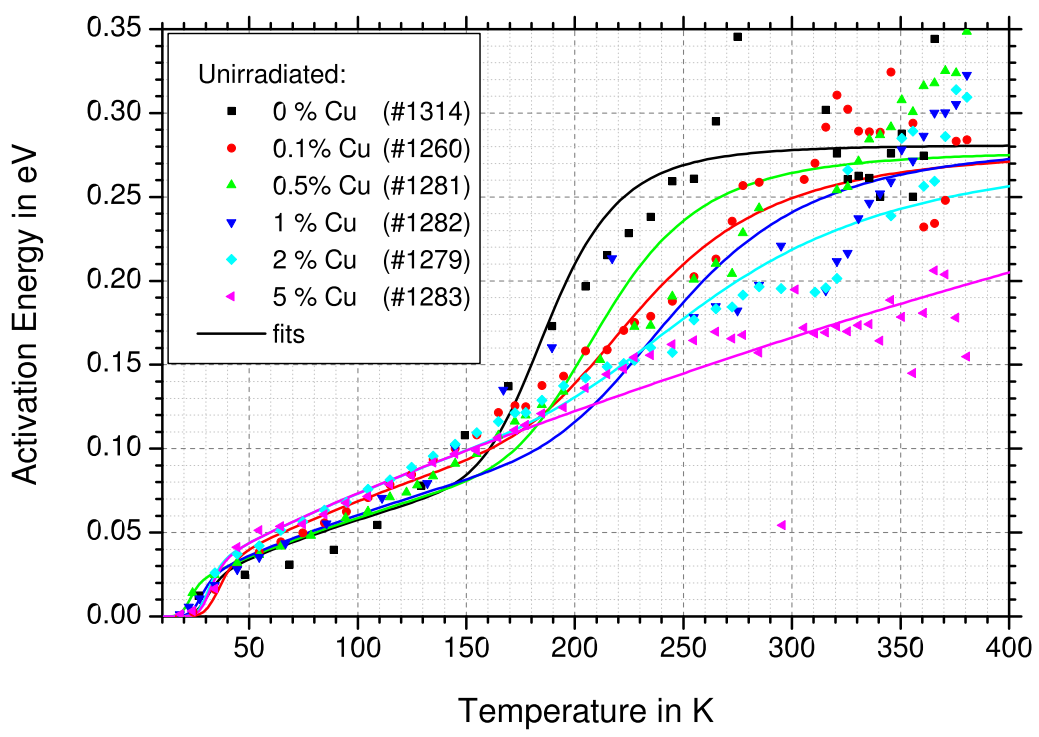

Figure 5.20: Temperature-dependent activation energy (derivative of $\ln \left[\sigma_{0}(T)\right]$ to $1 / T$ ) for unirradiated ta-C: $\mathrm{Cu}$. The residual conductivity has an activation of zero, followed by a monotonously increasing activation energy in the VRH regime. At the transition temperature between the VRH and the FP regime, the activation energy shows a steep increase towards the value of the barrierheight. At high temperatures, the activation becomes temperature independent. The addition of copper increases the transition temperature.

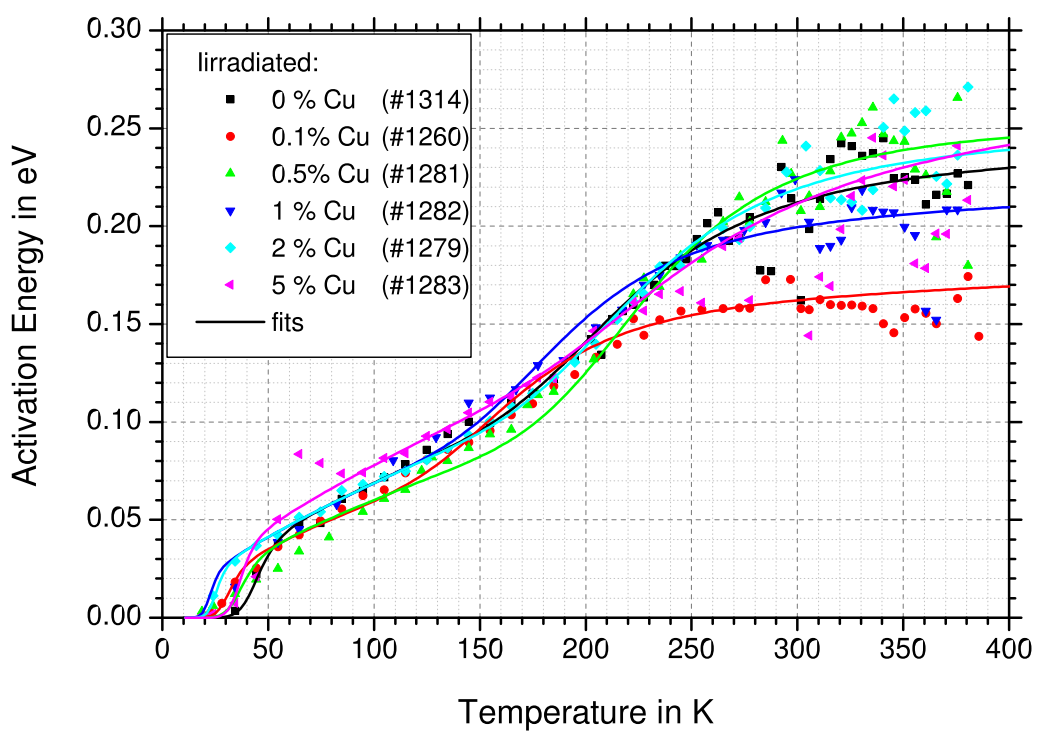

Figure 5.21: Temperature-dependent activation energy (derivative of $\ln \left[\sigma_{0}(T)\right]$ to $1 / T$ ) for irradiated ta-C:Cu. Again, the residual conductivity has an activation energy of zero, followed by a monotonously increasing activation energy in the VRH regime. At the transition energy the thermal activated FP causes an increase towards the value of the barrier-height, which is not as steep as in case of unirradiated ta-C. At high temperatures, the activation becomes temperature-independent. 


\subsubsection{Discussion of curvature of the $I-V$-characteristics}

After discussing and modeling the zero-bias conductivity, the curvature of the $I-V$ characteristics will be investigated. The fit has two parameters describing the curvature: $\beta$ and $\gamma$ (equation 5.7). The high temperature thermally activated mechanism has been successfully fitted with an extended FP model (chapter 5.5). The fitted curvature of the $I$ - $V$-data shall be compared to the expected curvature of the Frenkel-Poole model:

$$
\sigma_{0}^{\mathrm{fp}} \exp \left(\frac{1}{k_{\mathrm{B}} T} \sqrt{\frac{q^{3} F}{\pi \epsilon \epsilon_{0}}}\right)=\sigma_{0}^{\mathrm{fit}} \exp (\beta F)^{\gamma} .
$$

This equation can be solved for $\beta$ assuming $\gamma=0.5$ and $\sigma_{0}^{\mathrm{fp}}=\sigma_{0}^{\mathrm{fit}}$, where $\sigma_{0}^{\mathrm{fit}}$ is the fitted value obtained in the previous section:

$$
\beta=\frac{1}{T^{2}} \frac{q^{3}}{\pi \epsilon \epsilon_{0} k_{\mathrm{B}}^{2}}=\frac{1}{T^{2}} \frac{0.776\left[\mathrm{~V}^{-1} \mathrm{~m}\right]}{\epsilon} .
$$

In case of a fixed $\epsilon, \beta T^{2}$ should be constant. In figure 5.22 $\beta T^{2}$ is plotted for matrix and tracks at all copper concentrations. The $\beta$ values used were fitted with a fixed exponent $\gamma=0.5$. On the right side of the plot the values of $\epsilon$ are displayed according to equation 5.14. The plot shows no constant regime of $\epsilon$ in any film, in particular the $\epsilon$ of the unirradiated samples vary strongly, the irradiated samples (tracks) show smaller variations. The $\epsilon$ values of the pure ta-C matrix are in the order of 15 to 20 in the $150 \mathrm{~K}$ to $300 \mathrm{~K}$ region. These values are about three to four times larger than expected from optical measurements [37]. However, this result is not unusual for a FP fit as discussed in chapter 2.5.1. Especially the argument by Simmons should be considered, explaining four times larger values of $\epsilon$. In light of the small activation energy of about $0.3 \mathrm{eV}$, shown for $\sigma_{0}(T)$, that is most likely not the distance from the Fermi energy to the conduction band; the argument of Simmons seems plausible for ta-C. If copper is added to the matrix, the apparent $\epsilon$ increases further. The tracks show apparent epsilon values, which are a factor 100 above the expectations. Here, the increasing influence of hopping is most likely responsible for the results as demonstrated by Hall and Hill. Therefore, in the sense of this work, Frenkel-Poole more generally refers to thermally activated transport, including hopping.

As it has been already noted by Mott [125], the prefactors of conduction models are hard to determine by theoretical approaches, since many processes are in competition to each other and extended knowledge about properties, such as precise DOS or phonon frequency would be required. For this reason, VRH and FP are usually formulated as proportionalities. $\sigma_{0}$ fitted to the characteristics is the sum of all prefactors of the conduction mechanisms. In turn, $\beta$ is the total curvature parameter. The assumption $\sigma_{0}^{\mathrm{fp}}=\sigma_{0}^{\mathrm{fit}}$ is not fulfilled if another mechanism besides FP or a modification of FP has a significant contribution. Equation 5.14 is therefore not 
a reliable method to derive $\epsilon$. Unfortunately, the sum of $x a_{\mathrm{i}} \exp \left(b_{\mathrm{i}} x\right)$ cannot be written as $x a \exp (b x)$. It can only be approximated. In the following equation this approximation is illustrated for the sum of a FP resistor and an ohmic resistor $\left(\sigma_{\mathrm{x}}\right)$ :

$$
\sigma_{0}^{\mathrm{fp}} \exp \left(\frac{1}{k_{\mathrm{B}} T} \sqrt{\frac{q^{3} E}{\pi \epsilon \epsilon_{0}}}\right)+\sigma_{\mathrm{x}}=\sigma_{0}^{\mathrm{fp}} \exp \left(\sqrt{\beta_{\mathrm{fp}} E}\right)+\sigma_{\mathrm{x}} \approx \sigma_{0}^{\mathrm{fit}} \exp \left(\sqrt{\beta_{\mathrm{fit}} E}\right) .
$$

Now $\beta_{\text {fit }}$ has to be smaller than $\beta_{\text {fp }}$. A first order approximation is $\beta_{\text {fit }} \approx \frac{\sigma_{0}^{\mathrm{fp}} \beta_{\mathrm{fp}}}{\sigma_{0}^{\mathrm{fp}}+\sigma_{\mathrm{x}}}$ assuming $\sigma_{0}^{\mathrm{fit}} \approx \sigma_{0}^{\mathrm{fp}}+\sigma_{\mathrm{x}}$. In the case of other mechanisms present in parallel (e.g. hopping), the fitted $\beta$ yields a smaller curvature (larger $\epsilon$ ) than expected by the original FP model. This result is in accordance with theoretical expansions of the FP model (chapter 2.5.1). Therefore, the more physical explanation of apparently high $\epsilon$ values fitting the FP are deviations form the pure FP mechanism. Toward low temperatures, the parallel VRH becomes more dominant reducing the measured $\beta$. With increasing temperature and copper concentration FP conduction becomes less pronounced as more ohmic behavior is observed as portrayed in figure 5.22, one possible explanation could be barrier exhaustion. Such exhaustion (saturation) is suggested for carbon-nano-tubes described by Perello et al. [177, 178]. Another factor is the increased importance of thermally activated hopping (over-barrier-hopping) at higher temperature.

The copper impurities and the tracks increase the defect density and decrease the localization length enhancing the thermally assisted hopping (over-barrier). In addition, at higher temperatures, the decreased curvature could be the result of barrier saturation of states as suggested by Perello et al. [177, 178. At low temperatures, the VRH contribution is significantly enhanced by the addition of copper and tracks decreasing the influence of FP in turn reducing the curvature.

The jumps at $300 \mathrm{~K}$ in figure 5.22 are caused by the different contacts in the lowand high-temperature setup. Small kinks are already present in the fitting of $\sigma_{0}(T)$ which alternately causes jumps in $\beta$. These kinks are not caused by the physical properties of the samples but are a artifact of the fitting procedure.

The non-linear behavior of the $I-V$-curves at low temperatures, where $\mathrm{VRH}$ is dominant, can be described analytically as well. Based on peculation theory, a model has been created by Pollak and Riess [132]. The field dependence is proportional to $\exp F$ as seen in equation 2.44. The only material dependent variable is the hopping range $R$. Figure 5.23 shows the exponent $\beta$, obtained from fitting the characteristics using a fixed exponent $\gamma=1$. In the plot the product $\beta T$ is plotted against temperature, which is directly proportional to the hopping range $R$ :

$$
\beta=\frac{q \tilde{\gamma} R}{k_{\mathrm{B}} T} \Rightarrow R=\beta T \frac{k_{\mathrm{B}}}{q \tilde{\gamma}},
$$

where $\tilde{\gamma}=0.17$ is a numerical factor of the model by Pollak and Riess. The data of the tracks in pure ta-C show the highest curvature and $R$ values. Between $50 \mathrm{~K}$ 


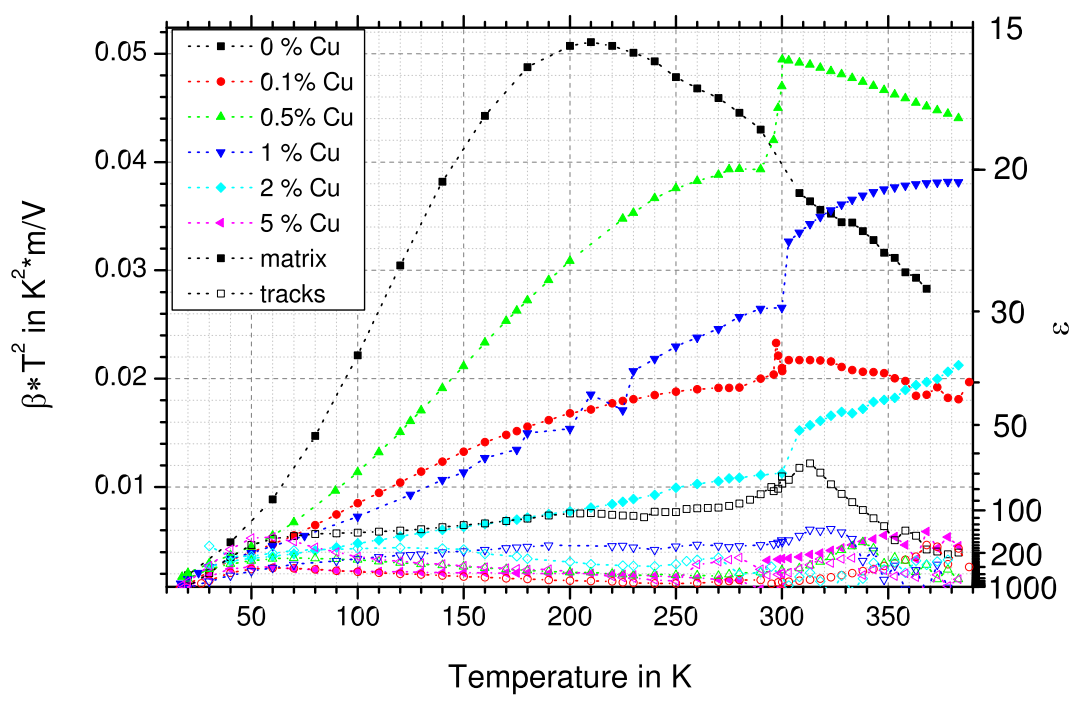

Figure 5.22: Fitting results of the curvature $\beta T^{2}$ for fixed $\gamma=0.5$ of ta-C:Cu matrix and tracks. In case of FP, constant curvatures $(\epsilon)$ would be expected. Towards low temperatures the VRH dominates, the curvature decreases. Copper and tracks reduce the curvature as well.

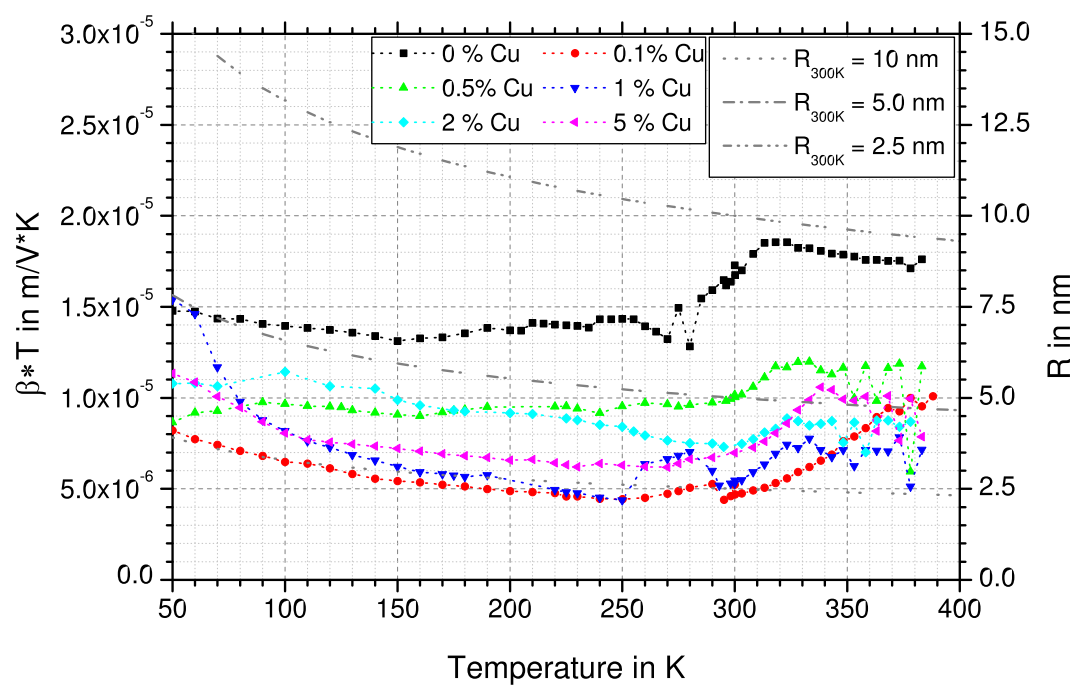

Figure 5.23: The curvature $\beta T$ for tracks in ta-C:Cu. The corresponding hopping ranges, according to Pollak and Riess moderate-field correction are presented. The addition of copper reduces the hopping ranges from about $7 \mathrm{~nm}$ to $2.5 \mathrm{~nm}$. These results support the plausibility of the moderatefield correction. 
to $270 \mathrm{~K}$ the fitted $R$ is almost constant at $7.5 \mathrm{~nm}$ slightly increasing towards low temperatures. Above about $300 \mathrm{~K}$ slightly higher values are found. The same general temperature behavior is seen for the samples with copper impurities but the $R$ values are generally smaller, indicating a reduction of the hopping distance with $\mathrm{Cu}$ concentration. The $R$ values should probably not be interpreted as width of the tunneling barrier, since that would give physically unreasonably-high values, but rather as an average distance between hopping sites. The idea would be the combination of a tunnel barrier followed by a free path making up each hop. For all curves the calculated values for $R$ cannot be interpreded in terms of the moderate field correction at both extremes because of the dominance of the residual conductivity (low temperature) and the thermally activated transport (high temperature). Nix fitted a comparable hopping range of $4 \mathrm{~nm}$ to $6 \mathrm{~nm}$ for ion tracks in ta-C with a slightly different method 32 .

The hopping ranges in unirradiated ta-C matrixes would be well beyond $15 \mathrm{~nm}$. The model by Pollak and Riess does not seem to work well for the ta-C matrix. One first possible explanation could be the perculation approach chosen by Pollak and Riess only looking at small forward angles for possible conduction channels. In the low dimensional tracks, backwards hopping is unlikely because of the confined dimensionality. However, in two dimensional films this confinement is not present, a larger angle of hopping paths is possible. Pollak and Riess admit this weakness of their model.

The hopping range is slightly temperature-dependent $\left(R \propto \frac{1}{T^{1 / 4}}\right)$. Therefore, a small increase of the hopping range is expected towards low temperatures. The grey lines in figure 5.23 indicate the expected progression of $R(T)$ for three different hopping ranges $\left(R_{300 \mathrm{~K}}\right)$. The precision of the data is not well enough to clearly show this relation, in addition, the summation of different parallel conduction mechanisms obstruct the observation of trends. The behavior at low temperatures hints the slight increase expected by the model, although at $50 \mathrm{~K}$ the residual conductivity could be already present at high fields.

The exponent $\gamma$ has been kept constant to fit either the FP emission $(\gamma=0.5)$ or the moderate-field correction for $\operatorname{VRH}(\gamma=1)$. In the following, the results of $\gamma$, treated as free parameter, are presented. These fits are equivalent to the calculations used to determine $\sigma_{0}(T)$ in the first place. In case of the ta- $\mathrm{C}$ : $\mathrm{Cu}$ matrix for concentrations up to $2 \%, \gamma$ was found close to 0.5 . Except for temperatures beyond $330 \mathrm{~K}$ where for some copper concentrations $\gamma$ values up to 0.7 were obtained. The $5 \% \mathrm{Cu}$ sample was best fitted with $\gamma=1$ above $120 \mathrm{~K}$, below this temperature the exponent drops to 0.5 . The tracks behave very similar to the film with $5 \% \mathrm{Cu}$, i.e. at temperatures beyond $120 \mathrm{~K}, \gamma$ is one, at low temperatures the values drop to 0.5 .

The values of $\gamma$ should not be interpreted too intensively because of a high linear dependency with $\beta$ while fitting causing a correlation between $\beta$ and $\gamma$ which makes the individual values uncertain. To obtain good values for $\gamma$, higher bias voltages would be required, especially with increasing copper concentrations and if tracks are 
considered. Nonetheless, the obtained $\gamma$ values hint towards a better description of the track data with the moderate field correction model whereas the matrix data are better described by the $\sqrt{F}$ field dependence. At low temperatures, the residual conductivity dominates the transport which seems to prefer an exponent of 0.5 independent of copper concentration and for both tracks or matrix.

\subsection{Summary of the $I-V$ - $T$-model}

The approach of using the stretched exponential function (equation 5.7) for the field dependence was a successful method to obtain the zero-voltage conductivity which was then studied as a function of temperature and doping. The field dependence was subsequently studied separately. The following conclusions can be drawn from the data and the analysis:

- The temperature dependence of all $\sigma_{0}(T)$ can be explained by three mechanisms: residual conductivity at very low temperatures, Mott variable-rangehopping in the low temperature regime, and a thermally activated FrenkelPoole-like at room temperature and beyond.

- The copper content and the irradiation do not change the conduction mechanisms, only the weight of the different contributions is shifted towards more variable-range-hopping conductivity.

- The activation energy at high temperatures is about $0.28 \mathrm{eV}$ for matrix and $0.25 \mathrm{eV}$ for tracks almost independent of $\mathrm{Cu}$ concentration.

- The activation energy at high temperature corresponds to results of other authors.

- The temperature dependence of the VRH component is very similar for tracks and matrix, as well as different copper concentrations. A tendency of the prefactor in the VRH formula to increase with $\mathrm{Cu}$ concentration is observed. The resulting $N\left(E_{\mathrm{F}}\right)$ are physically plausible.

- The weight of FP and VRH, indicated by the transition temperature, shifts to higher values for increasing $\mathrm{Cu}$ content and tracks, where VRH is more dominant.

- The temperature-dependent-activation energies (derivatives) support the transition from the VRH to the FP conduction at around room temperature.

- The curvature $(\beta)$ of the $I$ - $V$-characteristics is lower than suggested by the FP model, leading to unphysically high values of the calculated dielectric constant $\epsilon$. However, because of parallel mechanisms such as hopping, this behavior is 
expected. The curvature decreases at higher $\mathrm{Cu}$ concentrations and in case of tracks, where hopping is more dominant.

- The curvature of the track data is well described by Pollak and Riess moderatefield correction in the domain of VRH leading to plausible hopping ranges $R$. The hopping range decreases with increasing copper concentration, indicating an increasing number of hopping sites.

- The exponent $\gamma$ supports the description by FP and moderate-field correction.

The stretched exponential fitting and the explanation by FP as well as VRH are a successful method to describe the transport behavior of ta- $\mathrm{C}$ with and without $\mathrm{Cu}$ contaminations as well as tracks in a semi-classical empirical model. The temperature dependence can be reliably fitted in accordance to other experimental results. The field dependence is treated in a more empirical and qualitative manner because of the limitations of the theoretical models to describe field dependence correctly and the lack of microscopic knowledge of the structure. In order to understand the field dependence sufficiently well, a low-level simulation of the transport processes is most likely the best approach.

The semi-classical models applied allow only a limited interpretation of the microscopic effects of copper and tracks. Fitting results such as $\epsilon, N\left(E_{\mathrm{F}}\right)$ and $\alpha$ should only be considered rough estimates. The true microscopic structure requires quantum mechanical treatment and a better modeling of the random nature of the structure. However, the development of fitting parameters by the addition of copper and tracks give a basic understanding of the change in structure. Furthermore, this approach is suitable to model the current in ta-C and conductive ion tracks even with the addition of copper.

A simple microscopic description, supported by the fitting results, is a network of high- and low-resistivity links between neighboring atoms in the carbon matrix. An $\mathrm{sp}^{3}$ link (weak link) causes a high resistivity, while current passes easily through $\mathrm{sp}^{2}$ links. The overall conductivity of the ta-C matrix depends only on the ratio of $\mathrm{sp}^{2}$ to $\mathrm{sp}^{3}$ hybridization. In tracks the $\mathrm{sp}^{2}$ content is increased and the resistivity drops. The remaining weak links are passed dominantly by tunneling (hopping). The local field is very high at the remaining points of high resistivity leading to barrier saturation, even at low average fields (large barrier reduction according to FP). Hence, the behavior is more ohmic. Copper does not directly change the hybridization ratio of carbon at low concentrations. However, the copper clusters will favor $\mathrm{sp}^{2}$ bonds on its surface and will thereby short circuit weak links by adding additional paths around. Ultimately, the effect on conductivity is similar to the reduction of weak links by track formation. To some extend, both effects add up in case of tracks in ta-C:Cu. The effect of copper is decreased by the confined dimensions of the track allowing the introduction of new paths basically only along one dimension. At high $\mathrm{Cu}$ content the track formation is dampened by hindering material transport and 
absorption of thermal energy by the larger $\mathrm{Cu}$ clusters. This model, considering only the two hybridization states, would explain why only the ratio of the conduction mechanisms and their prefactors change while the temperature dependencies remain constant.

\subsection{Comparison of track and matrix conductivity}

Besides trying to understand and model the data of ion tracks in samples with different $\mathrm{Cu}$ concentrations, an optimal $\mathrm{Cu}$ concentrations should be found for increasing the track conductivity while keeping an insulating surrounding matrix. In this respect, the evaluation of the data will be done using the values of $\sigma_{0}(T)$. Two aspects are considered:

- High track conductivity and

- high ratio of track-to-matrix conductivity (track contrast).

The following graphs will benchmark the tracks with different copper concentrations using these two criteria on the basis of the fitted $\sigma_{0}(T)$ as this value gives a good indication of the conductivity of matrix and tracks. The comparison is made by calculating three ratios showing the increase in conductivity in the matrix, tracks, and the track to matrix contrast.

First, the impact of the copper on the matrix conductivity is portrait in figure 5.24. At the bottom the base-line of pure ta-C is at the ratio of one. The copper doping increases the conductivity, except for $0.1 \% \mathrm{Cu}$, the ratio increases with higher doping concentrations. While small concentrations of $0.5 \%$ cause only an increase of a factor 10 to 50, the highest doping of $5 \%$ increases the current by a factor of 300 to 10000 . All curves have a maximum at low temperatures between $120 \mathrm{~K}$ to $150 \mathrm{~K}$. At very low temperatures, the effect of the copper decreases in the regime of the residual conductivity decreasing the ratio. Towards higher temperatures, the ratio continuously decreases by an order of magnitude to room temperature, decreasing further to higher temperatures. Beyond the transition temperature of the VRH and FP regime, the ratio should be almost constant because the thermal activation is essentially independent of copper concentration. This constant ratio is seen for smaller $\mathrm{Cu}$ concentrations up to $2 \%$. The copper doping is therefore more advantageous at higher temperatures, where the increase in matrix conductivity is smallest.

Next, figure 5.25 shows the ratio of $\sigma_{\text {track }}$ to tracks in pure ta-C. Again, the baseline at one is given by pure ta-C. The copper doping increases the conductivity and thus the ratios. However, the increase is not as large as in the matrix measurements. Values are in the order of 1 to 6 . In addition, higher copper concentrations do not result in higher conductivities, a maximum is observed for about $0.5 \%$ to $1 \%$. At concentrations beyond $1 \% \mathrm{Cu}$, the enhancement of the track conductivity decreases again. The ratios are more or less temperature-independent. The strong rise of 


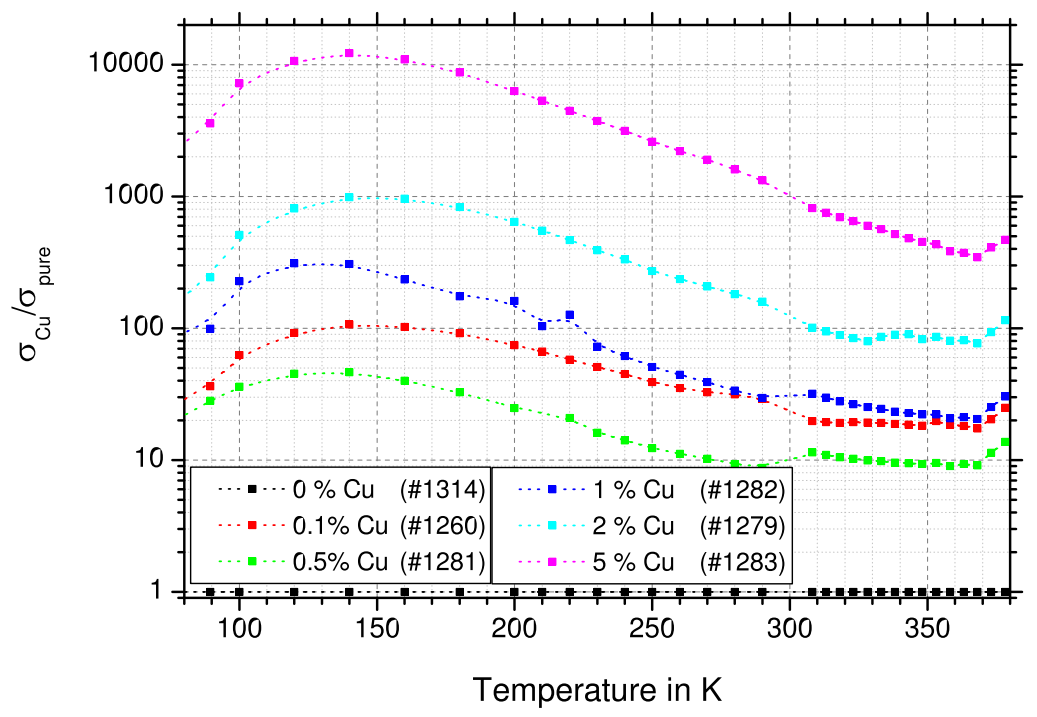

Figure 5.24: The increase of $\sigma_{0}(T)$ caused by copper in unirradiated ta-C:Cu. The effect of copper is largest at low temperatures in the VRH regime. Above room temperature, in the thermally activated regime, the effect is smaller. Copper impurities are more promising in high temperature applications.

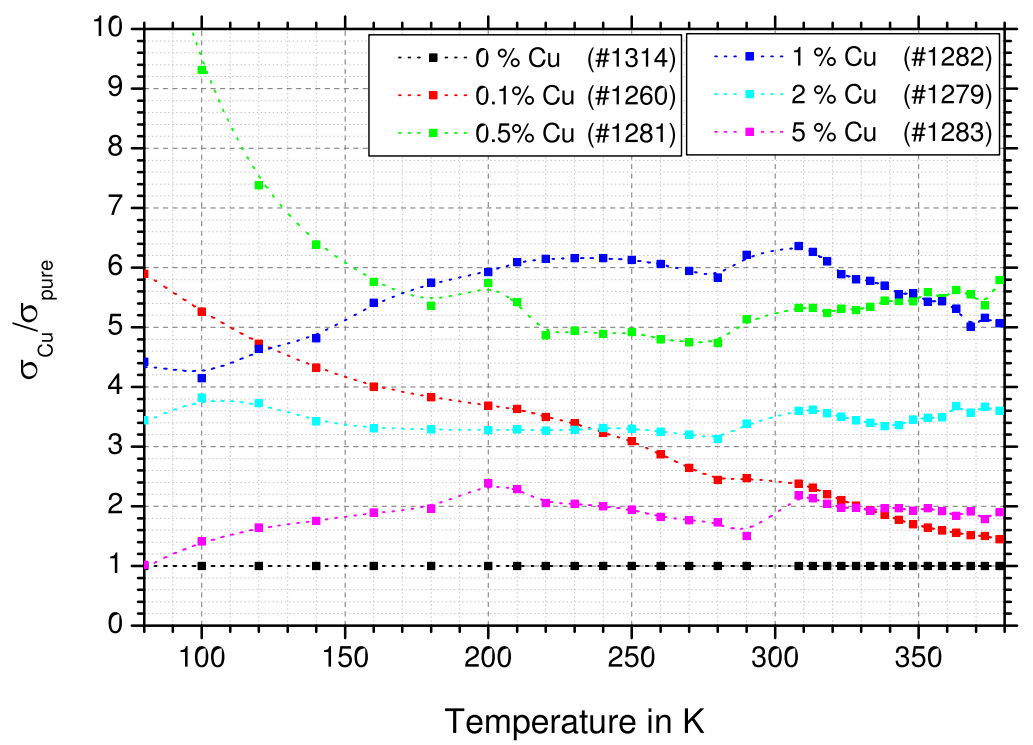

Figure 5.25: The increase of $\sigma_{0}(T)$ caused by copper impurities in ion tracks in ta-C:Cu. The increase in conductivity is smaller compared to the effect on the matrix. Furthermore, the best conductivity enhancement is achieved with $1 \% \mathrm{Cu}$. Higher concentrations do not increase the transport as much. The increase in conductivity is only slightly temperature-dependent. The increase of $0.5 \% \mathrm{Cu}$ al low temperatures is caused by a higher residual conductivity. 


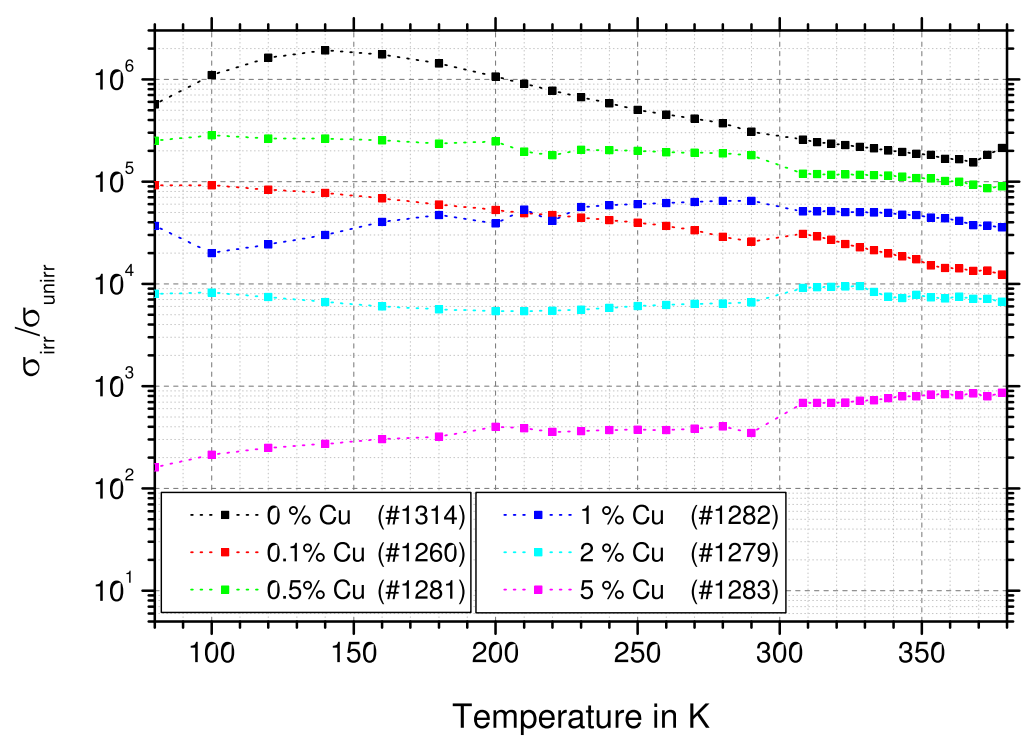

Figure 5.26: The $\sigma_{0}(T)$ contrast of tracks and matrix depending on $\mathrm{Cu}$ concentration. Pure ta-C has the highest contrast, followed by $0.5 \%$ and $1 \% \mathrm{Cu}$. At room temperature and beyond, the difference of the contrast of small copper concentrations compared to pure ta-C is small. Here, $\mathrm{Cu}$ could be used to enhance track conductivity without destroying the contrast ratio.

the $0.5 \%$ curve is caused by a high residual conductivity. Only the sample with $0.1 \%$ behaves differently. This sample has already shown a much smaller activation energy resulting in the decreasing ratio towards higher temperatures. Otherwise for other samples, a constant ratio is expected as the activation, as well as the VRH temperature dependence of tracks are very similar. The increase in track conductivity is almost temperature independent. Up to a concentration of $1 \%$, the conductivity is enhanced, beyond that concentration, the conductivity decreases. Tracks with $5 \%$ $\mathrm{Cu}$ show only twice the conductivity of pure ion tracks.

Finally, the track contrast is shown, these values are formed by $\frac{\sigma_{\text {track }}}{\sigma_{\text {matrix }}}$ and displayed in figure 5.26. The pure ta-C matrix has the highest contrast. The tracks are most pronounced. The irradiation increases the conductivity by more than five orders of magnitude. At very low temperatures the residual conductivity reduces the contrast, at about $150 \mathrm{~K}$ a maximum is reached, here the contrast surpasses one million. Towards higher temperatures, the contrast decreases and stabilizes around room temperature. The copper doping reduces the contrast in all cases. With the exception of $0.1 \% \mathrm{Cu}$, the reduction in track contrast correlates with impurity concentrations. In case of the $5 \% \mathrm{Cu}$ sample, the contrast is reduced to a factor of less than 1000 . On the other hand, $0.5 \% \mathrm{Cu}$ reduces the contrast only at temperatures below $300 \mathrm{~K}$, otherwise, it is almost on pair with pure ta-C. Doping the ta-C with $1 \%$ copper reduces the ratio at low temperatures by a factor of about 100 compared to pure ta-C. However, at room temperature, the difference is about a factor of four. 
Higher copper concentrations reduce the track contrast strongly. The copper doping is more efficient at higher temperatures in terms of track contrast. This effect is caused by the constant thermal activation in all samples at sufficiently high temperatures. Concentrations producing the best enhancement are in the order of $0.5 \% \mathrm{Cu}$ and $1 \% \mathrm{Cu}$.

In conclusion, $0.5 \%$ and $1.0 \% \mathrm{Cu}$ are most promising. The absolute increase in track conductivity and the relative contrast ratio are best for these concentrations. The contrast is best at room temperature and beyond in the thermally activated regime.

Physically, the small temperature dependence of the conductivity enhancements of the tracks (figure 5.25) supports the conclusions of the zero-bias conductivity predicting no change in the overall conduction model. Instead, the prefactor (amount of hopping sides and states) increases enhancing the transport. The ratios of the matrix conductivities (see figure 5.24) show a temperature dependence caused by the shift in transition temperature of the thermally activated to the VRH transport. This shift also causes the temperature dependence of the track to matrix ratio of pure ta-C seen in figure 5.26. All other samples show less change in current contrast over temperature because the thermal activation and VRH temperature dependence as well as the transition temperature are relatively constant for matrix and tracks.

In the regime of tested $\mathrm{Cu}$ concentrations, the matrix conductivity increases with more $\mathrm{Cu}$ impurities. At the highest concentration, a shift towards more $\mathrm{sp}^{2}$ hybridization has been observed suggesting a continuing of the trend. The track conductivity is already at a maximum at about $1 \% \mathrm{Cu}$. Nonetheless, all $\mathrm{Cu}$ concentrations increase the track conductivity compared to pure ta-C tracks. This behavior is most likely governed by the cluster size of the metal. At low concentrations of up to $1 \%$ no visible clusters could be found in ta-C suggesting sizes of $1 \mathrm{~nm}$ to $2 \mathrm{~nm}$. At $5 \% \mathrm{Cu}$ clusters are visible in TEM [153]. Within the confined track volume, the number of clusters is probably more important for track conductivity than the size. At higher concentrations, larger clusters form with radii in the order of 5 nanometer [156]. In addition, the track formation depends on thermal transport capabilities of the target. In case of high thermal transport the required stopping power increases. Larger copper cluster could spread the ion energy to a larger volume decreasing the completeness of the track formation process. In addition, the larger track may hinder the material transport (hillock formation) causing higher pressures during the track formation process. Again, the result could be less complete track formation.

\subsection{Scalability of ensemble measurements}

The evaluation of data measured on track ensembles with macroscopic contact pads is based on the assumption of individual separated tracks, which linearly add to the conduction. The evaluation of $\sigma_{0}(T)$ is based on this linearity. 


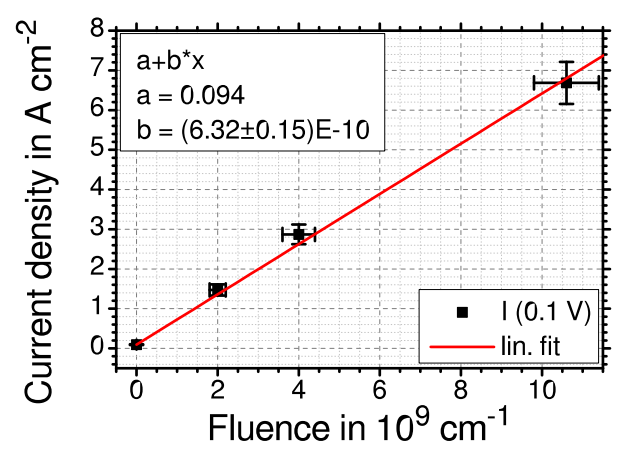

Figure 5.27: The current at $300 \mathrm{~K}$ and $0.1 \mathrm{~V}$ for unirradiated and differently irradiated ta-C films. The increase is linear, as expected. The linear fit has a y-intercept of $0.094 \mathrm{~A} \mathrm{~cm}^{-2}$ and a slope of $6.32 \times 10^{-10}$ A per ion.

Figure 5.27 shows the relation of fluence and current density of all parts of sample \# 1260. For this sample three different fluences were applied and measured. The irradiation density was measured by AFM analysis. The error-bars in x-direction represent the statistical uncertainty of the fluence evaluation. The error-bars in ydirection demonstrate the spread of different contact pads on a single sample (film inhomogeneity). The linear fit demonstrates the expected linear relation within the margin of error. The slope of the fit function $6.32 \times 10^{-10}$ A per ion at a bias potential of $0.1 \mathrm{~V}$. As expected from the $\sigma_{0}(T)$ evaluation the resulting conductivity per track is $8.1 \mathrm{~S} \mathrm{~m}^{-1}$ assuming a diameter of $8 \mathrm{~nm}$, a length of $65 \mathrm{~nm}$, and ohmic behavior.

In a second step, the scalability is tested at different temperatures. The specific track conductivity $\sigma_{0}$ is plotted for all fluences in comparison in figure 5.28. Ideally, all functions should superimpose each other. However, some temperature-dependent divergence can be observed. At very low temperatures, the residual conductivity, independently of track behavior, is dominant. Between $50 \mathrm{~K}$ to $200 \mathrm{~K}$, the lowest fluence shows twice the conductivity expected by the other two measurements. Towards room temperature, the scaling works very well. Finally, at high temperatures, the slopes are noticeable different. This particular sample was contaminated by a small copper concentration of less than $0.1 \%$. The impurity distribution is not necessarily homogeneous in all parts of the sample, possibly causing some deviations. Secondly, the films show a certain inhomogeneity in film thickness. At lower temperatures, where characteristics are far from ohmic, the false assumption of film thickness and resulting false fields have an exponential effect on calculated specific conductivity being most likely the reason for the deviation at lower temperatures. Finally, the effect of track-overlap could be responsible for spread of the results. This effect could be even temperature-dependent because ta- $\mathrm{C}$ hit twice by ions forms especially high conducting tracks. On the other hand, the chance of double-hits at $1 \times 10^{10} \mathrm{~cm}^{-2}$ is about $1 \%$ and only $0.25 \%$ of the total track area is hit twice. Therefore, the effect of double hits should not be dominant at the fluences evaluated.

In conclusion, it should be noted, that scaling of the conductivity works sufficiently well. The main limitations are film inhomogeneity and uncertainties of the actual ion fluence. The fluence has been measured by AFM analysis and is thereby known 
to reliable precision. Therefore, the main source of error is the inhomogeneity in impurity concentration and film thickness. The conductivity of tracks, measured by $\mathrm{AFM}$, is also compared to the track ensembles in order to validate the normalized values of the ensemble measurements (chapter 6).

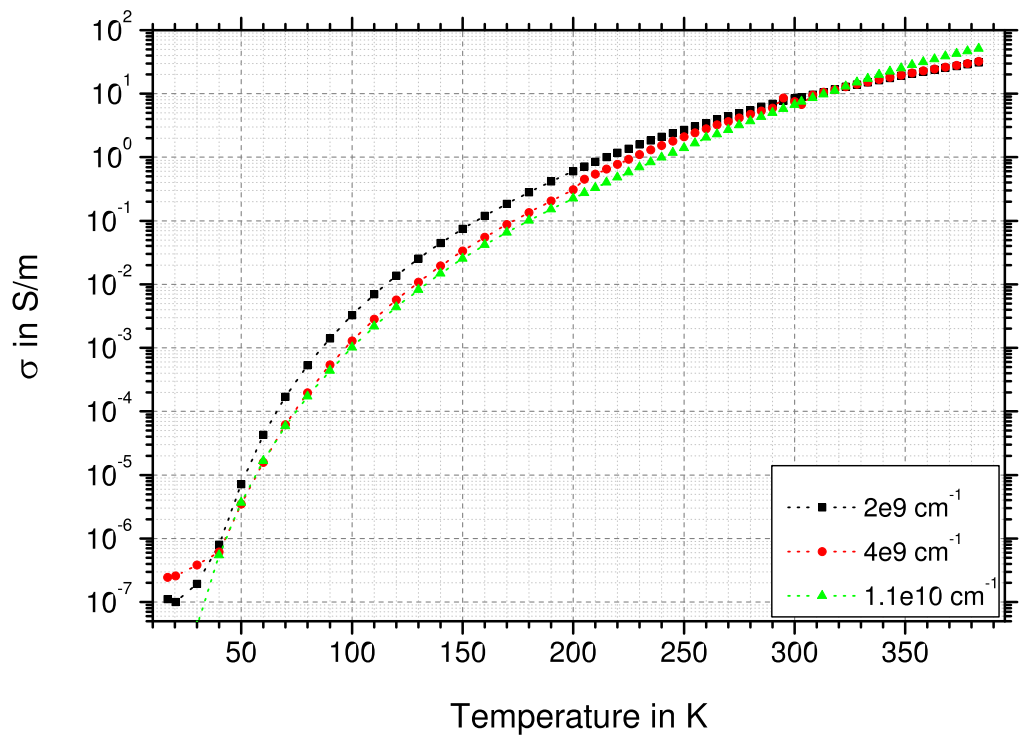

Figure 5.28: The zero-bias conductivity $\sigma_{0}(T)$ for ta-C irradiated with three different fluences. The different contact areas due to the different fluences were taken into account. At low temperatures, different residual conductivities are observed towards room temperature, the calculated values for $\sigma_{0}(T)$ are comparable. Only the values of the lowest fluence are higher around $150 \mathrm{~K}$. At room temperatures, all fluences yield the same conductivity. Towards higher temperatures, the slopes are different.

\subsection{Residual Conductivity and stability of films}

The residual conductivity is the temperature independent conductivity at low temperatures limiting the maximal resistance of the films. In $I-T$-plots, this behavior is visible as plateaus at low temperatures as shown, for example, in figure 5.29 very distinctively.

Generally, this type of residual conductivity is not unusual for thin amorphous films. Sze [179 and Sullivan et al. [180] measured residual conductivity in $\mathrm{SiN}_{\mathrm{x}}$. The typical explanation for this transport mechanism is nearest neighbor hopping of shallow traps at low fields. This process requires only a very small activation energy and is mainly dominated by tunneling. In the context of VRH and the Miller-Abrahams conduction (equation 2.35), the $2 \alpha R$ term dominates. At higher 
fields, the barrier lowering of shallow traps as illustrated by the FP model, can be sufficient to free an electron. At $30 \mathrm{~K}$, the thermal energy is $2.5 \mathrm{meV}$. In comparison, $1 \times 10^{7} \mathrm{~V} \mathrm{~m}^{-1}$ lowers the potential of an electron over a distance of $0.25 \mathrm{~nm}$ by the same energy. In other words, a level thermally activated at $30 \mathrm{~K}$ is activated as well by a field of $1 \times 10^{7} \mathrm{~V} \mathrm{~m}^{-1}$ at lower temperatures. Another high field effect is the propagation of hot electrons. The electrons gain significant energy over relatively small hopping distances. This kinetic energy can be transferred to a trapped electron, which in turn migrates increasing the transported current. Ultimately, at high fields, the electron temperature can be above the lattice temperature. The transport of hot electrons has been described, for example, by O'Dwyer [181. He suggests a $J \propto \exp (\sqrt{F})$ relationship, which is obtained as well for FP and Schottky conduction.

The current density in the residual conductivity regime depends on local film properties and varies significantly by position and sample. Figure 5.29 shows an example of the variation of the plateau in case of four contacts on the same ta-C film. The residual conductivity varies by three orders of magnitude. At high temperatures, all measurements show the same current, the thermally activated transport at all positions is comparable. The plot shows a greater dominance of the residual conductivity at higher fields supporting the concept of field induced hopping (activation) and hot electrons. The tunnel processes strongly depend on local DOS distributions and similar to the concepts of VRH, the assumption of few highly conductive paths is reasonable. The quality of these paths depends on statistical distribution but also on sample quality and treatment.

A main aspect in terms of sample quality is roughness of the substrate. The film shown in figure 5.29 was grown on ultra-flat $\mathrm{Si}(<0.3 \mathrm{~nm}$ RMS). However, the substrates were cut and handled in non-clean-room environments. The cleaning process for the substrate used for this earlier film was different compared to all other samples shown in this work. The silicon was wiped off with laboratory paper just before mounted in ADONIS. This procedure resulted in small particles on top of the substrate. Later, substrate cleaning was finished by blowing off excess solvents with $\mathrm{N}_{2}$-gas. This measure resulted in much less pronounced residual conductivities. The cleanness of the substrate varies by sample position explaining the spread of the plateaus measured on a single sample.

The correlation of substrate roughness and residual conductivity is experimentally evident looking at measurements of ta-C films on metals. Nix et al. measured the temperature-dependent conductivity of ta-C ion tracks on $\mathrm{Ni}[33$ as well as $\mathrm{Fe}[32$. The dynamic range of the currents over temperature are much smaller in case of metal substrates than silicon. Nix gave a RMS roughness of $3.22 \mathrm{~nm}$ for the Fe substrate. The results of a comparable experiment are shown for a $\mathrm{Cr}$ substrate $(50 \mathrm{~nm}$ evaporated onto $\mathrm{n}-\mathrm{Si}$ ) in figure 5.30. The current at $0.1 \mathrm{~V}$ of two track ensembles (a) and (b) on $\mathrm{Cr}$ are compared to a track ensemble in ta-C directly deposited onto silicon. At temperatures above $250 \mathrm{~K}$, all three pads show a comparable behavior. At lower temperatures, the slope of the current is much smaller on the rougher $\mathrm{Cr}$ 


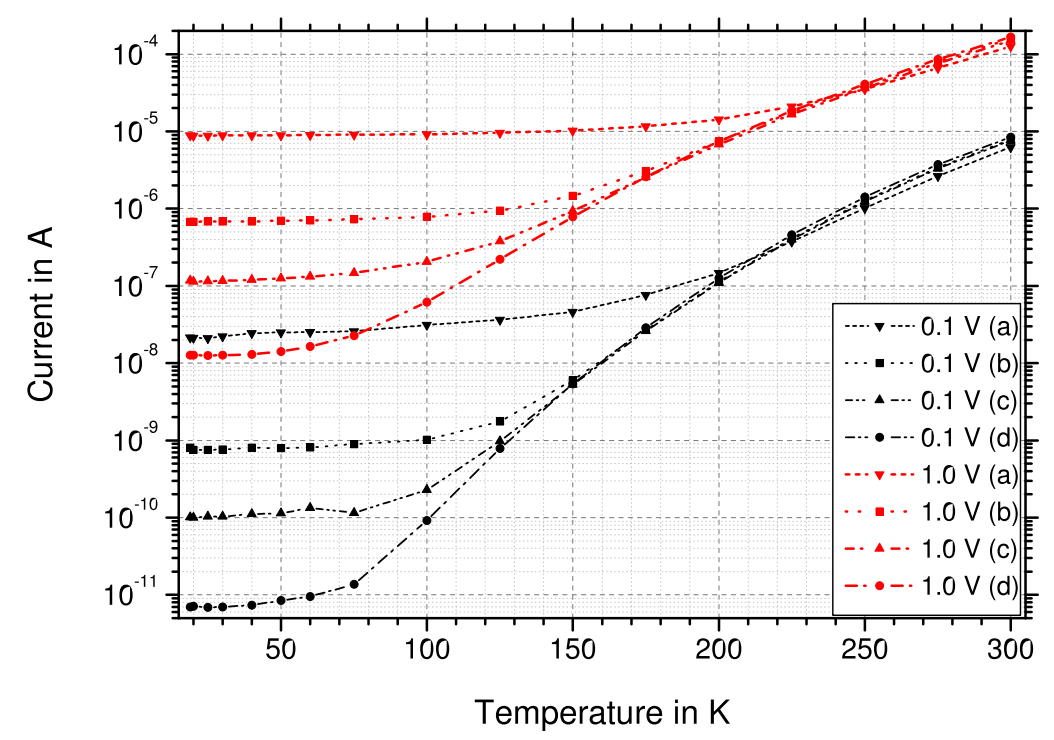

Figure 5.29: $I$ - $T$-characteristics $(0.1 \mathrm{~V}$ and $1.0 \mathrm{~V})$ of different contact pads on unirradiated ta-C film. At high temperatures, all curves are comparable, at low temperatures, a strong fluctuation of the residual conductivity is observed. The elevated plateau is attributed to unclean substrate surfaces during the deposition process.

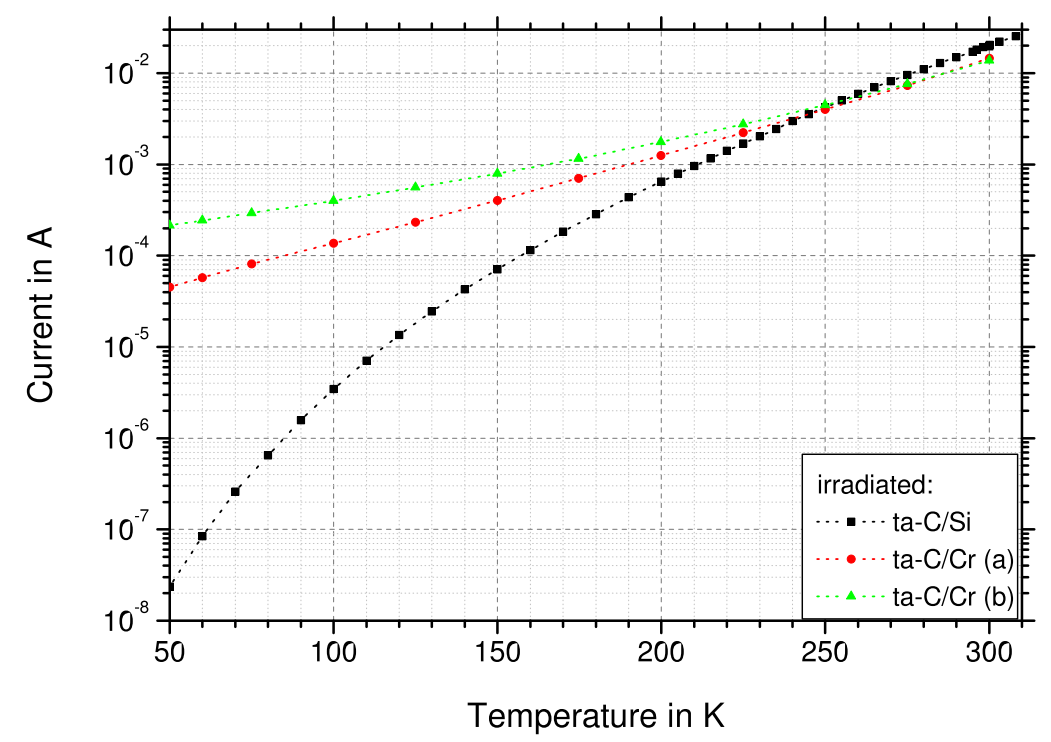

Figure 5.30: $I$ - $T$-characteristics $(0.1 \mathrm{~V})$ of ion track ensembles in ta-C films deposited on flat $\mathrm{n}$-Si ( $<0.3 \mathrm{~nm}$ RMS roughness) and on rougher $\mathrm{Cr}$ ( $3 \mathrm{~nm}$ RMS roughness). At high temperatures, all measurements show comparable results at lower temperatures, the residual conductivity is increased in case of films deposited on rougher Cr surfaces. 
substrate. Both pads (a) and (b) show different slopes and deviate towards low temperatures. The roughness of the Cr substrate was $3 \mathrm{~nm}$ RMS (AFM), which is very similar to the roughness of the substrates used by Nix. Contrary to the temperature independent plateaus of the unirradiated ta-C shown in figure 5.29, a slight temperature dependence remains in case of the track ensemble on metal substrates.

The roughness of the interface, induced by the type and cleanness of the substrate, influences the weight of the residual conductivity. One effect is the reduction of the film thickness locally at high spikes. These spikes may have an antenna-like effect injecting more hot electrons. Ta-C grows by subplantation and the behavior of the $\mathrm{C}$ ions beneath the surface is important. The sputtering and growing of the film during deposition reduces the roughness. Therefore, the sputtering and subplantation rates are locally different during the deposition process of the first few nm. Again, the effective film thickness, the hybridization ratio as well as the DOS can be affected by the roughness of the substrate. All films analyzed were between $50 \mathrm{~nm}$ to $100 \mathrm{~nm}$ a RMS roughness of a few $\mathrm{nm}$ is significant.

A further increase to the residual conductivity is attributed to applying too high fields to the sample and changing its characteristics permanently. In figure 5.31, the temperature dependent current of a ta-C:Cu film is shown for $0.1 \mathrm{~V}$ and $1.0 \mathrm{~V}$ for three measurements. First, the characteristics were measured to $1.2 \mathrm{~V}$ (1. run). The same pad of the film was measured again this time up to $2.5 \mathrm{~V}$ (2. run). The plot shows a deviation at low temperatures, the residual conductivity is more pronounced after the second run. Finally, new contact pads were connected on the film and measured up to $2 \mathrm{~V}$. The third line is an extreme case of this measurement, where the plateau is observed already close to room temperature.

This experiment demonstrates the possible destruction (permanent change) of the sample by applying high electric fields. The change is only present at lower temperatures (VRH regime). The thermally activated domain at room temperature and above is not effected. In figure 5.32, the $I-V$-characteristics are shown at $300 \mathrm{~K}$. The first and second run yield the same curve, even the third run, tremendously affected by the elevated residual conductivity, seems comparable. The only difference is a $30 \%$ asymmetry. This asymmetry is typical for the observed residual characteristics suggesting the increased importance of the charge carrier injection.

The residual conductivity is on one hand an indicator for substrate roughness (cleanness). Furthermore, changes in sample behavior induced by high applied fields are indicated by an elevated residual conductivity. The examination of the ta-C:Cu film data, shown in figure 5.31, indicates a delicate effect of high fields on sample behavior on the VRH dominated temperature regime. Baring this effect in mind, the fluctuation of the temperature dependence of the VRH fitted to $\sigma_{0}(T)$ could be caused by the field induced effect (see table 5.2 and table 5.3). The sensitive correlation to substrate roughness limits the choice of substrates for the growth of thin ta-C films with consistent electrical properties at low temperatures. 


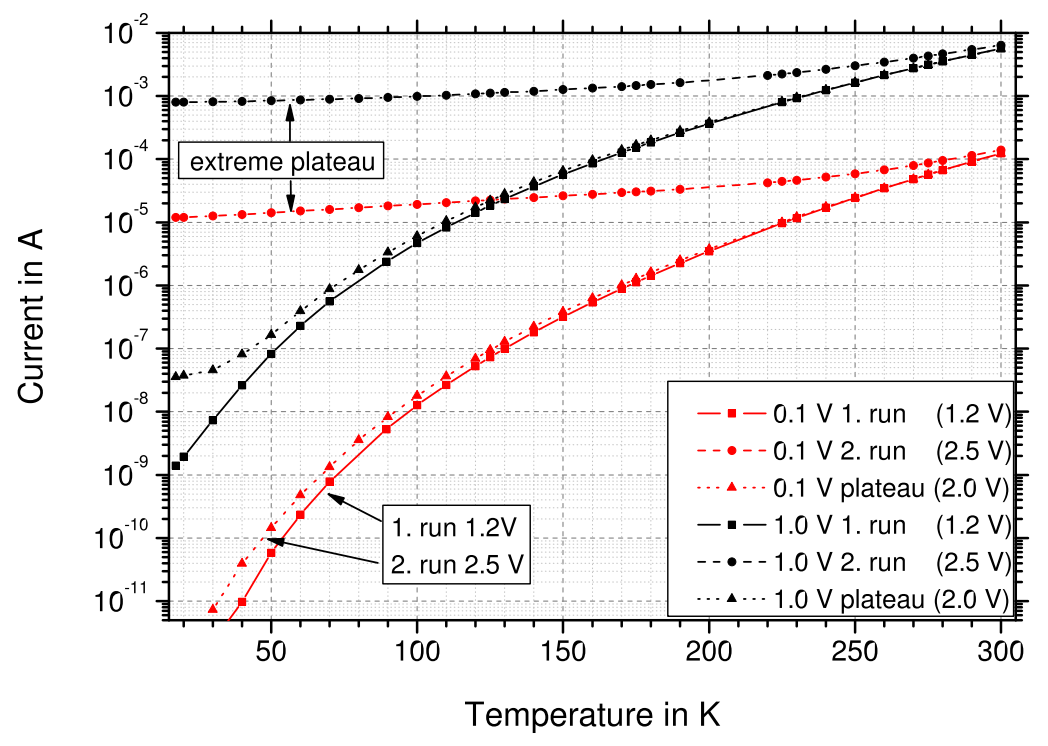

Figure 5.31: $I$-T-characteristics $(0.1 \mathrm{~V}$ and $1.0 \mathrm{~V})$ of three measurements on an unirradiated ta$\mathrm{C}: \mathrm{Cu}$ film. The first two runs use the same pad, the second run was performed to higher maximal potentials of $2.5 \mathrm{~V}$. At high temperatures, no difference is observed, at lower temperatures, the higher bias increased the residual conductivity. The third run (plateau) shows a different (new) pad measured up to $2 \mathrm{~V}$ at room temperature, the result is comparable to the first runs. At lower temperatures, an extremely high residual conductivity is observed.

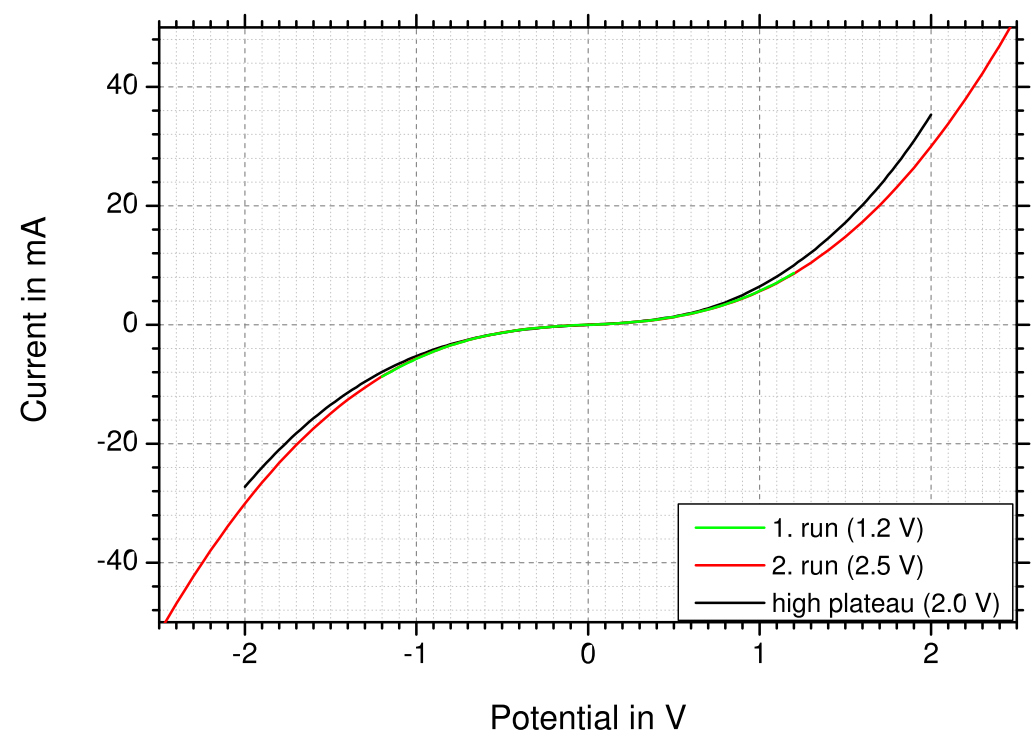

Figure 5.32: $I$ - $V$-characteristics at $300 \mathrm{~K}$ of pad measurements on an unirradiated ta-C:Cu film. The first and second run were performed on the same pad up to $1.2 \mathrm{~V}$ and $2.5 \mathrm{~V}$ respectively. No change is observed. The third run shows a measurement of different pad up to $2.0 \mathrm{~V}$ effected by an extremely high residual conductivity. Although similar to the first two runs, a noticeable asymmetry is visible. 


\subsection{Summary - contact pad characterization}

The characterization of pure ta-C has yielded comparable results to earlier experiments [27, 103]. The combination of the low- and high-temperature setup allows the analysis from $20 \mathrm{~K}$ to $380 \mathrm{~K}$. The resulting characteristics can be modeled by an extended FP model (chapter 5.5) beyond $200 \mathrm{~K}$. The irradiation increases the conductivity by orders of magnitudes as it has been observed in earlier publications 22, 25, 27, 32, 33. The characteristics become more ohmic at room temperatures and cannot be described efficiently by the FP model used to describe unirradiated ta-C. In this publication, the contact pads exhibiting very low resistivities (irradiated films and high $\mathrm{Cu}$ concentrations) were measured in four point geometry reducing the contact resistance significantly. In addition, the $\mathrm{Cr} / \mathrm{Au}$-contact pads show much better results, it is expected that Au-contacts in earlier publications 22, 25, 32, 33. led to reduced effective contact areas.

The addition of copper increases the conductivity of unirradiated and irradiated films. Interestingly, the overall temperature dependence is only slightly affected by the addition of copper. At high copper concentrations of $5 \%$, the effect of the irradiation becomes comparably small, the current increase is only small. The measured current is dependent on film thickness supporting a domination of the bulk ta-C instead of interface effect. Although, a slight asymmetry is observed in case of irradiated films with low resistivity suggesting a degenerated Schottky contact.

In order to describe the conductivities in matrices and tracks with different copper concentrations, a model based on a stretched exponential function was developed (chapter 5.6). This model is capable to fit the zero-bias conductivity $\sigma_{0}$ in terms of three conduction mechanisms. Despite the addition of tracks and copper impurities, the fitting results confirm the observation of an almost constant temperature dependence and describe $\sigma_{0}$ of the conduction mechanisms well between $20 \mathrm{~K}$ to $380 \mathrm{~K}$. Only the overall current increases. The curvature can be explained in terms of FP and moderate field correction of VRH. A more detailed summary of the results of this model is given in chapter 5.7 .

The comparison of the track conductivities depending on copper concentration have revealed an optimal concentration of $0.5 \%$ to $1 \%$ copper. Here, the track conductivity increases the most while the matrix conductivity increases only moderately persevering a high contrast of tracks and matrix. At higher copper concentrations, the current enhancement in tracks is reduced while the conductivity of unirradiated films increase further. Furthermore, this comparison showed the larger effect of copper on unirradiated ta-C compared to tracks.

In addition, the plausibility of the normalization of the track conductivity has been checked by comparing the results of different irradiation fluences (chapter 5.9). The linear dependency could be confirmed for fluences up to $1 \times 10^{10} \mathrm{~cm}^{-2}$. 
The residual conductivity, observed at low temperatures, has been linked to sample quality (chapter 5.10). In particular, the roughness and cleanness of the substrate increased the amount of residual conductivity. Furthermore, the possibility was shown to increase residual conductivity by applying too high electric fields. 


\section{Microscopic characterization of ta-C by AFM}

\subsection{Tracks and hillock}

As portrayed in chapter 2.3.7, individual tracks can be visualized by AFM in two ways. The topographic map of the film surface is covered with a hillock at each ion impact side and the current-mapping at a constant bias voltages shows the conductive tracks embedded in the insulating matrix. Figure 6.1 shows an example of a $80 \mathrm{~nm}$ ta-C film irradiated with $4 \times 10^{10} \mathrm{~cm}^{-2} \mathrm{U}$ ions with an energy of $1 \mathrm{GeV}$. Both maps recorded simultaneously display the same area using a bias of $0.2 \mathrm{~V}$ in contact mode.

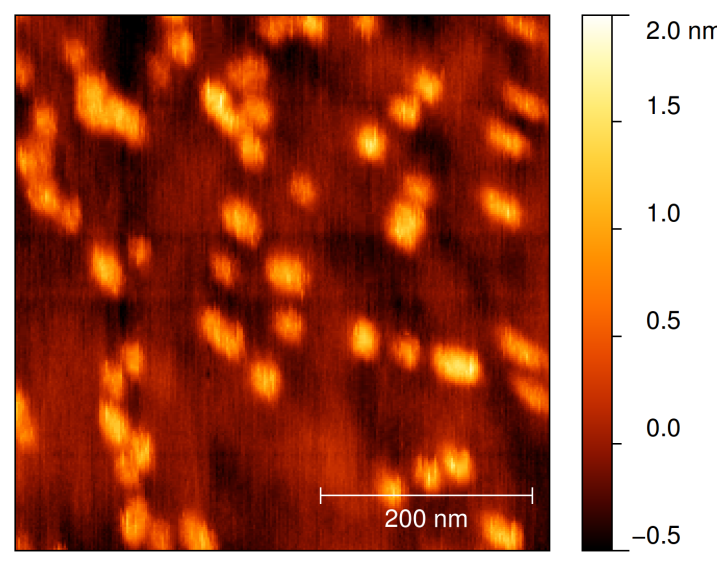

(a) Topography

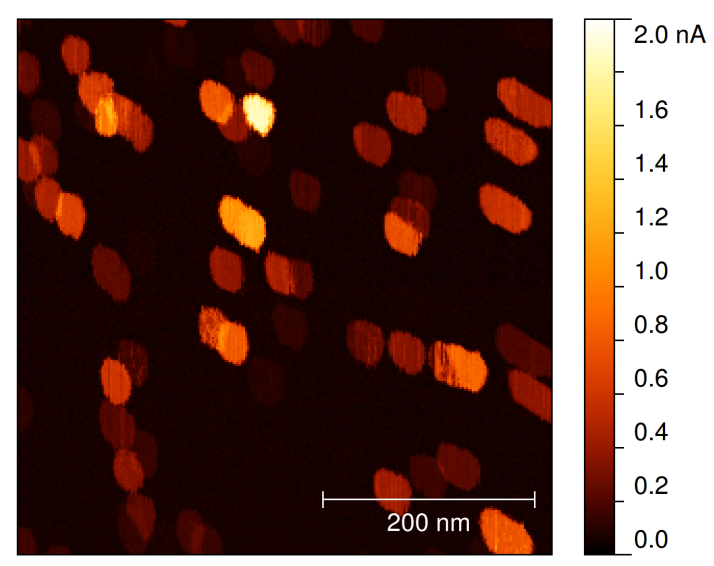

(b) Current-Map

Figure 6.1: AFM scans of irradiated ta-C film ( $1 \mathrm{GeV} \mathrm{U})$ where the topography (a) visualizes the hillocks of each ion track (bright spots). The current-map (b) (0.2 V) shows the increased current at each ion impact side. Both hillock height and current fluctuate significantly from track to track (variation of the brightness of the spots).

The landscapes recorded by the AFM tip are convolutions of the actual surface and the tip used. An idealized spherical tip can be approximated with a Gaussian convolution. The diameter of the hillock structures appears wider than expected by TEM analysis and theoretical calculations. The topographic hillocks in figure 6.1(a) seem to be about $30 \mathrm{~nm}$ wide, which is three to four times more than expected. In addition to the physical limitations of the tip, electronic noise interferes with the recording of the topography. Less electronic background noise and a finer tip result in smaller hillock diameter as seen in figure 6.2. In this case the diameter appears to 
be about $15 \mathrm{~nm}$, which is still about twice the expected radius. While the topographic convolutes with a soft function (e.g. Gaussian), the current convolution appears to be with a step function. As soon as the conductive part of the tip is in contact with a track, the maximum current flows. If the tip contacts two tracks simultaneously, the accumulative current is measured. The current suggests a track diameter of about $35 \mathrm{~nm}$. Therefore, AFM is not adequate to determine the track diameter to sufficient precision.

The ta-C films deposited on Si substrate are extremely flat with a RMS roughness of less than $0.09 \mathrm{~nm}$ (unirradiated). The apparent roughness is partially caused by the technical limitations of the setup (noise). Slight line shifts (vertical lines) are visible along the slow scanning axis. In case of figure 6.1 and figure 6.2 the slow axis is along the $\mathrm{x}$-direction. All images corrected have been adjusted by line medians and in some extreme cases, scars have been removed by gwyddion. Finally, an average quadratic background was removed excluding the hillock areas flatting and calibrating the height (the average background height is adjusted to be zero). The data of the current-maps was not corrected.

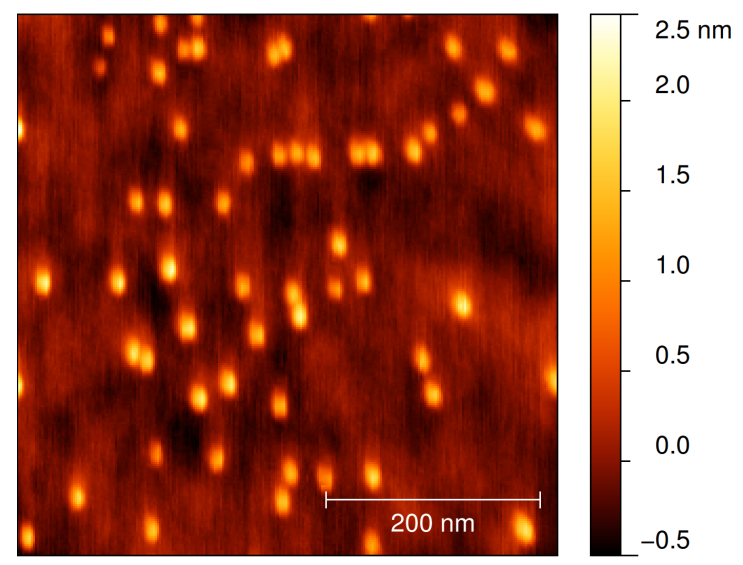

Figure 6.2: AFM topography of irradiated ta-C recorded with less noise in the electronic signal of the topography scan than figure 6.1(a) The hillocks appear to be higher but smaller in diameter, although measured on the identical film.

To treat the data in a quantitative manner, the hillock heights and the conductivities (normalized) currents are averaged for all tracks recorded by AFM. Furthermore, in order to analyze the fluctuation, histograms are calculated. To reduce human bias, all identifiable tracks of an AFM image were used 1 . The data was extracted using the mask tool of gwyddion.

The results for Uranium tracks in pure ta-C are shown in figure 6.3. The conductivity was calculated assuming cylindrical tracks with $8 \mathrm{~nm}$ diameter. The average conductivity of $3.4 \mathrm{~S} \mathrm{~m}^{-1}$ corresponds well to the result of the macroscopic measure-

\footnotetext{
${ }^{1}$ Tracks not completely recorded are account if atleast half the hillock is within the picture.
} 
ment of $3.5 \mathrm{~S} \mathrm{~m}^{-1}$. As suggested by the false color current-map (figure 6.1(b)), the distribution of track conductivities is wide. The data fits a Poisson-like distribution that could be explained by the concepts of hopping and the incomplete track formation (chapter 2.3.6). The quality of hopping sides depends on the randomly degree of material transformation inside the track volume. In a simple model, tracks can be split in short segments with dimensions in the order of typical hopping ranges. Each segment has a probability to be a good segment with low resistivity, otherwise, a high resistivity is assigned. Physically, the reason for either high or low resistivity of a track segment would be mainly influenced by the local ratio of $\mathrm{sp}^{2}$ and $\mathrm{sp}^{3}$ bonds of the carbon. An uninterrupted chain of $\mathrm{sp}^{2}$ bonds would provide a low resistivity while interruptions by $\mathrm{sp}^{3}$ links would increase the resistivity. In case of $80 \mathrm{~nm}$ long tracks and hopping ranges in the order of $5 \mathrm{~nm}$, only 16 segments would constitute each filament leading to a Poisson distribution rather than Gaussian if low overall conductivities are favored.

The hillocks show a Gaussian distribution around $1.4 \mathrm{~nm}$ with a FWHM of $1 \mathrm{~nm}$. The distribution of hillock heights is slightly widened by the surface roughness of the sample. The heights measured are in relation to the average background. The mean height is slightly smaller but comparable to the results presented by Krauser et al. 24]. Schwen et al. 23 measured hillock heights of about $4 \mathrm{~nm}$ ( $\mathrm{Pb}$ ions). Their larger values for hillock heights can possibly be explained by different film properties ( $\mathrm{sp}^{3}$-ratio) and a different processing of the AFM data. The average hillock height of $1.4 \mathrm{~nm}$, presented in this work, is smaller than expected by later results obtained by measurements of $\mathrm{U}$-irradiated ta-C: $\mathrm{Cu}$ films and by $\mathrm{Pb}$-irradiated ta-C. The difference is caused by electronical noise superimposing the topography signal. The AFM analysis of the ta-C films presented above in figure 6.1 and figure 6.2 was conducted before improving the electronic setup. All other samples were characterized after the reduction of signal noise. The critical parts of the AFM were protected with isolation transformers and the topography signal was passed through a low-pass filter. Therefore, the difference of measured hillock heights before and after upgrading the electronics cannot be compared. 

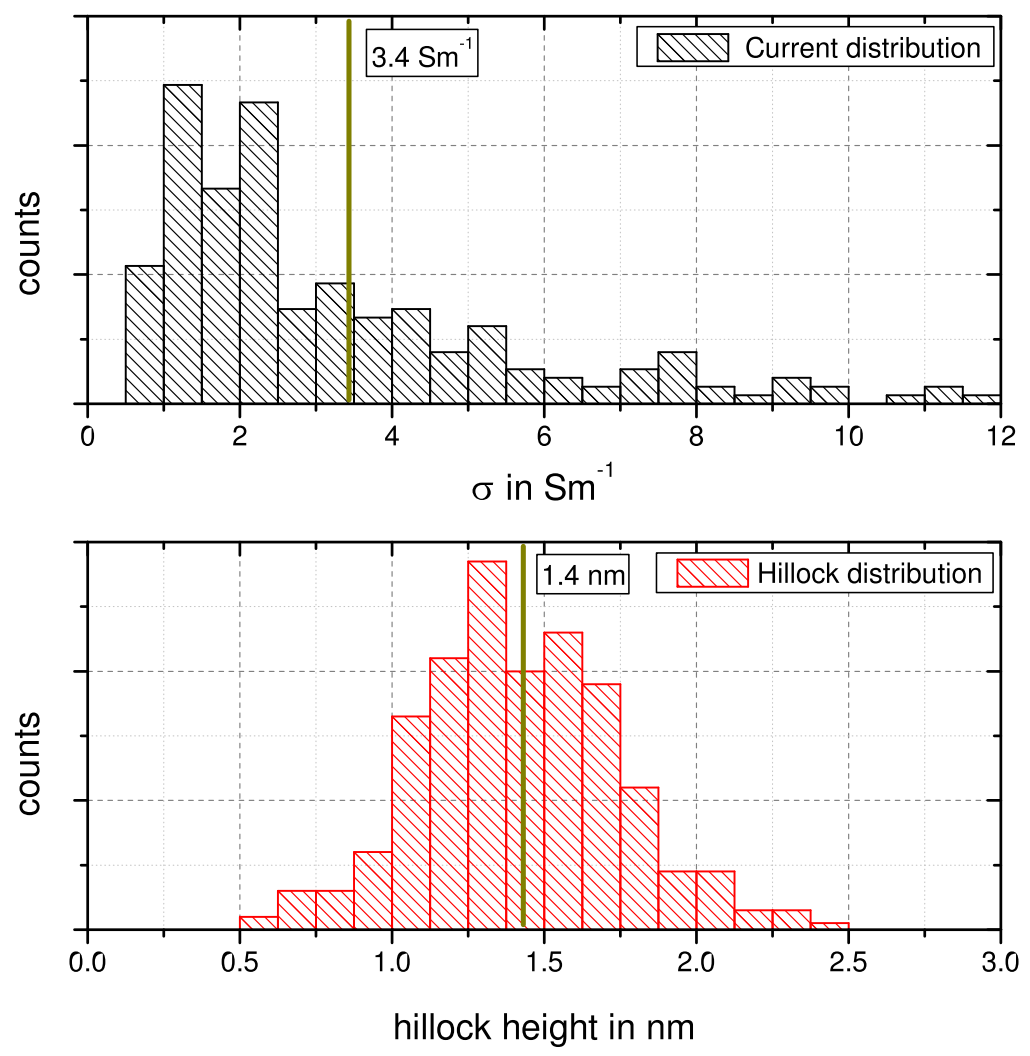

Figure 6.3: Histograms of track conductivity and hillock height measured by AFM on irradiated ta-C $(1 \mathrm{GeV} \mathrm{U})$. The conductivity appears to be a wide Poisson distribution with a mean value corresponding well to macroscopic measurements. The hillock height is Gaussian distributed around $1.4 \mathrm{~nm}$ which is slightly lower than reported by Krauser et al. 24 caused by instrumental differences.

\subsection{Effect of copper}

The irradiated ta-C:Cu films examined by macroscopic contact pads have also been analyzed by AFM. In figure 6.4, examples for the topography of all copper concentration up to $2 \%$ are displayed. The hillock height decreases with increasing copper concentration. The density of hillocks corresponds to the applied fluence. At $5 \%$ $\mathrm{Cu}$ no distinct hillocks were found (figure 6.6). The large white spots are surface contaminants on top of the film and not hillocks.

In figure 6.5 the current-maps of ion tracks in ta-C are shown for different $\mathrm{Cu}$ concentrations. All images were recorded using a bias of $0.3 \mathrm{~V}$. The track conductivity increases significantly with copper impurities as already seen in macroscopic measurements. As demonstrated on pure ta-C, the position of hillocks and elevated currents correspond to each other. The fluctuation of track properties is quantitatively analyzed below. Again, at $5 \% \mathrm{Cu}$ no obvious signs of tracks could be found. Comparing tracks in figure 6.5 and figure 6.1(b) reveals a different geometry of the 


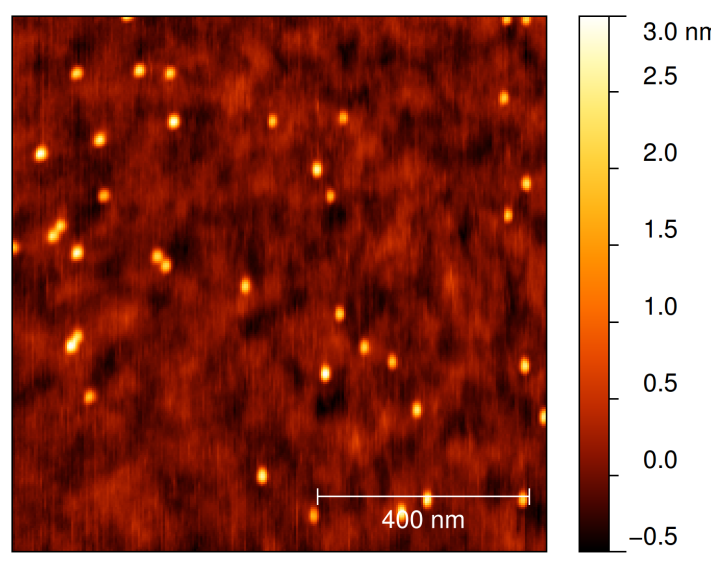

(a) Topography $0.1 \% \mathrm{Cu}$

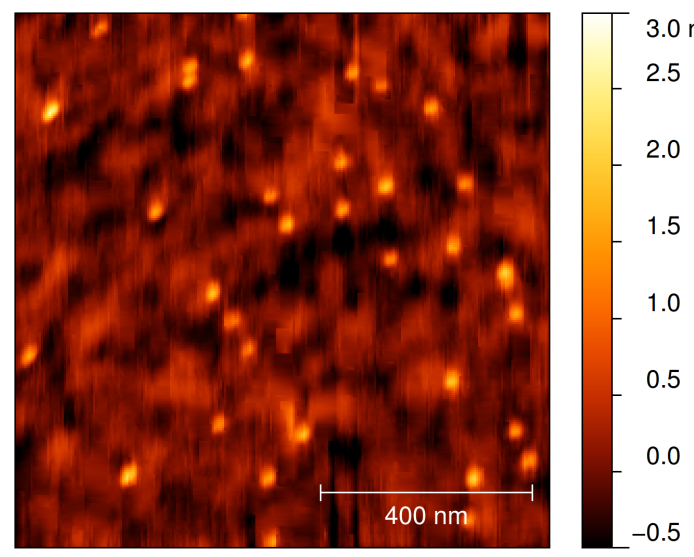

(c) Topography $1 \% \mathrm{Cu}$

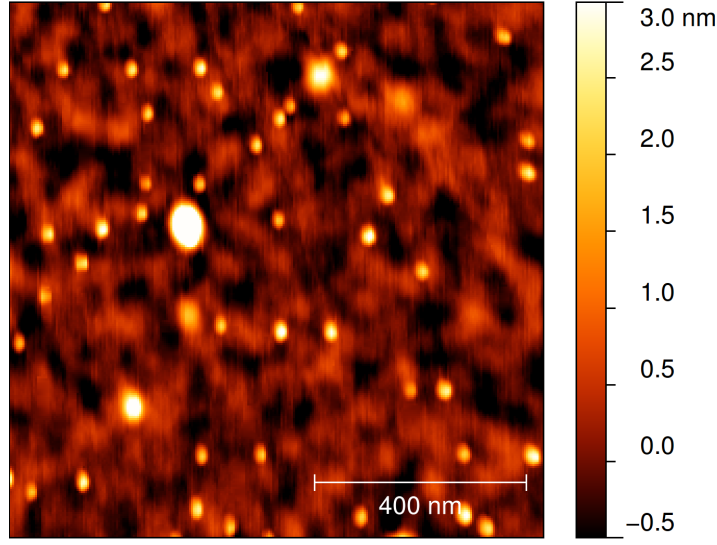

(b) Topography $0.5 \% \mathrm{Cu}$

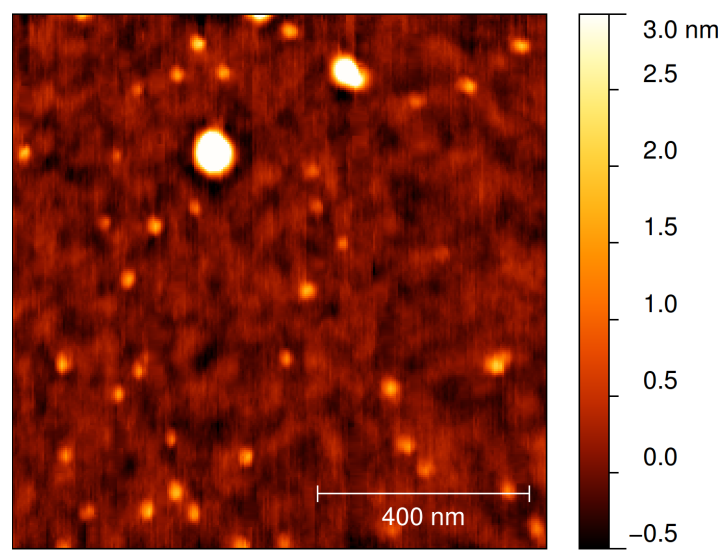

(d) Topography $2 \% \mathrm{Cu}$

Figure 6.4: AFM topography of hillocks on top of irradiated ( $1 \mathrm{GeV} \mathrm{U})$ ta-C:Cu films with different $\mathrm{Cu}$ concentrations showing a decrease in hillock height for high copper concentrations.

track signals. The later is more asymmetrical along the fast scanning axis caused by a slightly different type of AFM tip (MULTI75E). The offset in hillock height between films with low $\mathrm{Cu}$ concentrations and pure ta- $\mathrm{C}$ is caused by the instrumental improvements reducing the noise of the topographic signal. All ta-C:Cu films were measured under the same instrumental conditions using the same tip type, therefore, the results can be compared.

The data of the $5 \% \mathrm{Cu}$ film is displayed in figure 6.6. The topography (figure $6.6(a)$ shows an increased overall roughness compared to films with lower $\mathrm{Cu}$ concentrations. However, no explicit hillocks can be identified. The $\mathrm{Cu}$ impurities tend to accumulate at the film surface. The XPS measurements suggested a surface concentration of almost $10 \% \mathrm{Cu}$. In addition, the XPS experiments have shown 


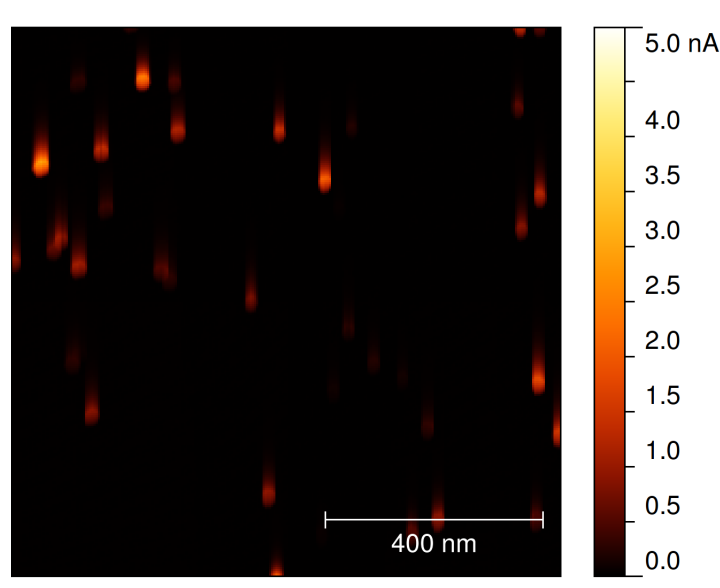

(a) Conductivity $0.1 \% \mathrm{Cu}$

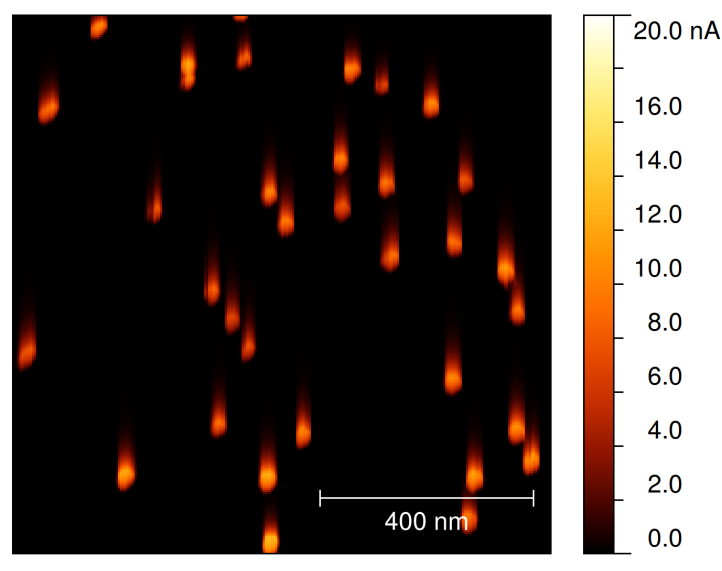

(c) Conductivity $1 \% \mathrm{Cu}$

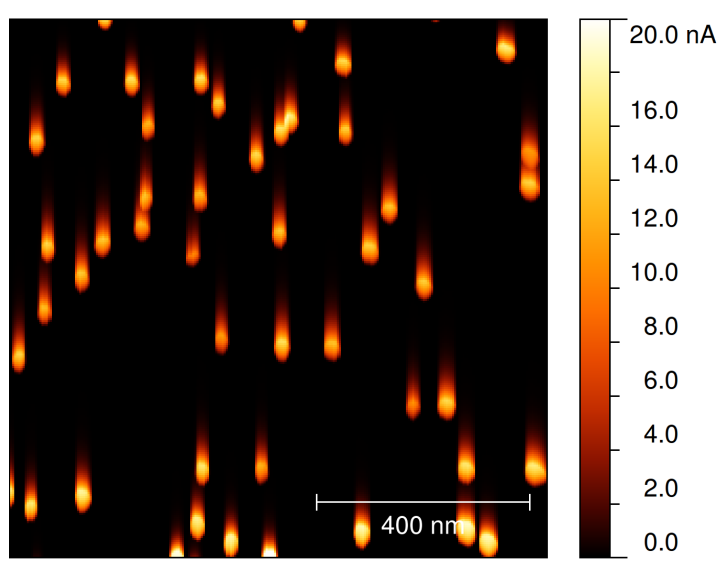

(b) Conductivity $0.5 \% \mathrm{Cu}$

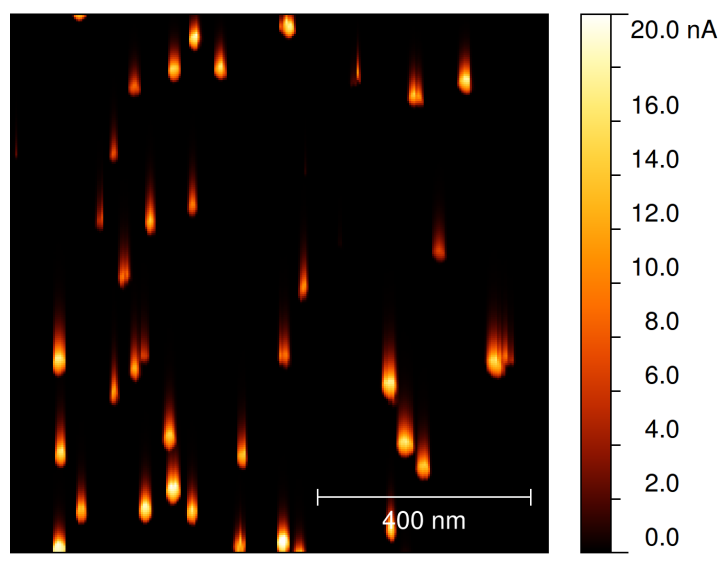

(d) Conductivity $2 \% \mathrm{Cu}$

Figure 6.5: AFM current-maps $(0.3 \mathrm{~V})$ of irradiated ta-C:Cu $(1 \mathrm{GeV} \mathrm{U})$ with different copper concentrations. The current increases significantly with higher copper concentrations.

a decreasing $\mathrm{sp}^{3}$ hybridization of the ta- $\mathrm{C}$ with high impurity concentrations. The hillocks are formed by the transformation from $\mathrm{sp}^{3}$-rich to $\mathrm{sp}^{2}$-rich a-C, if the initial ratio is shifted towards the graphitic structure, the hillock formation is reduced. Furthermore, the accumulating $\mathrm{Cu}$ cluster at the surface dampens the track formation as well. The clusters may absorb significant portions of the stopping power and hinder the material transport suppressing good hillock formation. Last, the initial roughness of the surface superimposes possible small structures rending possible small hillocks and random roughness indistinguishable.

The current-map (figure 6.6(b) also differs significantly compared to the irradiated surfaces presented before. Although some spots exhibit higher conductivities, they do not necessarily correspond to ion tracks. First, the conductivity is smaller than ex- 
pected from other copper concentrations and macroscopic measurements. Tracks are expected to show at least a conductivity 100 times higher than the matrix. Secondly, the number of conductive spots does not correlate with the irradiation fluence. The sample was irradiated along with the other ta-C:Cu films and should have obtained a similar irradiation fluence. The geometry of the conductive structures is significantly different, especially the width is much reduced compared to track signals in other current-maps. In fact, no two neighboring lines along the fast scanning direction (y-axis) show increased conductivity at the same y-position. These conductive scars indicate a sporadic and poor contact to the conducting spots. Therefore, the observed conductivity does not resemble the conduction caused by the ion tracks. The bad contact is caused by the overall roughness and the absence of distinct hillocks. The high number of copper cluster at the surface could present a false track signal by showing high conductivity while being touched by the tip. Finally, the samples were kept at air which could cause a surface oxidation of the metal which in turn could reduce the microscopic conductivity as well. Therefore, expected weak tracks are hidden to AFM analysis.

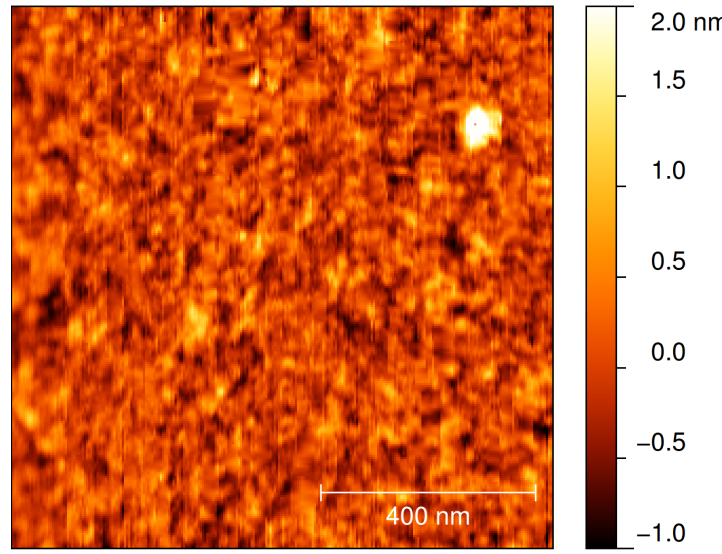

(a) Topography

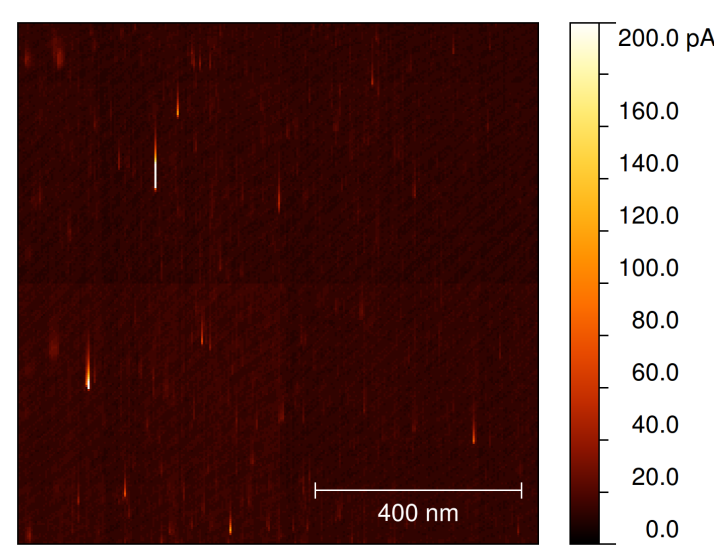

(b) Current-map

Figure 6.6: AFM scans of irradiated $(1 \mathrm{GeV} \mathrm{U})$ ta-C:Cu film with $5 \% \mathrm{Cu}$. The topography (a) shows an increased overall roughness compared to lower $\mathrm{Cu}$ concentrations. No distinct hillocks are visible. The bright spot is a dust particle. The current-map (b) (0.3 V) shows also no distinct tracks. Some spots of elevated conductivity are likely due to large $\mathrm{Cu}$ clusters.

For a quantitative analysis, all track data has been summarized in histograms. For this data pool, multiple AFM images of different areas were used for each sample. The data processing of the images was again accomplished with gwyddion.

In figure 6.7, the hillock height distributions are compared and the arithmetical averages are marked. The heights are distributed in Gaussian curve with about the same width for all copper concentrations. The width is also comparable to the distribution of tracks in pure ta-C. At Cu concentrations of $1 \%$ and $2 \%$, the hillock 
height drops by about $1 \mathrm{~nm}$. At $5 \% \mathrm{Cu}$ no distinct hillocks are visible (figure 6.6) and no histogram is shown for this concentration. The decreasing hillock height is most likely the result of the increased cluster concentration and size at the sample surface. Furthermore, the increased copper concentration at the films top layer causes a higher $\mathrm{sp}^{2}$ hybridization decreasing in turn the hillock height. As seen in figure 6.6(a), at concentrations of $5 \% \mathrm{Cu}$, no hillocks could be observed being in accordance with the development seen up to $2 \%$ in the histograms. The average hillock height at low $\mathrm{Cu}$ concentrations is larger than the value measured for the pure ta-C film (compare figure 6.3). The data is not directly comparable because of different experimental conditions.

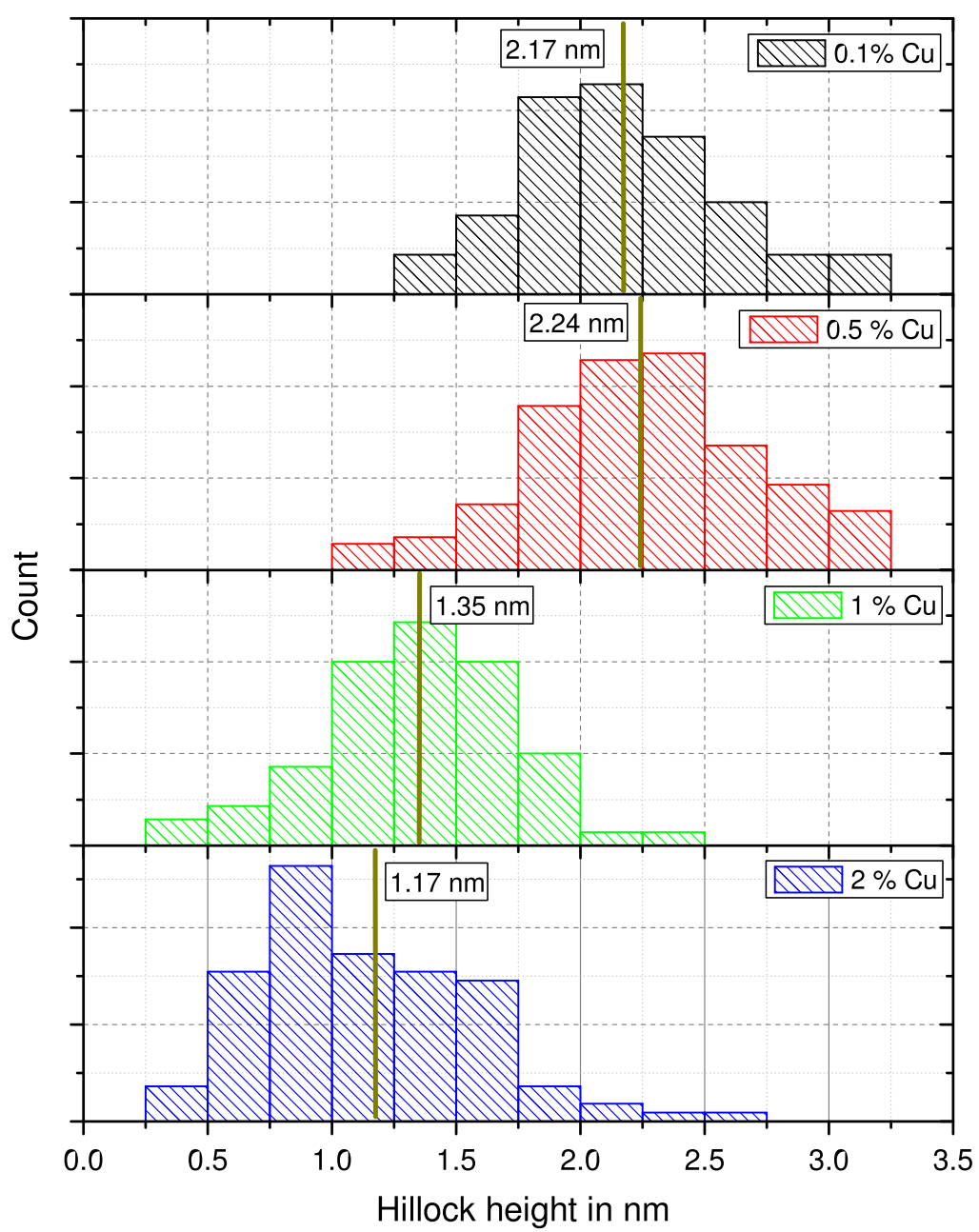

Figure 6.7: Histograms of the hillock height distribution of ion tracks in ta-C:Cu with different $\mathrm{Cu}$ concentrations showing Gaussian distributed results with a FWHM of about $1 \mathrm{~nm}$. The hillock height decreases at higher copper concentrations noticeably 
Analogously to the hillock height, the conductivity distributions of the tracks are shown in figure 6.8. The distribution for $0.1 \% \mathrm{Cu}$ is comparable to pure ta-C and the arithmetic average is also very similar. At $0.5 \% \mathrm{Cu}$ the average conductivity is significantly increased by a factor of 15 . The distribution width is much smaller if normalized to the average conductivity. In addition, the distribution has no significant portion close to $0 \mathrm{~S} \mathrm{~m}^{-1}$ and therefore looks more Gaussian even if it is a Poisson distribution. At $1 \% \mathrm{Cu}$, the track conductivity seems to decrease compared to $0.5 \% \mathrm{Cu}$. The distributions look very similar with only a shift of about $20 \mathrm{~S} \mathrm{~m}^{-1}$. At $2 \% \mathrm{Cu}$, the average conductivity again increases and the distribution becomes very wide. However, the statistical accuracy is low because only a limited amount of data is available for this sample.

The copper has a significant effect on the conductivity of individual ion tracks. Between $0.1 \%$ and $2 \%$ the increase is in the order of a factor 10 to 20. Because of the higher average values, the distributions look more Gaussian. Compared to pure ta-C the distributions are narrower but still show a significant variation of individual tracks. The copper increases the density of states in general increasing the possibility for better track conductivity and decreasing the likelihood of poor tracks. The increased number of current paths per track caused by copper-enhanced DOS decrease the statistical fluctuation reducing in turn the width of the track conductivity distributions. However, the copper does not seem to lead to a more complete transformation of the track material instead, the increased conductivity is caused by the generally higher density of defects. In addition, the presence of larger copper clusters along the track, as well as a generally increased matrix conductivity, may increase the effective track diameter which would increase the electron transport and reduce statistical fluctuation as well.

The trend of $0.5 \%, 1 \%$, and $2 \%$ seems to be erroneous because the middle concentration exhibits the lowest conductivity. A major problem of the AFM analysis is the limited sample area probed. A large fluctuation of track conductivity has been observed by changing the sample position in AFM in case of ta-C:Cu. The data presented was deduced by measuring about 100 to 200 tracks per sample in an area of some $\mathrm{m}^{2}$. Judging the data, the measured areas are not necessarily representative for the overall film. Typical reasons for local fluctuations of surface properties are surface contamination with dirt and oxidation of copper (age of sample).

In figure 6.9 the results of the AFM study are compared to the data obtained by macroscopic contact-pad measurements at room temperature. In pure ta-C both results correspond very well. In case of the copper samples, the AFM measurement resulted in higher overall values. One reason for an increased track current in AFM measurements could be the electric field distribution. In case of the macroscopic setup, the equipotential lines are perpendicular to the track paths, within a thin horizontal plane of the film the surrounding matrix is approximately at the same potential as the track. In AFM mode, the lines are bent around the track axis because the surrounding matrix is not contacted causing a field gradient into the 


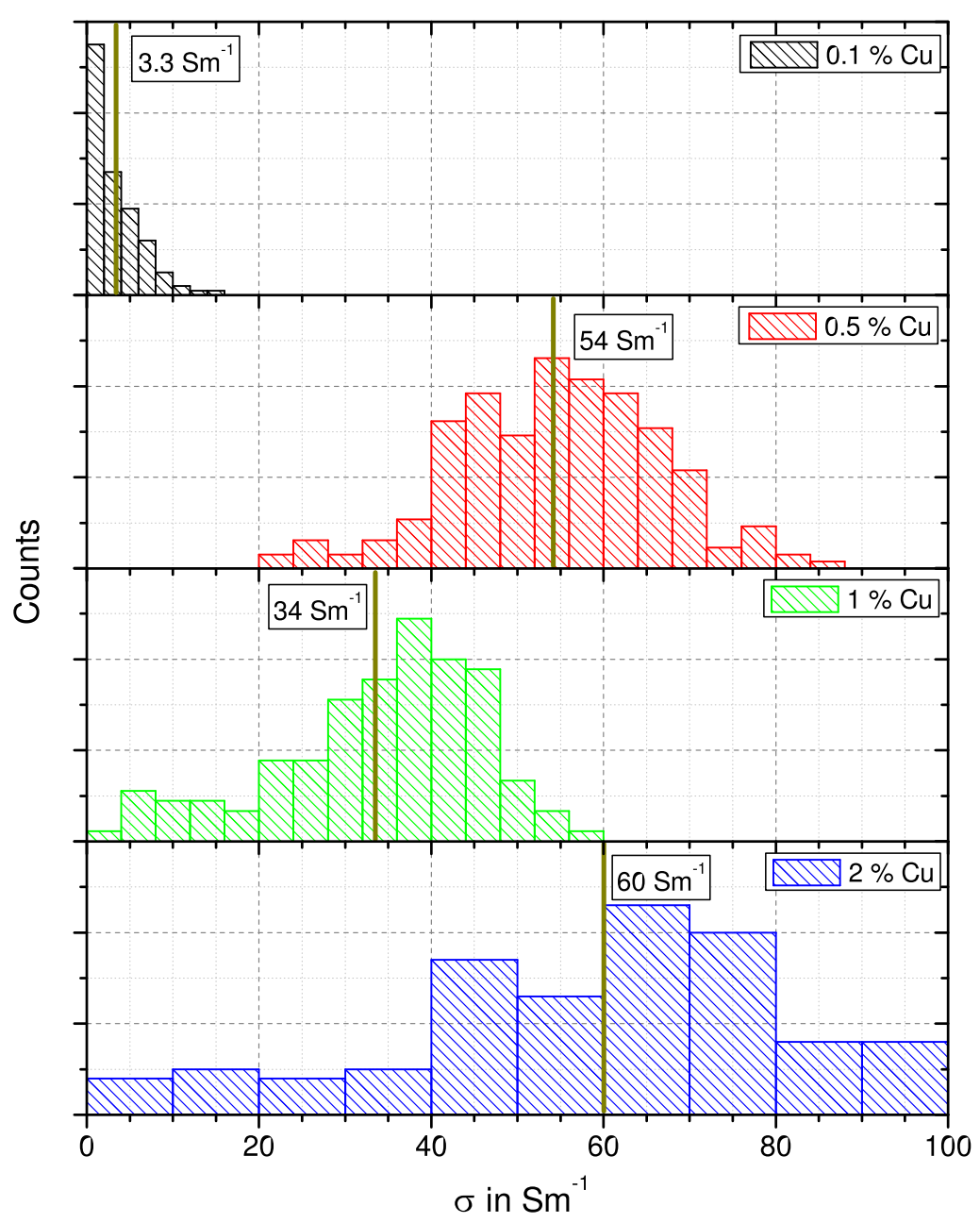

Figure 6.8: Histograms of the track conductivity distributions in different ta-C:Cu films. The smallest copper concentration leads to similar results as observed in pure ta-C. Higher concentrations increase the conductivity significantly. Furthermore, the width of the distributions (normalized to averages) are reduced. The absence of very poor tracks lead to more Gaussian distributions.

surrounding matrix. The macroscopic measurements of the ta-C:Cu matrices have shown a significant increase of the matrix conductivity, which in turn could increase the track current in AFM contact mode. The effect is enhanced by larger Cu clusters at higher $\mathrm{Cu}$ concentrations further increasing the effective track diameter for current transport.

In addition to actually increased track currents in AFM, sources of error and local fluctuations should be considered. The AFM measurements are very sensitive to surface contamination and surface oxidation of the copper of the samples. In some occasions, vast variations in conductivity in AFM measurements on a single ta-C:Cu film have been observed at different locations. The difference of the mean conduc- 
tivity at two locations can be in excess of $50 \%$, far beyond statistical fluctuations. A possible explanation is a locally different level of surface oxidation and contamination. Furthermore, the measured areas are microscopic $\left(1 \mathrm{\mu m}^{2}\right)$, where the copper surface concentration could fluctuate. A high surface concentration of copper enhances the planar conductivity of the surface increasing the effective track diameter in turn. The high resistivity and homogeneity at a microscopic level of pure ta- $\mathrm{C}$ is lost to some extend by the copper incorporation.

While it is not possible to deduce the best $\mathrm{Cu}$ concentration for highest track conductivity, it is obvious that $0.5 \%$ to $2 \% \mathrm{Cu}$ increase the track conductivity significantly. The values seem to be larger than measured in macroscopic measurements. Considering the sources of errors, the data of the macroscopic and microscopic experiments do not contradict each other. While the macroscopic contact pads, for a specific copper concentration, result in similar and reproducible data, the small areas covered by AFM measurements are not as stable for different locations on a single sample containing $\mathrm{Cu}$. On the other hand, each sample position gives stable results in AFM measurements if measured again. In order to produce representative data of ta-C:Cu films, much larger data-sets are required to improve the confidence interval and detect systematic deviations of microscopic areas analyzed.

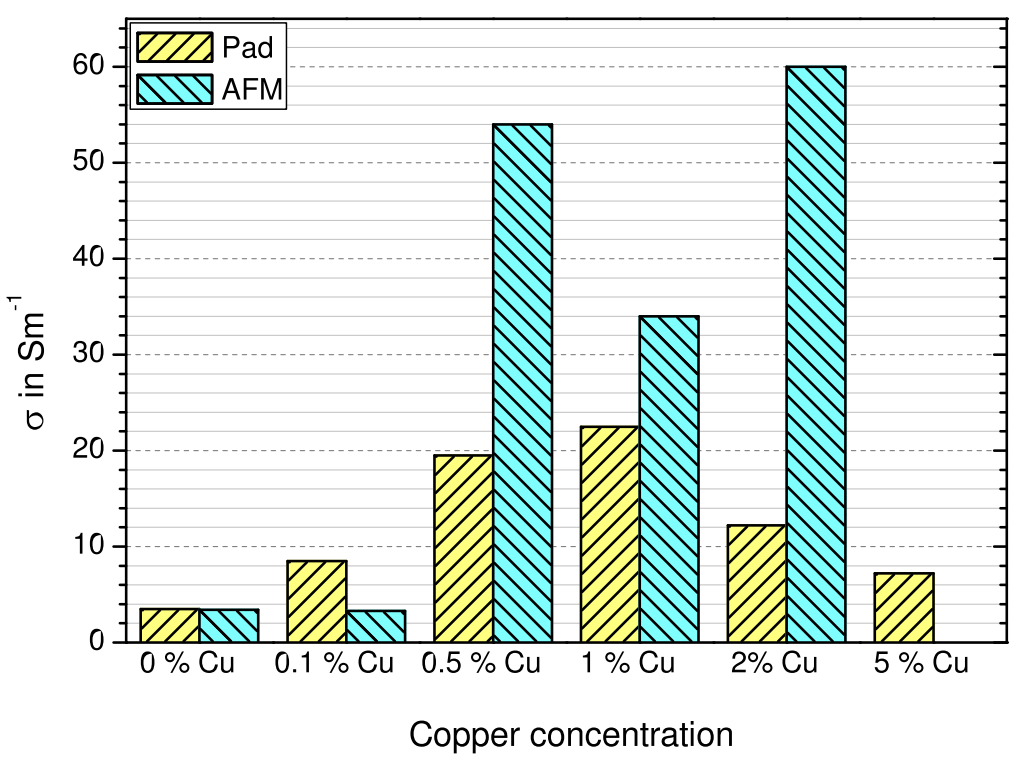

Figure 6.9: Comparison of normalized track conductivities extracted from AFM and macroscopic pad measurements at room temperature. The results for pure ta-C are in very good agreement, while the results for ta- $\mathrm{C}: \mathrm{Cu}$ differ. Both methods result in elevated conductivities of doped ta-C. However, the values extracted from AFM measurements are larger. Most likely explanations are the normalization factors which depend on the exact number of contacted tracks, the large statistical fluctuation of the AFM data, the local (microscopic) fluctuations of the properties of the ta-C:Cu film surfaces as well as the different field geometries of the setups. 


\subsection{Effect of charge state selection}

So far, all samples presented were irradiated by $1 \mathrm{GeV}$ uranium. The ions with originally $2.8 \mathrm{GeV}$ were slowed down to $1 \mathrm{GeV}$ by an aluminum foil placed in front of the ta-C films. This moderation yielded a charge state distribution of the impinging ions centered around the equilibrium charge state. In the following, data of conductive ion tracks formed by irradiations with charge selected $\mathrm{Pb}$ ions is presented. For each sample, all impinging ions had the same charge state upon impacting the ta-C film.

In figure figure 6.10, the topography and corresponding current-maps are shown for the three different irradiation conditions available. The number of track varies and correlates with the irradiation fluence of each sample. Again, each hillock corresponds to an area of high conductivity on the sample. Obviously, the track properties are still distributed randomly, the initial charge state is not the only cause for this fluctuation as expected by the concept of incomplete track formation. The stopping power increases with higher ionization of the ion. Therefore, more pronounced tracks are expected for higher charge states. This correlation can be seen to some extend by comparing the current-maps. A more precise comparison is provided in the following histograms of the hillock heights and track currents.

To achieve a high level of comparability, all samples were analyzed on one day using the same AFM tip. The first sample was rechecked at the end of the session to ensure no significant degradation of the tip. Furthermore, the irradiated samples originated from the same film deposited by MSIBD in ADONIS, which had been divided into four pieces prior to irradiation. Each histogram was composed from multiple AFM images to increase the statistical accuracy. Hereby, the best possible consistency of the data should be achieved to ensure the precision of quantitative comparison. No data of ta- $\mathrm{C}: \mathrm{Cu}$ was considered for this experiment because of the local fluctuations induced by the copper impurities.

First, in figure 6.11 the hillock heights are compared. All distributions are Gaussian and the widths of the distributions are very comparable to the results of uranium irradiation with the tendency to be narrower. The statistical uncertainty and the differences in noise reduction of the topography signal do not allow the confirmation of an effect of the fixed charge state on the width of the distribution. However, the mean values increase with higher charge states as expected by a higher stoppingpower with statistical relevance. Between $53+$ and $60+$ the height increases by about $0.5 \mathrm{~nm}$ which is about $25 \%$ of the total height. The difference in energy loss induced by different charge states is about $20 \%$. Schwen [23] et al. and Krauser et al. [24] suggested linear dependence of hillock height on stopping power. A linear extrapolation towards lower values to a hillock height of zero is interpreted as estimation for the threshold energy required for track formation. Applying this method to the $\mathrm{Pb}$ data results in a threshold value for $S_{\mathrm{e}}$ of $10 \mathrm{keV} \mathrm{nm}^{-1}$ to $15 \mathrm{keV} \mathrm{nm}^{-1}$. The expected margin of error is very high because of the small range of stopping powers available for this extrapolation. 


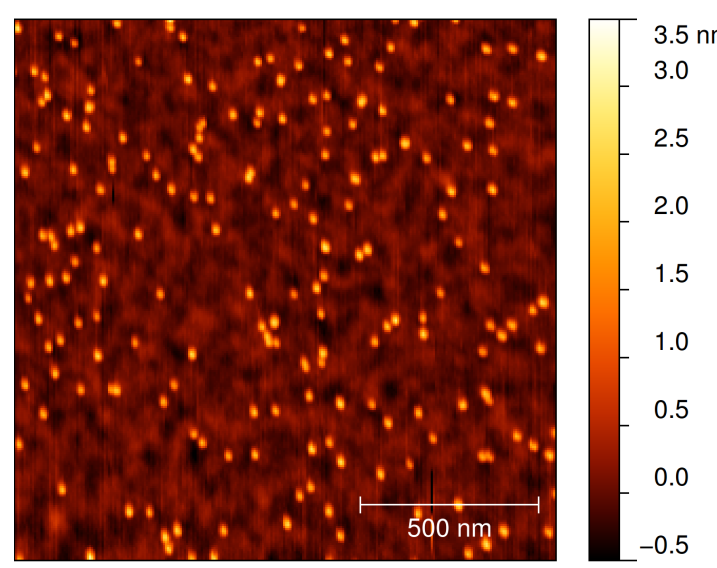

(a) Topography $\mathrm{Pb} 53+$

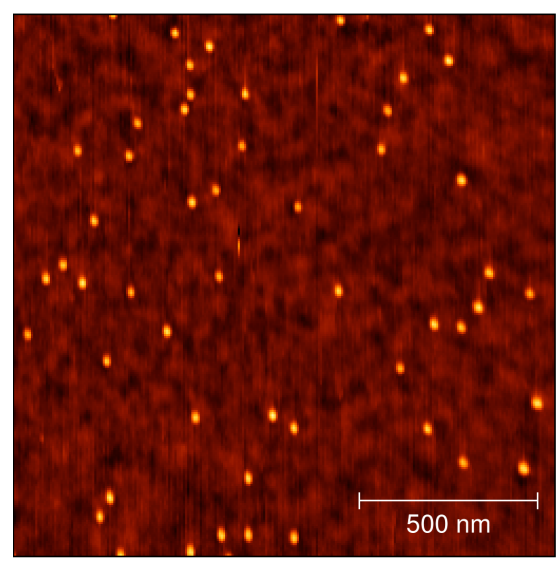

(c) Topography $\mathrm{Pb} 56+$

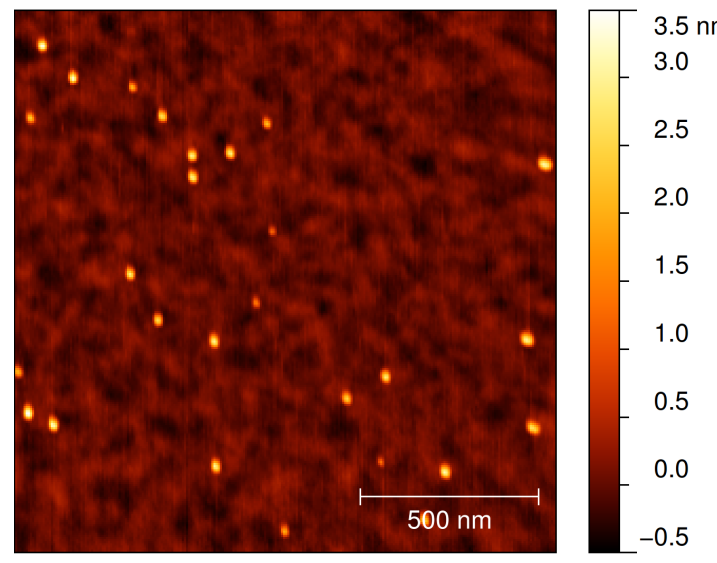

(e) Topography $\mathrm{Pb} 60+$

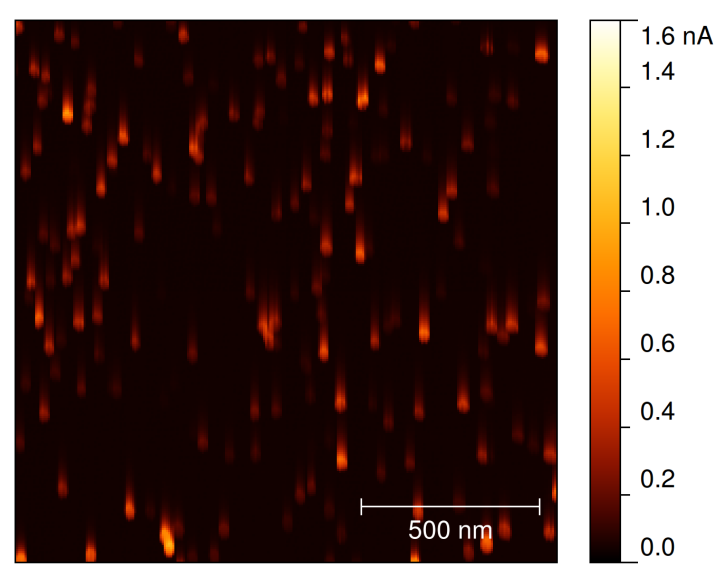

(b) current-map $\mathrm{Pb} 53+$

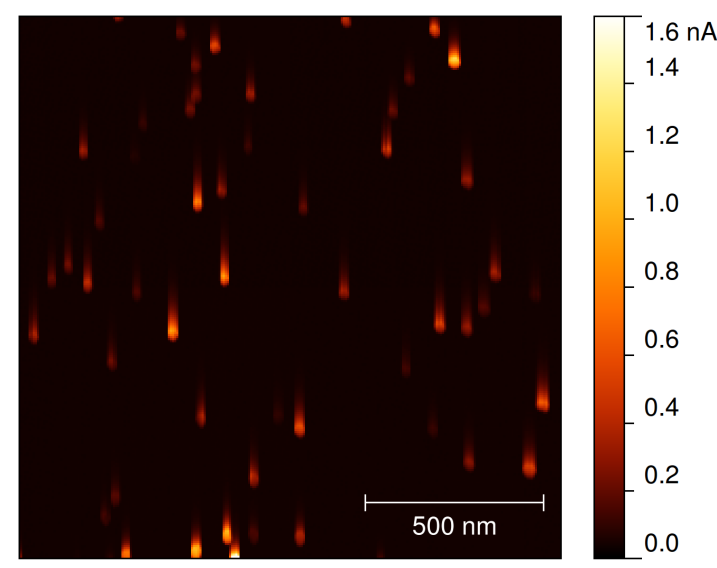

(d) current-map $\mathrm{Pb} 56+$

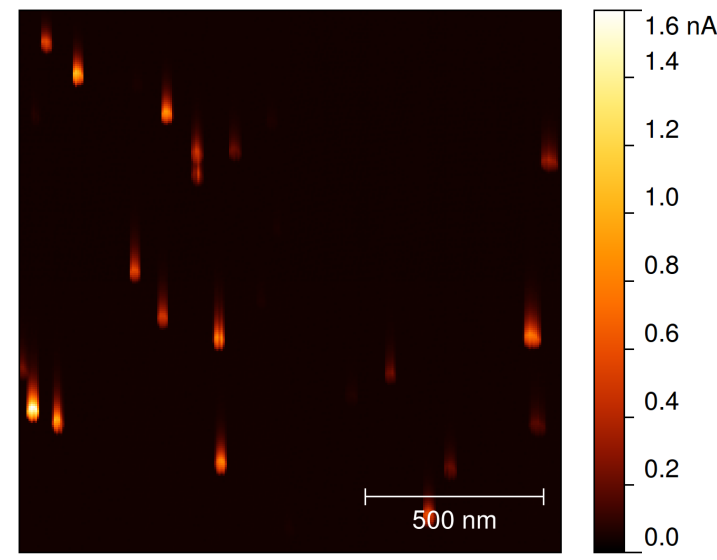

(f) current-map $\mathrm{Pb} 60+$

Figure 6.10: AFM scans showing the topography of hillocks and the corresponding current-map $(0.3 \mathrm{~V})$ of the irradiations of ta-C with different charge states $\left(\mathrm{Pb} 4.57 \mathrm{MeV} \mathrm{u}^{-1}\right)$. The hillock height and current still show a distribution. A slight increase of both hillock height and current with increasing charge state is observed. 


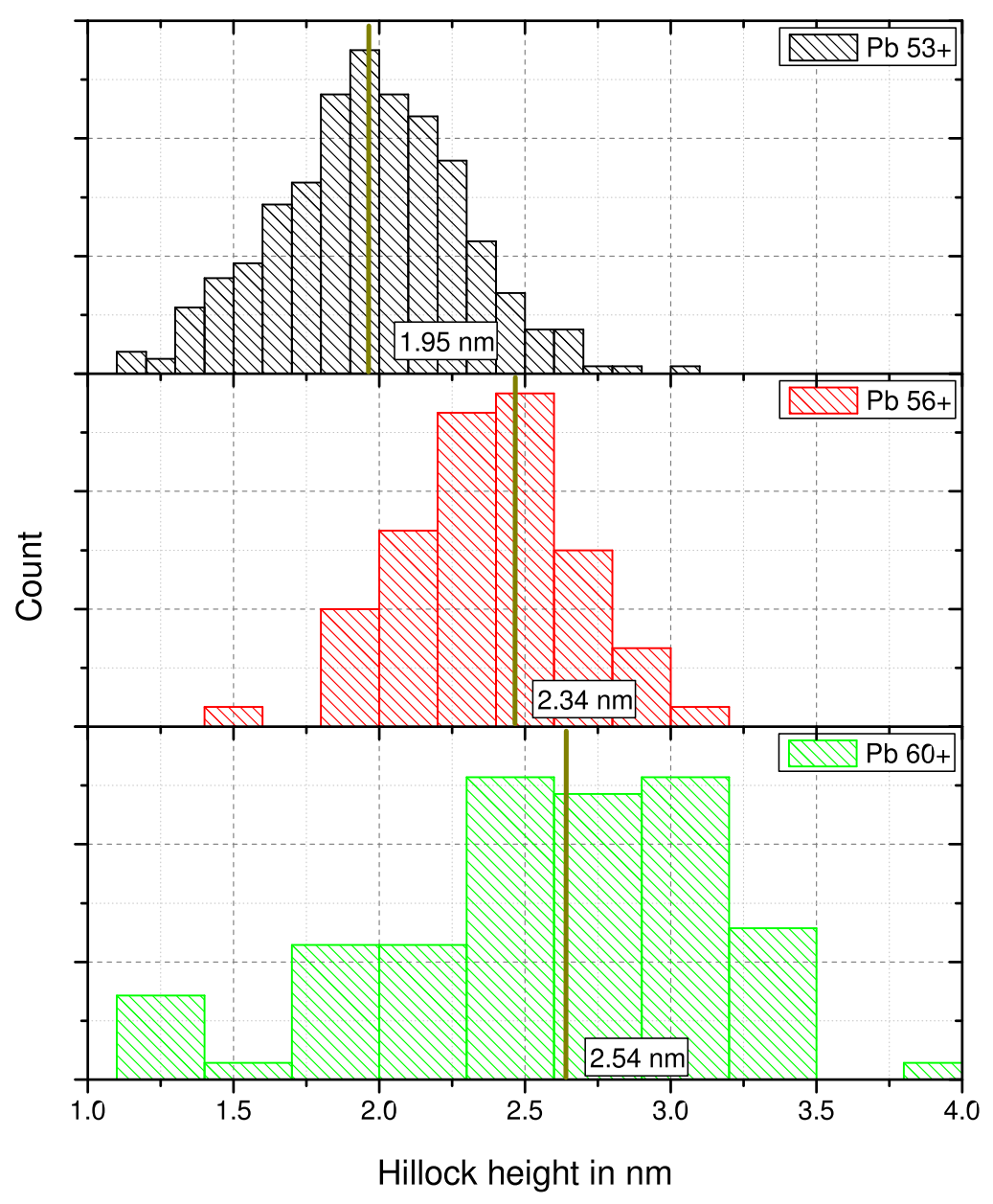

Figure 6.11: Histogram of hillock height distributions of the ion tracks formed by differently charged $\mathrm{Pb}$ ions $\left(4.57 \mathrm{MeVu}^{-1}\right)$. The heights are Gaussian distributed and the averages increase with higher charge state.

The track conductivities are analyzed in the same manner and displayed in figure 6.12 including the results of the $\mathrm{U}$ irradiation in comparison. Again, the distributions are Poisson-like for all samples similar to the results obtained with U-ions. The statistical precision of the $60+$ irradiation is not as good resulting in a higher fluctuation of the data. The averages increase with higher charge state and stopping power. The energy loss of $\mathrm{Pb}^{60+}$ ions is comparable to the average energy loss of $\mathrm{U}$ ions with the equilibrium charge state distribution (simulated with CasP). The extracted conductivities are corresponding reasonably well considering that $\mathrm{Pb}$ and U irradiated samples were analyzed at different bias voltages and with different AFM tips. The widths of the distributions are again mainly governed by the randomness of the track formation process itself. Extrapolating a linear dependency of the average conductivity of the $\mathrm{Pb}$ and $\mathrm{U}$ data as well as the average conductivity using $1 \mathrm{GeV}$ 
$\mathrm{Au}$ ions (charge state distribution resulting in $S_{\mathrm{e}}=39 \mathrm{keV} \mathrm{nm}^{-1}$ and $\sigma=0.63 \mathrm{~S} \mathrm{~m}^{-1}$ ) leads to a threshold stopping power of about $36 \mathrm{keV} \mathrm{nm}^{-1}$ which is much larger than the value received by extrapolation the hillock data $\left(10 \mathrm{keV} \mathrm{nm}^{-1}\right.$ to $\left.15 \mathrm{keV} \mathrm{nm}^{-1}\right)$. Krauser et al. [24] obtained about $32 \mathrm{keV} \mathrm{nm}^{-1}$ extrapolating the track current using the stopping powers predicted by SRIM. The SRIM values are slightly lower than predictions of CasP leading to a shift in threshold extrapolation. Waiblinger et al. [80] observed conductive tracks using Xe ions with a stopping power of about $22 \mathrm{keV} \mathrm{nm}^{-1}$, a value clearly below the above mentioned threshold. These tracks exhibit a rather low conductivity but contradict a pure linear relationship of stopping power and conductivity close to the threshold of track formation.

Using different charge states clearly shows an effect of the initial state on track formation. The formation of the hillocks is a surface process which is logically influenced by different stopping powers induced by charge states. The thin films analyzed, are only $80 \mathrm{~nm}$ thick. Along this short passage, ion projectiles do not reach equilibrium charge state (chapter 2.3.4). Therefore, the track conductivity of such thin-film tracks are also influenced by the initial ionization significantly.

The formation of conductive ion tracks in thin ta-C films is not only dependent upon the projectile energy but also upon the charge state. The stripping of ions to high charge states is very important before hitting the carbon films. For example, the ${ }^{238} \mathrm{U}^{28+}$ beam available at GSI would induce a stopping of only about $22 \mathrm{keV} \mathrm{nm}^{-1}$ leading to no significant track formation.

The fluctuation of track properties (hillock height and conductivity) do not just depend on initial charge state. The statistical process of incomplete track formation as suggested by Herre et al. [63] is the main factor for the distributions in properties. A high charge state increases the stopping power which leads to enhanced track conductivity. The highest-charged- $\mathrm{Pb}$ irradiations resulted in track conductivities very close to those obtained with equilibrium-charge-state $\mathrm{U}$ ions at the Bragg peak. Highly-charged $U$ is expected to lead to an additional increase in track conductivity. Therefore, the charge state can be used to enhance the stopping power of single ion projectiles. 


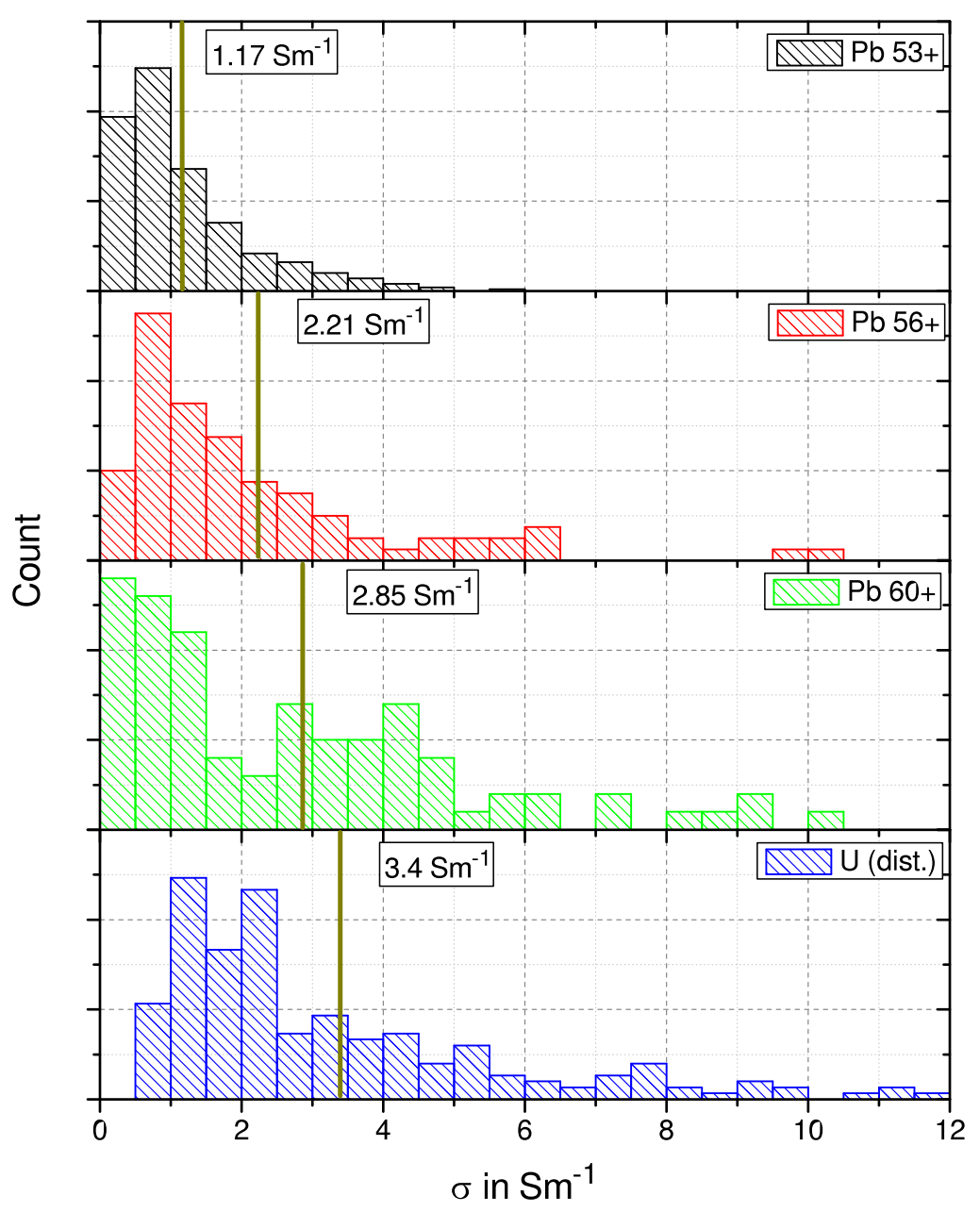

Figure 6.12: Histograms of conductivity distributions of the $\mathrm{Pb}$ irradiation $\left(4.57 \mathrm{MeV} \mathrm{u}^{-1}\right)$ measured by AFM in comparison to $1 \mathrm{GeV}$ U (charge distribution). The conductivity increases with higher charge states each distribution is a rather wide Poisson distribution. 


\subsection{Comparison of temperature-dependent AFM and pad conductivities}

In this section temperature-dependent AFM measurements are presented and compared to the macroscopic pad results. The temperature range extends from room temperature to $100^{\circ} \mathrm{C}$. At different temperatures, current-maps were recorded to produce statistical averages for track conductivities.

Figure 6.13 displays data of the $0.1 \% \mathrm{Cu}$ sample irradiated with $1 \mathrm{GeV} \mathrm{U}$ ions with a fluence of about $2 \times 10^{9} \mathrm{~cm}^{-2}$. The currents, measured by AFM at different bias voltages, are compared to the macroscopic current normalized by the number of tracks under the contact pad. The data shows an increasing conductivity with increasing field and temperature as expected. The error bars were derived from statistical fluctuations of the data. As seen in the plot, the macroscopic data is larger than the AFM data for this $0.1 \% \mathrm{Cu}$ sample, contrary to what is found usually in other samples with higher $\mathrm{Cu}$ content. The $0.1 \% \mathrm{Cu}$ sample had an exceptionally large conductivity in macroscopic measurements whereas the AFM results are more in accordance with the general trends in doping dependence. The reason for the high conductivity of the $0.1 \%$ sample in the macroscopic measurement is unknown. Nonetheless, the difference between both predictions for track conductivities is less than a factor two apart.

In figure 6.14 the currents are converted to conductivities using the linear part of the $I$ - $V$-curves at low bias voltages. The temperature dependence of the AFM data was fitted to thermal activated transport $\left(\sigma=a \exp \left(\frac{-b}{k_{\mathrm{B}} T}\right)\right.$. The resulting temperature dependence $(b=0.17 \mathrm{eV})$ is comparable to the result of the macroscopic measurement plotted in comparison (taken from figure 5.28).

The same experiment was conducted on sample \#1281 with $0.5 \% \mathrm{Cu}$. The results are shown in figure 6.15. Here, the statistical variation of the data points is small and the temperature dependence is well described by the fit with the Arrhenius function. It should be noted for this sample that the AFM data is larger than the pad data (compare figure 6.9). The slope is comparable to the AFM result of the previous sample. Compared to the macroscopic measurements, the slope is noticeable lower. On the other hand, at high temperatures, both measurements approach each other.

The normalized conductivities of both measurements deviate, as already seen in the previous section (chapter 6.2). The temperature-dependent AFM measurements suggest a thermally activated transport process as found before for pad measurements in the respective temperature regime. In case of the sample with $0.1 \% \mathrm{Cu}$, the activation energies (slopes) of both experiments correspond but are lower compared to the results of the macroscopic measurements of the other samples. The AFM characterization of the film with a $\mathrm{Cu}$ concentration of $0.5 \%$ gives an activation energy comparable to the results of the film with $0.1 \% \mathrm{Cu}$ content. However, this value is smaller compared to the pad measurements. Besides the uncertainty caused 
by the small temperature window available in AFM setup, a possible explanation could be the analysis of non-representative areas. In order to gain a conclusive explanation for this deviation of the activation energy, more AFM data and a larger temperature regime would be required.

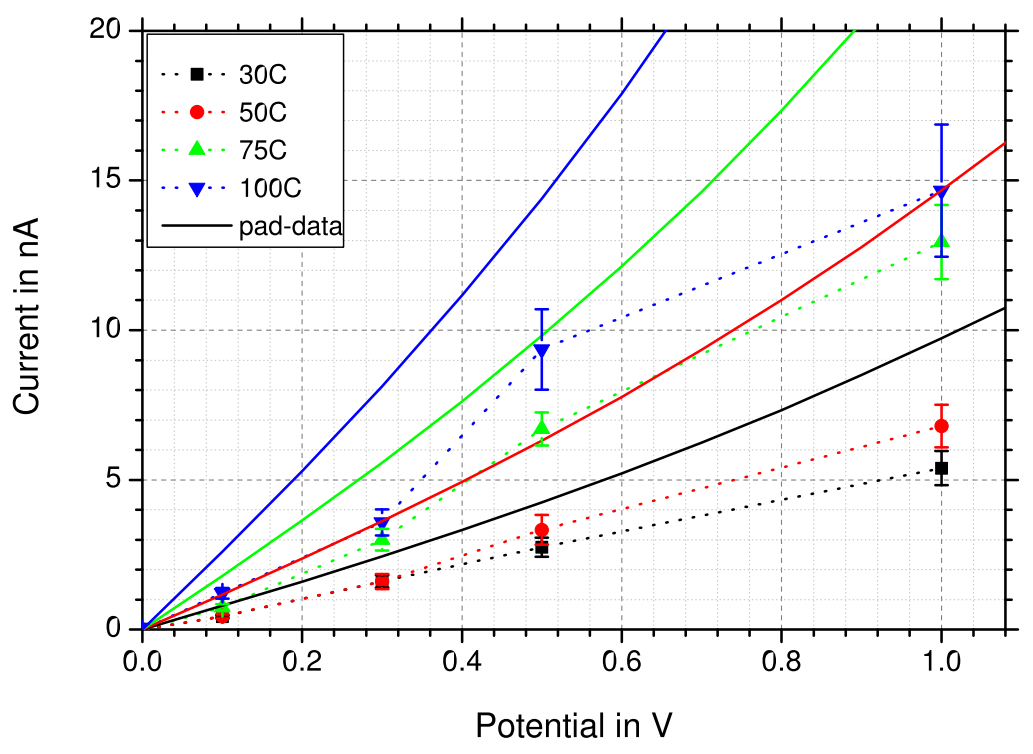

Figure 6.13: Average track current ( $1 \mathrm{GeV} \mathrm{U})$ measured by AFM on ta-C:Cu $(0.1 \% \mathrm{CU})$ depending on temperature and bias voltage in comparison to normalized macroscopic pad data (normalized by the number of tracks under the pad). The data agrees fairly well except for the overall normalization which is off by approximately a factor 1.5. 


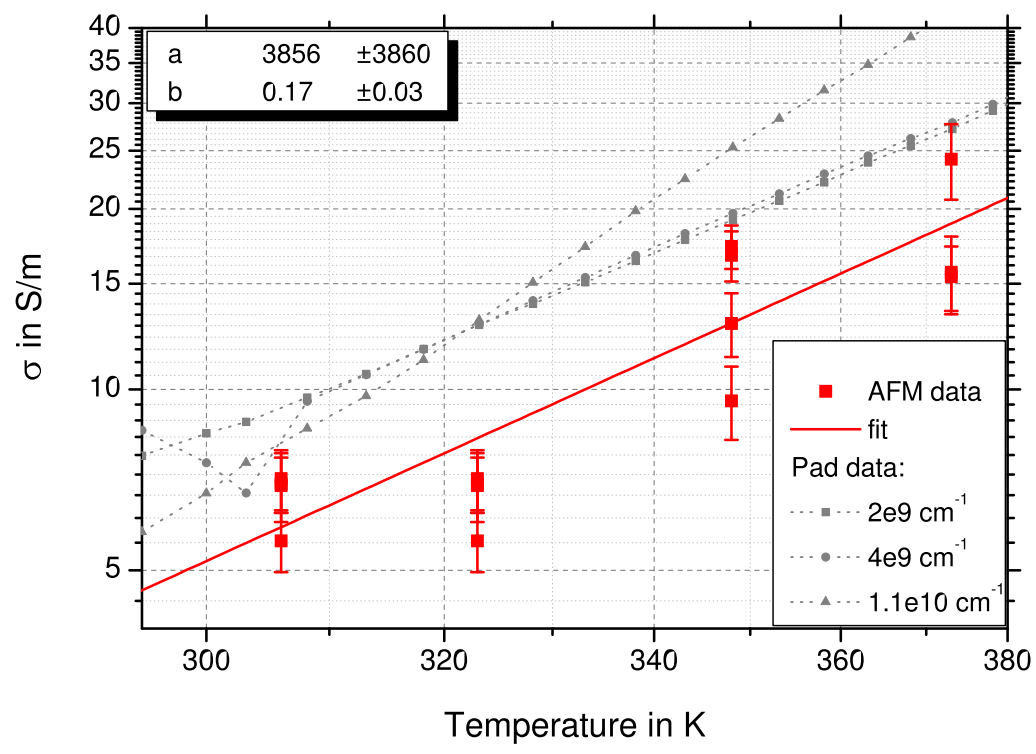

Figure 6.14: Temperature-dependent track conductivity ( $1 \mathrm{GeV} \mathrm{U})$ derived from AFM measurements of ta-C: $\mathrm{Cu}(0.1 \% \mathrm{Cu})$ in comparison with the data taken from macroscopic contact pad measurements (normalized by the number of tracks under the pad). The fluctuation of the AFM data is large, but the average temperature dependence compares well with the macroscopic data. The overall conductivity appears to be about a factor 1.5 smaller.

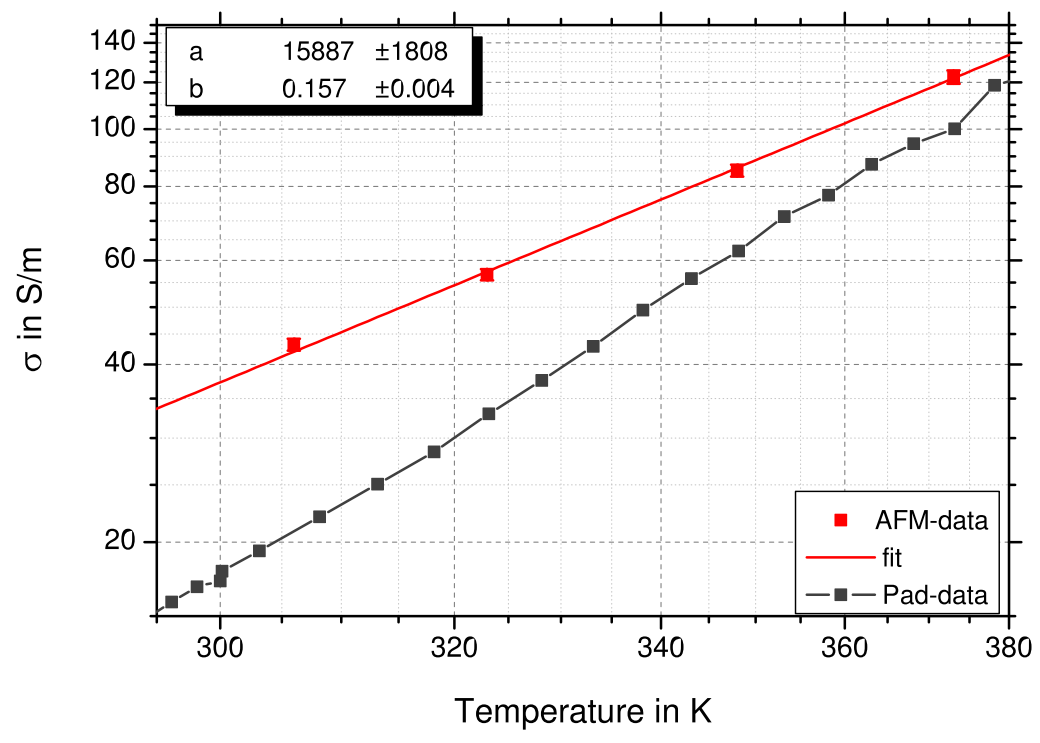

Figure 6.15: Arrhenius plot of the temperature-dependent track conductivity derived from AFM measurements in comparison to macroscopic pad results of ta-C:Cu film with $0.5 \% \mathrm{Cu}(1 \mathrm{GeV} \mathrm{U}$ ions). The statistical errors of the AFM data are small, systematic errors are more likely larger. A thermally-activated transport mechanism describes the data well. The slope of the AFM data $\left(E_{\mathrm{a}}=0.157(4) \mathrm{eV}\right)$ is smaller than the slope of the macroscopic measurement $\left(E_{\mathrm{a}}=0.255(17) \mathrm{eV}\right)$. 


\subsection{Summary AFM measurements}

The AFM analysis has confirmed the statistical fluctuation of track properties (hillock height and conductivity) shown in previous works such as $23,26,33,80]$. The reason for this fluctuation is found in the random transformation from $\mathrm{sp}^{3}$ to $\mathrm{sp}^{2}$ coordination within the track volume as formulated analogously to the findings of Herre et al. [63.

The hillock height follows a Gaussian distribution typical for randomly distributed processes. The conductivity distribution is in most cases Poisson-like as expected for a small number of highly-resistive segments (weak links) determining the transport properties. The number of the dominant resistive sites in a single track is low. Assuming a hopping range of $5 \mathrm{~nm}$ would result in about 16 hopes (weak links) through a track in a typical $80 \mathrm{~nm}$ thick ta-C film. Microscopically, chains of $\mathrm{sp}^{2}$ bonded sides providing good conductivity are randomly interrupted by $\mathrm{sp}^{3}$ configurations (weak links). The density of the $\mathrm{sp}^{3}$ bonds decreases with higher stopping powers. The widths of the conductivity distributions are wide with variations exceeding the average by far. The addition of copper reduced the relative width of the distributions indicating a larger number of hops (shorter hopping range) and more conductive links per track (higher DOS) account for lower overall resistivity.

The normalization of conductivity of the macroscopic pad measurements of large ensembles to a single conductive ion track measured by AFM works well, for pure ta-C. This result indicates the compatibility of the completely different contact geometries formed by the two setups both showing the actual physical behavior of the film. A successful calibration of both characterizations requires high quality contact pads, precise knowledge of the irradiation fluence, and large ensembles of tracks analyzed by AFM providing reliable statistics.

The normalization of ta-C:Cu samples is less precise but still provides similar predictions. The AFM results lead to overall higher track conductivities compared to pad measurements. The fluctuation of conductivities obtained by AFM for different ta-C:Cu samples indicates a large margin of error ${ }^{2}$. The surface accumulation and oxidation of $\mathrm{Cu}$ may lead to local fluctuations of sample conductivity at the surface causing the systematic errors in AFM data. The overall higher conductivity may be caused by the field geometry.

The temperature dependence of the track conductivity was also measured by AFM. Similar to the pad measurements, thermally activated transport was observed above room temperature. The resulting activation energies are smaller. These measurements present a first overview and more AFM data must be recorded to obtain higher statistical reliability. The results of this work indicate the general compatibility of both temperature dependent setups.

On samples with the highest copper concentration of $5 \%$, no signs of tracks were

\footnotetext{
${ }^{2}$ Compare $0.5 \%, 1 \%$ and $2 \%$ Cufigure 6.9
} 
found. The absence of hillocks could be caused by a high copper concentration and $\mathrm{sp}^{2}$ hybridization at the surface. Already at $2 \% \mathrm{Cu}$, the hillocks were significantly reduced in height. Finally, the elevated overall roughness could superimpose small hillocks. The absence of conducting tracks may be the result of missing hillocks leading to a bad contact.

The experiments using charge selected $\mathrm{Pb}$ showed the importance of the initial charge state upon thin film ta-C irradiation. The hillock height and conductivity of tracks increased with higher ionization of the projectile. A high charge state can be used to increase track conductivity and hillock height. However, the distribution of track properties was still present confirming the theory of incomplete track formation as suggested by Herre et al. 63 for stopping powers between the threshold and saturation for track formation [23, 24]. The range until equilibrium charge reached was estimated to be in the order of $10 \mathrm{~nm}$ to $100 \mathrm{~nm}$ 57, 58. Therefore, in case of thin films the initial charge state is of substantial importance. High charge states are suitable to enhance track conductivity.

Overall, a substantial connection can be made between macroscopic and microscopic data concerning the track conductivity. The improved macroscopic setup as well as the sufficient amount of statistics in AFM measurements allows the comparison of both results leading to a much better correspondence compared to earlier attempts 25, 32. 



\section{Characterization of multilayer structures}

Electrically conducting islands of nanometer-sized dimensions (nanodots) combined with nanoleads are essential parts of novel electronic devices based on Coulomb blockade effects 182 184. The low dimensions of these structures allow for single electron effects and the occurrence of quantum effects. A manifestation of the quantum behavior is the Coulomb blockade; the transfer of a single electron onto a conducting nanodot requiring a well-defined amount of Coulomb energy determined by the capacity of the nanodot. An electron-transfer is blocked if this energy is not available [182, 185]. Nanodots and nanoleads are usually constructed by lithographic methods 184, 186. The requirement to reach dimensions of $10 \mathrm{~nm}$ and below poses severe problems and the structures are often rather fragile. Self-organized nanodots are another approach to nano-electronics [185, 187, 188]. Here, the electrical contacts (nanoleads) are the main challenge.

A simple concept to construct nanodots together with the appropriate self-aligned leads in a single step is given by using swift heavy ions impinging an appropriate multilayer target. This principal method, described in figure 7.1. takes advantage of the possibility to modify matter by swift heavy ion irradiation along a single ion track. Ion paths are straight within a few micrometers allowing the fabrication of nano-structures with high aspect ratios. A single ion can penetrate through multiple layers of a film structure transforming material along into a self-aligned ion track. For the device proposed here, the conductive leads and nanodots are formed by ion tracks in ta-C. The insulating layers are made of high resistivity BN, which does not form conductive ion tracks and is easily available in MSIBD.

The properties of the BN synthesized in ADONIS by MSIBD has been studied in detail by Brötzmann [103. He provided an extensive study on the electrical behavior as well as the microscopic structure. The low surface roughness of thin BN films has been confirmed by AFM. Furthermore, neither hillock nor conductive track formation could be observed. All BN allotropes exhibit high resistivities. Because of the technical compatibility and the promising properties of $\mathrm{BN}$, it was used as insulator for the prototype of this device.

Multilayer samples with one or two BN insulator films, embedded in ta-C, were grown and irradiated with $1 \mathrm{GeV}$ Uranium. The top- and bottom-film forming the leads were made of $40 \mathrm{~nm}$ ta-C each. The different thicknesses of the $\mathrm{BN}$ layers and the center ta-C layer are summarized in table 7.1. The formation of interrupted ion tracks upon swift heavy ion irradiation is shown in figure 7.1

Unfortunately, all of these films suffer from slight copper contamination, similar to the sample with $0.1 \% \mathrm{Cu}$ presented above (chapter 5.3). Brötzmann showed an 
increase in conductivity of $\mathrm{BN}$ by a factor of about 30. However, the resistivity of copper contaminated BN still exceeds the value of pure ta-C. Therefore, the results and conclusions drawn from the analysis of these samples should be similar to the expected findings on copper-free samples.

\begin{tabular}{|l|c|c|c|}
\hline name: & $\begin{array}{c}\text { total BN } \\
{[\mathrm{nm}]}\end{array}$ & $\begin{array}{c}\text { first BN } \\
{[\mathrm{nm}]}\end{array}$ & $\begin{array}{c}\text { second BN } \\
{[\mathrm{nm}]}\end{array}$ \\
\hline single BN 6 nm & 6 & 6 & - \\
\hline double BN 12 nm & 12 & 6 & 6 \\
\hline single BN 15 nm & 15 & 15 & - \\
\hline double BN 22 nm & 22 & 6 & 16 \\
\hline
\end{tabular}

Table 7.1: Thicknesses of BN films in multilayers. In case of two BN films, the center ta-C was $8 \mathrm{~nm}$, the leads are made of $40 \mathrm{~nm}$ ta-C (compare figure 7.1).
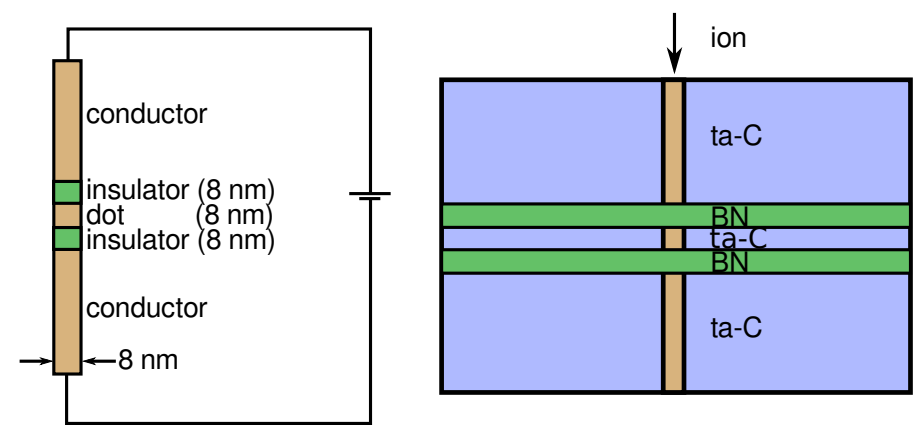

Figure 7.1: Schematics of double barrier structure designed to exhibiting a Coulomb blockage effect (left) as well as conceptional schematics of such double barrier structure realized with an ion track in a ta-C/BN multilayer structure.

The Coulomb blockade is characterized by the Coulomb energy $E_{\mathrm{C}}$, which is required to add an electron to the nanodot:

$$
E_{\mathrm{C}}=\frac{e^{2}}{2 C}
$$

where $e$ is the elementary charge and $C$ the capacity of the nanodot. A higher Coulomb energy and a more pronounced Coulomb blocking effect are achieved for lower capacities. In experiments the $I$ - $V$-characteristics of a nanodot separated by thin insulators between the leads would have a staircase-like appearances [189]. The applied field must reduce the energy levels of the dot by the Coulomb energy, before an additional electron level is activated for current transport. Therefore, the resistance decreases discretely at specific fields applied.

Typically, the Coulomb blockade is observed close to $0 \mathrm{~K}$, where little thermal energy fluctuation is visible. The randomness of the thermal activation would smear 
out the staircase and at sufficiently high temperatures, no signs of Coulomb blocking would be visible [183]. The general requirement for the observation of the effect is $k_{\mathrm{B}} T \ll E_{\mathrm{C}}$. However, very small capacities have led to the observation of the effect at room temperatures (see for example ref. [183, 187, 190]).

The charge energy of an idealized nanodot realized by ion tracks in the proposed structures would have an energy in the order of $0.1 \mathrm{eV}$ to $1 \mathrm{eV}$ depending on BN layer width. This estimation was done assuming a double plate capacitor, a track diameter of $8 \mathrm{~nm}, \epsilon_{\mathrm{r}}=7.1$, and an insulator width of $2 \mathrm{~nm}$ to $20 \mathrm{~nm}$. These values would be promising large suggesting a visibility of Coulomb blocking at room temperature.

\subsection{TEM characterization}

The multilayer systems were analyzed by TEM. In a first test, the ta-C was interrupted using two $\mathrm{CN}_{\mathrm{x}}$ layers. Both ta-C and $\mathrm{CN}$ form amorphous films as seen in figure 7.2 displaying a cross section view of this multilayer sample. The central layers of the film are about $8 \mathrm{~nm}$ thick and the interfaces are flat on the nanometer scale. All ions were deposited with an energy of $100 \mathrm{eV}$ using MSIBD. The growth by subimplantation (chapter 2.2) causes an intermixing at the interfaces. The mixing range is in the order of $1 \mathrm{~nm}$ to $2 \mathrm{~nm}$ in accordance with experiments by Neumaier et al. [49]. The TEM image suggests a slight intermixing at the interfaces as well, the width is about $2 \mathrm{~nm}$ being in accordance to the findings of Neumaier et al. and SRIM simulations. Simulations by Hofsäss using SDTRIMSP predict a slightly larger intermixed region of $4 \mathrm{~nm}$ using a deposition energy of $100 \mathrm{eV}$. This first experiment confirmed the plausibility of thin multilayer films with smooth interfaces fabricated by MSIBD.

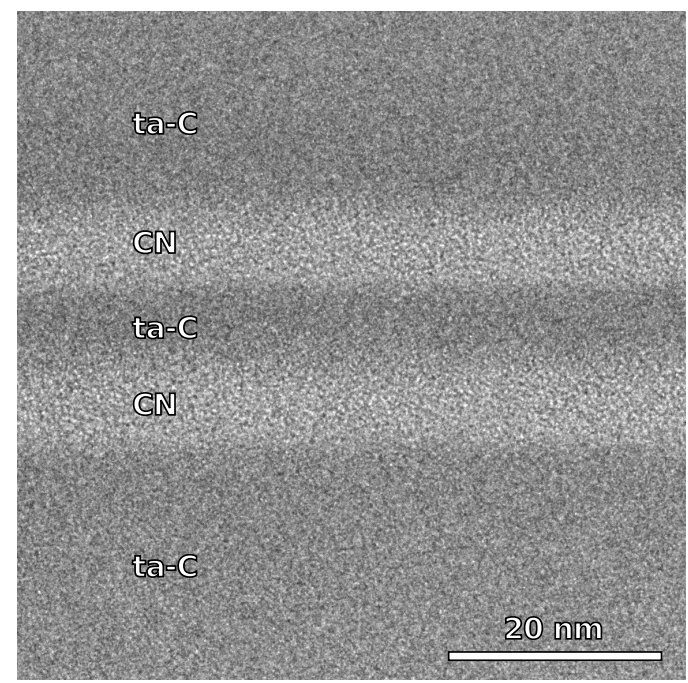

${ }^{1}$ Private communication with H. Hofsäss
Figure 7.2: TEM micrograph of a cross section of a ta-C/CN $\mathrm{CN}_{\mathrm{x}}$ multilayer structure. The ta-C and $\mathrm{CN}_{\mathrm{x}}$ grow amorphously. The interfaces are flat and smooth, a slight intermixing is visible forming $2 \mathrm{~nm}$ interlayers. 
A cross section of a final ta-C/BN sample featuring two insulator layers embedded in three ta-C films is shown in figure 7.3. In the overview (left part of figure) the $\mathrm{Pt}$ glue of the FIB preparation is seen on top of the actual sample. The top and bottom ta-C films are about $40 \mathrm{~nm}$ thick. The BN layers are $6 \mathrm{~nm}$ and $16 \mathrm{~nm}$ separated by a central ta-C film with a thickness of $8 \mathrm{~nm}$. The sputter-cleaning of the silicon prior to deposition leads to an amorphous surface of the substrate. The ta-C grows amorphously on top with a thin intermixed layer. The magnification of the center of the sample (right) reveals the three thin layers. The typical turbostratic structure of the BN is visible (compare to TEM analysis of Brötzmann 103). Even in the very thin top BN film, the structure is formed. Below each BN layer, a thin intermixed ta-C:BN film (1 $\mathrm{nm}$ to $2 \mathrm{~nm}$ width) is visible being in accordance to the results seen in case of $\mathrm{CN}_{\mathrm{x}}$ interlayers. Each ta-C film starts on a mixed $\mathrm{BN}$ : C film as expected by the subplanation model. All film thicknesses, even of thin films, are very homogeneous. The interfaces are smooth and flat on large scales observed in AFM measurements being in accordance with low roughness of ta-C, BN, and multilayer films. The high film quality permits the fabrication of very thin layers on large areas necessary for macroscopic measurements.

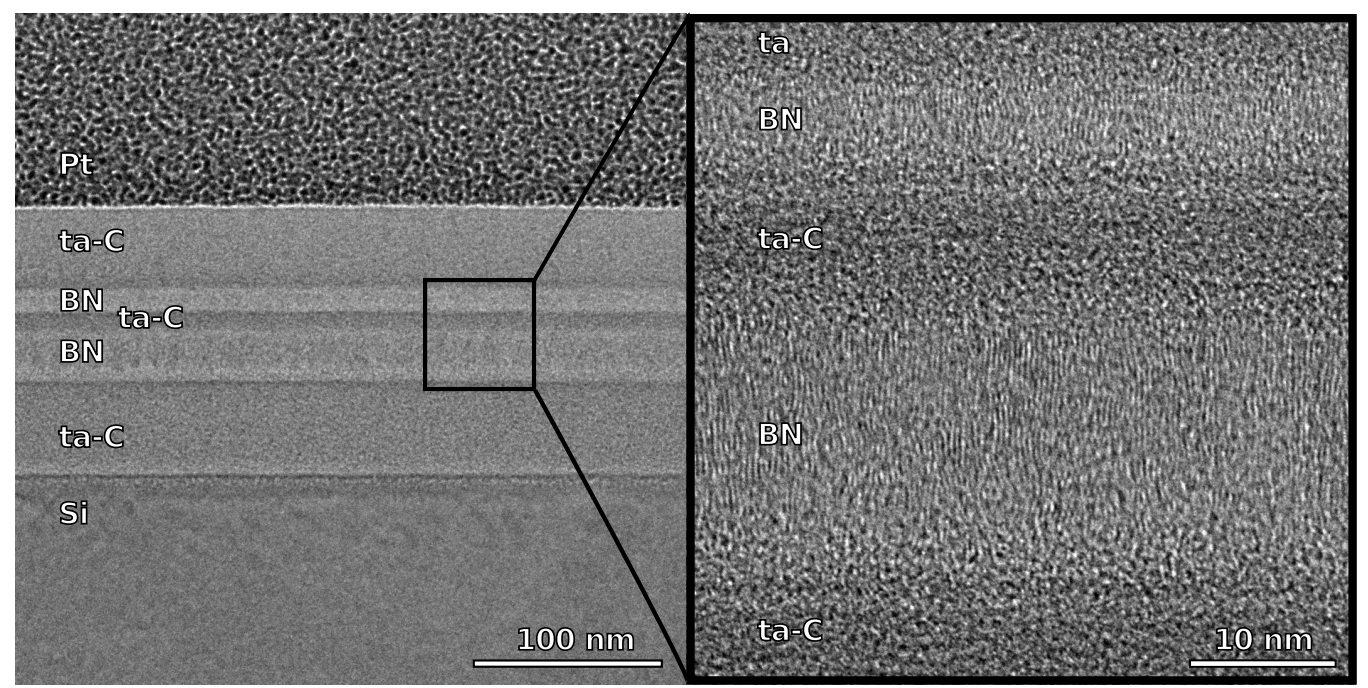

Figure 7.3: TEM micrograph of a cross section view of a ta-C/BN multilayer structure. The ta-C grows amorphously and the BN shows the typical turbostratic structure of BN grown under these conditions in MSIBD 103. The interfaces are flat and smooth, a slight intermixing (about $2 \mathrm{~nm}$ ) is visible at the beginning of each new film. Multilayer structures with very thin layers are possible using ta-C and BN in MSIBD. 


\subsection{Macroscopic electrical characterization}

Analogously to chapter 5, macroscopic contacts were evaporated on top of the multilayer films. The contact area was typically $2.82 \times 10^{-7} \mathrm{~m}^{2}(0.6 \mathrm{~mm}$ pad diameter $)$. Otherwise, the current was normalized to this area. All films are in the order of $100 \mathrm{~nm}$ thick allowing a first order comparison of the currents at specific sample biases. The calculation of specific conductivities has been omitted because of high uncertainties of actual sample geometers and properties (e.g. mixed interfaces) and the true path of the current.

\section{Boron nitride}

A single BN film was grown on top of high conductive n-Si, which has been used for all samples in this work. This film has been contaminated with copper as all multilayer samples. Therefore, it provides a plausible approximation of the BN behavior in the multilayer films. The appearance of the $I$ - $V$-characteristics are comparable to ta-C. In fact, BN can be described as well by the extended FP model in the range of $250 \mathrm{~K}$ to $370 \mathrm{~K} 103$. In figure 7.4 the $I$-T-characteristics of a $93 \mathrm{~nm} \mathrm{BN} \mathrm{sam-}$ ple are plotted. The current was normalized to the contact area of $2.82 \times 10^{-7} \mathrm{~m}^{2}$ from $9.5 \times 10^{-7} \mathrm{~m}^{2}$. The conductivity decreases significantly towards lower temperatures. Between $100 \mathrm{~K}$ to $150 \mathrm{~K}$, a residual conductivity becomes dominant. The conductivity is lower than in case of unirradiated ta-C confirming the good insulation properties of BN. However, the residual conductivity is more pounced becoming dominant at higher temperatures compared to ta-C. Therefore, at lower temperature, the resistivity ratio of $\mathrm{BN}$ to ta- $\mathrm{C}$ decreases, reducing the relative resistivity of $\mathrm{BN}$ at low temperatures and high fields. 


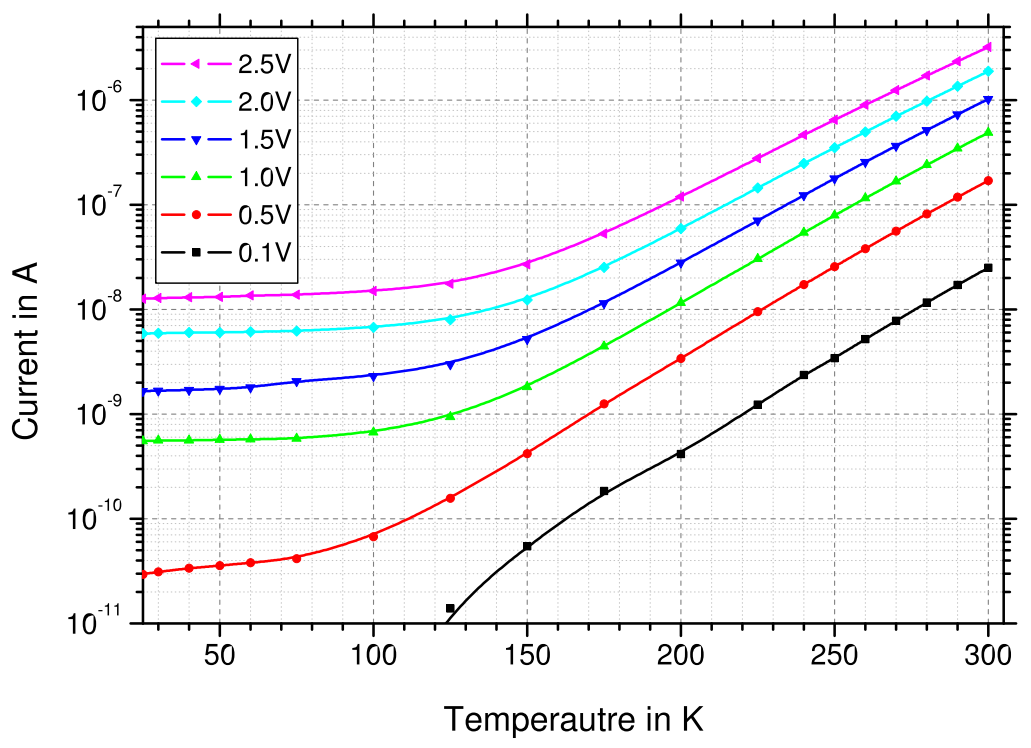

Figure 7.4: $I$-T-characteristics of a $93 \mathrm{~nm} \mathrm{BN}$ film (contact area normalized to $2.82 \times 10^{-7} \mathrm{~m}^{2}$ ). Similar to ta-C the conductivity decreases towards lower temperatures significantly reaching a residual conductivity. The overall resistivity of $\mathrm{BN}$ is larger than the resistivity of ta-C.

\section{Multilayer films}

The multilayer films were characterized electrically in the temperature regime of $20 \mathrm{~K}$ to $300 \mathrm{~K}$ using the same setup and procedure used for ta- $\mathrm{C}$ and ta-C:Cu. The $I$ - $V$-characteristics of both unirradiated and irradiated multilayer structures show an s-shape form at all temperatures. In figure 7.5 some exemplary and representative characteristics are shown for the multilayer sample with two $6 \mathrm{~nm}$ BN insulator films. The two plots show the results at $300 \mathrm{~K}$ and $100 \mathrm{~K}$ for both irradiated and unirradiated films. All characteristics are s-shaped and clearly non-ohmic. At low temperatures, the conductivity decreases significantly. The irradiation increases the conductivity by about an order of magnitude. The sample was irradiated with a fluence of $2 \times 10^{9} \mathrm{~cm}^{-2} \mathrm{U}$ ions. None of the shown characteristics have shown signs of Coulomb blockage. Instead, the characteristics can be simply described by a serial arrangement of $\mathrm{BN}$ and ta-C or tracks.

The qualitative effect of the BN insulator films in macroscopic pad configuration is visible in figure 7.6. All four irradiated multilayer samples, exposed to the same irradiation fluence $\left(2 \times 10^{9} \mathrm{~cm}^{-2} \mathrm{U}\right)$, are shown in comparison to pure irradiated ta-C and BN. The contact pad areas are normalized in order to compare the currents directly. The BN reduces the track conductivity in all cases and thicker overall BN layers cause a higher reduction in current. While the thin BN films reduce the sample conductivity about a factor of 10 to 30, the thickest insulation interlays reduce the 


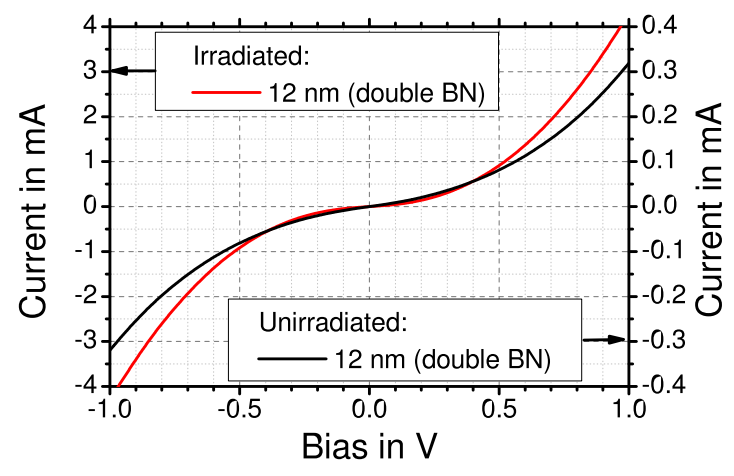

(a) $I$ - $V$-characteristics $(300 \mathrm{~K})$

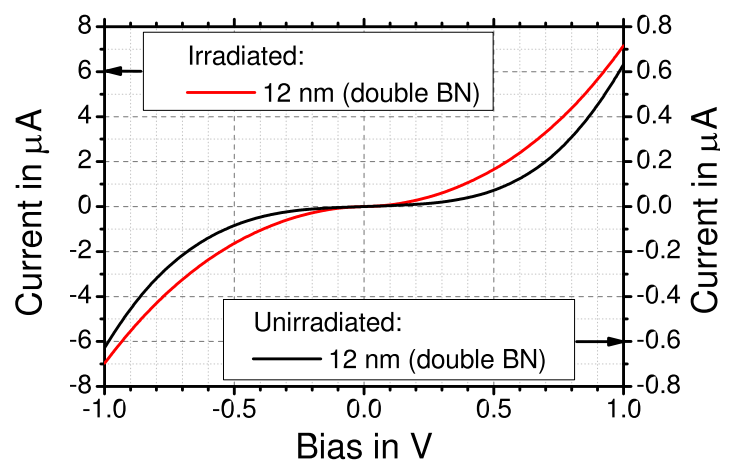

(b) $I$ - $V$-characteristics $(100 \mathrm{~K})$

Figure 7.5: $I$ - $V$-characteristics at $300 \mathrm{~K}$ (a) and $100 \mathrm{~K}$ (b) of a multilayer structure with two $6 \mathrm{~nm}$ BN insulator films. The plots show both the irradiated $\left(2 \times 10^{9} \mathrm{~cm}^{-2} \mathrm{U}\right)$ sample (left scale) and unirradiated case (right scale). The characteristics are representative for the results of all multilayer structures showing the typical s-shaped behavior.

current about three orders of magnitude. The current ratios of the samples are stable between $300 \mathrm{~K}$ and $100 \mathrm{~K}$ caused by similar temperature dependencies of ta-C, tracks, and BN. The single $6 \mathrm{~nm}$ and double $6 \mathrm{~nm}$ interlayer film (12 nm total) show about the same current. A significant difference is only visible at high temperatures and low voltages. Contrary, the thicker insulator films reduce the conductivity dramatically. This observation is explained by the intermixing of the films at the interfaces as seen in TEM analysis. The thicknesses given for the BN layers include such intermixed portions reducing the effective height of each insulator by a few nanometer. The dramatic effect of the interfaces between $\mathrm{BN}$ and ta-C will be more obvious in AFM analysis provided in the next section. 


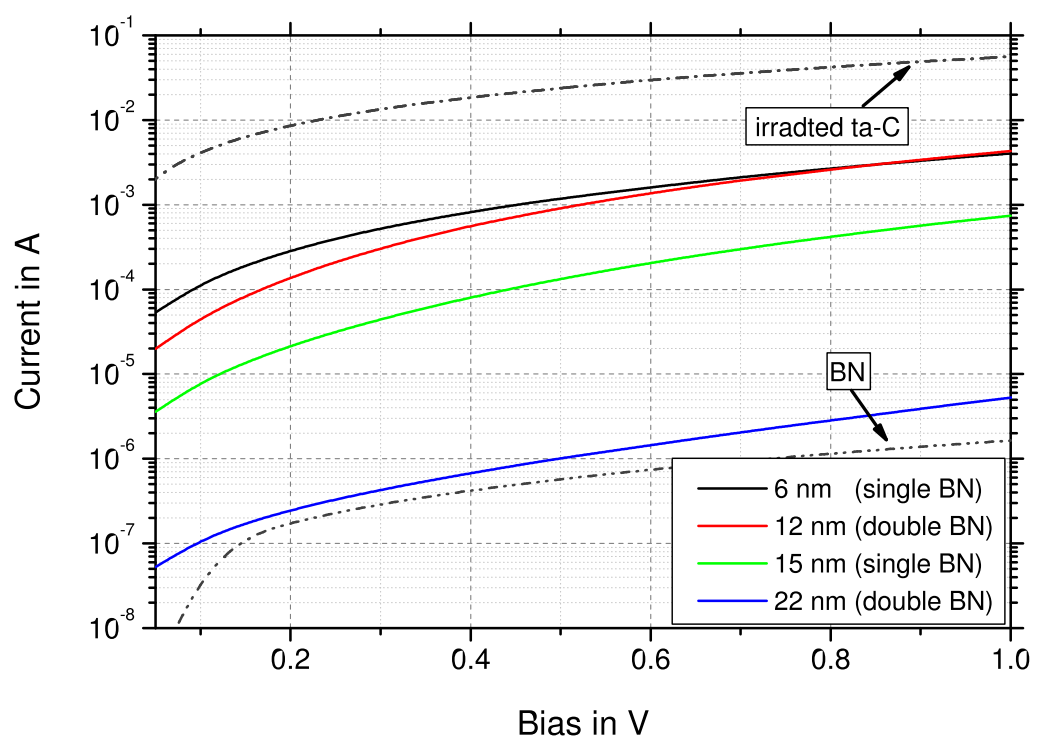

(a) $I$ - $V$-characteristics $(300 \mathrm{~K})$

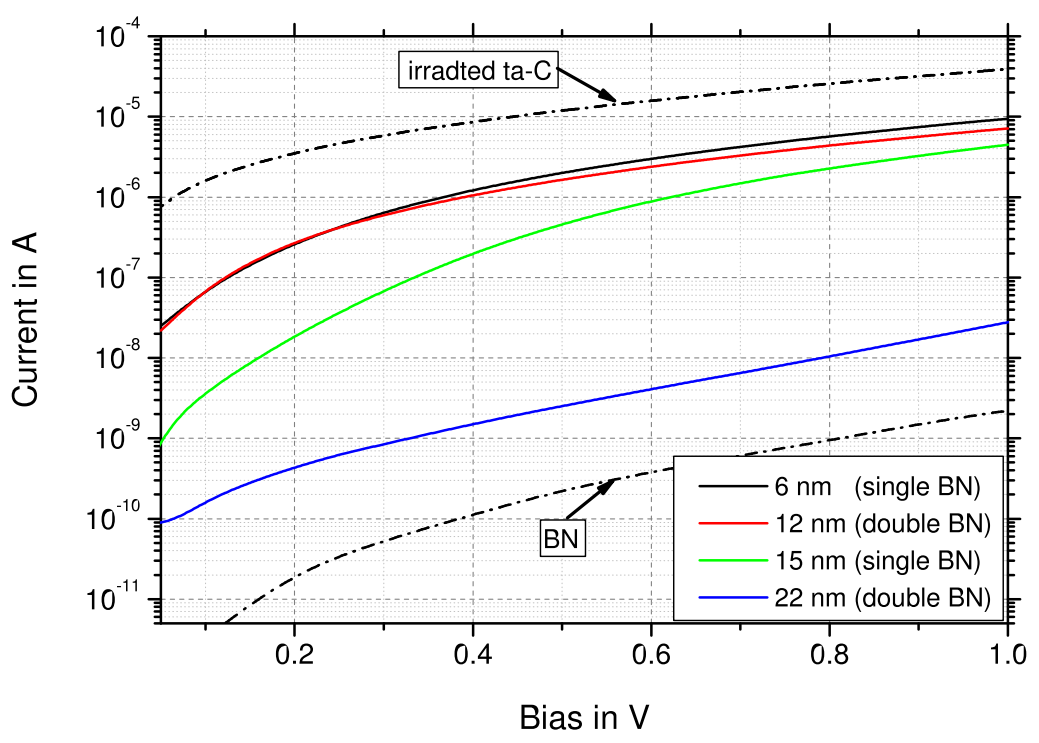

(b) $I$ - $V$-characteristics $(100 \mathrm{~K})$

Figure 7.6: $I$ - $V$-characteristics of all irradiated multilayer films in comparison to continuous tracks in ta-C as well as bulk BN at (a) $300 \mathrm{~K}$ and at (b) $100 \mathrm{~K}$. The insulating effect of the BN is present in irradiated multilayer structures successfully interrupting the ion tracks and increasing the resistance by orders of magnitude. 


\subsection{AFM measurements}

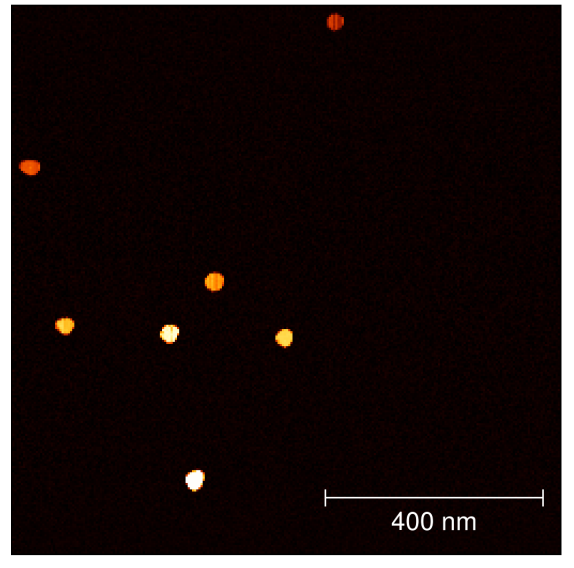

(a) $6 \mathrm{~nm}$ BN single layer

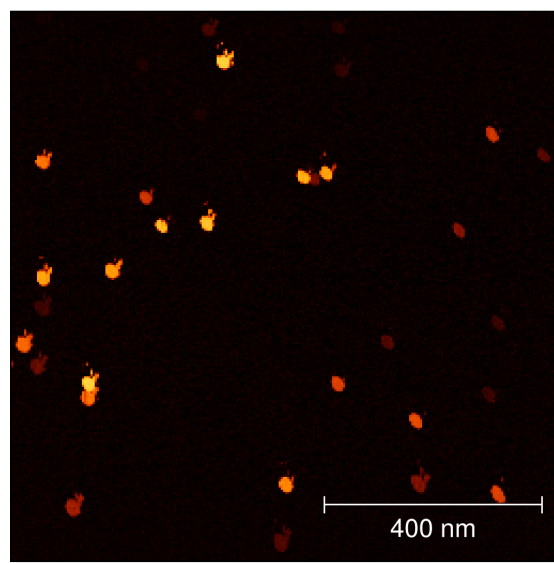

(c) $15 \mathrm{~nm} \mathrm{BN}$ single layer

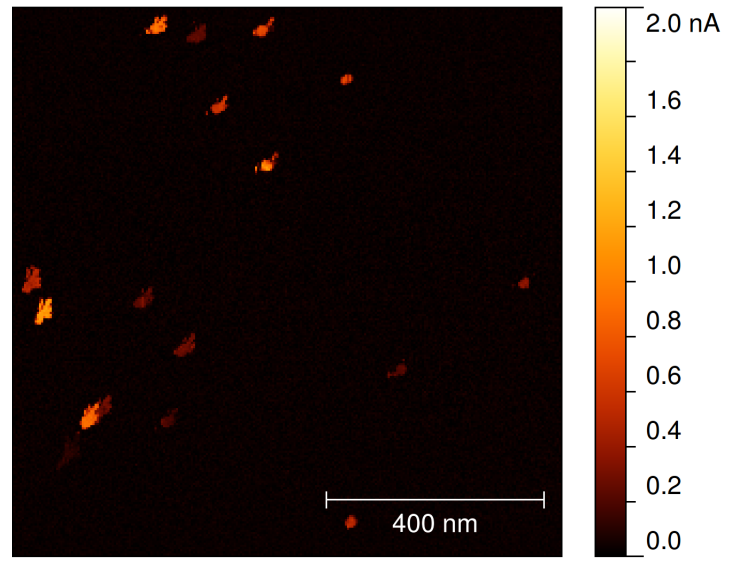

(b) $12 \mathrm{~nm} \mathrm{BN}$ double layer

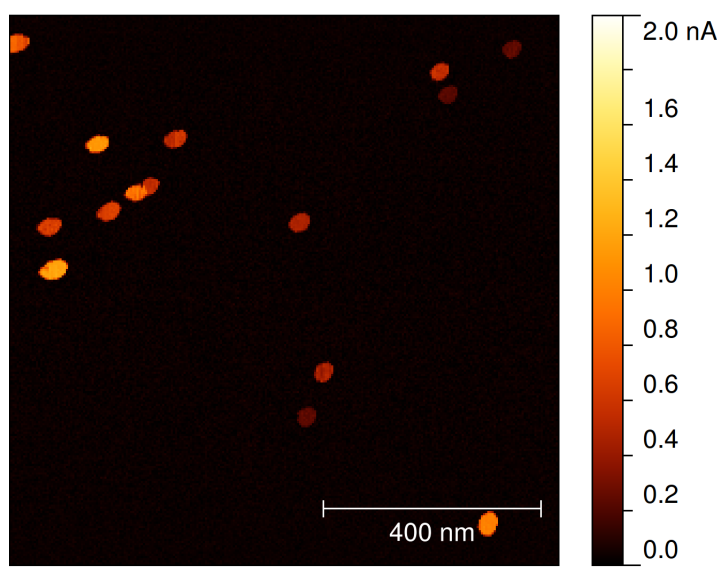

(d) $22 \mathrm{~nm}$ BN double layer

Figure 7.7: AFM Current-maps of irradiated multilayer films with different BN layer configurations. The bias voltage was $0.1 \mathrm{~V}$, all films were irradiated with $1 \mathrm{GeV}$ U ions. The insulating effect of the BN seen in macroscopic measurements seen in figure 7.6 is not visible. The measured track current is hardly affected by the different BN film configurations.

Figure 7.7 shows the current-maps of the irradiated multilayer samples at a bias of $0.1 \mathrm{~V}$. Similar to current-maps of tracks in ta-C, a broad distribution of track currents is visible. Contrary to expectations from macroscopic measurements, the average currents are not reduced as much as expected for increasing BN content of the samples. While macroscopic measurements suggest a factor 1000 between the thin $6 \mathrm{~nm}$ single BN layer sample and the $22 \mathrm{~nm}$ double layer, only a factor of two is seen in AFM measurements. In addition, the currents are generally higher than expected 
from the conductivity of the BN layers and the tracks in ta-C and macroscopic analysis of multilayer samples. An average current of $0.5 \mathrm{nA}$ at $0.1 \mathrm{~V}$ is equivalent to a conductivity of $10 \mathrm{~S} \mathrm{~m}^{-1}$ assuming a cylindrical filament with a diameter of $8 \mathrm{~nm}$. This value is two to three times larger than resulting conductivities for continuous tracks.

The possible explanation for the elevated currents of interrupted tracks in the AFM measurements is found in the interface mixing of the multilayer samples. Boron and Nitrogen increase the conductivity in amorphous carbon by orders of magnitude (compare chapter 2.5.4). The result is a thin, but relatively conductive, film at the interfaces of ta-C and $\mathrm{BN}$ layers. This effect increases the effective contacted area of the ion track at the $\mathrm{BN}$ insulator probably connecting neighboring tracks. In figure 7.8 on the left side, the possible current path through an ion track in a multilayer film is illustrated in case of AFM analysis. Up to the first BN layer, the current has to go through the contacted track. At the interfaces the current can spread through the mixed interlayer reaching neighboring tracks. The BN film is penetrated over a much increased area reducing the resistance effect, annihilating the insulating effect. Beyond the BN layer multiple tracks are contacted further reducing the total resistance measured in AFM mode. The large difference of the normalized currents (factor 1000) obtained in AFM and macroscopic measurements is explained by the short circuit caused by the conductive mixed interlayer.

The right hand side of the schematics shown in figure 7.8 illustrates the reason why this effect is not observed in macroscopic contact mode. Here, the equipotential lines are similar in all neighboring tracks and in the surrounding matrix. It is therefore not possible to connect additional tracks as it is observed in AFM mode. However, the conductive interlayers will still increase the contact area to the BN film. The effect of an increased contact area is illustrated in figure 7.9. The plot shows the macroscopic currents measured on differently irradiated samples with a single $15 \mathrm{~nm}$ BN insulator - The fluence increases by a factor of five while the current is almost constant. The dominating element is the BN insulator, estimating from figure 7.6 at least $90 \%$ of the current drops over this thin film at the lowest fluence. The contact area of the tracks to the $\mathrm{BN}$ does not seem to increase for higher fluences, the complete BN film is already contacted by the conductive mixed interlayer at the lowest fluence available. Again, this observation supports the idea of contacting parallel tracks in AFM setup.

This increase of the contact area makes the observation of the desired Coulomb blocking effect impossible. The mixed interlayer functions as large capacitor plates adding a large capacity to the system which is highly undesired. The effect of B and $\mathrm{N}$ to increase the conductivity of a- $\mathrm{C}$ is an elimination criteria for $\mathrm{BN}$. An additional drawback of $\mathrm{BN}$ is illustrated in figure 7.10 showing the current of a multilayer system in comparison to ta-C. The figure shows the tendency of BN to relatively decrease its insulation property compared to ta-C tracks and matrix towards low temperatures. Both currents of the irradiated and unirradiated multilayer samples 
reduce their resistivity ratio compared to the corresponding ta-C system. Therefore, $\mathrm{BN}$ loses its value as good insulator towards low temperatures.
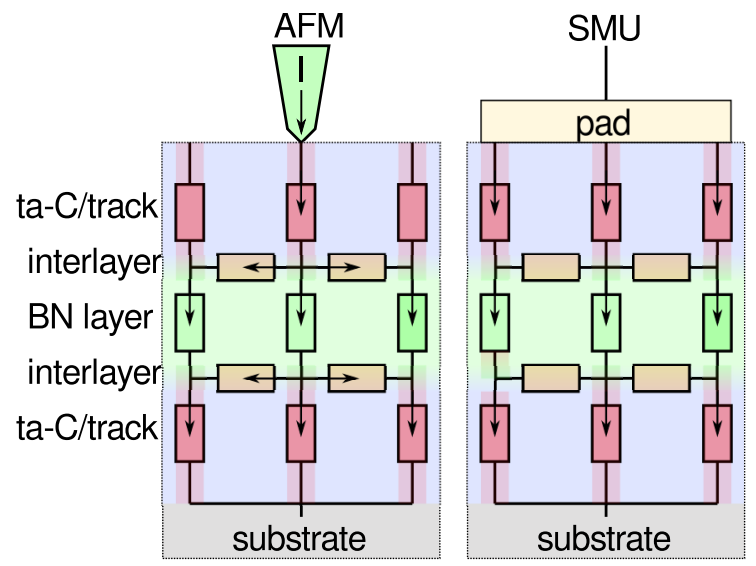

Figure 7.8: Schematics of different conduction paths in AFM and pad measurements. The conductive interlayers between ta-C and $\mathrm{BN}$ will provide planar current paths in AFM mode, neighboring tracks can be connected. In pad mode, all neighboring tracks are already contacted and no additional paths are available. In both cases, the contact area at the insulating $\mathrm{BN}$ is increased reducing the resistivity.

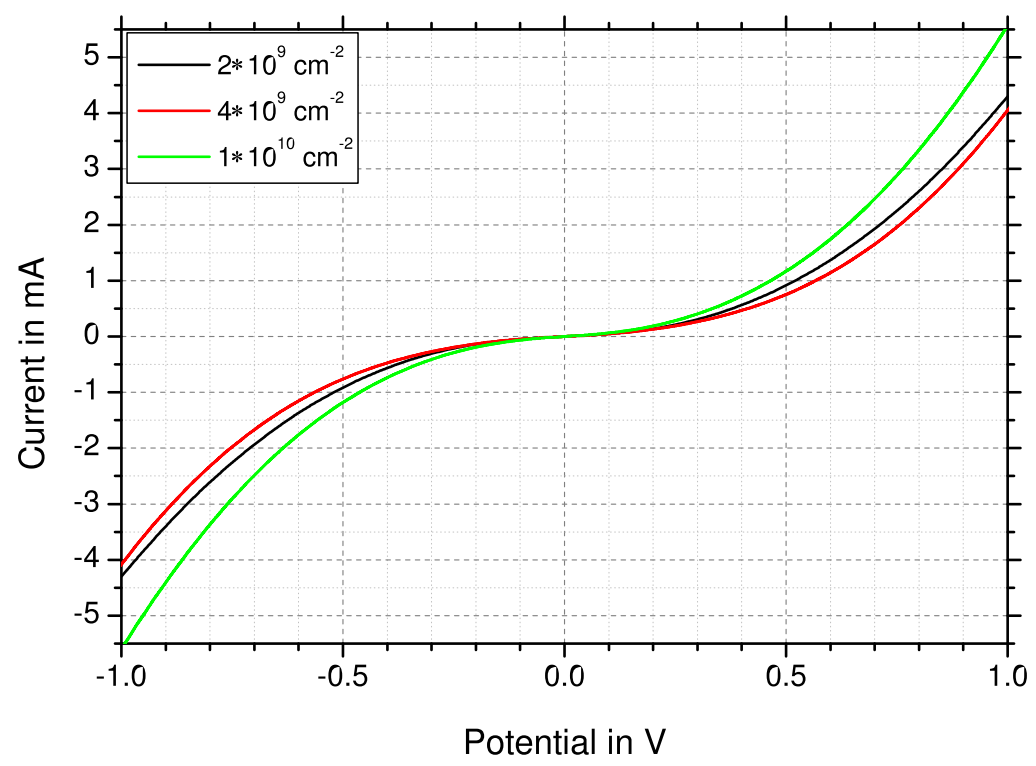

Figure 7.9: $I$ - $V$-characteristics of an irradiated multilayer structure (single $15 \mathrm{~nm} \mathrm{BN}$ ) with different irradiation fluences. The current does not depend much on the number of ion tracks suggesting an increased contact area to the BN film by the conductive mixed interlayers. Even at the lowest fluence the $\mathrm{BN}$ is breached over the total contact area instead of the track area. 


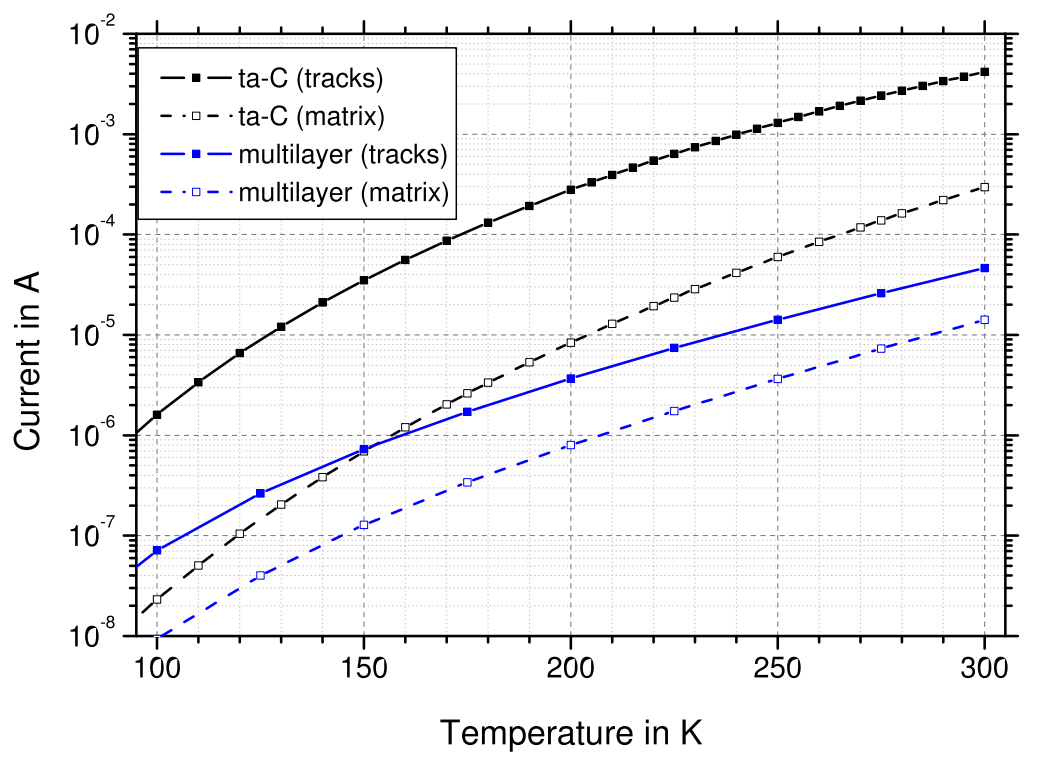

Figure 7.10: $I-T$-characteristics of irradiated and unirradiated ta-C in comparison with a multilayer structure. The insulating effect of the $\mathrm{BN}$ is clearly visible reducing the current significantly. However, towards lower temperature, the ratio ta- $\mathrm{C}$ decreases because of the more pronounced residual conductivity of $\mathrm{BN}$.

\subsection{Summary multilayers}

Although no Colomb blockade could be observed, the fabrication and analysis of these multilayer structures illustrate the possibility to interrupt ion tracks with the aid of thin insulating films. The effect of even $6 \mathrm{~nm} B N$ is significant reducing the track conductivity by one order of magnitude. This effect is macroscopically observed over large areas indicating very homogeneous film properties successfully achieved by MSIBD. The application of a different insulator or the elimination of the conductive mixed interlayers could lead to actually functioning devices.

The mixed interlayers could be reduced or even eliminated by using very low ion energies at the beginning of the growth of of each film. The insulating BN could be replaced by ex-situ fabricated insulators such as thermally evaporated $\mathrm{SiO}_{\mathrm{x}}$ or $\mathrm{SiN}_{\mathrm{x}}$. However, any external step of the delicate growth process of these thin films causes trouble with surface contamination if samples have to be taken out of vacuum. A possible insulator candidate for in-situ deposition by MSIBD is pure Si exhibiting a relatively low conductivity. Unfortunately, it is difficult to provide an N-free $\mathrm{Si}$ beam in the MSIBD setup ADONIS. Therefore, the problems of applying a suitable insulator are caused mainly by technical difficulties but are physically plausible. 


\section{Summary}

\subsection{Conclusions}

The results of the experiments evaluated in this theses confirm and extend the knowledge about electrical conduction processes in ta-C matrix and conductive ion tracks.

The structural analysis by XPS (chapter 4) confirmed the behavior observed by Gerhards et al. [153. Copper tends to accumulate at the film surface and the hybridization ratio starts to move towards more $\mathrm{sp}^{2}$ content at a copper concentration of $5 \%$.

The electrical characterizations (chapter 5) delivered results in agreement with other works on ta-C 25, 27, 103. The most important finding of this thesis is the behavior of the two dominating conduction mechanisms. In principal, they do not change their temperature dependency upon irradiation or the addition of copper impurities. Only the weight of thermally activated as well as tunneling assisted transport shifts and the overall current density increases. In other words, the current transport in ta- $\mathrm{C}$, ta-C: $\mathrm{Cu}$ and the corresponding conductive ion tracks can be explained by the combination of hopping (VRH) and thermally assisted transport (FP) with almost fixed temperature dependencies. The stability of the temperature dependencies, especially of the thermally activated high-temperature mechanism, indicates no change in the DOS of active electron sites. A very similar behavior has already been observed in the unsuccessful quest of doping a-C. The high defect density, as well as the high DOS within the band gap in combination with the chemical behavior of the carbon hybridization, make an introduction of further energy levels hardly possible. Only the density of existing energy levels can be changed leading to different overall current densities. In particular, the conduction is dependent on ratio of $\mathrm{sp}^{2}$ to $\mathrm{sp}^{3}$ hybridization. Each $\mathrm{sp}^{3}$ link will increase the resistivity along a current path. The irradiation directly decreases the density of $\mathrm{sp}^{3}$ links by increasing $\mathrm{sp}^{2}$ hybridization. The addition of copper leads to fine clusters embedded in the ta-C matrix. At the surface of copper, a higher $\mathrm{sp}^{2}$ hybridization of carbon is expected decreasing the number of $\mathrm{sp}^{3}$ links at sufficient copper concentrations. Even at very low concentrations, copper clusters may short circuit high resistivity paths reducing the resistivity as well. Although the addition of copper and the formation of tracks are two different processes, the effect on conductivity is similar. The increased $\mathrm{sp}^{2}$ ratio favors hopping and can be described by variable range hopping. At high temperatures, the thermal activated transport dominates in all films. The reduction of high resistivity links decreases the field dependency; more ohmic behavior is the result in ta-C:Cu films and tracks. Microscopically, the field is highest at points 
of high resistivity. If the number of these points decreases, the local field increases at these locations leading to barrier-saturation (FP barrier is reduced to practically zero) already at low overall fields.

The resulting conductivities of the macroscopic pad characterizations were well described in terms of a simple model. The stretched exponential approach allowed the separate fitting of temperature- and field dependence. This method revealed the observation of the electrical behavior of the ta-C systems described above. In addition, the large temperature range reaching from $20 \mathrm{~K}$ to $380 \mathrm{~K}$ is required to clearly identify residual conductivity, variable range hopping and thermally activated transport. The findings of this thesis extend and connect earlier works by Nix et al. [33] and Schwen 22 proposing variable range hopping for tracks in ta-C as well as the proposed Frenkel-Poole model by Brötzmann [103 for pure ta-C.

The large array of temperature and copper concentration dependent data allowed the determination of a most efficient doping concentration for enhancing track conductivity while keeping a resistive matrix. The maximal enhancement of track conductivity is reached at low $\mathrm{Cu}$ concentrations of $0.5 \%$ to $1 \%$. These tracks are about five times more conductive than their counterparts in pure ta-C. The conductivity of the unirraidated matrix is affected more by copper impurities compared to tracks. Therefore, the conductivity ratio decreases dramatically at high $\mathrm{Cu}$ concentration. However, up to $1 \%$ the increase in matrix conductivity is moderate, making lightly doped tracks interesting for applications. The different effect of copper on matrix- and track conductivity can be understood in terms of the idea of highand low resistivity links. In unirradiated ta-C, the density of high-resistivity links is large. Therefore, copper clusters have a high probability to short circuit some of these points. In tracks, the density is lower decreasing the probability of a copper cluster to bridge a high-resistivity link. In addition, the confined dimensions of a nano-sized track decrease the probability of additional paths. ${ }^{1}$. At very high copper concentration, larger clusters are formed and possibly decrease the rate of material transformation within the track by hindering material transport as well as binding thermal energy required for track formation. This effect of copper is observed by AFM, reducing observed hillock heights of tracks (chapter 6.2).

The results of the AFM characterization of tracks in ta-C are comparable to findings published earlier [24, 28. This work attempted to connect both microscopic and macroscopic electric characterization by normalizing the average track conductivity. In case of pure ta-C, both results matched very well. The comparison of conductivities of different ta-C:Cu showed some deviation, but overall, the normalization delivered similar values. The statistical fluctuation of the AFM data as well as an increasing copper content at the sample surface caused differences in the normalization of a factor of up to four. The temperature dependent AFM data also suggested

${ }^{1}$ Paths are almost only possible along one dimension compared to a possibility of three in case of bulk matrices. 
a thermally activated conduction mechanism. Extracted from these first results the activation energy was lower than predicted by macroscopic measurements. However, the trends led in the same direction. Although, the statistical precision of the AFM data could have been better providing a more solid normalization in case of samples with copper impurities, the results of both characterization methods support each other. The pad measurements have been improved by using $\mathrm{Cr} / \mathrm{Au}$ contact pads as well as applying four-point probing for low resistance samples to reduce contact resistance significantly.

The experiments with selected charge state ions showed the dependency of both hillock height and track conductivity on the initial charge state. An increased charge state led to a higher stopping power and a more complete track formation. Highly charged ions could therefore be used to produce more conductive tracks, when film thicknesses are in the range of $100 \mathrm{~nm}$. The experiment conducted also supports the concept of incomplete track formation in the range of the stopping powers of swift heavy ions. A sole dependency was not observed on initial charge state. The distribution-width of the variation of track properties was hardly changed by chargestate-selection compared to equilibrium-charge-distributed ion irradiations.

Multilayer films were successfully synthesized to produce interrupted conductive ion tracks. Smooth and even interfaces could be produced on large areas. The conductivity of tracks was significantly decreased by the boron nitride insulator layers. However, careful analysis of pad and AFM characterization revealed a thin low-resistivity interface, where $\mathrm{B}$ and $\mathrm{N}$ increase the conductivity of the ta-C. The intermixing was also observed by TEM. These conductive horizontal planes connected neighboring tracks and reduced the insulating effect of the BN. However, the plausibility of the structure is still valid. The conductive interlayers could be reduced by deposition techniques or a different insulator could be tried.

The different experiments have enhanced the understanding of the electronic transport mechanisms of ion tracks in ta-C. Macroscopic- and microscopic characterization have been brought together leading to a more complete understanding. This comparison and the semi-classical model describing the conductivity allow directed interpretation of results obtained from device prototypes. Therefore, this thesis provides additional theoretical understanding of ta- $\mathrm{C}$ and its conductive ion tracks as well as knowledge for practical applications in possible devices. 


\subsection{Outlook}

The results of this work have improved the understanding of the correlation of impurities and tracks in ta-C films. Low copper concentrations of about $1 \%$ lead to an attractive enhancement of track conductivity. In addition, the distribution-width of the randomly varying track-conductivity in ta-C:Cu is reduced especially eliminating tracks, exhibiting high resistivities. Therefore, devices, such as field emission structures and interrupted ion tracks, could benefit by adding small amounts of copper. The matrix conductivity does increase, but within moderate margins. The accumulation of copper at the surface could be reduced by depositing less copper towards the end of film synthesis. A reduced copper concentration at the surface might improve the results obtained in microscopic analysis. In addition, conductivity enhancement of tracks could be achieved by higher charge states. Above-equilibrium-charged uranium is expected to produce even better tracks than highly charged lead.

The multilayer structure could be improved by tuning the insulator. One option is the search for a new material which does not increase the conductivity of ta-C at the interfaces as done by boron nitride. On the other hand, the BN insulator could be improved by decreasing intermixing. First simulations ${ }^{2}$ by Hofsäss demonstrate the plausibility of reducing the intermixing by a factor of two or higher.

The first temperature dependent AFM analysis above room temperature has been promising. Low temperature AFM could be used to further investigate the microscopic behavior of irradiated samples. More hopping dominated transport should be visible at low temperatures. The temperature dependent distribution-widths of randomly varying track-conductivity remain an open field of research as well. The analysis of low- and high-conductivity tracks at different temperatures could further improve the understanding of the variation in track properties.

The conduction in ta- $\mathrm{C}$ has been described by a model of high- and low- resistivity links ( $\mathrm{sp}^{3}$ and $\mathrm{sp}^{2}$ bonds). The idea of such networks could be treated by percolation theory possibly increasing the understanding of the field dependency observed in electrical characterization.

${ }^{2}$ SDTRIMSP simulation using different deposition energies (private communication) 


\section{List of Figures}

2.1 Ternary phase diagram of a-C . . . . . . . . . . . . . . . . . 10

$2.2 \quad$ Stopping power simulations of SRIM and CasP . . . . . . . . . . 15

2.3 Charge state simulation . . . . . . . . . . . . . . 17

2.4 Ion tracks in LiF and energy loss simulation . . . . . . . . . . . . . . 19

2.5 AFM scans of ion tracks in ta-C . . . . . . . . . . . . . . . . . 22

2.6 Schematics of track formation . . . . . . . . . . . . . . . . . 24

2.7 Simplified schematics of band structures _ . . . . . . . . . . . . . . . . 27

2.8 Band schematics proposed by Simmons . . . . . . . . . . . . . . . . 34

2.9 Schematics of the transport mechanisms in localized states . . . . . . 37

2.10 Schematic graph of the band structure of ta-C . . . . . . . . . . 41

2.11 DOS and Inverse participation ratio spectra for different a-C networks 44

3.1 Illustration of the accelerator ADONIS . . . . . . . . . . . . . 46

$3.2 \quad$ XPS spectrum of sputter-cleaned Au target (energy calibration) . . . 50

3.3 Photographs of electrical characterization setup . . . . . . . . . . 53

3.4 Resistance of Silicon substrates used for all samples . . . . . . . . . . 56

4.1 XPS spectra of the C-1s peak and the first plasmon oscillation of ta-C 60

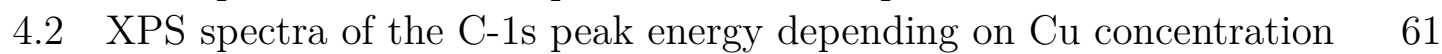

4.3 XPS spectra of the $\mathrm{Cu}-2 \mathrm{p} 3 / 2$ and $\mathrm{Cu}-2 \mathrm{p} 1 / 2$ peaks of $\mathrm{Cu}$ ta-C:Cu . . 62

$5.1 \quad I$-T-characteristics of a virgin ta-C film . . . . . . . . . . . . . 64

$5.2 \quad I-V$-characteristics of a virgin ta-C film . . . . . . . . . . . . . . . 65

$5.3 \quad I-T$-characteristics of a irradiated ta-C film . . . . . . . . . . . . . 66

$5.4 \quad I-V$-characteristics of a irradiated ta-C film . . . . . . . . . . . . 67

$5.5 \quad I-V$-characteristics of a irradiated ta-C film (low temperature) . . . . 68

$5.6 \quad I$-T-characteristics of unirradiated ta-C:Cu with about $0.1 \% \mathrm{Cu}$. . . $\quad 70$

$5.7 \quad I-V$-characteristics of unirradiated ta-C: $\mathrm{Cu}$ with about $0.1 \% \mathrm{Cu}$ content 70

$5.8 \quad I$-T-characteristics of irradiated ta-C: $\mathrm{Cu}$ with about $0.1 \% \mathrm{Cu}$ content 71

$5.9 \quad I-V$-characteristics of irradiated ta-C: $\mathrm{Cu}$ with $0.1 \% \mathrm{Cu}$ content . . . 71

$5.10 \quad I$-T-characteristics of ta-C: $\mathrm{Cu}$ with about $5 \% \mathrm{Cu}$ content $\ldots . . .72$

$5.11 \quad I$-V-characteristics of ta-C: $\mathrm{Cu}(5 \% \mathrm{Cu}) \ldots \ldots . \ldots 73$

$5.12 \quad I$-V-characteristics of irradiated ta-C:Cu $(5 \%) \ldots \ldots \ldots 73$

$\begin{array}{lll}5.13 & I-V \text {-characteristics of unirradiated ta-C:Cu at different temperatures } & 75\end{array}$

$5.14 \quad I$ - $V$-characteristics and extended-FP fits for virgin ta-C $\ldots \ldots 76$

5.15 Correlation of ta-C film thickness $d$ and conductivity . . . . . . . . . 79 
5.16 Zero-bias conductivity of irradiated and unirradiated ta-C:Cu $\ldots . \quad 82$

$5.17 \ln \left(\sigma_{0}(T)\right)$ of ion tracks in pure ta-C with corresponding fit . . . . . 84

5.18 Extrapolated zero-bias conductivity $\sigma_{0}(T)$ of all unirradiated samples 85

5.19 Extrapolated zero-bias conductivity $\sigma_{0}(T)$ of all irradiated samples $\quad . \quad 85$

5.20 Temperature-dependent activation energy for unirradiated ta-C: $\mathrm{Cu} \quad . \quad 90$

5.21 Temperature-dependent activation energy for irradiated ta-C:Cu . . . 90

5.22 Fitting results of the curvature $\beta T^{2}$ of ta-C:Cu matrix and tracks . . 93

5.23 Curvature $\beta T$ for tracks in ta-C:Cu . . . . . . . . . . . . . . 93

5.24 Increase of $\sigma_{0}(T)$ caused by $\mathrm{Cu}$ in unirradiated ta-C:Cu . . . . . . . 98

5.25 Increase of $\sigma_{0}(T)$ caused by copper impurities in ion tracks in ta-C:Cu 98

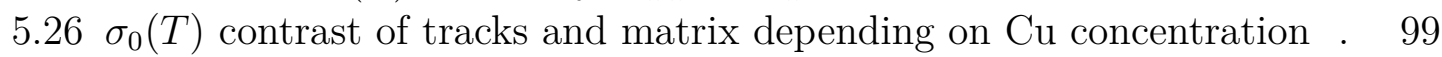

5.27 Current at $300 \mathrm{~K}$ and $0.1 \mathrm{~V}$ for unirradiated and irradiated ta-C . . . 101

5.28 Zero-bias conductivity of ta-C irradiated with different fluences. . . . . 102

5.29 I-T-characteristics of different contact pads on unirradiated ta-C . . . 104

$5.30 \quad I-T$-characteristics of tracks in ta-C deposited on flat n-Si and rougher

$\mathrm{Cr} \ldots \ldots \ldots \ldots \ldots 10 \ldots \ldots \ldots \ldots \ldots$

$5.31 \quad I-T$-characteristics of three measurements on unirradiated ta-C:Cu . . 106

$5.32 I-V$-characteristics of pad measurements on unirradiated ta-C:Cu . . 106

6.1 AFM scans of irradiated ta-C film $(1 \mathrm{GeV} \mathrm{U})$. . . . . . . . . . . . . . 109

6.2 AFM topography of irradiated ta-C recorded with less noise . . . . . 110

6.3 Histograms of track conductivity and hillock height measured by AFM 112

6.4 AFM topography of hillocks on top of irradiated ta-C:Cu . . . . . . . 113

6.5 AFM current-maps of irradiated ta-C:Cu . . . . . . . . . . . . . 114

6.6 AFM scans of irradiated ta-C:Cu film with $5 \% \mathrm{Cu}$. . . . . . . . . 115

6.7 Histograms of the hillock height distribution of ion tracks in ta-C:Cu 116

6.8 Histograms of the track conductivity distributions in different ta-C:Cu 118

6.9 Comparison of normalized track conductivities (AFM) and (macro-

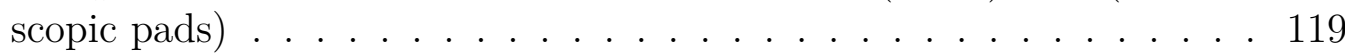

6.10 AFM scans of irradiated ta-C with charge states selected Pb . . . . 121

6.11 Histogram of hillock height distributions formed by differently charged

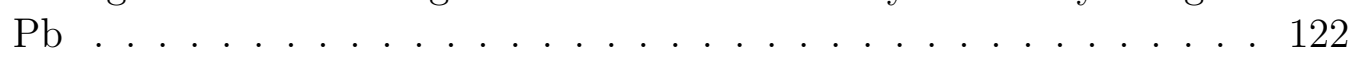

6.12 Histograms of conductivity distributions of tracks formed by singlecharge-state $\mathrm{Pb}$ ions . . . . . . . . . . . . . . . . . . 124

6.13 Temperature-dependent average track current measured by AFM on ta-C:Cu in comparison to normalized macroscopic pad data . . . . . . 126

6.14 Temperature-dependent track conductivity derived from AFM measurements of ta-C:Cu in comparison to data from pad measurements. 127

6.15 Temperature-dependent track conductivity derived from AFM measurements of ta-C:Cu in comparison to data from pad measurements. 127

7.1 Schematics of double barrier structure . . . . . . . . . . . . . . . . . 132 
7.2 TEM image of a cross section of a ta-C/CN $\mathrm{CN}_{\mathrm{x}}$ multilayer structure . . 133

7.3 TEM image of a cross section of a ta-C/BN multilayer structure . . . 134

$7.4 \quad I$-T-characteristics of BN film . . . . . . . . . . . . 136

$\begin{array}{lll}7.5 & I-V \text {-characteristics of a multilayer structure at } 300 \mathrm{~K} \text { and } 100 \mathrm{~K}\end{array}$. . . 137

7.6 $\quad I$ - $V$-characteristics of irradiated multilayer films compared to continuous tracks in ta-C and BN . . . . . . . . . . . . . . 138

7.7 AFM Current-maps of irradiated multilayer films with different BN layer configurations . . . . . . . . . . . . . . . 139

$\begin{array}{lll}7.8 & \text { Schematics of different current paths in AFM and pad measurements } & 141\end{array}$

$7.9 \quad I-V$-characteristics of an irradiated multilayer structure with different irradiation fluences . . . . . . . . . . . . . . . . 141

$7.10 \quad I$-T-characteristics of irradiated and unirradiated ta-C in comparison with a multilayer structure . . . . . . . . . . . . . . . . . 142 


\section{List of Tables}

$2.1 \quad$ List of simulated stopping powers . . . . . . . . . . . . . . . . . . . . 18

5.1 Tabular overview of ta-C samples used for macroscopic electrical char-

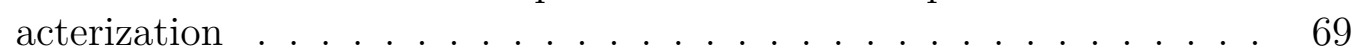

$\begin{array}{lll}5.2 & \text { Fit parameters of the zero-bias fit of } \sigma_{0}(T) \text { for unirradiated ta-C:Cu } . \quad 86\end{array}$

$5.3 \quad$ Fit parameters of the zero-bias fit of $\sigma_{0}(T)$ for irradiated ta-C:Cu.$\quad 86$

7.1 Thicknesses of BN films in multilayers . . . . . . . . . . . . . . . . . 132 


\section{Bibliography}

[1] ATLAS Collaboration. Observation of a new particle in the search for the standard model higgs boson with the ATLAS detector at the LHC. Physics Letters B, 716(1):1 - 29, 2012.

[2] S. Hofmann, V. Ninov, F.P. Heßberger, P. Armbruster, H. Folger, G. Münzenberg, H.J. Schött, A.G. Popeko, A.V. Yeremin, S. Saro, R. Janik, and M. Leino. The new element 112. Zeitschrift für Physik A Hadrons and Nuclei, 354(1):229230, 1996.

[3] Yu. Ts. Oganessian, V. K. Utyonkov, Yu. V. Lobanov, F. Sh. Abdullin, A. N. Polyakov, R. N. Sagaidak, I. V. Shirokovsky, Yu. S. Tsyganov, A. A. Voinov, G. G. Gulbekian, S. L. Bogomolov, B. N. Gikal, A. N. Mezentsev, S. Iliev, V. G. Subbotin, A. M. Sukhov, K. Subotic, V. I. Zagrebaev, G. K. Vostokin, M. G. Itkis, K. J. Moody, J. B. Patin, D. A. Shaughnessy, M. A. Stoyer, N. J. Stoyer, P. A. Wilk, J. M. Kenneally, J. H. Landrum, J. F. Wild, and R. W. Lougheed. Synthesis of the isotopes of elements 118 and 116 in the ${ }^{249} \mathrm{Cf}$ and ${ }^{245} \mathrm{Cm}+{ }^{48} \mathrm{Ca}$ fusion reactions. Phys. Rev. C, 74:044602, Oct 2006.

[4] D. A. YOUNG. Etching of radiation damage in lithium fluoride. Nature, 182(4632):375-377, August 1958.

[5] E. C. H. Silk and R. S. Barnes. Examination of fission fragment tracks with an electron microscope. Philosophical Magazine, 4(44):970-972, 1959.

[6] G. Crozaz, R. Drozd, C. Hohenberg, C. Morgan, C. Ralston, R. Walker, and D. Yuhas. Lunar surface dynamics - Some general conclusions and new results from Apollo 16 and 17. In Lunar and Planetary Science Conference Proceedings, volume 5 of Lunar and Planetary Science Conference Proceedings, pages 24752499, 1974.

[7] R.L. Fleischer and P.B. Price. Techniques for geological dating of minerals by chemical etching of fission fragment tracks. Geochimica et Cosmochimica Acta, 28(10-11):1705 - 1714, 1964.

[8] M. Lang, U.A. Glasmacher, B. Moine, C. Müller, R. Neumann, and G.A. Wagner. Artificial ion tracks in volcanic dark mica simulating natural radiation damage: A scanning force microscopy study. Nuclear Instruments and Methods in Physics Research Section B: Beam Interactions with Materials and Atoms, 191(1-4):346 - 351, 2002. 
[9] C. Trautmann, K. Schwartz, and O. Geiss. Chemical etching of ion tracks in lif crystals. Journal of Applied Physics, 83(7):3560-3564, 1998.

[10] Reimar Spohr. Ion tracks and microtechnology : principles and applications. Vieweg, Braunschweig, 1990.

[11] D.K. Avasthi. Swift Heavy Ions for Materials Engineering and Nanostructuring. Springer Series in Materials Science. Springer London, Limited, 2011.

[12] C. Trautmann, S. Bouffard, and R. Spohr. Etching threshold for ion tracks in polyimide. Nuclear Instruments and Methods in Physics Research Section B: Beam Interactions with Materials and Atoms, 116(1-4):429 - 433, 1996. Radiation Effects in Insulators.

[13] Marek Skupinski, Marcel Toulemonde, Mikael Lindeberg, and Klas Hjort. Ion tracks developed in polyimide resist on si wafers as template for nanowires. Nuclear Instruments and Methods in Physics Research Section B: Beam Interactions with Materials and Atoms, 240(3):681 - 689, 2005.

[14] Florian Maurer, Arti Dangwal, Dmitry Lysenkov, Günter Müller, Maria Eugenia Toimil-Molares, Christina Trautmann, Joachim Brötz, and Hartmut Fuess. Field emission of copper nanowires grown in polymer ion-track membranes. Nuclear Instruments and Methods in Physics Research Section B: Beam Interactions with Materials and Atoms, 245(1):337 - 341, 2006. Proceedings of the Sixth International Symposium on Swift Heavy Ions in Matter (SHIM 2005).

[15] A. F. Bernhardt, R. J. Contolini, A. F. Jankowski, V. Liberman, J. D. Morse, R. G. Musket, R. Barton, J. Macaulay, and C. Spindt. Arrays of field emission cathode structures with sub-300 nm gates. Journal of Vacuum Science 8 Technology B: Microelectronics and Nanometer Structures, 18(3):1212-1215, 2000 .

[16] D. Fink, L.T. Chadderton, K. Hoppe, W.R. Fahrner, A. Chandra, and A. Kiv. Swift-heavy ion track electronics (site). Nuclear Instruments and Methods in Physics Research Section B: Beam Interactions with Materials and Atoms, 261(1-2):727 - 730, 2007. jce:title ¿The Application of Accelerators in Re-

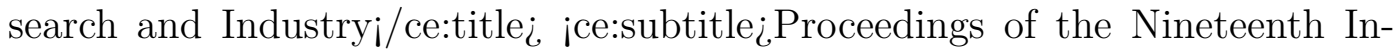
ternational Conference on The Application of Accelerators in Research and Industryi/ce:subtitle ¿ jxocs:full-name¿Nineteenth International Conference on The Application of Accelerators in Research and Industryi/xocs:full-namei.

[17] K. Hoppe, W.R. Fahrner, D. Fink, S. Dhamodoran, A. Petrov, A. Chandra, A. Saad, F. Faupel, V.S.K. Chakravadhanula, and V. Zaporotchenko. An ion track based approach to nano- and micro-electronics. Nuclear Instruments and 
Methods in Physics Research Section B: Beam Interactions with Materials and Atoms, 266(8):1642 - 1646, 2008. Proceedings of the Eighteenth International Conference on Ion Beam Analysis.

[18] H. Walter, W. Prusseit, R. Semerad, H. Kinder, W. Assmann, H. Huber, H. Burkhardt, D. Rainer, and J. A. Sauls. Low-temperature anomaly in the penetration depth of $\mathrm{yba}_{2} \mathrm{Cu}_{3} \mathrm{O}_{7}$ films: Evidence for andreev bound states at surfaces. Phys. Rev. Lett., 80:3598-3601, Apr 1998.

[19] Ch. Houpert, N. Nguyen, F. Studer, D. Groult, and M. Toulemonde. Latent track formation induced by high electronic energy loss in hexaferrites \{AFe12O19\} $(\mathrm{a}=\mathrm{ba}, \mathrm{sr}): \mathrm{A}$ magnetic investigation. Nuclear Instruments and Methods in Physics Research Section B: Beam Interactions with Materials and Atoms, 34(2):228 - 236, 1988.

[20] Giancarlo Rizza, Yaasiin Ramjauny, Marc Hayoun, Sandrine Perruchas, Thierry Gacoin, Patrick Kluth, and Mark C Ridgway. Saturation of the ionhammering effect for large non-hydrostatic capillarity stresses in colloidal silica nanoparticles. Nanotechnology, 22(47):475302, 2011.

[21] Friedrich Aumayr, Stefan Facsko, Ayman S El-Said, Christina Trautmann, and Marika Schleberger. Single ion induced surface nanostructures: a comparison between slow highly charged and swift heavy ions. Journal of Physics: Condensed Matter, 23(39):393001, 2011.

[22] D. Schwen. Structural and electronic properties of swift heavy ion tracks in amorphous carbon. PhD thesis, Georg-August-Universität Göttingen, 2007.

[23] D. Schwen, E. Bringa, J. Krauser, A. Weidinger, C. Trautmann, and H. Hofsass. Nano-hillock formation in diamond-like carbon induced by swift heavy projectiles in the electronic stopping regime: Experiments and atomistic simulations. Applied Physics Letters, 101(11):113115, 2012.

[24] J. Krauser, A-K Nix, H-G Gehrke, H. Hofsäss, C. Trautmann, and A Weidinger. Highly conductive ion tracks in tetrahedral amorphous carbon by irradiation with $30 \mathrm{MeV}$ C 60 projectiles. New Journal of Physics, 13(8):083023, 2011.

[25] A.K. Nix. Swift heavy ion irradiation of semiconducting materials - defect production, phase transformation and annealing. PhD thesis, Georg-AugustUniversität Göttingen, 2010.

[26] J.-H. Zollondz, D. Schwen, A.-K. Nix, C. Trautmann, J. Berthold, J. Krauser, and H. Hofsäss. Conductive nanoscopic ion-tracks in diamond-like-carbon. Materials Science and Engineering: C, 26(5-7):1171 - 1174, 2006. Proceedings of the European Materials Research Society 2005 - Symposium A. 
[27] H.-G. Gehrke. Characterization of swift heavy ion tracks in tetrahedral amorphous carbon and fabrication of self-aligned nano structures. Master's thesis, Georg-August-Univeristät Göttingen, 2008.

[28] J. Krauser, J.-H. Zollondz, A. Weidinger, and C. Trautmann. Conductivity of nanometer-sized ion tracks in diamond-like carbon films. Journal of Applied Physics, 94(3):1959-1964, 2003.

[29] D. Schwen, C. Ronning, and H. Hofsäss. Field emission studies on swift heavy ion irradiated tetrahedral amorphous carbon. Diamond and Related Materials, 13(4-8):1032 - 1036, 2004. 14th European Conference on Diamond, DiamondLike Materials, Carbon Nanotubes, Nitrides and Silicon Carbide.

[30] Hans-Gregor Gehrke, Anne-Katrin Nix, Hans Hofsäss, Johann Krauser, Christina Trautmann, and Alois Weidinger. Self-aligned nanostructures created by swift heavy ion irradiation. Journal of Applied Physics, 107(9):094305, 2010 .

[31] J. Krauser, H.-G. Gehrke, H. Hofsäss, C. Trautmann, and A. Weidinger. Conductive tracks of 30-mev c60 clusters in doped and undoped tetrahedral amorphous carbon. Nuclear Instruments and Methods in Physics Research Section B: Beam Interactions with Materials and Atoms, (0):-, 2013.

[32] A. Nix. Elektrische Eigenschaften von graphitischen Ionenspuren in diamantartigen Kohlenstoffschichten. Master's thesis, Georg-August-Universität Göttingen, 2005.

[33] A. K. Nix, D. Schwen, C. Ronning, J. Krauser, C. Trautmann, and H. Hofsass. Electronic properties of graphite-like ion tracks in insulating tetrahedral amorphous carbon. Reviews On Advanced Materials Science, 15(3):192-197, November 2007.

[34] H. Hofsäss, H. Binder, T. Klumpp, and E. Recknagel. Doping and growth of diamond-like carbon films by ion beam deposition. Diamond and Related Materials, 3(1-2):137 - 142, 1994.

[35] C. Ronning, U. Griesmeier, M. Gross, H.C. Hofsäss, R.G. Downing, and G.P. Lamaze. Conduction processes in boron- and nitrogen-doped diamond-like carbon films prepared by mass-separated ion beam deposition. Diamond and Related Materials, 4(5-6):666 - 672, 1995. ¡ce:title¿Diamond Films '94i/ce:title¿.

[36] J. Krauser, A. K. Nix, H. G. Gehrke, H. Hofsaess, C. Trautmann, and A. Weidinger. Conductivity enhancement of ion tracks in tetrahedral amorphous carbon by doping with N, B, Cu and Fe. Nuclear Instruments and Methods in Physics Research Section B: Beam Interactions with Materials and Atoms, 
272:280-283, FEB 1 2012. 17th International Conference on Ion Beam Modification of Materials (IBMM), Montreal, CANADA, AUG 22-27, 2010.

[37] J. Robertson. Diamond-like amorphous carbon. Materials Science and Engineering: R: Reports, 37(4-6):129 - 281, 2002.

[38] S.R.P. Silva. Properties of Amorphous Carbon. EMIS Datareviews Series. Inspec/Iee, 2003.

[39] C. D. Clark, P. J. Dean, and P. V. Harris. Intrinsic Edge Absorption in Diamond. Royal Society of London Proceedings Series A, 277:312-329, February 1964 .

[40] JOHN C. ANGUS and CLIFF C. HAYMAN. Low-pressure, metastable growth of diamond and "diamondlike" phases. Science, 241(4868):913-921, 1988.

[41] S. Dresselhaus, G. Dresselhaus, and P.C. Eklund. Science of Fullerenes and Carbon Nanotubes: Their Properties and Applications. Elsevier Science, 1996.

[42] Marc Monthioux and Vladimir L. Kuznetsov. Who should be given the credit for the discovery of carbon nanotubes? Carbon, 44(9):1621 - 1623, 2006.

[43] H. W. Kroto, J. R. Heath, S. C. O'Brien, R. F. Curl, and R. E. Smalley. C60: Buckminsterfullerene. Nature, 318(6042):162-163, November 1985.

[44] A. K. Geim and K. S. Novoselov. The rise of graphene. Nat Mater, 6(3):183191, March 2007.

[45] Li-Feng Wang and Quan-Shui Zheng. Extreme anisotropy of graphite and single-walled carbon nanotube bundles. Applied Physics Letters, 90(15):153113, 2007.

[46] L.W. Wang, Z. Tamainot-Telto, S.J. Metcalf, R.E. Critoph, and R.Z. Wang. Anisotropic thermal conductivity and permeability of compacted expanded natural graphite. Applied Thermal Engineering, 30(13):1805 - 1811, 2010.

[47] Y. Lifshitz, S. R. Kasi, J. W. Rabalais, and W. Eckstein. Subplantation model for film growth from hyperthermal species. Phys. Rev. B, 41:10468-10480, May 1990.

[48] James F. Ziegler, M.D. Ziegler, and J.P. Biersack. Srim - the stopping and range of ions in matter (2010). Nuclear Instruments and Methods in Physics Research Section B: Beam Interactions with Materials and Atoms, 268(11-12):1818 - 1823, 2010. 19th International Conference on Ion Beam Analysis. 
[49] P. Neumaier, A. Bergmaier, W. Eckstein, R. Fischer, H. Hofsäss, H. U. Jäger, H. Kröger, C. Ronning, and G. Dollinger. Bimodal range distributions of lowenergy carbon ions in tetrahedral amorphous carbon. EPL (Europhysics Letters), 90(4):46002, 2010.

[50] H. Hofsäss, H. Feldermann, R. Merk, M. Sebastian, and C. Ronning. Cylindrical spike model for the formation of diamondlike thin films by ion deposition. Applied Physics A, 66:153-181, 1998.

[51] C. Ronning. Ion-beam synthesis and growth mechanism of diamond-like materials. Applied Physics A, 77:39-50, 2003.

[52] Michael Nastasi. Ion-solid interactions : fundamentals and applications. Cambridge University Press, Cambridge New York, 1996.

[53] M. Wilde, M. Matsumoto, K. Fukutani, and T. Aruga. Depth-resolved analysis of subsurface hydrogen absorbed by pd. Surface Science, 482-485, Part 1(0):346 $-352,2001$.

[54] J.F. Ziegler. The Stopping and Range of Ions in Matter. Elsevier Science \& Technology Books, 1985.

[55] Stopping Powers and Ranges for Protons and Alpha Particles, volume ICRU Report 49. International Commission on Radiation Units and Measurements, Bethesda, MD, 1993.

[56] N. Bohr. Velocity-range relation for fission fragments. Phys. Rev., 59:270-275, Feb 1941.

[57] H. D. Betz and K. Marton. Atomic Physics Accelerators, volume 17 of Methods in Experimental Physics. Elsevier Science, 1980.

[58] M. Toulemonde. Irradiation by swift heavy ions: Influence of the nonequilibrium projectile charge state for near surface experiments. Nuclear Instruments and Methods in Physics Research Section B: Beam Interactions with Materials and Atoms, 250(1-2):263 - 268, 2006. Proceedings of the 13th International Conference on Radiation Effects in Insulators (REI-2005).

[59] P. L. Grande and G. Schiwietz. Impact-parameter dependence of the electronic energy loss of fast ions. Phys. Rev. A, 58:3796-3801, Nov 1998.

[60] P.L. Grande and G. Schiwietz. Convolution approximation for the energy loss, ionization probability and straggling of fast ions. Nuclear Instruments and Methods in Physics Research Section B: Beam Interactions with Materials and Atoms, 267(6):859 - 865, 2009. Proceedings of the Seventh International Symposium on Swift Heavy Ions in Matter. 
[61] Mohan Singh, Lakhwant Singh, and Navjeet Kaur. Statistical analysis of stopping power of polymers for heavy ions. Materials Physics and Mechanics, 11:95-104, 2011.

[62] E. Baron and C. Ricaud. Beam foil interaction studies for the future stripper of ganil. Conf.Proc., C880607:839, 1988.

[63] O. Herre, W. Wesch, E. Wendler, P. I. Gaiduk, F. F. Komarov, S. Klaumünzer, and P. Meier. Formation of discontinuous tracks in single-crystalline inp by 250-mev xe-ion irradiation. Phys. Rev. B, 58:4832-4837, Aug 1998.

[64] P. Kluth, C. S. Schnohr, O. H. Pakarinen, F. Djurabekova, D. J. Sprouster, R. Giulian, M. C. Ridgway, A. P. Byrne, C. Trautmann, D. J. Cookson, K. Nordlund, and M. Toulemonde. Fine structure in swift heavy ion tracks in amorphous $\mathrm{sio}_{2}$. Phys. Rev. Lett., 101:175503, Oct 2008.

[65] O. Herre, W. Wesch, E. Wendler, P. I. Gaiduk, F. F. Komarov, S. Klaumünzer, and P. Meier. Formation of discontinuous tracks in single-crystalline inp by 250-mev xe-ion irradiation. Phys. Rev. B, 58:4832-4837, Aug 1998.

[66] D. Lesueur and A. Dunlop. Damage creation via electronic excitations in metallic targets part ii : A theoretical model. Radiation Effects and Defects in Solids, 126(1-4):163-172, 1993.

[67] A. Dunlop and D. Lesueur. Damage creation via electronic excitations in metallic targets part i: Experimental results. Radiation Effects and Defects in Solids, 126(1-4):123-128, 1993.

[68] Fr. Dessauer. Über einige Wirkungen von Strahlen. I. Zeitschrift für Physik, $12: 38-47,1923$.

[69] F Seitz and JS Koehler. Displacement of atoms during irradiation. Solid State Physcis-Advances in Research and Applications, 2:305-448, 1956.

[70] I.M. Lifshits, M.I. Kaganov, and L.V. Tanatarov. On the theory of radiationinduced changes in metals. Journal of Nuclear Energy. Part A. Reactor Science, 12(1-2):69-78, 1960 .

[71] M. Beuve, N. Stolterfoht, M. Toulemonde, C. Trautmann, and Herbert M. Urbassek. Influence of the spatial and temporal structure of the depositedenergy distribution in swift-ion-induced sputtering. Phys. Rev. B, 68:125423, Sep 2003.

[72] E.M. Bringa. Molecular dynamics simulations of coulomb explosion. Nuclear Instruments and Methods in Physics Research Section B: Beam Interactions 
with Materials and Atoms, 209(0):1 - 8, 2003. Fifth International Symposium on Swift Heavy Ions in Matter.

[73] M. Toulemonde, Ch. Dufour, A. Meftah, and E. Paumier. Transient thermal processes in heavy ion irradiation of crystalline inorganic insulators. Nuclear Instruments and Methods in Physics Research Section B: Beam Interactions with Materials and Atoms, 166-167(0):903 - 912, 2000.

[74] E. Dartyge and P. Sigmund. Tracks of heavy ions in muscovite mica: Analysis of the rate of production of radiation defects. Phys. Rev. B, 32:5429-5431, Oct 1985.

[75] A. Meftah, M. Hage-Ali, J. P. Stoquert, F. Studer, and M. Toulemonde. Electronic stopping power threshold of damage creation in yttrium iron garnet. Radiation Effects and Defects in Solids, 126(1-4):251-254, 1993.

[76] J. Jensen, A. Dunlop, S. Della-Negra, and M. Toulemonde. A comparison between tracks created by high energy mono-atomic and cluster ions in y3fe5o12. Nuclear Instruments and Methods in Physics Research Section B: Beam Interactions with Materials and Atoms, 146(1-4):412 - 419, 1998.

[77] P. I. Gaiduk, A. Nylandsted Larsen, J. Lundsgaard Hansen, C. Trautmann, and M. Toulemonde. Discontinuous tracks in relaxed si[sub 0.5]ge[sub 0.5] alloy layers: A velocity effect. Applied Physics Letters, 83(9):1746-1748, 2003.

[78] M.E. Toimil Molares, J. Brötz, V. Buschmann, D. Dobrev, R. Neumann, R. Scholz, I.U. Schuchert, C. Trautmann, and J. Vetter. Etched heavy ion tracks in polycarbonate as template for copper nanowires. Nuclear Instruments and Methods in Physics Research Section B: Beam Interactions with Materials and Atoms, 185(1-4):192 - 197, 2001.

[79] Katrin Große, editor. GSI Scientific Report 2011. GSI Helmholtzzentrum für Schwerionenforschung GmbH, Planckstr. 1, 64291 Darmstadt, Germany, May 2012 .

[80] R. Waiblinger, C. Sommerhalter, B. Pietzak, J. Krauser, B. Mertesacker, M. C. Lux-Steiner, S. Klaumunzer, A. Weidinger, C. Ronning, and H. Hofsass. Electrically conducting ion tracks in diamond-like carbon films for field emission. Applied Physics A-materials Science \& Processing, 69(2):239-240, August 1999.

[81] A.C. Ferrari, S.E. Rodil, J. Robertson, and W.I. Milne. Is stress necessary to stabilise sp3 bonding in diamond-like carbon? Diamond and Related Materials, 11(3-6):994 - 999, 2002. 12th European Conference on Diamond, DiamondLike Materials, Carbon Nanotubes, Nitrides \& Silicon Carbide. 
[82] D. S. Grierson, A. V. Sumant, A. R. Konicek, T. A. Friedmann, J. P. Sullivan, and R. W. Carpick. Thermal stability and rehybridization of carbon bonding in tetrahedral amorphous carbon. Journal of Applied Physics, 107(3):033523, 2010 .

[83] Andrea Carlo Ferrari and John Robertson. Raman spectroscopy of amorphous, nanostructured, diamond-like carbon, and nanodiamond. Philosophical Transactions of the Royal Society of London. Series A:Mathematical, Physical and Engineering Sciences, 362(1824):2477-2512, 2004.

[84] G. Schiwietz, G. Xiao, P. L. Grande, E. Luderer, R. Pazirandeh, and U. Stettner. Determination of the electron temperature in the thermal spike of amorphous carbon. EPL (Europhysics Letters), 47(3):384, 1999.

[85] A. Dunlop, G. Jaskierowicz, J. Jensen, and S. Della-Negra. Track separation due to dissociation of mev c60 inside a solid. Nuclear Instruments and Methods in Physics Research Section B: Beam Interactions with Materials and Atoms, 132(1):93 - 108, 1997.

[86] J.-M. Costantini, J.-P. Salvetat, F. Couvreur, and S. Bouffard. Carbonization of polyimide by swift heavy ion irradiations: Effects of stopping power and velocity. Nuclear Instruments and Methods in Physics Research Section B: Beam Interactions with Materials and Atoms, 234(4):458 - 466, 2005.

[87] T. Seidl, A. Plotnikov, E. Mustafin, R. Lopez, D. Severin, E. Floch, C. Trautmann, A. Golubev, A. Smolyakov, D. Tommasini, and W. Ensinger. Influence of swift heavy ion beams and protons on the dielectric strength of polyimide. Polymer Degradation and Stability, 97(11):2396 - 2402, 2012.

[88] A. Tripathi, Amit Kumar, D. Kabiraj, S.A. Khan, V. Baranwal, and D.K. Avasthi. Shi induced conducting tracks formation in c60. Nuclear Instruments and Methods in Physics Research Section B: Beam Interactions with Materials and Atoms, 244(1):15 - 18, 2006. Proceedings of the Indo German Workshop on Synthesis and Modifications of Nano-Structured Materials by Energetic Ion Beams.

[89] Ulrich A. Glasmacher, Maik Lang, Hans Keppler, Falko Langenhorst, Reinhard Neumann, Dieter Schardt, Christina Trautmann, and Günther A. Wagner. Phase transitions in solids stimulated by simultaneous exposure to high pressure and relativistic heavy ions. Phys. Rev. Lett., 96:195701, May 2006.

[90] Paul Ehrhardt. Modifikation der elektrischen Eigenschaften von Vanadiumdioxid durch Ionenbestrahlung. Master's thesis, Georg-August Universität, Göttingen, 2013. 
[91] H. Hofsäss, P. Ehrhardt, H.-G. Gehrke, M. Brtzmann, U. Vetter, K. Zhang, J. Krauser, C. Trautmann, C. Ko, and S. Ramanathan. Tuning the conductivity of vanadium dioxide films on silicon by swift heavy ion irradiation. AIP Advances, 1(3):032168, 2011.

[92] Masanori Koshimizu, Hirotaka Tsukahara, and Keisuke Asai. Formation of metastable conductive nanowire in a thiospinel compound $\mathrm{CuIr}_{2} \mathrm{~S}_{4}$ induced by ion irradiation. Nuclear Instruments and Methods in Physics Research Section B: Beam Interactions with Materials and Atoms, 267(7):1125 - 1128, 2009.

[93] Ohm G. S. Bestimmung des Gesetzes, nach welchem Metalle die Conaktelektricität leiten, nebst einem Entwurfe zu einer Theorie des Voltarischen Apparates und des Schweiggerschen Multiplicators. Journal für Chemie und Physik, 16:137-166, 1826.

[94] J. J. Thomson. Cathode Rays. Philosophical Magazine, 44(1):293-316, 1897.

[95] P. Drude. Zur Elektronentheorie der Metalle. Annalen der Physik, 306(3):566$613,1900$.

[96] Charles Kittel. Introduction to solid state physics. Wiley, Hoboken, NJ, 2005.

[97] Neil Ashcroft. Solid state physics. Holt, Rinehart and Winston, New York, 1976.

[98] H Ibach and H Lüth. Solid-state physics an introduction to principles of materials science. Springer, Berlin New York, 2009.

[99] P. Debye. Zur Theorie der spezifischen Wärmen. Annalen der Physik, 344(14):789-839, 1912.

[100] A. Einstein. Die Plancksche Theorie der Strahlung und die Theorie der spezifischen Wärme. Annalen der Physik, 327(1):180-190, 1906.

[101] S.M. Sze and K.K. Ng. Physics of Semiconductor Devices. Wiley, 2006.

[102] J. Orloff. Handbook of Charged Particle Optics, Second Edition. Taylor \& Francis, 2008.

[103] M. Brötzmann. Electrical characterization of Metal - Amorphous Semiconductor - Semiconductor diodes - a general conduction model. PhD thesis, GeorgAugust-Universität Göttingen, 2013.

[104] F. Schwabl. Quantenmechanik (QM I). Springer-Lehrbuch. Springer London, Limited, 2007. 
[105] T. Fließbach. Quantenmechanik: Lehrbuch zur Theoretischen Physik III. Fließbach, Torsten: Lehrbuch zur theoretischen Physik. Spektrum Akademischer Verlag, 2008.

[106] J G Simmons. Conduction in thin dielectric films. Journal of Physics D: Applied Physics, 4(5):613, 1971.

[107] J. Frenkel. On pre-breakdown phenomena in insulators and electronic semiconductors. Phys. Rev., 54:647-648, Oct 1938.

[108] John G. Simmons. Poole-frenkel effect and schottky effect in metal-insulatormetal systems. Phys. Rev., 155:657-660, Mar 1967.

[109] O Bleibaum, H Bottger, and VV Bryksin. Impact of the density of states on the dynamical hopping conductivity. Phys. Rev. B, 66(10), SEP 12002.

[110] M Grunewald, P Thomas, and D Wurtz. Simple calculation of the hopping conductivity for an exponential band tail. Physica status solidi b-basic research, 94(1):K1-K4, 1979.

[111] M Grunewald and P Thomas. Hopping model for activated charge transport in amorphous silicon. Physica status solidi b-basic research, 94(1):125-133, 1979.

[112] M.M El-Samanoudy. Modified poole-frenkel mechanisms in ge25bixsb15-xs60 thin films. Applied Surface Science, 207(1-4):219 - 226, 2003.

[113] R.B. Hall. The poole-frenkel effect. Thin Solid Films, 8(4):263 - 271, 1971.

[114] Robert M. Hill. Poole-frenkel conduction in amorphous solids. Philosophical Magazine, 23(181):59-86, 1971.

[115] A.K. Jonscher. Electronic properties of amorphous dielectric films. Thin Solid Films, 1(3):213 - 234, 1967.

[116] J. L. Hartke. The three-dimensional poole-frenkel effect. Journal of Applied Physics, 39(10):4871-4873, 1968.

[117] N.F. Mott. Conduction in glasses containing transition metal ions. Journal of Non-Crystalline Solids, 1(1):1 - 17, 1968.

[118] J. J. van Hapert. Hopping Conduction and Chemical Structure a study on Silicon Suboxides. PhD thesis, Universiteit Utrecht, Faculteit Natuur- en Sterrenkunde, 2002.

[119] N Apsley and HP Hughes. Temperature-dependence and field-dependence of hopping conduction in disordered systems. Philosophical Magazine, 30(5):963972, 1974. 
[120] N Apsley and HP Hughes. Temperature-dependence and field-dependence of hopping conduction in disordered systems .2. Philosophical Magazine, 31(6):1327-1339, 1975.

[121] D Bourbie. Nonohmic hopping conductivity in disordered-systems. Philosophical magazine b-physics of condensed matter statistical mechanics electronic optical and magnetic properties, 68(1):47-54, JUL 1993.

[122] D Bourbie. Theory of hopping transport in disordered systems. Philosophical magazine b-physics of condensed matter statistical mechanics electronic optical and magnetic properties, 73(2):201-212, FEB 1996.

[123] G. E. Pike and C. H. Seager. Percolation and conductivity - computer study .1. Physical Review B, 10(4):1421-1434, 1974.

[124] C. H. Seager and G. E. Pike. Percolation and conductivity - computer study .2. Physical Review B, 10(4):1435-1446, 1974.

[125] N.F. Mott and E.A. Davis. Electronic Processes in Non-Crystalline Materials. Oxford Classic Texts in the Physical Sciences. Clarendon Press Oxford, 1979.

[126] Allen Miller and Elihu Abrahams. Impurity conduction at low concentrations. Phys. Rev., 120:745-755, Nov 1960.

[127] Kazuo Morigaki. Physics Amorphous Semiconductors. Imperial College Press, 1999.

[128] Charge transport in disordered solids with applications in electronics. Wiley, Chichester, England Hoboken, NJ, 2006.

[129] J. M. Marshall. Computer simulation studies of low-temperature hopping in spatially and energetically disordered systems. Journal of optoelectronics and advanced materials, 9:84-91, 2007.

[130] E. M. Hamilton. Variable range hopping in a non-uniform density of states. Philosophical Magazine, 26(4):1043-1045, 1972.

[131] M. Pollak and G. E. Pike. ac conductivity of glasses. Phys. Rev. Lett., 28:14491451, May 1972.

[132] M Pollak and I Riess. A percolation treatment of high-field hopping transport. Journal of Physics C: Solid State Physics, 9(12):2339, 1976.

[133] SL Maeng, S Uchikoga, FJ Clough, A Tagliaferro, AJ Flewitt, J Robertson, and WI Milne. A carbon based bottom gate thin film transistor. Diamond and Related Materials, 9(3-6):805-810, APR-MAY 2000. 10th European Conference 
on Diamond, Diamond-Like Materials, Nitrides and Silicon Carbide (Diamond 1999).

[134] N.L. Rupesinghe, R.J. Cole, M. Chhowalla, G.A.J. Amaratunga, and P. Weightman. Tetrahedral amorphous carbon-silicon heterojunction band energy offsets. Diamond and Related Materials, 9(3-6):1148 - 1153, 2000.

[135] P. Stumm, D. A. Drabold, and P. A. Fedders. Defects, doping, and conduction mechanisms in nitrogen-doped tetrahedral amorphous carbon. Journal of Applied Physics, 81(3):1289-1295, 1997.

[136] C. Arena, B. Kleinsorge, J. Robertson, W. I. Milne, and M. E. Welland. Electronic properties of tetrahedral amorphous carbon investigated by scanning tunneling microscopy. Journal of Applied Physics, 85(3):1609-1615, 1999.

[137] J. Robertson. Defects in diamond-like carbon. physica status solidi (a), 186(2):177-185, 2001.

[138] Y. Miyajima, S. J. Henley, G. Adamopoulos, V. Stolojan, E. Garcia-Caurel, B. Drévillon, J. M. Shannon, and S. R. P. Silva. Pulsed laser deposited tetrahedral amorphous carbon with high $\mathrm{sp}^{3}$ fractions and low optical bandgaps. Journal of Applied Physics, 105(7):073521, 2009.

[139] Godet C. Physics of bandtail hopping in disordered carbons. Diamond and Related Materials, 12(2):159-165, 2003.

[140] C.W Chen and J Robertson. Nature of disorder and localization in amorphous carbon. Journal of Non-Crystalline Solids, 227-230, Part 1(0):602 - 606, 1998.

[141] J. Robertson. Gap states in diamond-like amorphous carbon. Philosophical Magazine Part B, 76(3):335-350, 1997.

[142] S. Koizumi, M. Kamo, Y. Sato, H. Ozaki, and T. Inuzuka. Growth and characterization of phosphorous doped 111 homoepitaxial diamond thin films. Applied Physics Letters, 71(8):1065-1067, 1997.

[143] N.F. Mott. Electrons in disordered structures. Advances in Physics, 16(61):49$144,1967$.

[144] W. E. Spear, P. G. Le Comber, S. Kinmond, and M. H. Brodsky. Amorphous silicon p-n junction. Applied Physics Letters, 28(2):105-107, 1976.

[145] P.K. Sitch, Th. Köhler, G. Jungnickel, D. Porezag, and Th. Frauenheim. A theoretical study of boron and nitrogen doping in tetrahedral amorphous carbon. Solid State Communications, 100(8):549 - 553, 1996. 
[146] P. W. Anderson. Model for the electronic structure of amorphous semiconductors. Phys. Rev. Lett., 34:953-955, Apr 1975.

[147] R.A Street and D.K Biegelsen. Luminescence and esr studies of defects in hydrogenated amorphous silicon. Solid State Communications, 33(12):1159 1162, 1980.

[148] John Robertson. Electronic and atomic structure of diamond-like carbon. Semiconductor Science and Technology, 18(3):S12, 2003.

[149] F. Weich, J. Widany, and Th. Frauenheim. Paracyanogenlike structures in high-density amorphous carbon nitride. Phys. Rev. Lett., 78:3326-3329, Apr 1997.

[150] J Robertson and CA Davis. Nitrogen doping of tetrahedral amorphous-carbon. Diamond and Related Materials, 4(4):441-444, APR 15 1995. 5th European Conference on Diamond, Diamond-Like and Related Materials (Diamond Films $94)$.

[151] B Kleinsorge, A Ilie, M Chhowalla, W Fukarek, WI Milne, and J Robertson. Electrical and optical properties of boronated tetrahedrally bonded amorphous carbon (ta-C : B). Diamond and Related Materials, 7(2-5):472-476, FEB 1998. 8th European Conference on Diamond, Diamond-like and Related Materials/4th International Conference on the Applications of Diamond Films and Related Materials, EDINBURGH, SCOTLAND, AUG 03-08, 1997.

[152] T. B. Massalski. Binary alloy phase diagrams. ASM International, Materials Park, Ohio, 1990.

[153] I. Gerhards, C. Ronning, H. Hofsäss, M. Seibt, and H. Gibhardt. Ion beam synthesis of diamond-like carbon thin films containing copper nanocrystals. Journal of Applied Physics, 93(2):1203-1207, 2003.

[154] Sukang Bae, Hyeongkeun Kim, Youngbin Lee, Xiangfan Xu, Jae-Sung Park, Yi Zheng, Jayakumar Balakrishnan, Tian Lei, Hye Ri Kim, Young Il Song, Young-Jin Kim, Kwang S. Kim, Barbaros Ozyilmaz, Jong-Hyun Ahn, Byung Hee Hong, and Sumio Iijima. Roll-to-roll production of 30-inch graphene films for transparent electrodes. Nat Nano, 5(8):574-578, August 2010.

[155] Xuesong Li, Weiwei Cai, Jinho An, Seyoung Kim, Junghyo Nah, Dongxing Yang, Richard Piner, Aruna Velamakanni, Inhwa Jung, Emanuel Tutuc, Sanjay K. Banerjee, Luigi Colombo, and Rodney S. Ruoff. Large-area synthesis of high-quality and uniform graphene films on copper foils. Science, 324(5932):1312-1314, 2009. 
[156] Inga Gerhards. Self-Organized Formation of Metal-Carbon Nanostructures by Hyperthermal Ion Deposition. PhD thesis, Georg-August Universität, Göttingen, 2006.

[157] Hayo Zutz. Growth and Characterization of Carbon-Metal-NanocompositeThin-Films and Self-Organized Layer Growth. PhD thesis, Georg-AugustUniversität zu Göttingen, 2009.

[158] I. Gerhards, H. Stillrich, C. Ronning, H. Hofsäss, and M. Seibt. Self-organized nanoscale multilayer growth in hyperthermal ion deposition. Phys. Rev. B, 70:245418, Dec 2004.

[159] David Williams and C. Barry Carter. Transmission electron microscopy a textbook for materials science. Springer, New York, 2009.

[160] A. Einstein. Über einen die Erzeugung und Verwandlung des Lichtes betreffenden heuristischen Gesichtspunkt. Annalen der Physik, 322(6):132-148, 1905.

[161] Andrew Zangwill. Physics at surfaces. Cambridge University Press, Cambridge Cambridgeshire New York, 1988.

[162] John C. Fuggle and Nils Mårtensson. Core-level binding energies in metals. Journal of Electron Spectroscopy and Related Phenomena, 21(3):275 - 281, 1980.

[163] NIST X-ray Photoelectron Spectroscopy Database. National Institute of Standards and Technology, Gaithersburg, 4.1 edition, 2012.

[164] C Ronning, H Feldermann, R Merk, H Hofsass, P Reinke, and JU Thiele. Carbon nitride deposited using energetic species: A review on XPS studies. Phys. Rev. B, 58(4):2207-2215, JUL 151998.

[165] G. Binnig, C. F. Quate, and Ch. Gerber. Atomic force microscope. Phys. Rev. Lett., 56:930-933, Mar 1986.

[166] P Mérel, M Tabbal, M Chaker, S Moisa, and J Margot. Direct evaluation of the sp3 content in diamond-like-carbon films by xps. Applied Surface Science, 136(1-2):105 - 110, 1998.

[167] S. D. Berger, D. R. McKenzie, and P. J. Martin. Eels analysis of vacuum arcdeposited diamond-like films. Philosophical Magazine Letters, 57(6):285-290, 1988 .

[168] P Reinke, M.G Garnier, and P Oelhafen. In situ photoelectron spectroscopy analysis of tetrahedral amorphous carbon films. Journal of Electron Spectroscopy and Related Phenomena, 136(3):239 - 245, 2004. 
[169] J.J. Yeh and I. Lindau. Atomic subshell photoionization cross sections and asymmetry parameters: $1 \leqslant \mathrm{Z} \leqslant 103$. Atomic Data and Nuclear Data Tables, 32(1):1 - 155, 1985.

[170] Marc Broetzmann, Hans-Gregor Gehrke, Ulrich Vetter, and Hans Hofsaess. Modeling the diode characteristics of boron nitride/silicon carbide heterojunctions. Applied Physics Letters, 97(10), SEP 62010.

[171] M. Broetzmann, U. Vetter, and H. Hofsaess. BN/ZnO heterojunction diodes with apparently giant ideality factors. Journal of Applied Physics, 106(6), SEP 152009.

[172] Python2.7. http://www.python.org/

[173] Eric Jones, Travis Oliphant, Pearu Peterson, et al. SciPy: Open source scientific tools for Python, $2001-2013$.

[174] C Riedel and R Spohr. Correcting overlapping counts in dose calibration at high event-densities. Nuclear tracks and radiation measurements, 5(3):265-270, 1981.

[175] Neeraj Dwivedi, Sushil Kumar, J. D. Carey, Hitendra K. Malik, and Govind. Photoconductivity and characterization of nitrogen incorporated hydrogenated amorphous carbon thin films. Journal of Applied Physics, 112(11):113706, 2012.

[176] EB Maiken and P Taborek. Amorphous carbon films deposited from carbon ions extracted from a discharge in fullerene vapor. Journal of Applied Physics, 87(9, Part 1):4223-4229, MAY 12000.

[177] David Perello, Woojong Yu, Dong Jae Bae, Seung Jin Chae, Moon J. Kim, Young Hee Lee, and Minhee Yun. Pool-frenkel emission and hopping conduction in semiconducting carbon nanotube transistor. pages 739907-739907-6, 2009.

[178] David J. Perello, Woo Jong Yu, Dong Jae Bae, Seung Jin Chae, M. J. Kim, Young Hee Lee, and Minhee Yun. Analysis of hopping conduction in semiconducting and metallic carbon nanotube devices. Journal of Applied Physics, 105(12):124309, 2009.

[179] S. M. Sze. Current transport and maximum dielectric strength of silicon nitride films. Journal of Applied Physics, 38(7):2951-2956, 1967.

[180] L Sullivan and H C Card. Conduction in amorphous thin films of silicon nitride under non-uniform electric fields. Journal of Physics D: Applied Physics, 7(11):1531, 1974. 
[181] J. J. O'Dwyer. Current-voltage characteristics of dielectric films. Journal of Applied Physics, 37(2):599-601, 1966.

[182] Klaus H. Ploog. Single charge tunneling, coulomb blockade phenomena in nanostructures. Advanced Materials, 5(3):227-227, 1993.

[183] S. J. Shin, J. J. Lee, H. J. Kang, J. B. Choi, S.-R. Eric Yang, Y. Takahashi, and D. G. Hasko. Room-temperature charge stability modulated by quantum effects in a nanoscale silicon island. Nano Letters, 11(4):1591-1597, 2011.

[184] Z.A.K. Durrani. Single-Electron Devices and Circuits in Silicon. Imperial College Press, 2010.

[185] Dieter Bimberg, Marius Grundmann, Nikolai N Ledentsov, et al. Quantum dot heterostructures, volume 471973882. John Wiley Chichester, 1999.

[186] Y.A. Pashkin, Y. Nakamura, and J.S. Tsai. Room-temperature al singleelectron transistor made by electron-beam lithography. Applied Physics Letters, 76(16):2256-2258, 2000.

[187] T Teranishi, M Haga, Y Shiozawa, and M Miyake. Self-organization of au nanoparticles protected by 2, 6-bis (1"-(8-thiooctyl) benzimidazol-2-yl) pyridine. Journal-American chemical society, 122(17):4237-4238, 2000.

[188] M Dorogi, J Gomez, R Osifchin, RP Andres, and R Reifenberger. Roomtemperature coulomb blockade from a self-assembled molecular nanostructure. Physical Review B, 52(12):9071, 1995.

[189] K. I. Bolotin, F. Kuemmeth, A. N. Pasupathy, and D. C. Ralp. Metalnanoparticle single-electron transistors fabricated using electromigration. Applied Physics Letters, 84(16):3154-3156, 2004.

[190] R. P. Andres, S. Datta, M. Dorogi, J. Gomez, J. I. Henderson, D. B. Janes, V. R. Kolagunta, C. P. Kubiak, W. Mahoney, R. F. Osifchin, R. Reifenberger, M. P. Samanta, and W. Tian. Room temperature coulomb blockade and coulomb staircase from self-assembled nanostructures. volume 14, pages 1178-1183. AVS, 1996. 


\section{Curriculum vitae}

\section{Persönliche Daten:}

Name:

Hans-Gregor Gehrke

Geburtstag:

29.06.1983

Geburtsort:

Merseburg

Nationalität:

deutsch

\section{Bildungsweg:}

2002: International Baccalaureate Awty International School, Houston (USA)

2003: $\quad$ Beginn Physikstudium an der Georg-August-Universität, Göttingen

2006: $\quad$ Vordiplom Physik

2009: Diplom Physik

Georg-August-Universität, Göttingen

2009-2012: wissenschaftlicher Mitarbeiter

Georg-August-Universität, Göttingen 


\section{List of publications:}

J. Krauser, A.-K. Nix, H.-G. Gehrke, H. Hofsäss, C. Trautmann, A. Weidinger, F. Wünsch, and J. Bruns. Ion track lithography and graphitic nanowires in diamondlike carbon Journal of vacuum science \& technology B, 26(6):2468, 2008.

A.-K. Nix, H.-G. Gehrke, J. Krauser, C. Trautmann, A. Weidinger, and H. Hofsäss. Track-etched nanopores in spin-coated polycarbonate films applied as sputtering mask Nuclear instruments \& methods in physics research section B - beam interactions with materials and atoms, 267(6):1032, 2009.

H.-G. Gehrke, A.-K. Nix, H. Hofsäss, J. Krauser, C. Trautmann, and A. Weidinger. Self-aligned nanostructures created by swift heavy ion irradiation Journal of applied physics, 107(9):094305, 2010.

M. Brötzmann, H.-G. Gehrke, U. Vetter, and H. Hofsäss. Modeling the diode characteristics of boron nitride/silicon carbide Applied physics letters, 97(10):103505, 2010.

J. Krauser, A.-K. Nix, H.-G. Gehrke, H. Hofsäss, C. Trautmann, and A. Weidinger. Highly conductive ion tracks in tetrahedral amorphous carbon by irradiation with 30 MeV C-60 projectiles New journal of physics, 13:083023, 2011.

H. Hofsäss, P. Ehrhardt, H.-G. Gehrke, M. Brötzmann, U. Vetter, K. Zhang, J. Krauser, C. Trautmann, C. Ko, and S. Ramanathan Tuning the conductivity of vanadium dioxide films on silicon by swift heavy ion irradiation AIP advances, 1(3):032168, 2011.

D. Jürgens, M. Uhrmacher, H.-G. Gehrke, M. Nagl, U. Vetter, C. Brüsewitz, H. Hofsäss, J. Mestnik-Filho, and M. W. Barsoum. Electric field gradients at In-111/Cd111 probe atoms on $A$-sites in 211-MAX phases Journal of physics-condensed matter, 23(50):505501, 2011.

J. Krauser, A.-K. Nix, H.-G. Gehrke, H. Hofsäss, C. Trautmann, and A. Weidinger. Conductivity enhancement of ion tracks in tetrahedral amorphous carbon by doping with $N, B, C u$ and $F e$ Nuclear instruments \& methods in physics research section B, 272:280, 2012.

J. Krauser, H.-G. Gehrke, H. Hofsäss, C. Trautmann, and A. Weidinger. Conductive tracks of 30-MeV C60 clusters in doped and undoped tetrahedral amorphous carbon Nuclear instruments \& methods in physics research section B, (in print) 2013. 


\section{Danksagungen}

Ich möchte mich zuerst bedanken bei:

- Herrn Prof. Dr. Hofsäss für die Ermöglichung meiner Promotion im 2. Institut und die vielen internationalen Konferenzbesuche.

- Herrn Prof. Dr. Jooß für seine Dienste als Koreferent.

- Herrn PD Dr. Grosse-Knetter, Herrn Prof. Dr. Müntzenberg, Herrn Prof. Dr. Krebs und Herrn Prof. Dr. Seibt für die Bereitschaft zur Besetzung der Prüfungskommission.

- Herrn Prof. Dr. Johann Krauser für die tatkräftige Hilfe bei den Anfertigung und Diskussion der AFM Bilder. Danke für die herzliche Zusammenarbeit.

- Frau Dr. Christina Trautmann für die Bestrahlung an der GSI - besonders für spontane parasitic beamtime. Danke für deine nette Unterstützung.

- Herrn Prof. Dr. Marcel Toulemonde für die unkomplizierte Organisation der $\mathrm{Pb}$ Bestrahlung am GANIL - Merci beaucoup!

- Herrn Prof. Dr. Hermann Rothard für die Pb Bestrahlung am Ganil.

- Herrn Dr. Omar Kamalou für hilfreiche Informationen und Daten zur Strahlcharakteristik am GANIL

- Herrn Dr. Marc Brötzmann für hilfreiche Diskussionen und die Anfertigung der TEM Bilder

- Herrn Prof. Dr. Alois Weidinger für die fruchtbaren Diskussionen und das Korrekturlesen meiner Arbeit. Vielen Dank für die Motivation, diese Arbeit endlich niederzuschreiben!

- Herrn Dr. Ulrich Vetter für die Betreuung und Korrektur meiner Arbeit sowie die unzähligen Kleinigkeiten.

- Frau Regina Gehrke für das Korrekturlesen meiner Arbeit.

Ich möchte mich auch bei allen netten Kolleginnen und Kollegen für die schöne Zeit im Institut und an der Universität Göttingen bedanken. Ein herzlicher Dank gilt den Mitarbeitern der Werkstatt - ohne euch funktioniert Experimentalphysik nicht. 
Ich möchte auch allen einen herzlichen Dank aussprechen, die mich persönlich unterstützt haben. Besonders erwähnen möchte ich Daniel Jürgens und Andreas König - Danke für eure Hilfsbereitschaft, Spendierfreudigkeit und die lustigen nicht-physikalischen Mensagespräche. Paul, dir danke ich für deine ruhige und lustige Natur. Dr. Marc Brötzmann, deine Hilfe ging weit über das Messen und Diskutieren hinaus. Wir haben zusammen angefangen zu studieren und werden nun zusammen fertig! Du bist nicht nun mein gutmütiger Bürokollege gewesen, sondern vor allem mein guter Freund geworden - vielen Dank für Alles was du getan hast.

Meinen Eltern und meinen Geschwistern gilt ebenso ein herzliches Dankeschön. Ihr habt mich am längsten betreut und bis hierher gebracht. Ohne euch könnte ich diese Worte jetzt nicht schreiben.

Schließlich gilt mein Dank dir Suji - Du meine Seele, mein Herz! 